QL

415

N3J 66

1915

MOLL 


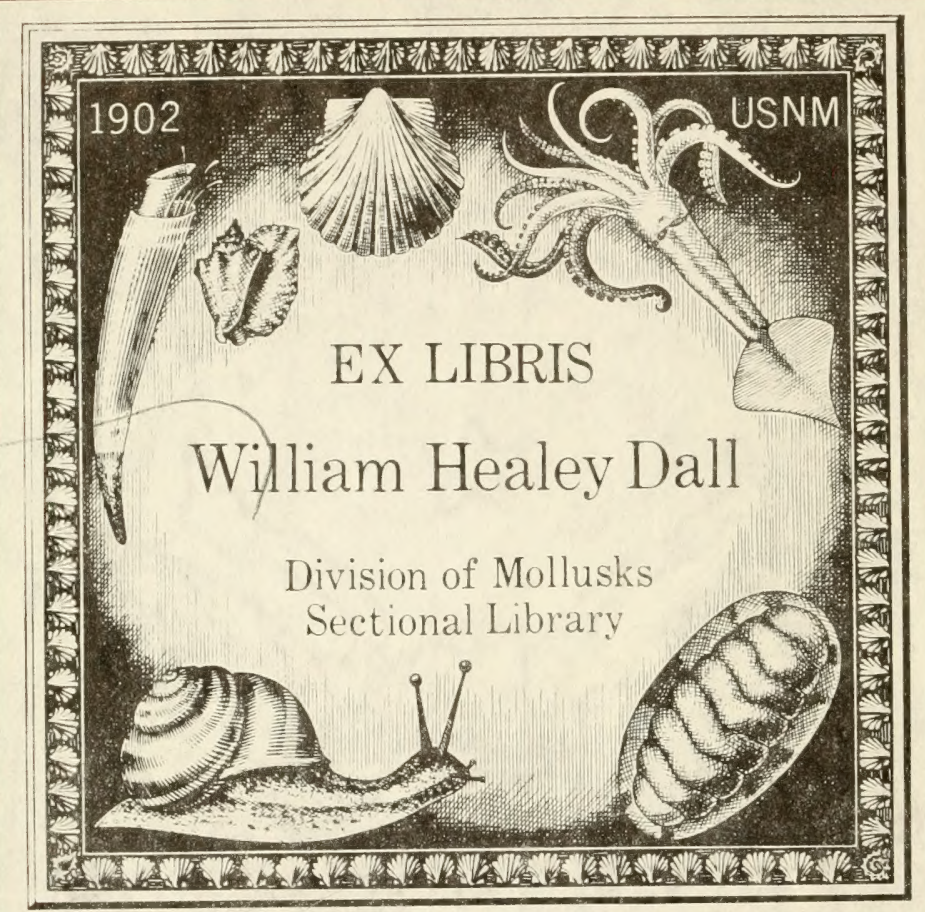




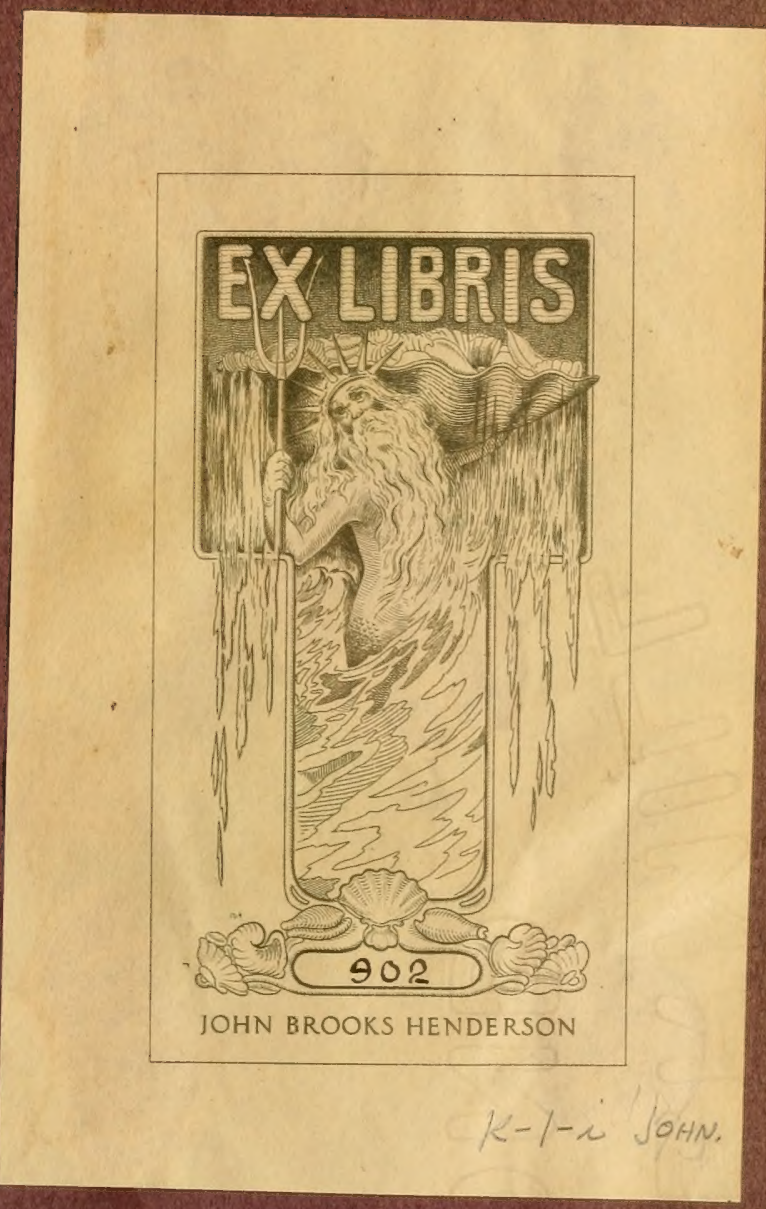





Oectasional fapers

OF THE

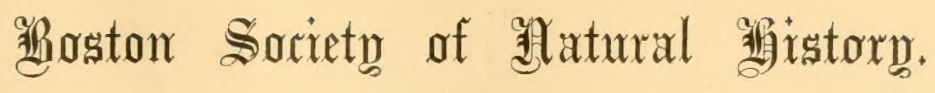

\title{
VII.
}

\author{
Division of Mollusiles \\ Sectional Library
}

\section{FAUNA OF NEW ENGLAND.}

\section{3.}

LIST OF THE MOLLUSCA.

By CHARLES W. JOHNSON.

BOSTON:

PRINTED FOR THE SOCIETY FROM THE

GURDON SALTONSTALL FUND.

December, 1915. 
<smiles>C1CCCC1</smiles>

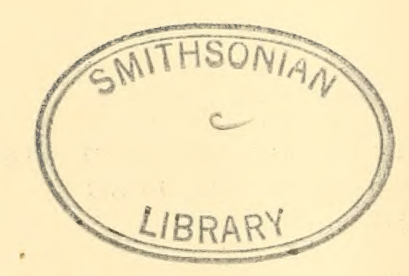




\section{INTRODUCTION.}

THE preparation of a faunal list of New England Mollusca at this time, when nomenclature is so unsettled by the adoption of the genera of Bolten and of other early writers, whose works were either overlooked or ignored by the "old school," is fraught with sad misgivings as one sees many of the names familiar from boyhood swept into the synonymic sea. Though fully believing in the law of priority, I should feel some hesitancy in presenting these names in a faunal list, were it not for the fact that practically all of these changes have been recently published, but so scattered through various journals and papers that their adoption has not yet become general.

The Gould-Binney edition of the Invertebrates of Massachusetts, published in 1870, is still the book on New England Mollusca and will continue to be for some time; therefore in preparing this list all of the names used in that work that have been changed, are given in the synonymy. The second work bearing directly on the fauna is Verrill's Report upon the Invertebrate Animals of Vineyard Sound and Adjacent Waters, published by the United States Commission of Fish and Fisheries in 1873. This was followed by his "Catalogue of Marine Mollusca added to the fauna of the New England coast and the adjacent parts of the Atlantic consisting mostly of deep sea species " published in three parts in the Transactions of the Connecticut Academy, 1882, 1884, and 1885. As the latter papers contained many species found far beyond what can be reasonably considered New England, the necessity of establishing a New England marine faunal area became apparent. A paper on this subject with the accompanying map was published in the Society's Museum and Library Bulletin, No. 7, May, 1908.

In forming this area we should take into consideration the diversity of conditions off our coast, such as the great irregularity in depth, the effect of currents and tides on the temperature of the water, and the character of the sea bottom. These are impor- 
tant factors governing distribution and the area should be large enough to enable one to study the effects of these varied conditions to advantage. To appreciate fully the true gulf or land-locked character of the Gulf of Maine, one should study a chart covering the Gulf, adjacent coast lines, and banks. This shows at once the desirability of adopting the 200-fathom line, as any less depth would give a very irregular and impracticable boundary, there being several places in the Gulf of Maine deeper than 150 fathoms but not exceeding 200 fathoms, while all of the Georges Bank is less than 50 fathoms. The most convenient eastern boundary therefore would be the 66 th meridian which just grazes the eastern edge of the Georges and includes the approach to the Bay of Fundy, the tides of which have such a great influence on the fauna of the Maine coast. On the eastern edge of the Georges at about $40^{\circ} 30^{\prime}$ north latitude the 66 th meridian intersects the 200 -fathom line, which practically skirts the edge of the continental shelf to a point directly south of Montauk Point, Long Island,-- about $40^{\circ}$ north latitude and $72^{\circ}$ west longitude. This gives an area south of the Georges and Cape Cod, sufficient for the study of the influences of the Gulf Stream and counter currents. All of Long Island Sound except the bays and harbors of Long Island is also included in this area.

The land mollusks also present many interesting features in distribution, since New England is the northeastern limit of distribution of many species, such as Polygyra palliata, Polygyra tridentata, Omphalina fuliginosa, Omphalina inornata, etc., species common to the mountains of North Carolina and Tennessee. We can trace these species back and find that their line of dispersal from that region was probably first northward along the western ridges of the Alleghanies into central New York and thence eastward along the valleys of the Mohawk and Hudson Rivers into western New England. The distribution of Helix hortensis, confined to the islands and headlands of the coast, strengthens the theory of botanists and others of a large continuous land area to the eastward in comparatively recent geological times.

Of the species from deep water, only those actually taken within 


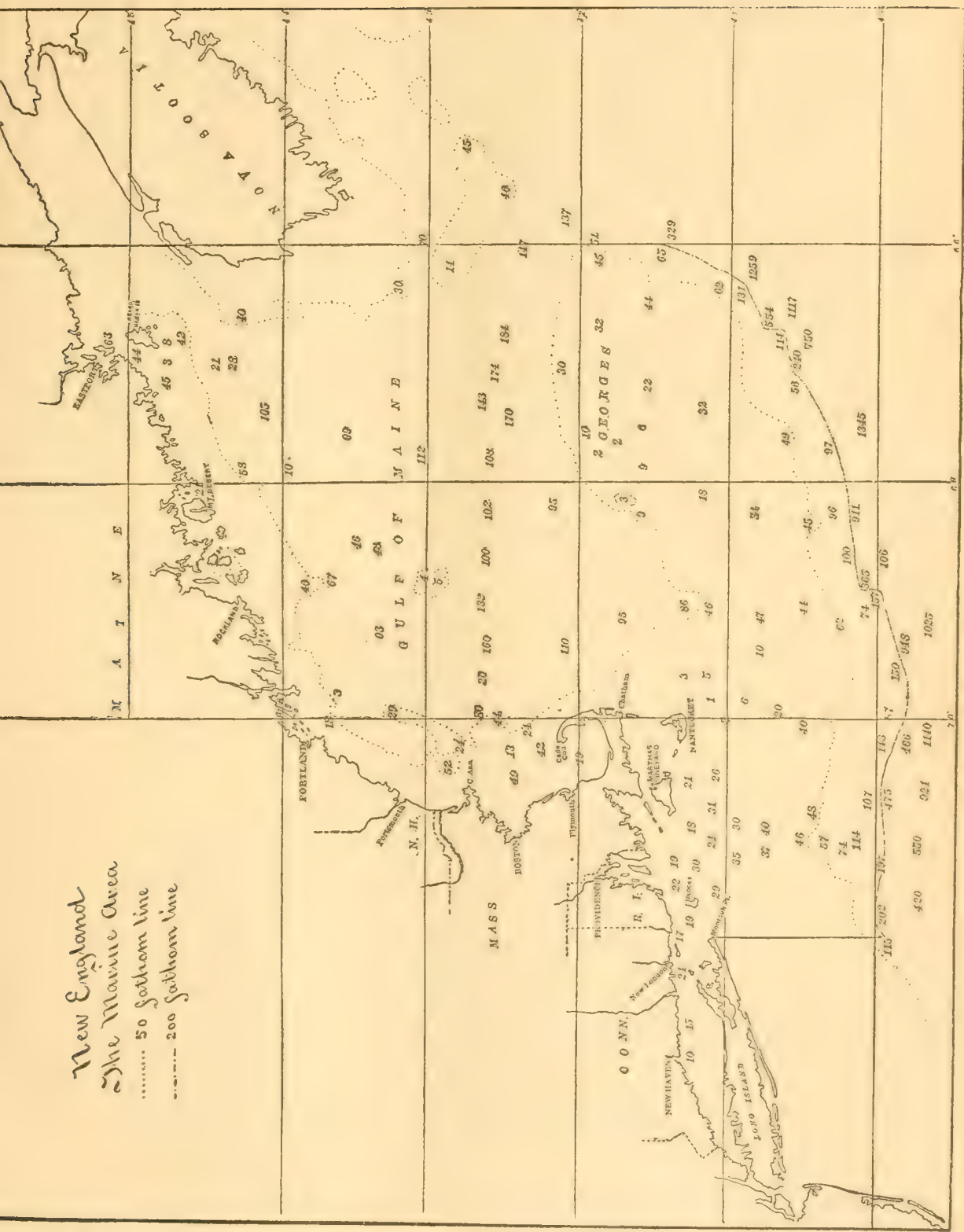


the described area have been included, and as the Society has very few of those found below 50 fathoms, I have been unable to verify a large number of the species recorded. The list contains 738 species and 71 varieties. Those marked with an asterisk are in the collection of the Society and additions to the New England collection are especially desired.

In the work of preparing this list the writer is indebted to Francis N. Balch, Esq., for the list of Nudibranchs, to Dr. Henry B. Bigelow for many of the records of the Pteropods, to Dr. V. Sterki for assistance with the Sphaeridae, and to Messrs. William F. Clapp, Henry W. Winkley, Owen Bryant, Olof O. Nylander, N. W. Lermond, and others for specimens and records of distribution. The various collecting trips made for the Society by Dr. Joseph A. Cushman and the writer have not only added to our knowledge of distribution, but have also greatly enriched the collection. 


\section{MOLLUSCA.}

\section{AMPHINEURA.}

POLYPLACOPHORA.

\section{LEPIDOPLEURIDAE.}

Lepidopleurus alveolus (Lovén).

Pilsbry, Manual Conch., 1892, ser. 1, vol. 14, p. 6, pl. 2, figs. 23-31.

Chiton alveolus (Sars MS.) Lovén, Öfvers. Kongl. Vet.-Akad. Förh., 1846, vol. 3, p. 159. "BoH[us]-Bergen."

Gulf of Maine, 150 fathoms; Georges Bank.

\section{Lepidopleurus cancellatus (Sowerby).}

Pilsbry, Manual Conch., 1892, ser. 1, vol. 14, p. 3, pl. 3, figs. 54-58.

Chiton cancellatus Sowerby, Conch. Illustr., 1839, pt. 167, figs. 104, 105. [Great Britain.]

Leptochiton cancellatus Verrill, Trans. Conn. Acad., 1882, vol. 5, p. 534.

Gulf of Maine, Cashes Ledge, 30 to 40 fathoms (Verrill).

\section{Hanleya hanleyi (Bean).}

Pilsbry, Manual Conch., 1892, ser. 1, vol. 14, p. 17, pl. 3, figs. 71-79.

Chiton hanleyi Bean, 'Thorpe Brit. Mar. Conch., Suppl., 1844, p. 263, fig. 57. "Scarborough [, England]."

Mass.- Stellwagen Bank, Massachusetts Bay, 38 fathoms.

Hanleya mendicaria (Mighels and Adams).

Pilsbry, Manual Conch., 1892, ser. 1, vol. 14, p. 1S, pl.4, figs. S2-S5. 
Chiton mendicarius Mighels and Adams, Boston Journ. Nat. Hist., 1842, vol. 4, p. 42, pl. 4, fig. 8. "Casco BaY."

Me.-Casco Bay, 30 fathoms; Frenchman's Bay, 25 to 30 fathoms; Eastport; Georges Bank.

\section{ISCHNOCHITONIDAE.}

* Chaetopleura apiculata (Say).

Pilsbry, Manual Conch., 1892, ser. 1, vol. 14, p. 35, pl. 13, figs. 75-79.

Chiton apiculatus Say, Amer. Conch., 1830, app., pt. 7, p. - . "South Carolina * * Charleston."

Mass.-Vineyard Sound and Buzzards Bay, 3 to 12 fathoms; Nantucket; Eastham.

Conn.- Off New London.

\section{* Tonicella marmorea (Fabricius).}

Pilsbry, Manual Conch., 1892, ser. 1, vol. 14, p. 41, pl. 10, figs. 8-15.

Chiton marmoreus Fabricius, Fauna Groenlandica, 1780, p. 420. [GREENLAND.]

Chiton fulminatus Couthouy, Boston Journ. Nat. Hist., 1838, vol. 2, p. 80, pl. 3, fig. 19 .

Me.-Casco Bay; Frenchman's Bay, low water to 50 fathoms; Eastport.

Mass.-Revere; Massachusetts Bay; off Baker's Island.

Conn.- From codfish taken off Stonington (Linsley).

Tonicella marmorea coerulea (Winkley).

Chiton marmoreus var. coeruleus Winkley, Nautilus, 1894, vol. 8, p. 78. "Eastrort, [Maine]."

ME.-Eastport. 
Tonicella blaneyi Dall.

Dall, Proc. Biol. Soc. Wash., 1905, vol. 18, p. 203. "ofF Ironbound Island, Frenchians Bay, *** Maine." Dall, Nautilus, 1905, vol. 19, p. 88, pl. 4.

Me.-Frenchman's Bay, off Ironbound Island, in 20 fathoms.

* Trachydermon albus (Linné).

Pilsbry, Manual Conch., 1893, ser. 1, vol. 15, p. 64.

Chiton albus Linné, Syst. Nat., ed. 12, 1767, vol. 1, p. 1107. "O. ISLANdico."

Ischnochiton (Trachydermon) albus Pilsbry, Manual Conch., 1892, ser. 1 , vol. 14 , p. 70 , pl. 7, figs. $35-38$.

Me.- Casco Bay; Frenchman's Bay, 6 to 10 fathoms; Eastport.

N. H.- Isles of Shoals.

Mass.

Trachydermon exaratus (G. O. Sars).

Verrill, Trans. Conn. Acad., 1884, vol. 6, p. 208, pl. 30, figs. 2-2b.

Lophyrus exaratus G. O. Sars, Moll. Reg. Arct. Norv., 1878, p. 113, pl. 8, fig. 1. "BoD $\phi . "$

Mass.-Off Marthas Vineyard, 101 to 192 fathoms.

* Trachydermon ruber (Linné).

Chiton ruber Linné, Syst. Nat., ed. 12, 1767, vol. 1, p. 1107. "Oceano septentrionali."

Ischnochiton (Trachydermon) ruber Pilsbry, Manual Conch., 1892, ser. 1, vol. 14 , p. 80 , pl. 7, figs. 50-56.

Me.-Casco Bay; Frenchman's Bay; Eastport.

Mass.-Revere; Swampscott.

R. I.- Off Watch Hill, 5 fathoms.

Conn.-Off New London, 8 fathoms. 
Trachydermon ruber index (Balch).

Tonicella ruber var. index Balch, Nautilus, 1906, vol. 20, p. 66. "Blue Hill Bay, [Marne]."

Me.-Blue Hill Bay, 12 fathoms.

\section{MOPALIIDAE.}

Plaxiphora atlantica (Verrill and Smith).

Pilsbry, Manual Conch., 1892, ser. 1, vol. 14, p. 313, pl. 65, figs. 73-75; pl. 66, figs. 18-24.

Placophora (Euplacophora) atlantica Verrill and Smith in Verrill, Amer. Journ. Sci., 1882, ser. 3, vol. 24, p. 365. “off Nantucket, 640 fathons." Verrill, Trans. Conn. Acad., 1884, vol. 6, p. 206, pl. 30, figs. 1-1b.

Mass.-Off Nantucket Island, 122 fathoms.

\section{ACANTHOCHITIDAE.}

*Amicula vestita (Broderip and Sowerby).

Pilsbry, Manual Conch., 1893, ser. 1, vol. 15, p. 43, pl. 8, figs. 23-26.

Chiton vestitus Broderip and Sowerby, Zool. Journ., 1829, vol. 4, p. 368. "Oceano Arctico."

Amicula emersonii Gould, Inv. Mass., 1870, p. 264, fig. 527.

Me.-Casco Bay; Frenchman's Bay.

Mass.- Massachusetts Bay, from fish.

APLACOPHORA.

\section{CHAETODERMATIDAE.}

\section{Chaetoderma nitidulum Lovén.}

Lovén, Öfvers. Kongl. Vet.-Akad. Förh., 1844, vol. 1, p. 116, pl. 2, "IN ARgilla FUndi 15-40 ORg. AD ORAS SUECIAE OCCIDENTalis." Verrill, Proc. Amer. Assoc. Adv. Sci., 1874, vol. 22, p. 347 , pl. 6 , fig. 6 .

Me.-Casco Bay, 48 to 64 fathoms (Verrill). 


\section{PELECYPODA.}

PRIONODESACEA.

\section{SOLEMYIDAE.}

\section{*Solemya velum Say.}

Say, Journ. Acad. Nat. Sci. Phila., 1822, ser. 1, vol. 2, p. 317, " sovthern coast [of the United States]." Gould, Inv. Mass., 1870, p. 48, fig. 371.

Solenomya velum Morse, Biol. Bull., 1913, vol. 25, p. 261.

Me.- Casco Bay.

Mass.-Common in Buzzards Bay, Vineyard Sound, and Massachusetts Bay.

R. I.- Narragansett Bay.

Conn.- Near New Haven; Stratford and Long Beach.

*Solemya borealis Totten.

Totten, Amer. Journ. Sci., 1834, ser. 1, vol. 26, p. 366, pl., fig. 1. "Vicinity of Newport, [R. I.]." Gould, Inv. Mass., 1870, p. 50, fig. 372 .

Solenomya borealis Morse, Biol. Bull., 1913, vol. 25, p. 261.

Me.-Casco Bay; Old Orchard.

Mass.-Chelsea Beach; Nahant; Annisquam; Coffin's Beach; Cuttyhunk.

R. I. - Newport.

Conn.- Stonington.

\section{NUCULIDAE.}

\section{*Nucula proxima truncula Dall.}

Dall, Trans. Wagner Free Inst. Sci., 1898, vol. 3, pt. 4, p. 574.

"Long Island Sound Northiward to Nova Scotia."

Nucula proxima Gould, Inv. Mass., 1870, p. 150, fig. 458.

ME. to Conn.- Common, in 2 to 30 fathoms. 
Nucula proxima ovata (Verrill and Bush).

Nucula proxima var. ovata Verrill and Bush, Proc. U. S. Nat. Mus., 1898, vol. 20, p. 852, pl. 81, fig. 6; pl. 88, fig. 5. “Vineyard. Sound, off Cuttyhunk."

Mass.- Vineyard Sound, off Cuttyhunk, in 18 fathoms.

*Nucula delphinodonta Mighels and Adams.

Mighels and Adams, Boston Journ. Nat. Hist., 1842, vol. 4, p. 40, pl. 4, fig. 5. "Casco Bay." Gould, Inv. Mass., 1870, p. 153, fig. 461 .

Me.-Casco Bay, 5 to 95 fathoms; Frenchman's Bay, 4 to 50 fathoms; Eastport, 10 to 100 fathoms.

Mass.- Off Cape Ann, 30 fathoms; off Gay Head, 19 fathoms; Revere; Duxbury.

R. I.- East of Block Island, 29 fathoms.

*Nucula tenuis (Montagu).

Gould, Inv. Mass., 1870, p. 149, fig. 457.

Arca tenuis Montagu, Test. Brit., Suppl., 1808, p. 56, pl. 29, fig. 1. "Near Dunbar, [Scotland]."

Me.- Casco Bay; Frenchman's Bay, 10 to 20 fathoms; Eastport, 15 to 20 fathoms.

Mass.- "Georges Banks."

Conn. - ?Stonington (Linsley).

Nucula subovata Verrill and Bush.

Verrill and Bush, Proc. U. S. Nat. Mus., 189S, vol. 20, p. 852, pl. 81, fig. 8; pl. S3, fig. 5. “BETWEen N. LAT. $40^{\circ}$, W. LONG. $71^{\circ}$ $14^{\prime} 30^{\prime \prime}$, AND N. LAT. $37^{\circ} 8^{\prime}$, W. LONG. $74^{\circ} 33^{\prime}$, IN 157 To 444 FATHoMs."

South of Marthas Vineyard, 157 fathoms. 


\section{LEDIDAE.}

*Leda tenuisulcata (Couthouy).

Gould, Inv. Mass., 1870, p. 161, fig. 468.

Nucula tenuisulcata Couthouy, Boston Journ. Nat. Hist., 1838, vol. 2, p. 64, pl. 3, fig. 8. "MAss. BAY."

Me.-Casco Bay; Frenchman's Bay, 10 to 35 fathoms; Eastport, 10 to 35 fathoms.

N. H.- Off Isles of Shoals, 20 fathoms.

Mass.-From fish eaught off Nahant; Provincetown; Duxbury; Georges Bank, 40 to 150 fathoms.

R. I.- Newport.

\section{Leda acuta (Conrad).}

Nucula acuta Conrad, Amer. Marine Conch., 1832, p. 32, pl. 6, fig. 3. "Virginia" (fossil).

Leda unca Verrill, Proc. U. S. Nat. Mus., 1881, vol. 3, p. 401.

Seventy-three miles south of Marthas Vineyard, 65 to 219 fathoms.

R. I.- South of Newport, 85 to 155 fathoms.

Leda caudata (Donovan).

Gould, Inv. Mass., 1870, p. 165, fig. 471. Verrill and Bush, Proc. U. S. Nat. Mus., 1898, vol. 20, p. 855, pl. \$2, fig. 1.

Arca caudata Donovan, Brit. Shells, 1801, vol. 3, pl. 78. "KentISH COAST, [ENGLAND]."

Gulf of Maine, N. lat. $42^{\circ} 57^{\prime}$, W. long. $69^{\circ} 50^{\prime}$, 102 fathoms.

\section{Leda pernula (Müller).}

Verrill and Bush, Proc. U. S. Nat. Mus., 1898, vol. 20, p. 855, pl. 82, fig. 2.

Arca pernula Müller, Beschäft. Berlin. Naturf. Ges., 1779, vol. 4, p. 57. [Locality?]

Leda jacksonii Gould, Inv. Mass., 1870, p. 163, fig. 469 (in part). 
In 216 fathoms, 95 miles SSW. of Marthas Vineyard, also in 95 fathoms off Halifax, N. S.

\section{*Yoldia limatula (Say).}

Gould, Inv. Mass., 1870, p. 154, fig. 462.

N'ucula limatula Say, Amer. Conch., 1831, pt. 2, pl. 12 and explanatory pages. "Nahant, Massachusetts."

Me.-Casco Bay, 2 to 12 fathoms; Somes Sound, Mt. Desert; Eastport, 6 fathoms.

MAss.-Salem and Boston Harbor, 1 to 10 fathoms; Vineyard Sound and Buzzards Bay.

Conv.- Stonington from codfish (Linsley); off New Haven, 4 to 6 fathoms.

*Yoldia sapotilla (Gould).

Gould, Inv. Mass., 1870, p. 159, fig. 466.

Nucula sapotilla Gould, Inv. Mass., 1841, p. 100, fig. 61. "VICinity of CaPf Cod *** Provincetown harbour."

Me.-- Casco Bay; Frenchman's Bay, 15 to 35 fathoms; Eastport, 10 fathoms.

MAss.-Massachusetts Bay; Provincetown; off Gay Head, 19 to 25 fathoms; Duxbury.

R. I.- East of Block Island, 19 to 25 fathoms.

*Yoldia myalis (Couthouy).

Gould, Inv. Mass., 1870, p. 160, fig. 467.

Nucula myalis Couthouy, Boston Journ. Nat. Hist., 1838, vol. 2, p. 62, pl. 3, fig. 7. " COASt Of New England."

Me.- Casco Bay; Frenchman's Bay, 10 to 15 fathoms; Eastport, 10 to 20 fathoms.

Mass. - Massachusetts Bay, from fish. 
Yoldia cascöensis (Mighels and Adams).

Nucula cascöensis Mighels and Adams, Boston .Journ. Nat. Hist., 1842, vol. 4, p. 40, pl. 4, fig. 6. "CAsco BAY."

ME.- Casco Bay, from stomach of haddock.

*Yoldia (Portlandia) thraciaeformis (Storer).

Nucula thraciaeformis Storer, Boston Journ. Nat. Hist., 1838, vol. 2, p. 122, fig. "From the stomach of a Platessa *** Caught off Provincetown, Cape Cod."

Yoldia thraciaeformis Gould, Inv. Mass., 1870, p. 157, fig. 465.

Megayoldia thraciaeformis Verrill and Bush, Amer. Journ. Sci., 1897, ser. 4, vol. 3, p. 55, fig. 17.

ME-Casco Bay, 15 fathoms; Frenchman's Bay, 10 to 28 fathoms; Eastport, 10 fathoms.

Mass. - Off Cape Cod, in about 30 fathoms.

Yoldia (Microyoldia) regularis (Verrill).

Yoldia regularis Verrill, Trans. Conn. Acad., 1884, vol. 6, p. 228. " off Martha's Vineyard, in 349 fathoms."

Microyoldia regularis Verrill and Bush, Amer. Journ. Sci., 1897, ser. 4, vol. 3, p. 56, figs. 5, 6 .

MAss. - Oft' Thatcher's Island, 98 fathoms.

*Yoldia (Yoldiella) lucida (Lovén).

Yoldia lucida Lovén, Öfvers. Kongl. Vet.-Akad. Förh., 1846, vol. 3, p. 188. "BoH[ts]-FinM[ARK]."

Yoldia obesa Gould, Inv. Mass., 1870, p. 155, fig. 463.

Yoldiella lucida Verrill and Bush, Proc. U. S. Nat. Mus., 1898, vol. 20, p. 861, pl. 77, fig. 2; pl. 80 , fig. 3 .

Me.- Casco Bay and off Cape Elizabeth, 30 to 95 fathoms.

Mass.- Massachusetts Bay; near the Georges Bank, 110 to 150 fathoms.

R. I.- East of Block Island, 29 fathoms. 
Yoldia (Yoldiella) iris stricta (Verrill and Bush).

Yoldiella iris var. stricta Verrill and Bush, Proc. U. S. Nat. Mus., 1898, vol. 20, p. \$64, pl. 80, fig. 1. " off Cape Sable, 90 fathons."

Gulf of Maine, off Cape Sable, 90 fathoms.

Yoldia (Yoldiella) inflata (Verrill and Bush).

Yoldiella inflata Verrill and Bush, Amer. Journ. Sci., 1897, ser. 4, vol. 3, p. 56, figs. 3, 4, 11. "south of Georges' Bank to CAPE HatTERAS."

Georges Bank, N. lat. $41^{\circ} 53^{\prime}$, W. long. $66^{\circ} 35^{\prime}, 75$ fathoms.

Yoldia (Yoldiella) subangulata (Verrill and Bush).

Yoldiella subangulata Verrill and Bush, Proc. U. S. Nat. Mus., 1898, vol. 20 , p. 865 , pl. 77 , fig. 3 ; pl. 79 , fig. 6 . [OFF IsLeS of ShoAls.]

N. H.- Off Isles of Shoals, 51 fathoms.

Yoldia (Yoldiella) lenticula amblia (Verrill and Bush).

Yoldiella lenticula (Möller) var. amblia Verrill and Bush, Proc. U. S. Nat. Mus., 1898, vol. 20, p. 866, pl. 80, fig. 9; pl. 81, fig. 4 . "NORTh OF CAPE CoD."

Mass.- North of Cape Cod, 110 to 122 fathoms.

Yoldia (Yoldiella) fraterna (Verrill and Bush).

Yoldiella fraterna Verrill and Bush, Proc. U. S. Nat. Mus., 1898, vol. 20 , p. 867 , pl. 80 , fig. 5 ; pl. 82, fig. 8. " веTweEN N. LAT. $47^{\circ} 40^{\prime}$, W. LONG. $47^{\circ} 35^{\prime} 30^{\prime \prime}$, AND N. LAT. $37^{\circ} 8^{\prime}$, W. LONG. $74^{\circ} 33^{\prime}$."

Yoldia frigida Verrill, Trans. Conn. Acad., 1884, vol. 6, p. 279 (in part; non Torell).

Off the New England coast in 90 to 1608 fathoms.

Yoldia (Yoldiella) frigida Torell.

Yoldia frigida Torell, Bidrag Spitz. MIoll., 1859, p. 148, pl. 1, fig. 3. 
[Spitzbergen.] Verrill, Trans. Conn. Acad., 1882, vol. 5, p. 573, pl. 44, fig. 2.

Yoldiella frigida Verrill and Bush, Proc. U. S. Nat. Mus., 1898, vol. 20 , p. 872 , pl. 79 , fig. 4 .

Gulf of Maine and Jeffrey's Ledge, 88 to 92 fathoms.

Mass.- Off Cape Cod, 106 fathoms; off Marthas Vineyard, 153 to 312 fathoms.

Yoldia (Yoldiella) inconspicua (Verrill and Bush).

Yoldiella inconspicua Verrill and Bush, Proc. U. S. Nat. Mus., 1898 , vol. 20 , p. 869 , pl. 79 , figs. 3 , 5 . “N. LAT. $42^{\circ} 33^{\prime}$, W. LONG. $69^{\circ} 58.5^{\prime}$, AND N. LAT. $35^{\circ} 12^{\prime} 10^{\prime \prime}$, W. LONG. $74^{\circ} 57^{\prime} 15^{\prime \prime}$, IN 100 TO 705 FATHOMS."

Mass.- Off Marthas Vineyard, 100 to 705 fathoms.

\section{LIMOPSIDAE.}

Limopsis minuta (Philippi).

Verrill, Trans. Conn. Acad., 1882, vol. 5, p. 576. Verrill and B ush, Proc. U. S. Nat. Mus., 1898, vol. 20, p. 846, pl. 75, fig. 1; pl. 78 , fig. 7 .

Pectunculus minutus Philippi, Enum. Moll. Siciliae, 1836, vol. 1, p. 63 , pl. 5, fig. 3. [SiciLy.]

MAss.- South of Marthas Vineyard, 93 to 715 fathoms.

\section{Limopsis affinis Verrill.}

Verrill, Trans. Conn. Acad., 1885, vol. 6, p. 442. [off Marthas Vineyard.] Verrill and Bush, Proc. U.S. Nat. Mus., 1S98, vol. 20, p. 846 , pl. 75 , fig. 2.

Mass.- South of Marthas Vineyard, 197 fathoms. 
Limopsis sulcata Verrill and Bush.

Verrill and Bush, Proc. U. S. Nat. Mus., 1898, vol. 20, p. 845, pl. 92, fig. 2; pl. 95, fig. 9; pl. 96, fig. 1. [off Nantucket Shoals.]

Mass.- South of Nantucket Shoals, 64 to 349 fathoms.

Limopsis cristata Jeffreys.

Jeffreys, Ann. Mag. Nat. Hist, 1876, ser. 4, vol. 18, p. 434 . “ofF ** Ireland ***Bay of Biscay *** North Sea." Verrill, Proc. U. S. Nat. Mus., 1881, vol. 3, p. 402.

Mass.-South of Marthas Vineyard, 65 to 155 fathoms (Verrill).

\section{ARCIDAE.}

*Arca (Noëtia) ponderosa Say.

Tryon, Struct. Syst. Conch., 1884, vol. 3, p. 255, pl. 128, figs. 84,85 .

Arca ponderosa Say, Journ. Acad. Nat. Sci. Phila., 1822, ser. 1, vol. 2, p. 267. " southern coast [of the United States]."

Mass.-Chatham; Monomoy Point; Edgartown, Marthas Vineyard.

\section{* Arca (Scapharca) campechiensis pexata Say.}

Arca pexata Say, Journ. Acad. Nat. Sci. Phila., 1822, ser. 1, vol. 2, p. 268. "COAst of the United States." Gould, Inv. Mass., 1870 , p. 147, fig. 456.

Arca campechiensis Gmelin, Syst. Nat., 1790, vol. 1, pt. 6, p. 3312. Scapharca (Argina) campechensis Dall, Trans. Wagner Free Inst. Sci., 1898, vol. 3, pt. 4, p. 650.

Mass.-Provincetown; Chatham; Vineyard Sound; Buzzards Bay; Nantucket.

R. I.- Narragansett Bay; near Watch Hill.

Conn.- New Haven; Stratford; Branford. 
*Arca (Scapharca) transversa' Say.

Arca transversa Say, Journ. Acad. Nat. Sci. Phila., 1822, ser. 1, vol. 2, p. 269. "COAst of the United States." Gould, Inv. Mass., 1870, p. 148, fig. 456a.

Mass.-Chatham; Buzzards Bay; Marthas Vineyard; Nantucket; Wellfleet.

R. I.- Narragansett Bay; Greenwich Bay; near Watch Hill.

Conn.-Off New London; New Haven; Stratford.

\section{Arca (Bathyarca) pectunculoides Scacchi.}

Arca pectunculoides Scacchi, Test. Napolitani, 1832; Ann. Civ. d. Sicil., 1836, vol. 6, p. 82 . Verrill, Trans. Conn. Acad., 1882, vol. 5, p. 573, pl. 44, figs. 5,6 .

MIE.-Off Casco Bay, 94 fathoms; Cashe's Ledge, 27 to 90 fathoms; Gulf of Maine, 110 to 150 fathoms.

Mass.- Off Cape Cod, 92 to 122 fathoms; Georges Bank; south of Marthas Vineyard, 76 to 506 fathoms.

Arca (Bathyarca) pectunculoides septentrionalis (i. O. Wars.

Arca pectunculoides var. septentrionalis G. O. Sars, Moll. Reg. Arct. Norv., 1878, p. 43, pl. 4, fig. 2. "Hasvig [Norway.]"

Mass.- South of Marthas Vineyard, 79 to 500 fathoms (Verrill).

\section{Arca (Bathyarca) pectunculoides frielei Friele.}

Arca frielei Friele (ex Jeffreys Ms.), Nyt Mag. f. Naturv., 1877, vol. 23, pt. 3, p. 2. [North Atlantic.] Jeffreys, Proc. Zool. Soc. London, 1879, p. 573, pl. 45, figs. 4, $4 a$.

Arca pectunculoides var. frielei Verrill, Trans. Conn. Acad., 1882, vol. 5, p. 574.

Mass.- South of Marthas Vineyard, 100 to 487 fathoms. 
Arca (Bathyarca) pectunculoides crenulata Verrill.

Verrill, Trans. Conn. Acad., 1882, vol. 5, p.575. "off Martha's VINEYARD."

MAss.- Off Marthas Vineyard, 85 to 120 fathoms.

Arca (Bathyarca) anomala (Verrill and Bush).

Bathyarca anomala Verrill and Bush, Proc. U. S. Nat. Mus., 1898, vol. 20, p. 844, pl. 77, fig. 8. "off Cashes Ledge, [Gulf of Maine]."

Me.-Off Cashes Ledge, Gulf of Maine, 27 fathoms.

\section{PTERIIDAE.}

Pteria hirundo vitrea (Reeve).

Dall, Trans. Wagner Free Inst. Sci., 1898, vol. 3, pt. 4, p. 670.

Avicula vitrea Reeve, Conch. Icon., Avicula, 1857, vol. 10, pl. 18, fig. 68. "WEst Indies."

Avicula hirundo var. nitida Verrill, Proc. U. S. Nat. Mus., 1881, vol. 3, p. 402; Trans. Conn. Acad., 1882, vol. 5, p. 582, pl. 58, fig. 43.

MAss. - South of Marthas Vineyard; found in considerable numbers adhering to hydroids, in 65 to 192 fathoms (Verrill).

\section{OSTREIDAE.}

*Ostrea virginica Gmelin.

The Oyster.

Gmelin, Syst. Nat., 1790, vol. 1, pt. 6, p. 3336. "Oceano AMERICANO ET INDICO."

Ostrea virginiana Gould, Inv. Mass., 1870, p. 202. Brooks, The Oyster, Baltimore, ed. 2, 1905.

Ostrea borealis Lamarck, Hist. Nat. Anim. sans Vert., 1819, vol. 6, pt. 1, p. 204. 
O. virginica var. meadii Davis, Apteryx, 1905, vol. 1, p. 117, pl. 9, fig. E (young).

Formerly living in Casco Bay and Damariscotta, Me.; once abundant in various parts of Massachusetts and Cape Cod Bays. Still living in Oyster River, Great Bay, N. H. Common in Vinerard Sound, Buzzards Bay, Narragansett Bay, and Long Island Sound. Southern oysters have been planted for a number of years in the waters of R. I. and Conn.

\section{UNIONIDAE:}

*Lampsilis ventricosa (Barnes).

Simpson, Descriptive Cat. Naiades, 1914, p. 38.

Unio ventricosus Barnes, Amer. Journ. Sci., 1823, ser. 1, vol. 6, p. 267, pl. 13, fig. 14. “ THE Wisconsan *** Mississippi, NEAR Prairie du Chien."

Unio occidens Lea, Trans. Amer. Phil. Soc., 1830, new ser., vol. 3, p. 435, pl. 10, fig. 16.

VT.-Larrabee's Point; Shoreham; Chimney Point, Lake Champlain.

\section{*Lampsilis cariosa (Say).}

Simpson, Descriptive Cat. Naiades, 1914, p. 43.

Unio cariosus Say, Nich. Encycl., 1816, vol. 2, pl. 3, fig. 2. “Delaware and Schuylkill Rivers." Gould, Inv. Mass., 1870, p. 172, fig. 475 .

Me.-Warren; Chickawaukee Pond; Perham Lake.

Mass.-Connecticut River; Haverhill; ponds in Plymouth Co.

Conn.- Connecticut and Housatonic Rivers.

\section{${ }^{*}$ Lampsilis ochracea (Say).}

Simpson, Descriptive Cat. Naiades, 1914, p. 49.

Unio ochraceus Say, Nich. Encycl., 1816, vol. 2, pl. 3, fig. 8. "Delaware and Schuylkill Rivers." 
ME.-Orono; South Pond, Warren; Waldoboro; Chickawaukee Pond, Knox Co.

Mass.- Ponds of Plymouth Co.; Hatchville.

Conn.- Housatonic River; Connecticut River.

${ }^{*}$ Lampsilis luteola rosacea (DeKay).

Simpson, Descriptive Cat. Naiades, 1914, p. 62.

Unio rosaceus DeKay, Nat. Hist. N. Y., Moll., 1843, p. 192, pl. 39, figs. 355, 356. "SEneca Lake, [N. Y.]"

VT.-Creek near Mallett's Bay, Lake Champlain (Perkins).

*Lampsilis radiata (Gmelin).

Simpson, Descriptive.Cat. Naiades, 1914, p. 64.

Mya radiata Gmelin, Syst. Nat., 1790, vol. 1, pt. 6, p. 3220 . “ IN Malabariae fluvits."

Unio radiatus Gould, Inv. Mass., 1870, p. 170, fig. 474.

ME. to Conn. - Common in most of the larger streams and lakes.

${ }^{*}$ Lampsilis recta (Lamarck).

Simpson, Descriptive Cat. Naiades, 1914, p. 95.

Unio recta Lamarck, Hist. Nat. Anim. sans Vert., 1819, vol. 6, pt. 1, p. 74. "Lac Erie."

Eurynea praelonga Stimpson, Shells of New England, 1851, p. 13.

VT.-Shoreham; Chimney Point, Lake Champlain.

\section{*Lampsilis nasuta (Say).}

Simpson, Descriptive Cat. Naiades, 1914, p. 97.

Unio nasutus Say, Nich. Encycl., 1816, vol. 2, pl. 4, fig. 1. “Delaware and Schuylkill Rivers."

Eurynea nasuta Stimpson, Shells of New England, 1851, p. 13.

ME.

N. H.- Hampstead; Kingston. 
Mass.- Wayland; Woburn; Newton; Fresh Pond, Cambridge; Plymouth; Concord.

R. I.- Providence; Rhodes; Warwick.

ConN.- - Stonington; Hartford.

* Lampsilis (Proptera) alata (Say).

Simpson, Descriptive Cat. Naiades, 1914, p. 162.

Unio alatus Say, Nich. Encycl., 1816, vol. 2, pl. 4, fig. 2. "LAkE ERIE."

Metaptera alata Stimpson, Shells of New England, 1851, p. 14.

VT.-Larrabee's Point; Chimney Point, Lake Champlain.

* Lampsilis (Proptera) gracilis (Barnes).

Simpson, Descriptive Cat. Naiades, 1914, p. 181.

Unio gracilis Barnes, Amer. Journ. Sci., 1823, ser. 1, vol. 6, p. 274. "The Wisconsan *** AND ThE LAKES".

Hetaptera gracilis Stimpson, Shells of New England, 1S5̃1, p. 14.

VT.-Larrabee's Point; Shoreham; Chimney Point, Lake Champlain.

\section{Strophitus edentulus (Say).}

Simpson, Descriptive Cat. Naiades, 1914, p. 345.

Alasmodonta edentula Say, New Harmony (Ind.) Disseminator, 1829, vol. 2, no. 22, p. 340. "WABAsh River."

Anodon undulata Gould, Inv. Mass., 1870, p. 182, fig. 482 (non Say).

VT. - Lake Champlain and its tributary streams.

MAss. - Blackstone River and its tributaries; Westfield.

Conn.- Connecticut River at Hartford.

Strophitus undulatus (Say).

Simpson, Descriptive Cat. Naiades, 1914, p. 349. 
Anodonta undulata Say, Nich. Encycl., 1816, vol. 2, pl. 3, fig. 5 . [United States.]

Me.-Androscoggin River at Bethel; Warren; Aroostook Co. N. H.- Connecticut River at Hanover.

VT.-Connecticut River at Hartland.

Mass.-Ware; Uxbridge; Bullow's Pond, Newton.

R. I.-Warwick; Providence; North Smithfield.

Conn.- Housatonic River.

\section{*Anodonta cataracta Say.}

Say, Nich. Encycl., 1816, vol. 2, pl. 3, fig. 4. [United States.] Simpson, Descriptive Cat. Naiades, 1914, p. 386.

Anodon fluviatilis Gould, Inv. Mass., 1870, p. 178, fig. 480.

ME. to Conn.-Common in most of the lakes and ponds.

*Anodonta marginata Say.

Say, Nich. Encycl., 1816, vol. 2, pl. 3, fig. 5. [United States.] Simpson, Descriptive Cat. Naiades, 1914, p. 388.

Anodonta fragilis Lamarck, Hist. Nat. Anim. sans Vert., 1819, vol. 6 , pt. 1, p. 85 .

Anodonta lacustris Lea, Journ. Acad. Nat. Sci. Phila., 1860, ser. 2 , vol. 4, p. 363, pl. 62, fig. 188.

Me.-Salmon Brook Lake, Perham (Nylander); Warren (Lermond).

VT.-Wallingford; Otter Creek.

\section{*Anodonta implicata Say.}

Say, New Harmony (Ind.) Disseminator, 1929, vol. 2, no. 22, p. 310. "Danvers, Mass." Simpson, Descriptive Cat. Naiades, 1914 , p. 391.

Anodonta nowtoniensis Lea, Trans. Amer. Phil. Soc., 1839, new ser., vol. 6, p. 79, pl. 21, fig. 66 . 
Anodonta housatonica Linsley, Amer. Journ. Sci., 1845, ser. 1, vol. 48 , p. 277.

Anodon implicata Gould, Inv. Mass., 1870, p. 180, fig. 481.

ME. - Warren (Lermond); pond near Eastport (Mighels).

Mass.- Agawam River, East Wareham; Mystic Lake, Arlington.

R. I:- Cunliff Pond, Providence; Warwick; North Smithfield. Conn.- Hartford.

*Anodonta grandis benedictensis (Lea).

Simpson, Descriptive Cat. Naiades, 1914, p. 423.

Symphynota benedictensis Lea, Trans. Amer. Phil. Soc., 1837, vol. 5, p. 104, pl. 16, fig. 4S. "Lake Champlain."

VT.-Larrabee's Point; Chimney Point, Lake Champlain.

\section{Anodontoides ferussacianus (Lea).}

Simpson, Descriptive Cat. Naiades, 1914, p. 467.

Anodonta ferussaciana Lea, Trans. Amer. Phil. Soc., 1837, new ser., vol. 5, p. 45, pl. 6, fig. 15. "Ohio River, Near Cincinnatr."

Conn.- Whitneyville (Linsley).

\section{Symphynota compressa Lea.}

Lea, Trans. Amer. Phil. Soc., 1830, new ser., vol. 3, p. 450, pl. 12; fig. 22. "Ohio *** Norman's Kill, near Albany." Simpson, Descriptive Cat. Naiades, 1914, p. 481.

VT.-- Streams west of the Green Mountains (Adams).

\section{Symphynota compressa plebeia (C. B. Adams).}

Simpson, Descriptive Cat. Naiades, 1914, p. 483.

Unio compressus var. plebeius C. B. Adams, Thompson's Hist. Vt., 1842, pt. 1, p. 166. "Middlebury, [VT.]"

VT.-Middlebury (Adams); creek near Mallett's Bay (Perkins).

Conn.- New Haven, canal. 
*Symphynota costata (Rafinesque).

Simpson, Descriptive Cat. Naiades, 1914, p. 488.

Alasmidonta costata Rafinesque, Ann. Gén. Sci. Phys. Bruxelles, 1820, vol. 5, p. 318, pl. 82, figs. 15, 16. [Kentucky River.]

Alasmodonta rugosa Barnes, Amer. Journ. Sci., 1823, ser. 1, vol. 6, p. 278, pl. 13, fig. 21. C. B. Adams, Thompson's Hist. Vt., 1842, pt. 1 , p. 165 .

VT.-Chimney Point, Lake Champlain and streams west of the Green Mountains (Adams).

\section{*Alasmidonta undulata (Say).}

Simpson, Descriptive Cat. Naiades, 1914, p. 494.

Monodonta undulata Say, Nich. Encycl., 1816, vol. 2, pl. 3, fig. 3.

"Delaware and Schuylkill Rivers."

Margaritana undulata Gould, Inv. Mass., 1870, p. 176, fig. 478.

Me.-Eagle Lake; Fish River; Androscoggin River; Rangeley; Warren.

N. H.-Concord; Connecticut, Hanover.

VT.- Hartland.

Mass-Milton; Sudbury; Wellesley; Andover; Blackstone River, Uxbridge.

R. I.- Blackstone River, Pawtucket; Warwick.

Conn.-Connecticut and Housatonic River; Meriden.

\section{*Alasmidonta heterodon (Lea).}

Simpson, Descriptive Cat. Naiades, 1914, p. 499.

Unio heterodon Lea, Trans. Amer. Phil. Soc., 1830, new ser., vol. 3, p. 428, pl. 8, fig. 11. " Schuylkill and Derby Creek, PA."

N. H.-Connecticut River, at Hanover and Cornish.

VT.-Connecticut River, at Hartland.

Mass. - Westfield.

Conn.-Connecticut River; Little River; Housatonic River; Meriden. 
*Alasmidonta marginata (Say).

Simpson, Descriptive Cat. Naiades, 1914, p. 504.

Alasmodonta marginata Say, Nich. Encycl., 1816, vol. 2, pl. 3, fig. 5. [United States.]

Margaritana marginata Gould, Inv. Mass., 1870, p. 177, fig. 479.

ME.-Westbrook, Dennys River.

N. H.-Connecticut River, Hanover.

VT.-Otter Creek, Wallingford.

Mass.-Blackstone River, Andover; West Brookfield; Sherborn.

R. I.-Cunliff Pond, Providence.

Conn.- Housatonic River, Derby.

*Unio complanatus (Dillwyn).

Gould, Inv. Mass., 1870, p. 167, fig. 472. Simpson, Descriptive Cat. Naiades, 1914, p. 651.

Mya complanata Dillwyn, Catalogue, 1817, vol. 1, p. 51. "RIVers in Virginia. $* * *$ Maryland and New Jersey. $* * *$ MissisSIPPI."

ME. to Conn.- Common in almost every stream and pond.

Unio complanatus mainensis Rich.

Rich, Science, 1915, new ser., vol..42, p. 580. "Songo Pond, about three miles south of Bethel, Me."

ME.-Ponds and lakes of Oxford Co.

\section{*Unio roanokensis northamptonensis Lea.}

Simpson, Descriptive Cat. Naiades, 1914, p. 668.

Unio northamptonensis Lea, Proc. Acad. Nat. Sci. Phila., 1861, p. 392. "Connecticut River, at Northampton. At SpringField *** Below Hartford *** Neuse River, N. C." Lea, Journ. Acad. Nat. Sci. Phila., 1862, ser. 2, vol. 5, p. 190, pl. 25, fig. 260 .

Mass.- Northampton; Springfield.

Conn.- Connecticut River below Hartford. 


\section{MARGARITANIDAE.}

*IMargaritana margaritifera (Linné).

Simpson, Descriptive Cat. Naiades, 1914, p. 513.

Mya margaritifera Linné, Syst. Nat., ed. 10, 1758, vol. 1, p. 671. "IN TOTIUS ORBIS ARCTICI CATARACTIS."

Margaritana arcuata Gould, Inv. Mass., 1870, p. 174, fig. 477.

ME.-Aroostook Co.; Scarboro; Warren; Hebron.

VT. - Winooski River.

Mass.-Haydenville; Southampton; Lunenburg; Ware.

R. I. - Exeter.

Conn.-- Turnbull River (Linsley); Waterford; Bristol; Granby.

\section{PECTINIDAE.}

*Pecten (Chlamys) islandicus Müller.

Pecten islandicus Müller, Zool. Danicae Prodr., 1776, p. 248. [DENMARK.]

Chlamys islandica Verrill, Trans. Conn. Acad., 1897, vol. 10, p. 72, pl. 16, figs. 2-5b; pl. 20, fig. 9; pl. 21, fig. 2; var. insculpta Verrill, ibid., p. 73.

Me. I Casco Bay, 20 fathoms; Frenchman's Bay, 25 to 45 fathoms; Eastport.

MAss.- Massachusetts Bay; off Marthas Vineyard, 69 to 194 fathoms; Georges Bank, 40 to 65 fathoms.

Conn.- Stonington in an eel-pot (Linsley).

Pecten (Chlamys) costellatus (Verrill and Bush).

Chlamys costellata Verrill and Bush in Verrill, Trans. Conn. Acad., 1897, vol. 10, p. 75. “ off the COAST of Newfoundland IN 67 то 72 FATHOMs."

Mass.-Off Half-way Rock, Marblehead, in 30 fathoms (Balch).

Pecten (Aequipecten) glyptus Verrill.

Pecten glyptus Verrill, 'Trans. Conn. Acad., 1882, vol. 5, p. 580. " off Martia's Vineyard." 
Pecten tryoni Dall, Bull. Mus. Comp. Zoöl., 1SS9, vol. 1S, p. 4.3S,

Chlamys (Aequipecten) glypta Verrill, Trans. Conn. Acad., 1897. vol. 10 , p. 76 , pl. 16, figs. $7-11$.

Mass.- Off Marthas Vineyard, 85 to 120 fathoms.

*Pecten (Plagioctenium) gibbus borealis Say.

Ostrea gibba Linné, Syst. Nat., ed. 10, 1758, vol. 1, p. 698.

Pecten borealis Say, Journ. Acad. Nat. Sci. Phila., 1822, ser. 1, vol. 2, p. 260. " New England."

Pecten irradians Gould, Inv. Mass., 1870, p. 199, fig. 496 (non Lamarck).

Pecten gibbus var. borealis Dall, Trans. Wagner Free Inst. Sci., 1898, vol. 3, pt. 4, p. 747.

Mass. to Conn.- The common scallop of southern New England.

*Pecten (Placopecten) magellanicus (Gmelin).

Dall, Trans. Wagner Free Inst. Sci., 1898, vol. 3, pt. 4, p. 726.

Ostrea magellanica Gmelin, Syst. Nat., 1790, vol. 1, pt. 6, p. 3317.

"Freto Miagellanico."

Pecten tenuicostatus Mighels and Adams, Boston Journ. Nat. Hist., 1842, vol. 4, p. 41, pl. 4, fig. 7. Gould, Inv. Mass., 1870, p. 196, fig. 494.

Pecten fuscus Linsley, Amer. Journ. Sci., 1845, ser. 1, vol. 48, p. 278.

Pecten clintonius Verrill, Trans. Conn. Acad., 1884, vol. 6, p. 261 (non Say).

ME.-Casco Bay, 4 to 10 fathoms; Frenchman's Bay, 3 to 10 fathoms; Eastport.

Mass.-Massachusetts Bay; Duxbury; Vineyard Sound. Georges Bank, 45 fathoms.

R. I.-Off Watch Hill; off Block Island; Point Judith.

Conn.-Stonington from codfish. 
Pecten (Camptonectes) vitreus (Gmelin).

Ostrea vitrea Gmelin, Syst. Nat., 1790, vol. 1, pt. 6, p. 3328. "Oceano septentrionali."

Pecten vitreus Verrill, Trans. Conn. Acad., 1882, vol. 5, p. 580, pl. 42, fig. 21.

Camptonectes (Palliolum) vitrea Verrill, Trans. Conn. Acad., 1897, vol. 10 , p. 65 , pl. 18 , figs. $6-14$.

Mass.-Off Marthas Vineyard in from 100 to 506 fathoms; northward in 57 to 400 fathoms, attached to various objects, chiefly to the gorgonian corals (Verrill).

Pecten (Camptonectes) striatus Müller.

Pecten striatus Müller, Zool. Danicae Prodr., 1776, p. 248; Zool. Danica, 1788, vol. 2, p. 26, pl. 60, figs. 3-5. [Denninkк.] Verrill, Trans. Conn. Acad., 1884, vol. 6, p. 233.

Camptonectes (Palliolum) striata Verrill, Trans. Conn. Acad., 1897, vol. 10, pp. 62, 66, pl. 18, figs. 14, $14 a$.

Mass.-Off Marthas Vineyard, 100 fathoms.

\section{Pecten (Pseudamusium) pustulosus Verrill.}

Pecten pustulosus Verrill, Amer. Journ. Sci., 1873, ser. 3, vol. 5, p. 14. “Near St. George's Bank" [and Gulf of Maine.]

Pecten hoskynsi var. pustulosus Verrill, Trans. Conn. Acad., 1882, vol. 5, p. 581, pl. 42, figs. 22, $22 a$.

Pecten (Pseudamusium) imbrifer Dall, Bull. Mus. Comp. Zoöl., 1886, vol. 12, p. 220 (in part; non Lovén).

Cyclopecten pustulosus Verrill, Trans. Conn. Acad., 1897, vol. 10, p. 83 , pl. 19, figs. 3,4 .

Gulf of Maine, 150 fathoms.

Mass.- South of Marthas Vineyard, 120 to 365 fathoms.

Pecten (Pseudamusium) subimbrifer (Verrill and Bush).

Cyclopecten subimbrifer Verrill and Bush in Verrill, Trans. Conn. Acad., 1897, vol. 10, p. 84. “OFF the EASTERn COAST of the 
United States." Verrill and Bush, Proc. U. S. Nat. Mus., 1898, vol. 20 , p. 840 , pl. 85 , figs. 8,9 .

Pecten hoskynsi Verrill, Trans. Conn. Acad., 1882, vol. 5, p. 581, pl. 44, fig. 11 (non Forbes, 1844).

Gulf of Maine, 115 to 150 fathoms.

Mass. - South of Marthas Vineyard, 120 to 365 fathoms.

\section{Pecten (Propeamusium) thalassinus Dall.}

Pecten (Pseudamusium) thalassinus Dall, Bull. Mus. Comp. Zoöl., 1886, vol. 12, p. 221. “OFf Martha's Vineyard” and “ofF Havanna."

Pecten fenestratus Verrill, Proc. U. S. Nat. Mus., 1881, vol. 3, p. 403 (non Forbes, 1844).

Propeamusium thalassinum Verrill, Trans. Conn. Acad., 1897, vol. 10 , p. 87 , pl. 19, figs. 5-7.

Mass.- Off Marthas Vineyard, 86 to 310 fathoms; most numerous in 93 to 100 fathoms (Verrill).

\section{LIMIDAE.}

Limaea subovata (Jeffreys).

Lima subovata Jeffreys, Ann. Mag. Nat. Hist., 1876, ser. 4, vol. 18, p. 427 . "OFF THE NORTH-IVESTERN COAST OF IRELAND $* * *$ BETWEEN THE Hebrides AND FäRÖE IsLES *** AND BETIEEEN the Färöes and Shetland ** * off the Azores * * * Palermo." Jeftreys, Proc. Zool. Soc. London, 1879, p. 563, pl. 45, fig. 2.

Limaea subrota (sic) Verrill, Trans. Conn. Acad., 1882, vol. 5, p. 580 .

Mass.-Off Marthas Vineyard, 100 to 500 fathoms (Verrill).

\section{ANOMIIDAE.}

*Anomia aculeata Mïller.

Müller, Zool. Danicae Prodr., 1776, p. 249. [Denmark.] Gould, Inv. Mass., 1870, p. 204, fig. 498. 
Me.-Common in Casco Bay and northward, low water to 80 fathoms.

Mass.- Off Gay Head, 10 fathoms; Duxbury.

Conn.- Off Stonington, 4 to 5 fathoms.

*Anomia simplex d'Orbigny.

d'Orbigny, Moll. Cubana, 1845, (Spanish ed.), vol. 2, p. 367, pl. 38, figs. 31-35. [CUBA.]

Anomia ephippium Gould, Inv. Mass., 1870, p. 204, fig. 497 (non Linné).

Anomia electrica Gould, Inv. Mass., 1870, p. 205, fig. 499 (non Linné).

Anomia squamula Gould, Inv. Mass., 1870, p. 206 (non Linné). Anomia glabra Verrill, Amer. Journ. Sci., 1872, ser. 3, vol. 3, p. 213.

Me. to Conn.- Common south of Cape Cod, adhering to oysters and scallops.

MYTILIDAE.

*Mytilus edulis Linné.

Linné, Syst. Nat., ed. 10, 1758, vol. 1, p. 705. “O. Europaeo, Indico \& Balthico." Gould, Inv. Mass., 1870, p. 183, fig. 483.

Me. to Conn.- The common edible mussel.

*Mytilus edulis pellucidus Pennant.

Gould, Inv. Mass., 1870, p. 184, fig. 484.

Mytilus pellucidus Pennant, Brit. Zool., 1777, vol. 4, p. 95, pl. 63, fig. 75. "Anglesea, [England.]"

ME. to Conn.-Common, associated with the typical form.

*Mytilus (Hormomya) recurvus Rafinesque.

Mytilus recurvus Rafinesque, Ann. Gén. Sci. Phys. Bruxelles, 1820, vol. 5, p. 54. "LE Mississippi PRÈs DE LA NouvelleOrléans." 
Mytillus hamatus Say, Journ. Acad. Nat. Sci. Phila., 1822, ser. 1, vol. 2, p. 265.

R. I.- Narragansett Bay.

Conn.- New Haven Harbor (Perkins, 1870). Introduced in the planting of southern oysters in these waters.

*Modiolus modiolus (Linné).

Lamarck, Prod. Nouv. Class. Coq., 1799, p. 87.

Mytilus modiolus Linné, Syst. Nat., ed. 12, 1767, vol. 1, p. 1158. "M. Mediterraneo, Norvegico."

Modiola modiolus Gould, Inv. Mass., 1870, p. 186, fig. 485.

ME. to Conn.- Common, from low water to 80 fathoms.

*Modiolus (Brachydontes) demissus plicatulus (Lamarck).

Dall, Trans. Wagner Free Inst. Sci., 1898, vol. 3, pt. 4, p. 795. Mytilus demissus Dillwyn, Catalogue, 1817, vol. 1, p. 314. "Virginia."

Modiola plicatula Lamarck, Hist. Nat. Anim. sans Vert., 1819, vol. 6, pt. 1, p. 113. No locality. Gould, Inv. Mass., 1870, p. 188, fig. 486.

Modiola plicatula truncata Davis, Apteryx, 1905, vol. 1, p. 117, pl. 9, fig. D.

Me.- Casco Bay; mouth of the Kennebec River.

Mass.-Salem; Revere; Chatham; Woods Hole; Edgartown.

R. I.-Oakland Beach; Buttonwoods; Wickford.

Conn.

\section{*Musculus substriatus (Gray).}

Modiola laevigata var. $\beta$. substriata Gray, Suppl. to Appendix to Parry's Voyage 1819-20, 1824, p. ccxlv. [Northwest PAssage.] 
Modiolaria discors Gould, Inv. Mass., 1870, p. 192, fig. 489 (non Linné).

Me.-Casco Bay; Frenchman's Bay, 15 to 20 fathoms; Eastport.

MAss.- Vineyard Sound, 14 to 25 fathoms.

R. I.

Conn.- Oyster River, near New Haven (Linsley).

*Musculus niger (Gray).

Modiola nigra Gray, Suppl. to Appendix to Parry's Voyage 1819-20, 1824, p. ecxliv. [Northwest Passage.]

Modiola nexa Gould, Inv. Mass., 1841, p. 128, fig. 86.

Modiolaria nigra Gould, Inv, Mass., 1870, p. 190, figs. 487, 488.

Mass.-Provincetown; Vineyard Sound, 10 to 15 fathoms.

Conn.- Stonington, in stomach of cod (Linsley).

*Musculus corrugatus (Stimpson).

Mytilus corrugatus Stimpson, Shells of New England, 1851, p. 12.

"Eastport to Cape Cod."

Modiolaria corrugata Gould, Inv. Mass., 1870, p. 193, fig. 491.

Me.-Casco Bay, 15 fathoms; Frenchman's Bay, 6 to 10 fathoms.

Mass.-Off Marthas Vineyard, 20 to 25 fathoms; off Nauset Light; Georges Bank.

Conn.--Off New London.

${ }^{*}$ Crenella glandula (Totten).

Gould, Inv, Mass., 1870, p. 194, fig. 492.

IIodiola glandula Totten, Amer. Journ. Sci., 1834, ser. 1, vol. 26, p. 367, fig. 3. "Provincetown Harbor, (Mass.)." 
Me.- Common from Casco Bay to Eastport in 3 to 60 fathoms. Mass. - Marblehead, 7 fathoms; Buzzards Bay and Vineyard Sound, 5 to 15 fathoms.

R. I.- Off Block Island, 29 fathoms.

Conv.- Stonington (Linsley); off New London.

Crenella pectinula (Gould).

Gould, Inv. Mass., 1870, p. 195, fig. 493.

Modiola pectinula Gould, Inv. Mass., 1841, p. 127, fig. 85. "ST. George's Bank."

Georges Bank.

*Crenella decussata (Montagu).

Verrill, Trans. Conn. Acad., 1882, vol. 5, p. 578, pl. 44, fig. 7.

Mytilus decussatus Montagu, Test. Brit., Suppl., 1803, p. 69. "SCOTISH COAST."

Me.-Casco Bay, 10 to 20 fathoms; Frenchman's Bay, 10 to 28 fathoms; Eastport, 4 to 40 fathoms.

N. H.- Off Isles of Shoals, 20 fathoms.

Mass.-Provincetown; off Marthas Vineyard, 64 to 115 fathoms; Duxbury.

ConN. - Stonington (Linsley).

\section{ANOMALODESMACEA.}

\section{PHOLADOMYACIDAE.}

Aporema arata (Verrill and Smith).

Dall, Trans. Wagner Free Inst. Sci., 1903, vol. 3, pt. 6, p. 1532. Pholadomya arata Verrill and Smith in Verrill, Amer. Journ. Sci., 1881, ser. 3, vol. 22, p. 301. [off Marthas Vineyard.]. Verrill, Trans. Conn. Acad., 1882, vol. 5, p. 567, pl. 58, fig. 37.

Mass.-South of Marthas Vineyard, 69 to 130 fathoms. 


\section{PERIPLOMIDAE.}

*Periploma fragilis (Totten).

Dall, Bull. U. S. Nat. Mus., 1889, no. 37, p. 64, pl. 59, fig. 7.

Anatina fragilis Totten, Amer. Journ. Sci., 1835, ser. 1, vol. 28, p. 348, fig. 1. "Newport Harbor, R. I."

Inatina papyracea Gould, Inv. Mass., 1870, p. 66, fig. 382 (non Say, 1822).

Me. - Casco Bay, 10 fathoms; Frenchman's Bay, 4 to 10 fathoms; Old Orchard.

Mass.- Massachusetts Bay.

R. I.- Off Block Island, 29 fathoms.

\section{*Periploma (Cochlodesma) leanum (Conrad).}

Dall, Bull. U. S. Nat. Mus., 1889, no. 37, p. 64, pl. 59, fig. 6.

Anatina leana Conrad, Journ. Acad. Nat. Sci. Phila., 1831, ser. 1, vol. 6, p. 263, pl. 11, fig. 11. " NORTHERn COAst of THE U. S."

Cochlodesma leanum Gould, Inv. Mass., 1870, p. 68, fig. 383.

ME.-Casco Bay; Frenchman's Bay; Eastport.

Mass.-Nahant; Cape Cod; Vineyard Sound; Nantucket; Georges Bank.

Conn.- Long Island Sound, 3 to 10 fathoms.

THRACIIDAE.

\section{*Thracia conradi Couthouy.}

Couthouy, Boston Journ. Nat. Hist., 1839, vol. 2, p. 153, pl. 4, fig. 2. "COASt of New England." Gould, Inv. Mass., 1870, p. 69, fig. 384. Morse, Nautilus, 1913, vol. 27, p. 73.

Thracia dectivis Conrad, Amer. Marine Conch., 1832(?), p.44, pl. 9, fig. 2 (non Pennant, 1778).

ME.-Casco Bay, 6 to 15 fathoms; Frenchman's Bay, 3 to 16 fathoms; Eastport, 6 fathoms. 
MAss.-Nahant; Revere; Vineyard Sound, 6 to 8 fathoms; Duxbury.

R. I.

Thracia truncata Mighels and Adams.

Mighels and Adams, Boston Journ. Nat. Hist., 1842, vol. 4, p. 38, pl. 4, fig. 1. "CAsco BAY, Me." Gould, Inv. Mass., 1870, p. 72 , fig. 386 .

Me.-Casco Bay, 10 to 20 fathoms; Frenchman's Bay, 20 to 25 fathoms; Eastport.

Mass.-Off Lynn; Massachusetts Bay; off Marthas Vineyard; Georges Bank.

R. I.- Off Block Island, 29 fathoms.

\section{*Thracia myopsis Möller.}

Möller, Krøyer's Naturh. Tidskr., 1842, vol. 4, p. 94. [GreENLAND.] Gould, Inv. Mass., 1870, p. 71, fig. 385.

Thracia couthouyi Stimpson, Shells of New England, 1S51, p, 23.

Me.-Casco Bay; Frenchman's Bay, 10 to 12 fathoms: Eastport.

Mass-Massachusetts Bay; Georges Bank.

\section{PANDORIDAE.}

Pandora (Kennerleyia) brevis (Verrill and Bush).

Kennerlia brevis Verrill and Bush, Proc. U. S. Nat: Mus., 1898, vol. 20 , p. 821 , pl. 88 , figs. $7 a, 7 b$.

Kennerlia glacialis Verrill, Proc. U. S. Nat. Mus., 1880, vol. 3, p. 397 (non Leach, 1819). [off Marthas Vineyard.]

Mass.-Off Marthas Vineyard, 45 to 100 fathoms.

*Pandora (Clidiophora) gouldiana Dall.

Dall, Bull. Mus. Comp. Zoöl., 1886, vol. 12, p. 312. "New England." 
Pandora tritincata Conrad, Amer. Marine Conch., 1S32(?), p. 49, pl. 10, figs. 1, 2 (non Say, 1822). Gould, Inv. Mass., 1870, p. 62, fig. 379 .

ME. to Conn.- Common from low-water mark to 30 fathoms.

Pandora (Clidiophora) inornata (Verrill and Bush).

Clidiophora inornata Verrill and Bush, Proc. U. S. Nat. Mus., 1898, vol. 20, p. 819, pl. 95, figs. 5, 6. [off Massachusetts.]

Mass.-Off Stellwagen Bank, north of Cape Cod, and off Chatham, 10 to 43 fathoms.

\section{LYYNSIIDAE.}

\section{*Iyonsia hyalina (Conrad).}

Conrad, Amer. Marine Conch., 1832(?), p. 51, pl. 11, fig. 2. Gould, Inv. Mass., 1870, p. 64, fig. 380.

Mya hyalina Conrad, Journ. Acad. Nat. Sci. Phila., 1S31, ser. 1, vol. 6, p. 261, pl. 11, fig. 12. " NORTHERn COAST of THE U. S."

Osteodesma hyalina Couthouy, Boston Journ. Nat. Hist., 1839, vol. 2, p. 166 .

Me. to Conn.- Common from low-water mark to 30 fathoms.

*Lyonsia arenosa (Möller).

Gould, Inv. Mass., 1870, p. 65, fig. 381.

Pandorina arenosa Möller, Krøyer's Naturh. Tidskr., 1842, vol. 4, p. 93. [GREENLAND.]

ME.-Casco Bay; Frenchman's Bay; Eastport.

\section{POROMYACIDAE.}

Poromya granulata (Nyst and Westendorp).

Verrill, Trans. Conn. Acad., 1882, vol. 5, p. 564, pl. 44, figs. 3, 4. 
Corbula? granulata Nyst and Westendorp, Nouv. Rech. Coq. Foss. d'Anvers, 1839, p. 6, pl. 3, figs. 3, 4. [Belgium (fossil).]

Gulf of Maine, 150 fathoms.

Mass.- Off Marthas Vineyard, 63 to 146 fathoms.

\section{Poromya granulata rotundata Jefireys.}

Poromya rotundata Jeffreys, Ann. Mag. Nat. Hist., 1876, ser. 4, vol. 18, p. 494. [North Atlantic, 1450 fathoms.] Verrill, Proc. U. S. Nat. Mus., 1880, vol. 3, p. 396.

Mass.-South of Marthas Vineyard, 65 to 115 fathoms.

\section{VERTICORDIIDAE:}

Verticordia (Trigonulina) ornata caelata Verrill.

Verticordia caclata Verrill, Trans. Conn. Acad., 1882, vol. 5, p. 566. “off Martha's Vineyard." Verrill, Trans. Conn. Acad., 1884, vol. 6 , p. 278 , pl. 30 , figs. $9,9 a$.

Verticordia (Trigonulina) ornata Dall, Bull. Mus. Comp. Zoöl., 1886, vol. 12, p. 290.

Mass.-Off Marthas Vineyard, 100 fathoms.

Halicardia flexuosa (Verrill and Smith).

Dall, Proc. U. S. Nat. Mus., 1895, vol. 17, p. 697, pl. 23, figs. 1, 3, 5,6 ; pl. 24, fig. 3 .

Mytilimeria flexuosa Verrill and Smith in Verrill, Amer. Journ. Sci., 1881, ser. 3, vol. 22, p. 302. [off Marthas Vineyard.] Verrill, Trans. Conn. Acad., 1882, vol. 5, p. 567, pl. 58, fig. 38.

MAss.-Off Marthas Vineyard, 75 to 349 fathoms.

Lyonsiella abyssicola (M. Sars) G. O. Sars.

Verrill, Proc. U. S. Nat. Mus., 1880, vol. 3, p. 396.

Pecchiolia abyssicola G. O. Sars, Remark. Forms Anim. Life, 
1872, vol. 1, p. 25, pl. 3, figs. 21-43. [NEAR Lofoden IsLands, Norway.]

Mass.- South of Marthas Vineyard, 192 to 500 fathoms.

Lyonsiella insculpta (Jeffreys).

Pecchiolia insculpta Jeffreys, Proc. Zool. Soc. London, 1881, p. 932, pl. 70, fig. 4. "Bay of Biscay *** Palermo."

Lyonsiella gemma Verrill, Proc. U. S. Nat. Mus., 1880, vol. 3, p. 396.

Mass.—Off Marthas Vineyard, 75 to 487 fathoms.

Dacrydium vitreum (Möller).

Verrill, Trans. Conn. Acad., 1882, vol. 5, p. 579, pl. 44, figs. 8, 8 a.

Modiola? vitrea Möller, Krøyer's Naturh. Tidskr., 1842, vol. 4, p. 92. [GREENLAND.]

Gulf of Maine, 60 to 160 fathoms.

Mass.-Off Cape Cod, 106 to 118 fathoms; off Marthas Vineyard, 312 to 500 fathoms.

\section{CUSPIDARIIDAE.}

*Cuspidaria obesa (Lovén).

Verrill and Bush, Proc. U. S. Nat. Mus., 1898, vol. 20, p. \$04, pl. 75 , fig. 7 .

Neaera obesa Lovén, Öfvers. Kongl. Vet.-Akad. Forh., 1846, vol. 3, p. 180. "Вон[us]."

Gulf of Maine, 52 to 92 fathoms.

Mass.-Off Cape Cod, 106 fathoms; off Marthas Vineyard, 192 to 500 fathoms.

Cuspidaria pellucida (Stimpson).

Verrill and Bush, Proc. U. S. Nat. Mus., 1898, vol. 20, p. 805, pl. 75 , fig. 8 ; pl. 76 , fig. 8 . 
Neaera pellucida Stimpson, Smithson. Contr. Knowl., 1853, vol. 6, art. 5, p. 21, pl. 1, fig. 13. " off Long Island, [New York]."

Me.-- Eastport Harbor; off Casco Bay, 50 to 95 fathoms.

Cuspidaria glacialis (G. O. Sars).

Verrill and Bush, Proc. U. S. Nat. Mus., 1898, vol. 20, p. 800 , pl. 71, fig. 9; pl. 73, fig. 5; pl. 75, fig. 9 .

Neaera glacialis G. O. Sars, Moll. Reg. Arct. Norv., 1878, p. 88, pl. 6, fig. 8. [off Vads $\phi$, Norway.] Verrill, 'Trans. Conn. Acad., 1882, vol. 5, p. 562, pl. 44, figs. $10 a, 10 b$.

ME.-Off Casco Bay and in the Gulf of Maine, 50 to 180 fathoms.

Mass.-Off Cape Ann and Cape Cod; south of Marthas Vineyard, 65 to 500 fathoms.

\section{Cuspidaria rostrata (Spengler).}

Mya rostrate Spengler, Skriv. Nat.-Selsk. Kjobenhavn, 1793, vol. 3, pt. 1, p. 42. [Bergen, Norway.]

Neaera rostrata Verrill, Trans. Conn. Acad., 1882, vol. 5, p. 562, pl. 58 , fig. 39.

. Mass.- South of Marthas Vineyard, 85 to 155 fathoms.

Cuspidaria media Verrill and Bush.

Verrill and Bush, Proc. U. S. Nat. Mus., 1898, vol. 20, p. 800, pl. 71, figs. 5, 6; pl. 73, fig. 6. " off Martha's Vineyard."

Mass.-Common off Marthas Vineyard, 63 to 155 fathoms.

Cuspidaria (Cardiomya) multicostata Verrill and Smith.

Neaera multicostata Verrill and Smith, in Verrill, Proc. U. S. Nat. Mus., 1880, vol. 3, p. 398. "south of Martha's Vineyard."

Mass.- Off Marthas Vineyard, 85 to 158 fathoms. 
Cuspidaria (Cardiomya) perrostrata (Dall).

Neaera ornatissima d'Orbigny var. perrostrata Dall, Bull. Mus. Comp. Zoöl., 1881, vol. 9, p. 110. [off Tortugas.]

Cardiomya perrostrata Verrill and Bush, Proc. U. S. Nat. Mus. 1898, vol. 20, p. 809, pl. 73, fig. 2; pl. 74, fig. 3.

Mass.- Off Marthas Vineyard, 85 to 120 fathoms.

TELEODESMACEA.

PLEUROPHORIDAE.

*Cyprina islandica (Linné).

Lamarck, Hist. Nat. Anim. sans Vert., 1818, vol. 5, p. 557. Gould, Inv. Mass., 1870, p. 129, fig. 443.

Venus islandica Linné, Syst. Nat., ed. 12, 1767, vol. 1, p. 1131. "Istandia."

Me.-Casco Bay, 10 to 20 fathoms; Frenchman's Bay, 16 fathoms; Eastport.

Mass.-Massachusetts Bay; off Gay Head, 19 fathoms; Georges Bank, 45 fathoms.

R. I.-Off Block Island, 29 fathoms.

ASTARTIDAE.

*Astarte castanea (Say).

Gould, Inv. Mass., 1870, p. 117, fig. 431.

Venus castanea Say, Journ. Acad. Nat. Sci. Phila., 1822, ser. 1, vol. 2, p. 273. " COAST OF NEW JeRSEY."

Me.-Casco Bay; Frenchman's Bay, 25 to 30 fathoms.

MAss.-Massachusetts Bay; Chatham; Nantucket; Marthas Vineyard.

Conn.- Off New London. 


\section{${ }^{*}$ Astarte castanea picea Gould.}

Gould, Inv. Mass., 1841, p. 77. "Chelsea Beach, [Mass.]” Gould, Inv. Mass., 1870, p. 118.

Mass.- Revere; Vineyard Sound.

*Astarte castanea procera Totten.

Totten, Amer. Journ. Sci., 1835, ser. 1, vol. 28, p. 349, fig. 2. "Provincetown harbor, (Mass.)." Gould, Inv. Mass., 1870, p. 118.

Mass.-Provincetown; Marblehead; Nauset.

\section{*Astarte quadrans Gould.}

Gould, Inv. Mass., 1841, p. 81; fig. 48. “ From the Stomachs of fish caught in Massachusetts Bay."

ME.-Casco Bay; Frenchman's Bay.

MAss.-Massachusetts Bay; off Marthas Vineyard, 19 to 25 fathoms; off Provincetown, 20 fathoms; Georges Bank.

Conn.- Stonington (Linsley).

Astarte portlandica Mighels.

Mighels, Boston Journ. Nat. Hist., 1843, vol. 4, p. 320, pl. 16, fig. 2. "stomach of a haddock *** taken in Casco Bay." Blaney, Nautilus, 1906, vol. 19, p. 110.

ME.-Casco Bay; Frenchman's Bay, 10 fathoms (Blaney).

\section{*Astarte undata Gould.}

Gould, Inv. Mass., 1841, p. 80. [New England.]

Astarte sulcata Gould, Inv. Mass., 1870, p. 119, fig. 432 (non Da Costa).

Me.-Common, Casco Bay; Frenchman's Bay; Eastport.

N. H. 
MIAss. - Marblehead; Vineyard Sound and Buzzards Bay, 8 to. 25 fathoms; Duxbury.

R. I.- Off Block Island, 29 fathoms.

Conn.- Off New London and New Haven.

* Astarte undata latisulca (Hanley).

Dall, Proc. U. S. Nat. Mus., 1903, vol. 26, p. 938.

Crassina latisulca Hanley, Cat. Recent Biv. Shells, 1843, p. 87.

"N. America."

ME.-Frenchman's Bay, 16 to 30 fathoms; Eastport.

\section{*Astarte subaequilatera Sowerby.}

Sowerby, Thes. Conch., 1855, vol. 2, pt. 2, p. 780, pl. 167, fig. 13.

"Newfoundland."

Astarte crebricostata Gould, Inv. Mass., 1870, p. 126, fig. 440 (non Forbes).

Me.-Casco Bay, 25 fathoms; Penobscot Bay; Frenchman's Bay, 25 to 30 fathoms; Eastport, 14 fathoms.

MAss.- Off Nauset Light.

\section{Astarte subaequilatera whiteavesii Dall.}

Dall, Proc. U. S. Nat. Mus., 1903, vol. 26, pp. 939, 94̇, pl. 62, figs. 7, 12. "Gaspé ***200 FAthoms."

ME. to Conn.- Long Island Sound to the Gulf of St. Lawrence, 67 to 428 fathoms (Dall).

Astarte borealis (Schumacher).

Dall, Proc. U. S. Nat. Mus., 1903, vol. 26, p. 941.

Tridonta borealis Schumacher, Essai Nouv. Syst. Hab. Test., 1817, p. 147, pl. 17, fig. 1. No locality.

Astarte semisulcata Gould, Inv. Mass., 1870, p. 121, fig. 433.

ME.- Frenchman's Bay, 25 to 30 fathoms.

Mass. - Massachusetts Bay. 
Astarte elliptica (Brown).

Gould, Inv. Mass., 1870, p. 124, figs. 435-437.

Crassina elliptica Brown, Illustr. Conch., 1827, pl. 18, fig. 3. "Firth of Forth and Greenock."

Me. to Mass. - Massachusetts Bay, northward in 8 to 90 fathoms (Dall).

Astarte striata (Leach).

Nicania striata Leach, Ross's Voyage, 1819, Appendix no. 2, p. Ixii. " "LAT. $76^{\circ} 42^{\prime}$ N., LONG. $76^{\circ}$ W."

Astarte banksii Gould, Inv. Mass., 1870, p. 125, fig. 438 (non Leach).

Me.-Frenchman's Bay, 20 fathoms (Blaney).

Mass.- Massachusetts Bay northward, 10 to 85 fathoms (Dall).

\section{CRASSATELLITIDAE.}

*Crassinella mactracea (Linsley).

Astarte mactracea Linsley, Amer. Journ. Sci., 1845, ser. 1, vol. 48, p. 275, fig. "Stonington, [Conn.]". Gould, Amer. Journ. Sci., 1848, ser. 2, vol. 6 , p. 233, figs. $1,2$.

Gouldia mactracea Gould, Inv. Mass., 1870, p. 128, fig. 442.

Mass.- Vineyard Sound and Buzzards Bay, 3 to 10 fathoms.

Conn.- Stonington, codfish stomach (Linsley); off New London.

\section{SPHAERIIDAE.}

*Sphaerium sulcatum (Lamarck).

Prime, Smithson. Misc. Coll., 1865, vol. 7, art. 5, p. 33, fig. 25.

Cyclas sulcata Lamarck, Hist. Nat. Anim. sans Vert., 1818, vol. 5, p. 560. " lac Georges, Amérique septentrionale."

Sphaerium simile Gould, Inv. Mass., 1870, p. 101, fig. 407 (non Say). 
Me.- Barren Brook; Caribou; North Warren; Saco.

N. H.

Vт.-Chimney Point, Lake Champlain.

Mass.-Cambridge; Malden; Pontoosuc Lake; Blackstone River, Uxbridge.

R. I.- Ten Mile and Blackstone Rivers.

Conn.-Deep River; Housatonic River; Weston.

*Sphaerium striatinum (Lamarck).

Prime, Smithson. Misc. Coll., 1865, vol. 7, art. 5, p. 37, fig. 29.

Cyclas striatina Lamarck, Hist. Nat. Anim. sans Vert., 1818, vol. 5, p. 560. " L'AMÉRIQUE SEPTENTTIONALE."

Me.-Fish River; St. Johns River at Fort Kent (Nylander).

N. H.- Connecticut River, at Cornish.

Vт.- Hartland; Larrabee's Point.

Mass.-Connecticut River, at Sunderland; Blackstone River, Uxbridge.

Conn.- Housatonic River.

\section{Sphaerium stamineum (Conrad).}

Prime, Smithson. Misc. Coll., 1865, vol. 7, art. 5, p. 38, fig. 30.

Cyclas staminea Conrad, Amer. Journ. Sci., 1834, ser. 1, vol. 25, p. 342, pl. 1, fig. 5. " South Alabama."

Cons.

\section{Sphaerium solidulum (Prime).}

Prime, Smithson. Misc. Coll., 1865, vol. 7, art. 5, p. 36, fig. 27. Cyclas solidula Prime, Proc. Boston Soc. Nat. Hist., 1852, vol. 4, p. 158. "Онго."

Conn.

\section{*Sphaerium vermontanum Prime.}

Sphaerium vermontana Prime, Proc. Acad. Nat. Sci. Phila., 1861, p. 128. "Vermont." Gould, Inv. Mass., 1870, p. 105, fig. 410. 
VT.-Chimney Point, Lake Champlain; Lake Memphremagog. Mass.-Pontoosuc Lake, Berkshire Co.

\section{*Sphaerium emarginatum (Prime).}

Prime, Smithson. Misc. Coll., 1865, vol. 7, art. 5, p. 43, fig. 38.

Cyclas emarginata Prime, Proc. Boston Soc. Nat. Hist., 1852, vol. 4, p. 156. "Lake SUPerior."

ME.- Eagle Lake.

\section{*Sphaerium rhomboidum (Say).}

Cyclas rhomboida Say, Journ. Acad. Nat. Sci. Phila., 1822, ser. 1, vol. 2, p. 380. "Lake Champlain."

Cyclas elegans C. B. Adams, Boston Journ. Nat. Hist., 1840, vol. 3, p. 330, pl. 3, fig. 11 .

Sphaerium rhomboideum Prime, Smithson. Misc. Coll., 1865, vol. 7, art. 5, p. 39, fig. 31. Gould, Inv. Mass., 1870, p. 104, fig. 409.

ME.- Gelot Lake, New Sweden; Little Madawaska River.

VT.-Chimney Point, Lake Champlain.

Mass.-Cambridge; Readville; Newton Upper Falls; Housatonic River, Lenox.

R. I.- Pawtucket.

Conn.- Housatonic River.

\section{Sphaerium fabale (Prime).}

Cyclas fabalis Prime, Proc. Boston Soc. Nat. Hist., 1852, vol. 4, p. 159. "LAKe Superior."

Sphaerium fabalis Prime, Smithson. Misc. Coll., 1865, vol. 7, art. 5 , p. 40 , fig. 33 .

VT.

Conn.

\section{*Sphaerium occidentale (Prime).}

Prime, Smithson. Misc. Coll., 1865, vol. 7, art. 5, p. 41, fig. 34. Gould, Inv. Mass., 1870, p. 108, fig. 414. 
Cyclas ovalis Prime, Proc. Boston Soc. Nat. Hist., 1853, vol. 4, p. 276 (non Férussac, 1807). "Oswego and Greenwich, N. Y. *** Colunibus, O."

N. H. - Keene.

VT.-Hartland.

\section{*Musculium partumeium (Say).}

Cyclas partumeia Say, Journ. Acad. Nat. Sci. Phila., 1822, ser. 1, vol. 2, p. 380. "Near Germantown, [PA.]."

Cyclas orbicularia Linsley, Amer. Journ. Sci., 1845, ser. 1, vol.48, p. 276.

Cyclas coerulea Prime, Proc. Boston Soc. Nat. Hist., 1852, vol. 4, p. 161.

Sphaerium partumeium Gould, Inv. Mass., 1870, p. 103, fig. 408.

ME.-Great Pond, Cape Elizabeth; South Newcastle; North Haven.

N. H.- Keene.

Mass.-Cambridge; Readville; Revere.

R. I.-Bailey's Pond, Newport; Nyatt.

Conn.- Old Mill Hill Brook, Stratford; East Haddam.

* Musculium securis (Prime).

Cyclas securis Prime, Proc. Boston Soc. Nat. Hist., 1852, vol. 4, p. 160. "Massachusetts."

Sphaerium securis Gould, Inv. Mass., 1870, p. 107, fig. 413.

ME.-Aroostook River, Caribou; Scarboro; Saco; Old Orchard. VT. - Larrabee's Point.

Mass.-Cambridge; Neponset River, Green Lodge; Concord; East Wareham.

R. I.-Pawtucket River, below Pontiac; Bailey's Pond, Newport.

*Musculium securis cardissum (Prime).

Cyclas cardissa Prime, Proc. Boston Soc. Nat. Hist., 1852, vol. 4, 
p. 160. "Massachusetts*** in Fresh Pond, near CanBRIDGE, AND AT SALEMr."

ME.-Square Lake Inlet; Pout Pond, Westbrook.

Mass.-Readville; Charles River, Auburndale.

*Musculium deforme (H. F. Carpenter).

Sphaerium deformis Carpenter, Nautilus, 1902, vol. 16, p. 18. "Coventry, R. I."

Mass.-Agawam River, East Wareham.

R. I.- Tiogue Reservoir, Coventry.

Musculium truncatum (Linsley).

Cyclas truncata Linsley, Amer. Journ. Sci., 1845, ser. 1, vol. 48, p. 276. "Stonington, Stratford, and Stratfield [, Conn.]." Gould, Amer. Journ. Sci., 1848, ser. 2, vol. 6, p. 234, fig. 3.

Cyclas calyculata C. B. Adams, Amer. Journ. Sci., 1841, ser. 1, vol. 40, p. 277 (non Draparnaud).

Cyclas pellucida Prime, Proc. Boston Soc. Nat. Hist., 1853, vol. 4, p. 277.

Sphaerium truncatum Gould, Inv. Mass., 1870, p. 106, fig. 411.

ME.-Aroostook River.

VT.-Chimney Point, Lake Champlain.

Mass.

R. I.- Nayatt.

Conn.-Stratford; Stratfield; Stonington.

\section{Musculium tenue (Prime).}

Cyclas tenuis Prime, Proc. Boston Soc. Nat. Hist., 1852, vol. 4, p. 161. "Massachusetts *** New Bedford."

Sphaerium tenue Prime, Smithson. Misc. Coll., 1865, vol. 7, art. 5, p. 47, fig. 44. Gould, Inv. Mass., 1870, p. 107, fig. 412.

Me.-Androscoggin River.

VT.-Chimney Point, Lake Champlain. 


\section{Musculium transversum (Say).}

Cyclas transversa Say, New Harmony (Ind.) Disseminator, 1829, vol. 2, p. 356. [Nовтн Кемтuску.]

Sphaerium transversum Prime, Smithson. Misc. Coll., 1865, vol. 7, art. 5, p. 48 , fig. 45 .

ME.-Stroudwater (Norton).

Vт.-Chimney Point, Lake Champlain.

\section{Musculium winkleyi Sterki.}

Sterki, Nautilus, 1909, vol. 23, p. 66. “Old Orchard, ME.; vicinity of Danvers, Mass."

Me.-Old Orchard; Avon.

Mass.-Danvers; Cambridge; Woburn; Duxbury.

Musculium rosaceum (Prime).

Cyclas rosacea Prime, Proc. Boston Soc. Nat. Hist., 1852, vol. 4, p. 155. "Pennsylvania *** in the Schuylkill."

Sphaerium rosaceum Prime, Smithson. Mise. Coll., 1865, vol. 7, art. 5 , p. 50, fig. 48 .

Me.- Sandy River, Franklin Co.; Georges River, North Warren. Mass.-Charles River, Auburndale; Milton.

\section{*Musculium rykoltii (Normand).}

Cyclas rykoltii Normand, Notes sur Quelques Nouvelles Cyclades, 1844, p. 7, figs. 5, 6. [France.]

Calyculina rykoltii Clessin, Martini and Chemnitz's Conch. Cab., 1879, vol. 9, Monogr. Cycladeen, p. 257, pl. 40, figs. 20-27.

ME.--Warren; Thomaston.

\section{Musculium parvum Sterki.}

Sterki, Nautilus, 1909, vol. 23, p. 67. “Оніо; A SwaMp NeAr Uhrichsville; also in Stark and Summit Counties."

ME.-Georges River, North Warren. 


\section{*Pisidium abditum Haldeman.}

Haldeman, Proc. Acad. Nat. Sci. Phila., 1841, vol. 1, p. 53. "Lancaster Co. Pennsylvania." Gould', Inv. Mass., 1870, p. 113, figs. 425,426 .

Cyclas minor C. B. Adams, Proc. Boston Soc. Nat. Hist., 1844, vol. 1 , p. 48 .

ME.-Woodland; Bethel; Dennysville; Saco; Old Orchard; Ft. Fairfield.

N. H.- Keene.

VT.- Weybridge.

Mass-Cambridge; Stow; Revere; Danvers; Dalton; Duxbury.

R. I.

CONN.

\section{Pisidium inornatum Sterki.}

Sterki, Nautilus, 1911, vol. 25, p.3. "swAMPy LAND, ROANOKE, Randolph Co., Ala."

Mass.- Duxbury.

\section{*Pisidium aequilaterale (Prime).}

Prime, Boston Journ. Nat. Hist., 1852, vol. 6, p. 366, pl. 12, figs. 23-25. "Augusta, ME." Prime, Smithson. Misc. Coll., 1865 , vol. 7 , art. 5, p. 63 , figs. 65,66 .

Me.-Portage Lake; Little Madawaska River; Sebec Lake; Orono; Saco.

N. H.- Keene.

MAss.- Winchester; Waltham; Newton Upper Falls; Danvers. CONN.

\section{*Pisidium ferrugineum Prime.}

Prime, Proc. Boston Soc. Nat. Hist., 1852, vol. 4, p. 162. "SALem *** Cambridge, [Massachusetts]." Prime, Smithson. Misc. Coll., 1865, vol. 7 , art. 5 , p. 71 , figs. $77,78$. 
Me.-Fogelin's Lake, New Sweden; Jefferson; Saco; Portage Lake.

Mass.-Waltham; Newton Upper Falls; Danvers; Wareham; Duxbury.

\section{*Pisidium virginicum (Gmelin).}

Prime, Smithson. Misc. Coll., 1865, vol. 7, art. 5, p. 61, figs. 61, 62.

Tellina virginica Gmelin, Syst. Nat., 1790, vol. 1, pt. 6, p. 3236. " in Virginiae fluviss."

Cyclas dubia Say, Nich. Encycl., 1817, pl. 1, fig. 10.

Pisidium dubium Gould, Inv. Mass., 1870, p. 109, figs. 415, 416.

ME.-Saco.

N. H.- Connecticut River at Cornish.

VT.- Hartland.

Mass.-Connecticut River.

Cons.-- Housatonic River.

\section{*Pisidium adamsii Prime.}

Prime, in Stimpson, Shells of New England, 1S51, p. 16; Smithson. Misc. Coll., 1865, vol. 7, art. 5, p. 63, fig. 63.

Cyclas nitida Mighels and Adams, Boston Journ. Nat. Hist., 1842, vol. 4, p. 39, pl. 4, fig. 3 (non Jenyns). "Norway, Oxford Co., ME."

ME.-Caribou; Cross Lake Inlet; Saco; Westmanland.

Pisidium minus Stimpson.

Cyclas minor Mighels and Adams, Boston Journ. Nat. Hist., 1842, vol. 4, p. 39, pl. 4, fig. 2. "Weybridge, Vт."

Pisidium minus Stimpson, Shells of New England, 1S51, p. 16.

ME.-Black River, Warren; Mill River; Thomaston; Dennisville.

VT. - Weybridge.

Mass.-Duxbury; Woburn. 
Pisidium monas Sterki.

Sterki, Nautilus, 1901, vol. 14, p. 100. “Mountain Lake, Marquette County, Michigan."

Me.-Georges River, North Warren.

Pisidium alleni Sterki.

Sterki, Nautilus, 1912, vol. 26, p. 9. "New England, New York."

ME.-Hebron and Buckfield; Franklin Co.

N. H.

Pisidium sphaericum Sterki.

Sterki, Nautilus, 1912, vol. 26, p. 8. [Westbrook, Me., SACO, Me., and Lynnfield, Mass.]

Me.-Westbrook; Georges River, North Warren; Saco.

Mass.-Danvers; Lynnfield; Cambridge; Woburn.

*Pisidium variabile Prime.

Prime, Proc. Boston Soc. Nat. Hist., 1852, vol. 4, p. 163. "Massachusetrs." Prime, Smithson. Misc. Coll., 1865, vol. 7, art. 5, p. 66, figs. 69, 70. Gould, Inv. Mass., 1870, p. 115, figs. 427, 428.

Me.-Caribou; Mud Lake, Westmanland, Aroostook Co.; Saco; Dover; Ft. Fairfield.

N. H.

Mass.-Cambridge; Concord; Rowley; Waltham; Danvers; Duxbury; Wareham.

Pisidium variabile cicer Prime.

Pisidium cicer Prime, Ann. Lyc. Nat. Hist. N. Y., 1854, rol. 6, p. 64, pl. 1, fig. 1. "Washington County, N. Y."

ME.-Georges River, North Warren. 


\section{*Pisidium ventricosum Prime.}

Prime, Proc. Boston Soc. Nat. Hist., 1851, vol. 4, p. 68. "MasSachusetts ***Fresh Pond, Cambridge." Prime, Smithson. Misc. Coll., 1865, vol. 7, art. 5, p. 72, figs. 79, 80.

Me.- Barren Brook, Caribou; Ft. Fairfield; Westmanland.

Mass.-- Fresh Pond, Cambridge; Waltham; Danvers.

\section{*Pisidium rotundatum Prime.}

Prime, Proc. Boston Soc. Nat. Hist., 1852, vol. 4, p. 164. " LAKE Superior." Prime, Smithson. Misc. Coll., 1865, vol. 7, art. 5, p. 72 , figs. 81,82 .

ME.- Barren Brook, Woodland, Aroostook Co.; Rockland.

VT.-Chimney Point, Lake Champlain.

\section{*Pisidium compressum Prime.}

Prime, Proc. Boston Soc. Nat. Hist., 1852, vol. 4, p. 164. " MasSachusetts, ** Cambridge." Prime, Smithson. Misc. Coll., 1865, vol. 7, art. 5, p. 64, figs. 67,68 .

Me.--Barren Brook, Caribou; Cross Lake, Woodland.

N. H.-Keene.

VT.-Hartland; Chimney Point, Lake Champlain.

Mass.-Winchester; Woburn; Concord; Dalton; Danvers.

Conn.- Stonington.

\section{*Pisidium contortum Prime.}

Prime, Ann. Lyc. Nat. Hist. N. Y., 1854, vol. 6, p. 65, pl. 1, fig. 2. "Pittsfield, Mass.," (Post-Pliocene).

ME.-Caribou; Mud Lake; Perham and Westmoreland, Aroostook Co. (Nylander).

*Pisidium mainense Sterki.

Sterki, Nautilus, 1903, vol. 17, p. 21. 
Pisidium walkeri var. mainense Sterki, Nautilus, 1898, vol. 12, p. 79. "Caribou, Aroostook Co., Maine."

Me.- Hacket's Mill Brook; Woodland; Barren Brook, Caribou; Westmanland.

\section{Pisidum fallax Sterki.}

Sterki, Nautilus, 1896, vol. 10, p. 20. “Онго.”

ME.-Aroostook River.

Mass.-Groton.

\section{*Pisidium fallax boreale Sterki.}

Pisidium fallax var. sep[t]entrionale Sterki, Nautilus, 1898, vol. 12, p. 78 (non Prime, 1895). [Minnesota AND Aroostook Co., Maine.]

Pisidium fallax var. boreale Sterki, Nautilus, 1899, vol. 13, p. 12. Pisidium fallax var. errans Sterki, Nautilus, 1905, vol. 19, p. 84.

Me.- Little Madawaska River at New Sweden, and Aroostook River at Caribou (Nylander).

\section{*Pisidium pauperculum Sterki.}

Sterki, Nautilus, 1896, vol. 10, p. 64. [Massachusetts to Minnesota.]

Me.-Caribou; Little Madawaska River, New Sweden; Westmanland.

Mass.- Winchester; Newton Upper Falls; Groton.

\section{*Pisidium pauperculum nylanderi Sterki.}

Pisidium pauperculum var. nylanderi Sterki, Nautilus, 1898, vol. 11, p. 125. "Maine and New Jersey."

ME.- Caribou Stream; Portage, Long, and Square Lakes, Aroostook Co. 


\section{*Pisidium milium Held.}

Held, Oken's Isis, 1836, vol. 4, p. . [EuRopf.] Sterki, Nautilus, 1899, vol. 13, p. 10. Nylander, Nautilus, 1899, vol. 13, p. 59.

ME. - South branch of Caribou Stream in Woodland; Gelot's. Lake, New Sweden (Nylander).

*Pisidium medianum minutum Sterki.

Sterki, Nautilus, 1899, vol. 13, p. 113. [MaINE, NEW York, Michigan.]

Me.- Lovely Brcok Bog, Ft. Fairfield; Hacket's Mill Brook, Woodland.

Mass.-Danvers.

\section{*Pisidium splendidulum Sterki.}

Sterki, Nautilus, 1898, vol. 11, p. 113. [Maine, Michigan, And WASHington, D. C.]

Me.- Barren Brook, Caribou; Ft. Fairfield; Warren; Saco; Old Orchard.

Mass.-Danvers: Woburn.

R. I.--Bailey's Pond, Newport; Nyatt.

\section{*Pisidium roperi Sterki.}

Sterki, Nautilus, 1898, vol. 12, p. 77. "Maine, Rhode Istand, Indiana, Illinois, and Minnesota."

Me.-Johnson's Brook, Ft. Kent Road (Nylander); Denny:ville; Perham.

VT.-Chimney Point, Lake Champlain.

Mass.-Woburn.

R. I. 
*Pisidium politum Sterki.

Sterki, Nautilus, 1895, vol. 9, p. 75. [Oнio, Penna., Mich., ILL., AND Minn.]

Me.- Portage and Cross Lakes, Aroostook Co. (Nylander).

*Pisidium punctatum Sterki.

Sterki, Nautilus, 1895, vol. 8, p. 99, pl. 11, figs. 7-12. [STreams of Portage Co., Ohio.]

ME.- Portage Lake, Little Madawaska (Nylander).

Mass.- Wareham.

Pisidium punctatum simplex Sterki.

Sterki, Nautilus, 1905, vol. 19, p. 84. [Illinors; Michigan; Wisconsin.]

Me--Caribou Stream, Woodland; Georges River, North Warren.

Pisidium regulare Prime.

Prime, Boston Journ. Nat. Hist., 1852, vol. 6, p. 363, pl. 12, figs. 11-13. "Miami Canal, near Cincrnnati, Ohio."

Mass.- Concord.

\section{*Pisidium trapezoideum Sterki.}

Sterki, Nautilus, 1896, vol. 9, p. 124. [CAnadA, Pennsylvania, New Jersey (non Michigan, Minnesota and Texas, Sterki).]

Mass.- Cambridge (Roper Collection).

*Pisidium affine Sterki.

Sterki, Nautilus, 1901, vol. 15, p. 66. [Great Lakes Region, New York to Minnesota.] 
Me.- Mud Lake, Westmanland.

MAss.-Newton Upper Falls; Woburn (Winkley).

\section{Pisidium neglectum Sterki.}

Sterki, Nautilus, 1906, vol. 20, p. 87. " Krumroy, Summit Co., Онго."

Mass.-Danvers (Winkley).

\section{Pisidium succineum Sterki.}

Sterki, Nautilus, 1907, vol. 20, p. 99. [New York, Оніо, Michigan, Indiana, Virginia.]

ME.- Thomaston.

VT.-Chimney Point, Lake Champlain.

Mass.-Danvers (Winkley); Duxbury.

Pisidium henslowanum (Sheppard).

Tellina henslowana Sheppard, Trans. Linn. Soc. London, Zool., 1823, vol. 14, p. 150. [SUFFolK, ENGLAND.]

Pisidium henslowianum Forbes and Hanley, Brit. Moll., 1853, vol. 2, p. 131, pl. 37, fig. 11.

Corncocyclas (Tropidocyclas) henslowiana Dall, Harriman Alaska Exped., 1905, vol. 13, p. 145.

$\mathrm{ME}$.- Aroostook Co.

VT.-Chimney Point, Lake Champlain.

\section{${ }^{*}$ Pisidium walkeri Sterki.}

Sterki, Nautilus, 1895, vol. 9, p. 75. [Michigan, New York, Pennsylvania, Minnesota.]

Me.- Barren Brook, Caribou; Hacket's Mill Brook (Nylander).

*Pisidium subrotundum Sterki.

Sterki, Nautilus, 1906, vol. 20, p. 19. [New England, ANTIcosti Island, to Michigan.] 
ME.- Little Mud Lake, Aroostook Co.; Westmanland (Nylander).

MAss.-Cambridge; Woburn; Duxbury; Wareham; Westfield.

\section{*Pisidium tenuissimum Sterki.}

Sterki, Nautilus, 1901, vol. 14, p. 99. "Michigan."

ME.-Little Mud Lake, Aroostook Co. (subfossil).

Pisidium noveboracense Prime.

Prime, Ann. Lyc. Nat. Hist. N. Y., 1853, vol. 6, p. 66, pl. 1, fig. 3. "Washington ***and Herkiner Counties, N. Y." Prime, Smithson. Misc. Coll., 1865, vol. 7, art. 5, p. 67, fig. 71.

ME.-Buckfield (J. A. Allen); Fogelin's Lake, Aroostook Co. (Nylander).

\section{*Pisidium streatori Sterki.}

Sterki, Nautilus, 1901, vol. 14, p. 100. "Canaseraga River, N. Y.; Garrettsville, Ohio."

Me.-Hebron (J. A. Allen).

\section{Pisidium imbecille Sterki.}

Sterki, Nautilus, 1900, vol. 14, p. 5. "Byer's Trout Pond, And Button Lake, Kent Co., Mich."

Me.-Aroostook Co. (Nylander).

\section{Pisidium vesiculare Sterki.}

Sterki, Nautilus, 1896, vol. 10, p. 21. “Grand Rapids, MichiGAN."

Me.-Aroostook Co. (Nylander). 


\section{Pisidium glabellum Sterki.}

Sterki, Nautilus, 1913, vol. 26, p. 137. “ Hess Lake, Newaygo Co., Мiсн."

ME.- Barren Brook, Aroostook Co.

Mass.-Auburndale; Wareham; Ferncroft.

Pisidium lermondi Sterki.

Sterki, Nautilus, 1913, vol. 26, p. 138. “Duck Pond, Warren, ME."

ME.-Duck Pond, Warren.

Mass.- Bourne.

* Pisidium scutellatum Sterki.

Sterki, Nautilus, 1896, vol. 10, p. 66. “Pine Lake, * * LaKe Michigan * * Minnesota."

ME.- Barren Brook, Caribou.

VT. - Lake Champlain.

*Pisidium costatum Sterki.

Sterki, Nautilus, 1903, vol. 17, p. 22. "Fossil in A MarL BeD at Monitor, Bay Co., Michigan."

ME.- Mud Lake, Westmanland (subfossil).

\section{CARDITIDAE.}

*Venericardia (Cyclocardia) borealis (Conrad).

Dall, Proc. Acad. Nat. Sci. Phila., 1902, vol. 54, p. 703.

Cardita borealis Conrad, Amer. Marine Conch., 1832, p. 39, pl. 8, fig. 1. "Massachusetts." Gould, Inv. Mass., 1870, p. 146 , fig. 455 .

Me.- Casco Bay, common; Frenchman's Bay; Eastport. N. H. 
Mass.- Vineyard Sound and Buzzards Bay, 10 to 25 fathoms.

R. I.-Off Block Island, 29 fathoms.

Conn._- Off New London.

*Venericardia (Cyclocardia) novangliae (Morse).

Actinobolus (Cyclocardia) novangliae Morse, First Ann. Rept. Peabody Acad. Sci., 1869, p. 76, figs. [New England.]

Cyclocardia novengliae Verrill, Rept. Inv. Vineyard Sound, 1873, p. 684 , pl. 29, fig. 215.

Me.-Casco Bay; Frenchman's Bay, 15 to 35 fathoms; Eastport.

Mass.-Off Gay Head, 10 to 25 fathoms; Duxbury.

Conn.- Off New London.

\section{LUCINIDAE.}

Myrtaea lens (Verrill and Smith).

Dall, Proc. U. S. Nat. Mus., 1901, vol. 23, p. 804.

Loripes lens Verrill and Smith in Verrill, Amer. Journ. Sci., 1880, ser. 3, vol. 20, p. 400. "off CaPE Cod."

Mass.-Off Cape Cod, 50 to 100 fathoms; south of Marthas Vineyard, 60 to 192 fathoms.

\section{*Phacoides (Lucinoma) filosus (Stimpson).}

Dall, Proc. U. S. Nat. Mus., 1901, vol, 23, p. 809, pl. 40, fig. 11. Lucina radula Gould, Inv. Mass., 1841, p. 69 (non Montagu).

Lucina filosa Stimpson, Shells of New England, 1851, p. 17. “ Near P[oin]t Shirley ***Phillips Beach [Mass.]."

Me.- Casco Bay; near Machias.

Mass.-Phillips Beach; Shirley Point, 6 fathoms; off Gay Head, 19 fathoms.

R. I:- Off Block Island, 29 fathoms; off Newport.

Cons.-Stonington. 
Phacoides (Lucinoma) blakeanus (Bush).

Dall, Proc. U. S. Nat. Mus., 1901, vol. 23, p. 809.

Lucina blakeana Bush, Bull. Mus. Comp. Zoöl., 1893, vol. 23, p. 231, pl. 2, figs. 16, 17. "off Cape Fear, N. C."

Mass.

*Divaricella quadrisulcata (d'Orbigny).

Dall, Proc. U. S. Nat. Mus., 1901, vol. 23, p. 815.

Lucina divaricata Gould, Inv. Mass., 1841, p. 70 (non Linné).

Lucina quadrisulcata d'Orbigny, Voy. Amér. Mér., Moll., 1846, vol. 5, p. 584. "ANTILles."

Lucina strigilla Stimpson, Shells of New England, 1851, p. 17.

Lucina dentata Gould, Inv. Mass., 1870, p. 99, fig. 405 (non Wood, 1815).

Mass.-Nahant; Chatham; Nantucket; Vineyard Sound, 6 to 14 fathoms.

DIPLODONTIDAE.

Diplodonta (Sphaerella) verrilli Dall.

Dall, Trans. Wagner Free Inst. Sci., 1900, vol. 3, pt. 5, p. 1180.

Diplodonta turgida Verrill and Smith in Verrill, Amer. Journ. Sci., 1881, ser. 3, vol. 22, p. 303 (non Conrad, 1848). Verrill, Trans. Conn. Acad., 1882, vol. 5, p. 569, pl. 58, fig. 42. [off Marthas VINEYARD.]

Mass.- Off Marthas Vineyard, 69 fathoms.

THYASIRIDAE

Thyasira insignis (Verrill and Bush).

Dall, Proc. U. S. Nat. Mus., 1901, vol. 23, p. 785.

Cryptodon sarsii Verrill, Proc. U. S.' Nat. Mus., 1880, vol. 3, p. 399 (non Philippi). 
Cryptodon insignis Verrill and Bush, Proc. U. S. Nat. Mus., 1898, vol. 20, p. 785, pl. 91, figs. 1, 2. “ BETWEEN N. LAT. $44^{\circ} 54^{\prime}$, W. LONG. $59^{\circ} 46^{\prime} 45^{\prime \prime}$, AND N. LAT. $42^{\circ} 19^{\prime}$, W. LONG. $69^{\circ} 47 \frac{1}{2}^{\prime}$, IN 65 то 471 FATHOMS."

Mass. - Off Cape Cod, N. lat. $42^{\circ} 19^{\prime}$, W. long. $69^{\circ} 47^{\prime}$, northward in 65 to 471 fathoms.

\section{*Thyasira gouldii (Philippi).}

Dall, Proc. U. S. Nat. Mus., 1901, vol. 23, p. 786.

Lucina flexuosa Gould, Inv. Mass., 1841, p. 71, fig. 52 (non Montagu, 1813). [Massachusetts Bay.]

Lucina gouldii Philippi, Zeitsch. für Malak., 1845, p. 75.

Cryptodon gouldii Gould, Inv. Mass., 1870, p. 100, fig. 406.

Me.-Casco Bay; Frenchman's Bay, 20 to 40 fathoms; Eastport.

Mass.-Massachusetts Bay; Wiscasset; Buzzards Bay, 6 fathoms; Duxbury.

R. I.- Off Block Island, 29 fathoms.

ConN.- Stonington.

Thyasira trisinuata (d'Orbigny).

Dall, Proc. U. S. Nat. Mus., 1901, vol. 23, p. 786.

Lucina trisinuata d'Orbigny, Moll. Cubana, Spanish ed., 1846, vol. 2, p. 300, pl. 27, figs. 46-49. [CuвA.]

Cryptodon obesus Verrill, Amer. Journ. Sci., 1872, ser. 3, vol. 3, p. 287 , pl. 7 , fig. 2.

Me.-Casco Bay; Wiscasset.

Mass.- Massachusetts Bay; off Marthas Vineyard, 19 fathoms.

R. I.-Off Block Island, 29 fathoms.

Thyasira plana (Verrill and Bush).

Dall, Proc. U. S. Nat. Mus., 1901, vol. 23, p. 786.

Cryptodon planus Verrill and Bush, Proc. U. S. Nat. Mus., 1898, vol. 20, p. 788, pl. 88, figs. 3, 4. “ NORTh of CAPE CoD," ETC. 
Me.-Casco and Frenchman's Bays; Wiscasset; Penobscot Bay.

Mass. - North of Cape Cod, 8 to 100 fathoms.

Thyasira inequalis (Verrill and Bush).

Cryptodon (Axinulus) inequalis Verrill and Bush, Proc. U. S. Nat. Mus., 1898, vol. 20, p. 791, pl. 90, figs. 1, 2 . “NORTH of Cape Cod," etc.

Thyasira inaequalis Dall, Proc. U. S. Nat. Mus., 1901, vol. 23, p. 786.

Me.- Casco Bay.

Mass.- North of Cape Cod, 14 to 49 fathoms.

Thyasira croulinensis (Jeffreys).

Clausina croulinensis Jeffreys, Ann., Mag. Nat. Hist., 1847, ser. 1, vol. 20, p. 19. "Croulin Island, Between Skye AND THE MAINLAND OF RossshiRe."

Cryptodon croulinensis Verrill and Bush, Proc. U. S. Nat. Mus., 1898, vol. 20, p. 786, pl. 90, figs. 3,4 .

Mass. - North of Cape Cod, 13 to 73 fathoms.

Thyasira equalis (Verrill and Bush).

Dall, Proc. U. S. Nat. Mus., 1901, vol. 23, p. 787.

C'ryptodon cqualis Verrill and Bush, Proc. U. S. Nat. Mus., 1898, vol. 20, p. 788, pl. 91, figs. 5, 6. “ BETWEEN N. LAT. $47^{\circ} 40^{\prime}$, W. LONG. $47^{\circ} 35^{\prime} 30^{\prime \prime}$, AND N. LAT. $37^{\circ} 08^{\prime}$, W. LONG. $74^{\circ} 33^{\prime} . "$

ME.- Eastport; Gulf of Maine.

Mass. - North of Cape Cod, 14 to 36 fathoms.

Thyasira (Axinulus) obsoleta (Verrill and Bush).

Cryptodon obsoletus Verrill and Bush, Proc. U. S. Nat. Mus., 1898, vol. 20, p. 789, pl. 89, figs. 1, 2. "off Marthas VineYARD."

Mass. - Off Marthas Vineyard, 100 to 390 fathoms. 
Thyasira (Axinulus) ferruginosa (Forbes).

Dall, Proc. U. S. Nat. Mus., 1901, vol. 23, p. 787.

Kellia ferruginosa Forbes, Rept. Brit. Assoc. Adv. Sci. for 1843, 1844, p. 192. [MoREA.]

Cryptodon (Aximulus) ferruginosus Verrill and Bush, Proc. U. S. Nat. Mus., 1898, vol. 20, p. 793, pl. 87, figs. 7, 8 .

Mass.-Off Marthas Vineyard, 153 to 500 fathoms.

Thyasira (Axinulus) ovata (Verrill and Bush).

Cryptodon (Axinulus) ovatus Verrill and Bush, Proc. U. S. Nat. Mus., 1898, vol. 20, p. 793, pl. 91, fig. 7; pl. 93, fig. 1. [oFf Marthas Vineyard.]

Mass.- South of Marthas Vineyard, 100 fathoms.

Thyasira (Axinulus) succisa (Jeffreys).

Dall, Proc. U. S. Nat. Mus., 1901, vol. 23, p. 788.

Axinus incrássatus var. succisa Jeffreys, Ann. Mag. Nat. Hist., 1876, ser. 4, vol. 18, p. 492. "North Atlantic and MediterRANEAN."

Off New England, 92 to 1366 fathoms.

Axinopsis orbiculata G. O. Sars.

G. O. Sars, Moll. Reg. Arct. Norv., 1878, p. 63, pl. 19, figs. 11a-d. “Vads $\varnothing . "$ Verrill, Trans. Conn. Acad., 1882, vol. 5, p. 569. Dall, Proc. U. S. Nat. Mus., 1901, vol. 23, p. 789.

Me.-Broad Sound, Casco Bay, 15 to 30 fathoms; Frenchman's Bay, 15 fathoms.

Axinopsis orbiculata inequalis Verrill and Bush.

Verrill and Bush, Proc. U. S. Nat. Mus., 1898, vol. 20, p. 794, pl. 92, figs. 5, 6. "BAY of Fundy."

ME.-Frenchman's Bay, 15 fathoms.

Mass.-Off Cape Ann, 18 to 26 fathoms. 
Axinopsis cordata Verrill and Bush.

Verrill and Bush, Proc. U. S. Nat. Mus., 1898, vol. 20, p. 795, pl. 97, figs. 5, 6. [off Marthas Vineyard.]

Mass.- Off Marthas Vineyard, 43 to 202 fathoms.

Leptaxinus minutus Verrill and Bush.

Verrill and Bush, Proc. U. S. Nat. Mus., 1898, vol. 20, p. 797, pl. 89, figs. 3-5. "N. LAT. $40^{\circ} 3^{\prime}$, W. LONG. $70^{\circ} 31^{\prime}$, IN 100 FATHOMS."

MAss.- Off Marthas Vineyard, 100 fathoms.

\section{LEPTONIDAE.}

\section{Kellia suborbicularis gouldi (Thomson).}

Dall, Proc. U. S. Nat. Mus., 1899, vol. 21, p. 889.

? Lepton fabagella Conrad, Amer. Marine Conch., 1831(?), p. 53, pl. 2 , fig. 3 .

Montacuta gouldi Thomson, Amer. Journ. Conch., 1567, vol. 3, p. 33, pl. 1, fig. 15. "N[Ew] BedFord harbor [MAss.]."

Kellia suborbicularis Gould, Inv. Mass., 1870, p. 83, fig. 394 (non Montagu).

Mass.-Off Salem; New Bedford Harbor.

R. I.- Narragansett Bay.

Rochefortia planulata (Stimpson).

Dall, Trans. Wagner Free Inst. Sci., 1909, vol. 3, pt. 5, p. 1161, pl. 45 , fig. 7 .

Kellia planulata Stimpson, Shells of New England, 1S51, p. 17. "New Bedford hare[or]," etc., Mass.

Kellia mbra Gould, Inv. Mass., 1841, p. 60, fig. 23 [= 33] (non Turton).

Montacuta bidentata Verrill and Bush, Proc. U. S. Nat. Mus. 1898, vol. 20, p. 779, pl. 93, figs. 7, 8; pl. 94, fig. 6 (non Montagn, non Gould); var. fragilis, p. 7SO pl. 92, fig. 8 . 
Me.- Casco Bay; Eastport, 8 to 15 fathoms.

Mass.-Boston Harbor, 5 fathoms; Provincetown; Vineyard Sound and Buzzards Bay, 1 to 8 fathoms; Duxbury.

R. I.-Off Block Island; Narragansett Bay; near Watch Hill. Conn.-- Near New Haven; Branford.

Rochefortia ovata (Jeffreys).

Montacuta ovata Jeffreys, Proc. Zool. Soc. London, 1881, p. 698, pl. 61, fig. 4. [off Spain.] Verrill and Bush, Proc. U. S. Nat. Mus., 1898, vol. 20, p. 781, pl. 92, figs. 9, 10.

Mysella ovata Dall, Proc. U. S. Nat. Mus., 1899, vol. 21, p. 890.

Mass. - Off Marthas Vineyard, 100 to 157 fathoms.

Montacuta percompressa Dall.

Dall, Proc. U. S. Nat. Mus., 1899, vol. 21, p. 894. "Woods Hole, [Mass.]."

Tellimya ferruginosa Verrill, Trans. Conn. Acad., 1884, vol. 6, p. 225, pl. 30, fig. 13 (non Montagu).

Mass.-At the "Gutters," Naushon Island, near Woods Hole.

KELLIELLIDAE.

*Turtonia minuta (Fabricius).

Gould, Inv. Mass., 1870, p. 85, fig. 395. Dall, Proc. U. S. Nat. Mus., 1899, vol. 21, p. 895.

Venus minuta Fabricius, Fauna Groenlandica, 1780, p. 412. [GREENLAND.]

ME.- Casco Bay; Frenchman's Bay.

Mass.-Massachusetts Bay; Duxbury.

*Aligena elevata (Stimpson).

Dall, Proc. U. S. Nat. Mus., 1899, vol. 21, p. 895.

Montacuta elevata Stimpson, Shells of New England, 1851, p. 16.

"New Bedford harb[or] *** Chelsea Beach, [Mass.]." 
Montacuta bidentata Gould, Inv. Mass., 1841, p. 59.

Kelliopsis elevata Verrill and Bush, Proc. U. S. Nat. Mus., 1898, vol. 20, p. 784, pl. 93, figs. 2-4; pl. 94, figs. 7, 8 .

Mass.-Revere; Wellfleet; Woods Hole; New Bedford Harbor; Duxbury.

R. I.- Narragansett Bay, $8 \frac{1}{2}$ to 10 fathoms.

Conn.- Savin Rock, near New Haven.

\section{CARDIIDAE.}

*Cardium (Cerastoderma) ciliatum Fabricius.

Cardium ciliatum Fabricius, Fauna Groenlandica, 1780, p. 410. [GREENLAND.]

Cardium pubescens Couthouy, Boston Jcurn. Nat. Hist., 1838, vol. 2, p. 61, pl. 3, fig. 6 .

Cardium islandicum Gould, Inv. Mass., 1870, p. 139, fig. 450 (non Linné).

Me. - Casco Bay; Frenchman's Bay, 20 to 30 fathoms; Eastport, 6 to 60 fatboms.

Mass. - Marblehead Harbor, 7 fathoms; off Cape Cod, 35 fathoms; Duxbury, 20 fathoms.

*Cardium (Cerastoderma) pinnulatum Conrad.

Cardium pinnulatum Conrad, Journ. Acad. Nat. Sci. Phila., 1831, ser. 1, vol. 6, p. 260, pl. 11, fig. 8. "Massachusetts." Gould, Inv. Mass., 1870, p. 141, fig. 452.

Me to Conn.- Common, especially north of Cape Cod.

*Cardium (Laevicardium) mortoni Conrad.

Cardium mortoni Conrad, Journ. Acad. Nat. Sci. Philə., 1831, ser. 1, vol. 6, p. 259, pl. 11, figs. 5-7. “ NORTHERn COAST [OF United States].”

Liocardium mortoni Gould, Inv. Mass., 1870, p. 143, fig. 453. 
Mass.- Cohasset; Vineyard Sound and Buzzards Bay; Nantasket; Duxbury.

R. I.-- Narragansett Bay; Buttonwoods; Wickford.

Conn.- New Haven.

\section{*Serripes groenlandicus (Gmelin).}

Cardium groenlandicum Gmelin, Syst. Nat., 1790, vol. 1, pt. 6, p. 3252. "Groenlandia et Islandia."

Aphrodite groenlandica Gould, Inv. Mass., 1870, p. 144, fig. 454. Serripes gronlandicus Dall, Proc. U. S. Nat. Mus., 1900, vol. 23, p. 388.

Me.-Casco Bay, 8 fathoms; Frenchman's Bay, 6 to 16 fathoms.

Mass.- Cape Cod Bay.

Conn.- Off Stonington (Linsley).

Protocardia peramabilis (Dall).

Dall, Proc. U. S. Nat. Mus., 1900, vol. 23, p. 388.

Cardium (Fulvia) peramabilis Dall, Bull. Mus. Comp. Zoöl., 1881, vol. 9, p. 132 . [West Indies.] Dall, Bull. Mus. Comp. Zoöl., 1886, vol. 12, p. 269, pl. 4, fig. 7 .

Off Rhode Island, 115 fathoms.

\section{VENERIDAE.}

*Callocardia (Agriopoma) morrhuana (Linsley).

Dall, Proc. U. S. Nat. Mus., 1902, vol. 26, p. 370.

Cytherea convexa Conrad, Journ. Acad. Nat. Sci. Phila., 1831, ser. 1, vol. 6, p. 261. Gould, Inv. Mass., 1870, p. 131, fig. 444 (non Say, 1824).

Cytherea morrhuana Linsley, Amer. Journ. Sci., 1845, ser. 1, vol. 48, p. 276. " CODfish stomach, Stonington, [Conn.]."

ME.-Casco Bay, 3 to 8 fathoms; Eastport. 
Mass.-Marblehead; Nahant; Provincetown; Vineyard Sound and Buzzards Bay, 2 to 10 fathoms.

R. I.- Narragansett Bay.

Conv.-Stonington; Stratford; New Haven.

*Venus mercenaria Linné.

Linné, Syst. Nat., ed. 10, 1758, vol. 1, p. 686. " Pensyluania." Gould, Inv. Mass., 1870, p. 133, fig. 445.

Me.-Quahog Bay, in Casco Bay.

Mass.-Revere; Provincetown; common south of Cape Cod.

R. I.- Narragansett Bay; Buttonwoods.

Conn.- Common along the entire coast.

*Venus mercenaria notata Say.

Venus notata Say, Journ. Acad. Nat. Sci. Phila., 1822, ser. 1, vol. 2, p. 271. " cOAst of the United States." Gould, Inv. Mass., 1870, p. 135, fig. 446.

Mass.- Edgartown.

R. I.- Newport; Buttonwoods.

Conn.- Stonington:

\section{Liocyma fluctuosa (Gould).}

Dall, Proc. U. S. Nat. Mus., 1902, vol. 26, p. 364.

Venus fluctuosa Gould, Inv. Mass., 1841, p. 87, fig. 50. "BANK FISHERIES."

Tapes fluctuosa Gould, Inv. Mass., 1870, p. 136, fig. 447.

ME.-Frenchman's Bay, 35 fathoms (Blaney).

\section{*Gemma gemma ('Totten).}

Gould, Inv. Mass., 1870, p. 137, fig. 448.

Venus gemma Totten, Amer. Journ. Sci., 1834, ser. 1, vol. 26, p. 367, pl. 1, fig. 2. "Massachusetts and Rhode Island." 
Gemma tottenii Stimpson, Smithson. Misc. Coll., 1860, vol. 2, art. 6 , no. 3 , p. 3 .

Totteniana gemma Perkins, Proc. Boston Soc. Nat. Hist., 1869, vol. 13 , p. 148.

ME. to Conn.- Especially abundant on the sandy shores of Mássachusetts.

*Gemma gemma purpurea (H. C. Lea).

Dall, Trans. Wagner Free Inst. Sci., 1903, iol. 3, pt. 6, p. 1332, pl. 24, figs. 2, 4, 4b.

Cyrena purpurea H. C. Lea, Amer. Journ. Sci., 1842, ser. 1, vol. 42, p. 106, pl. 1, fig. 1. "Delatware Bay."

Gemma manhattensis Gould, Inv. Mass., 1870, p. 138, fig. 449.

Mass.- Vineyard Sound.

R. I.- Buttonwoods.

Conn.- Near New Haven.

PETRICOLIDAE.

*Petricola pholadiformis Lamarck.

Lamarck, Hist. Nat. Anim. sans Vert., 1818, vol. 5, p. 505. Gould, Inv. Mass., 1870, p. 90, fig. 398.

Petricola fornicata Say, Journ. Acad. Nat. Sci. Phila., 1822, ser. 1, vol. 2, p. 319. " COAST OF North America."

Me.-Quahog Bay, in Casco Bay; Old Orchard Beach.

Mass.-Salem; Revere; Cohasset; Vineyard Sound and Buzzards Bay.

R. I.- Narragansett Bay.

Conn.- Near New Haven.

*Petricola dactylus Sowerby.

Sowverby, Genera Shells, 1820-1825, pl. 53, fig. 3; Thes. Conch., 1855, vol. 2, pt. 2, p. 772, pl. 166, fig. 4. "South AMERICA." 
Gastranella tumida Verrill, Amer. Journ. Sci., 1872, ser. 3, vol. 3, p. 286, pl. 6, figs. 3, 3a (young).

Me.- Casco Bay.

Mass.- Ipswich Beach; Lynn; Cohasset; Cataumet; Chatham.

Conn.- Long Island, near New Haven, 4 to 6 fathoms.

TELLINIDAE.

*Tellina (Angulus) tenera Say.

Tellina tenera Say, Journ. Acad. Nat. Sci. Phila., 1822, ser. 1, vol, 2, p. 303. " COASt of New Jersey." Gould, Inv. Mass., 1870, p. 97, fig. 403.

Anguhus tener Verrill, Rept. Inv. Vineyard Sound, 1873, p. 677, pl. 26, fig. 180; pl. 30, fig. 223.

Me.- Casco Bay.

Mass.- Ipswich Beach; Revere; Vineyard Sound and Buzzards Bay.

Conn.- Stonington; New Haven.

Tellina (Angulus) tenella (Verrill).

Angulus modestus Verrill, Amer. Journ. Sci., 1872, ser. 3, vol. 3, p. 285; pl. 6, figs. 2, 2a (non Carpenter, 1864). [VineYard Sound, Buzzards Bay and Long Island Sound.]

Angulus tenellus Verrill, Rept. Inv. Vineyard Sound, 1873, p. 677, pl. 30, fig. 224.

Mass.- Vineyard Sound and Buzzards Bay, 6 to 10 fathoms.

Conn.- Long Island Sound, off New Haven, 4 to 5 fathoms.

Tellina (Angulus) versicolor DeKay.

Tellina versicolor Cozzens, in Jay's Cat. Shells, ed. 2, 1836, p. 12. "New-York." DeKay, Nat. Hist. N. Y., Moll., 1843, p. 209, pl. 26, fig. 172 .

Conn.- Stratford; Long Beach. 
*Macoma balthica (Linné).

Tellina balthica Linné, Syst. Nat., ed. 10, 1758, vol. 1, p. 677. "M. Balthico."

Macoma fusca Gould, Inv. Mass., 1870, p. 93, fig. 400.

Macoma fragilis Verrill, Rept. Inv. Vineyard Sound, 1S73, p. 676, pl. 30, fig. 222.

ME. to Conn.- Common, especially in the harbors and bays.

* Macoma calcarea (Gmelin).

Tellina calcarea Gmelin, Syst. Nat., 1790, vol. 1, pt. 6, p. 3236. "MARI Islandiam Circumfluente."

Tellina sordida Couthouy, Boston Journ. Nat. Hist., 1838, vol. 2, p. 59, pl. 3, fig. 11 .

Macoma proxima Gould, Inv. Mass., 1870, p. 95, fig. 401.

Macoma sabulosa Verrill, Rept. Inv. Vineyard Sound, 1873, p. 677 .

ME.- Casco Bay, 3 to 60 fathoms; Frenchman's Bay, 10 to 15 fathoms; Eastport.

Mass.- Off Cape Ann; Georges Bank.

R. I.- Off Block Island, 29 fathoms.

Conn.- Stonington (Linsley).

*Macoma tenta (Say).

Tellina tenta Say, Amer. Conch., 1834, pl. 65, fig. 3. [South Carolina.] Gould, Inv. Mass., 1870, p. 96, fig. 402.

ME.-Georges River, near Taylor's Point, Cushing's Shore, 6 fathoms (Lermond).

Mass.-Vineyard Sound and Buzzards Bay, 2 to 10 fathoms; New Bedford.

Conn.- Stonington. 


\section{PSAMMODIIDAE.}

*Tagelus gibbus (Spengler).

Verrill, Rept. Inv. Vineyard Sound, 1873, p. 675, pl. 26, fig. 181; pl. 30, fig. 217.

Solen gibbus Spengler, Skriv. Nat.-Selsk. Kjobenhavn, 1794, vol. 3, pt. 2, p. 104. "I Vestindien."

Solecurtus gibbus Gould, Inv. Mass., 1870, p. 43, fig. 367.

Mass. - Vineyard Sound and Buzzards Bay; New Bedford; Chatham.

ConN.- Stratford (Linsley).

*Tagelus divisus (Spengler).

Verrill, Rept. Inv. Vineyard Sound, 1873, p. 676, pl. 30, fig. 218.

Solen divisus Spengler, Skriv. Nat.-Selsk. Kjobenhavn, 1794, vol. 3, pt. 2, p. 96. "Tunis."

Solecurtus divisus Gould, Inv. Mass., 1870, p. 44, fig. 368.

N. H.- Hampton Beach.

Mass.- Coffin's Beach; Hyannisport; Vineyard Sound and Buzzards Bay.

R. I.

Conn.-- Stonington.

\section{SEMELIDAE.}

* Cumingia tellinoides (Conrad).

Gould, Inv. Mass., 1870, p. 79, fig. 390.

Mactra tellinoides Conrad, Journ. Acad. Nat. Sci. Phila., 1831, ser. 1, vol. 6, p. 258, pl. 11, figs. 2, 3. "NORTHERN COAST [OF United States]."

Mass.-Provincetown; Vineyard Sound and. Buzzards Bay, 3 to 12 fathoms.

R. I.

Conn.- New Haven; off Stonington. 


\section{Abra aequalis (Say).}

Verrill, Rept. Inv. Vineyard Sound, 1873, p. 679.

Amphidesma aequalis Say, Journ. Acad. Nat. Sci. Phila., 1822, ser. 1, vol. 2, p. 307. " southern CoAst [of United States]."

ConN.- Stonington, from cod stomach (Linsley).

\section{SOLENIDAE.}

Solen viridis Say.

Say, Journ. Acad. Nat. Sci. Phila., 1822, ser. 1, vol. 2, p. 316. "southern coast [of United States]." Verrill, Rept. Inv. Vineyard Sound, 1873, p. 675.

R. I.

Conn.- Stonington (Linsley).

*Ensis directus (Conrad).

Dall, Proc. U. S. Nat. Mus., 1S99, vol. 22, p. 107.

Solen directus Conrad, Proc. Acad. Nat. Sci. Phila., 1843, vol. 1, p. 325. "Neuse River, Below Newbern, North Carolina. Mrocene?"

Solen ensis var. americanus Gould, Inv. Mass., 1870, p. 42, fig. 366 .

Ensatella americana Verrill, Rept. Inv. Vineyard Sound, 1873, p. 674, pl. 26, fig. 182 ; pl. 32 , fig. 245.

Me. to Conn.- The common "Razor Shell."

* Siliqua costata (Say).

Verrill, Rept. Inv. Vineyard Sound, 1873, p. 675, pl. 32, fig. 244.

Solen costatus Say, Journ. Acad. Nat. Sci. Phila., 1822, ser. 1, vol. 2, p. 315. "Great Egg Harbour, New Jersey."

Machaera costata Gould, Inv. Mass., 1870, p. 47, fig. 370.

ME.-Casco Bay; Scarboro.

Mass.-Revere; Nahant. 
R. I.

Conn.- New Haven.

Siliqua squama (Blainville).

Solecurtus squama Blainville, Dict. des Sci. Nat., 1827, vol. 49, p. 419. "Terre-Neuve."

Machaera squama Gould, Inv. Mass., 1870, p. 46, fig. 369.

Not unfrequently taken from the stomachs of codfish caught at the Banks and sometimes off our shores (Gould).

\section{MACTRIDAE.}

*Spisula (Hemimactra) solidissima (Dillwyn).

Dall, Nautilus, 1894, vol. 8, p. 26.

Mactra solidissima Dillwyn, Cat. Recent Shells, 1817, vol. 1, p. 140. “North America." Gould, Inv. Mass., 1870, p. 73, fig. 387.

Me. to Conn.- The Common Beach Clam or Hen Clam.

\section{Spisula (Hemimactra) solidissima similis (Say).}

Mactra similis Say, Journ. Acad. Nat. Sci. Phila., 1822, ser.1, vol. 2, p. 309. " COASt of the United States."

Mass.- Provincetown.

R. I.

Conn.

*Spisula (Hemimactra) polynyma (Stimpson).

Mactra polynyma Stimpson, Smithson. Misc. Coll., 1860, vol. 2, art. 6, no. 3, p. 3 .

Mactra ovalis Gould, Inv. Mass., 1841, p. 53, fig. 32 (non Sowerby, 1817). Gould, Inv. Mass., 1870, p. 75, fig. 388.

Me.-Casco Bay; Frenchman's Bay; Eastport; Penobscot Bay.

MAss.-Massachusetts Bay; Georges Bank. 
*Mulinia lateralis (Say).

Verrill, Rept. Inv. Vineyard Sound, 1873, p. 680, pl. 26, fig. 184, B (not fig. 185, B).

Mactra lateralis Say, Journ. Acad. Nat. Sci. Phila., 1822, ser. 1, vol. 2, p. 309. "COAST of The United States." Gould, Inv. Mass., 1870, p. 77, fig. 389.

ME.- Casco Bay.

Mass.-Lynn; Boston; Vineyard Sound and Buzzards Bay, 1 to 15 fathoms.

R. I.- Field's Point; Bristol.

Conn.- New Haven; Branford.

\section{MESODESMATIDAE.}

*Mesodesma arctatum (Conrad).

II actra arctata Conrad, Journ. Acad. Nat. Sci. Phila., 1831, ser. 1, vol. 6, p. 257, pl. 11, fig. 1. "Massachusetts."

Ceronia arctata Gould, Inv. Mass., 1870, p. 80, fig. 391.

ME.

Mass.-- Plum Island; Nahant; Truro; Chatham; Nantucket.

ConN.- Stonington.

Mesodesma deauratum (Turton).

Mactra deaurata Turton, Conch. Insul. Brit. Dithyra, 1S30, p. 71, pl. 5, fig. 5. "Exмотth [England]."

Ceronia deaurata Gould, Inv. Mass., 1870, p. 81, fig. 392.

Georges Bank (Gould).

MYACIDAE.

* Mya arenaria Linné.

Linné, Syst. Nat., ed. 10, 1758, vol. 1, p. 670. "O. EuropaE Septentrionalis.” Gould, Inv. Mass., 1870, p. 55, fig. 375.

ME, to Coxi. - The Common Clam of the New England coast. 
*Mya truncata Linné.

Linné, Syst. Nat., ed. 10, 1758, vol. 1, p. 670. “ O. Europaeo." Gould, Inv. Mass., 1870, p. 58, fig. 376.

Me.-Casco Bay; Frenchman's Bay; Eastport.

Mass.- Massachusetts Bay; Georges Bank.

\section{CORBULADAE.}

*Corbula contracta Say.

Say, Journ. Acad. Nat. Sci. Phila., 1822, ser. 1, vol. 2, p. 312. "coasts of Georgia and East Florida." Gould, Inv. Mass., 1870, p. 60 , fig. 377 .

Mass.- Vineyard Sound and Buzzards Bay, 5 to 19 fathoms.

R. I.- Narragansett Bay.

Conn.- Near New Haven.

\section{SAXICAVIDAE}

*Saxicava arctica (Linné).

Gould, Inv. Mass., 1870, p. 89.

Mya arctica Linné, Syst. Nat., ed. 12, 1767, p. 1113. “OCEANo Norvegrco."

Saxicava rugosa Lamarck, Hist. Nat. Anim. sans Vert., 1818, vol. 5, p. 501. Gould, Inv. Mass., 1870, p. 87, fig. 397.

Me. to Conn.-Commonly found adhering to other objects.

${ }^{*}$ Cyrtodaria siliqua (Spengler).

Mya siliqua Spengler, Skriv. Nat.-Selsk. Kjobenhavn, 1793, vol. 3, pt. 1, p. 48. "Newfoundland."

Glycymeris siliqua Gould, Inv. Mass., 1870, p. 53, fig. 374.

Me.-Casco Bay; Frenchman's Bay, 5 fathoms.

Mass.-Ipswich Bay; Nahant; off Provincetown; Buzzards Bay; Duxbury, 20 fathoms. Georges Bank. 
Panomya arctica (Lamarck).

Iredale, Proc. Mal. Soc. London, 1915, vol. 11, p. 341.

Glycimeris aretica Lamarck, Hist. Nat. Anim. sans Vert., 1818, vol. 5, p. 458. "L'Océan arctique, la Mer blanche."

Iya norregica Spengler, Skriv. Nat.-Selsk. Kjobenharn, 1793, vol. 3, pt. 1, p. 46, pl. 2, fig. 18 (non Gmelin, 1790).

Panopaea arctica Gould, Inv. Mass., 1870, p. 51, fig. 373.

Me.-Off Casco Bay, 115 fathoms; Frenchman's Bay, 25 to 30 fathoms; Eastport, 40 fathoms. Georges Bank.

\section{PHOLADIDAE.}

*Barnea costata (Linné).

Pholas costatus Linné, Syst. Nat., ed. 10, 1758, vol. 1, p. 669. "Europae australis."

Barnea (Scobina) costata Dall, Trans. Wagner Free Inst. Sci., 1898, vol. 3, pt. 4, p. 816 .

Pholas costata Gould, Inv. Mass., 1870, p. 36, fig. 363.

Mass.- New Bedford; Woods Hole; Marthas Vineyard.

Conn.-Long Island Sound.

\section{*Barnea truncata (Say).}

Dall, Trans. Wagner Free Inst. Sci., 1898, vol. 3, pt. 4, p. 816.

Pholas truncata Say, Journ. Acad. Nat. Sci. Phila., 1822, ser. 1, vol. 2, p. 321. " southern coast [of United States]." Gould, Inv. Mass., 1870, p. 38, fig. 364.

ME.-Scarboro (Roper).

MAss.-Salem Harbor (Morse); Revere; Vineyard Sound; New Bedford; Duxbury.

R. I.-Bristol.

Conn.- Near New Haven. 


\section{*Zirfaea crispata (Linné).}

Gould, Inv. Mass., 1870, p. 39, fig. 365.

Mya crispata Linné, Syst. Nat., ed. 10, 1758, vol. 1, p. 670. "O. Septentrionali."

Me.-Casco Bay, 10 to 20 fathoms; Frenchman's Bay, 10 fathoms; Eastport.

Mass.- Nahant; Phillips Beach; Woods Hole; Cohasset; Duxbury.

Conn.--Stonington.

\section{*Xylophaga dorsalis (Turton).}

Turton, Conch. Ins. Brit., 1822, p. 253, pl. 2, figs. 4, 5. Forbes and Hanley, Brit. Moll., 1853, vol. 1, p. 90, pl. 2, figs. 3, 4.

Teredo dorsalis Turton, Conch. Dict. Brit. Islands. 1819, p. 185. "Devonshire const."

Me.-Casco Bay, between Bates Island and Mink Rocks; Gulf of Maine, 100 to 110 fathoms.

\section{Martesia cuneiformis (Say).}

Verrill, Rept. Inv. Vineyard Sound, 1873, p. 671. Johnson, Nautilus, 1904, vol. 18, p. 101, fig. 2. Perkins, Proc. Boston Soc. Nat. Hist., 1869, vol. 13, p. 140.

Pholas cuneiformis Say, Journ. Acad. Nat. Sci. Phila., 1822, ser. 1, vol. 2, p. 322. "southern coast [of United States]."

Conn.- New Haven Bay. One specimen of this species in a pile of shells on the beach (Perkins).

\section{TEREDINIDAE.}

Teredo navalis Linné.

Linné, Syst. Nat., ed. 12, 1767, vol. 1, p. 1267. “ "INTRA LIGNUM Navium***ex Indis in Europam propagata." Gould, Inv. Mass., 1870, p. 28, fig. 355. 
MAss.- Vineyard Sound and Buzzards Bay, in cedar buoys; Woods Hole in piles of wharves.

Conn.- New Haven, in piles of wharves.

\section{Teredo norvagica Spengler.}

Tercdo norvagicus Spengler, Skriv. Nat.-Selsk. Kjobenhavn, 1792, vol. 2, pt. 1, p. 102, pl. 2, figs. 4-6. "FriedriKsvaerns HavN I Norge.” Gould, Inv. Mass., 1870, p. 29, fig. 356.

The prevailing indigenous species in the Northern Atlantic seas, but it is rare on the American coast (Gould).

\section{Teredo nana Turton.}

Turton, Conch. Insul. Brit., 1822, p. 16, pl. 2, figs. 6, 7. "'TorBAy, [EnGLAND]."

Teredo megotara Forbes and Hanley, Brit. Conch., 1853, vol. 1, p. 77 , pl. 4, fig. 6 ; pl. 18, figs. 1, 2; vol. 4, p. 246 . Gould, Inv. Mass., 1870, p. 30, fig. 357.

Me.-From driftwood, Ironbound Island, Frenchman's Bay (Blaney).

Mass.-Provincetown; New Bedford; Cohasset.

R. I.- Newport.

\section{Teredo thomsonii Tryon.}

Tryon, Proc. Acad. Nat. Sci. Phila., 1863, p. 280, pl. 2, figs. 3-5. "New Bedford, Mass." Gould, Inv. Mass., 1870, p. 31, fig. 358.

Mass.- New Bedford; Provincetown.

\section{Teredo dilatata Stimpson.}

Stimpson, Proc. Boston Soc. Nat. Hist., 1851, vol. 4, p. 113. “Lrnn, (Mass.)." Gould, Inv. Mass., 1870, p. 32, fig. 359.

Me.- Casco Bay.

Mass.- From pine buoys near Cape Ann; Marblehead; Provincetown. 
Teredo (Lyrodus) chlorotica Gould.

Teredo chlorotica Gould, Inv. Mass., 1870, p. 33, fig. 360. “ "TIMBers of ships that have cruised in the Pacific."

Teredo (Lyrodes) chlorotica Dall, Bull. U. S. Nat. Mus., 1889, no. 37, p. 74 , pl. 68 , fig. 3 .

Mass.- From timbers of ships that have cruised in the Pacific (Gould).

Xylotrya fimbriata (Jeffreys).

Gould, Inv. Mass., 1870, p. 34, fig. 361.

Teredo fimbriata Jeftreys, Ann. Mag. Nat. Hist., 1860, ser. 3, vol. 6, p. 126. "Leith, [England.]"

Iylotrya palmulata Perkins, Proc. Boston Soc. Nat. Hist., 1869, vol. 13, p. 141.

Mass.- Taken from one of the timbers of "Old, Ironsides" (Gould).

Conn.- In an old submerged wreck near New Haven (Verrill).

\section{SCAPHOPODA.}

SOLENCONCHAE.

\section{DENTALIIDAE.}

\section{*Dentalium entalis Linné.}

Linné, Syst. Nat., ed. 10, 1758, vol. 1, p. 785 . “O. Europaeo, INDICO."

Dentalium striolatum Stimpson, Proc. Boston Soc. Nat. Hist., 1851, vol. 4, p. 114 .

Entalis striolata Gould, Inv. Mass., 1870, p. 266, fig. 528.

Me.-Casco Bay; Frenchman's Bay, 25 to 30 fathoms; Eastport, 10 to 20 fathoms.

N. H.- Off Isles of Shoals.

Mass.-Massachusetts Bay. 
Dentalium agile G. O. Sars.

G. O. Sars, Remarkable Forms Anim. Life, 1872, p. 34, pl. 3, figs. 4, 5. [Lofoten, 200 to 300 fathoms.] Pilsbry and Sharp, Manual Conch., 1897, ser. 1, vol. 17, p. 46, pl. 8, fig. 36.

ME.-Casco Bay (Kingsley).

*Dentalium occidentale Stimpson.

Stimpson, Shells of New England, 1851, p. 28. "COAST of Maine, And in Mass. Bay." Pilsbry and Sharp, Manual Conch., 1897, ser. 1, vol. 17, p. 47, pl. 13, figs. 9-11; pl. 9, figs. 41-43.

Dentalium dentale Gould, Inv. Mass., 1841, p. 155, pl. 1, fig. 5 (non Linné).

Me.- Casco Bay; Frenchman's Bay, 10 fathoms; Eastport 10 to 20 fathoms.

Mass.- Massachusetts Bay.

Dentalium occidentale sulcatum Verrill.

Verrill, Trans. Conn. Acad., 1884, vol. 6, p. 217. [ofF CAPE Con.]

Mass.- East of Cape Cod, 75 fathoms.

\section{SIPHONODENTALIIDAE.}

Siphonodentalium lobatum (Sowerby).

Pilsbry and Sharp, Manual Conch., 1897, ser. 1, vol. 17, p. 136, pl. 23, figs. 8-21.

Dentalium lobatum G. B. Sowerby, Jr., Thes. Conch., 1866, vol. 3, p. 100 , pl. 3 , fig. 44 .

Siphonodentalium vitreum Verrill, Trans. Conn. Acad., 1882, vol. 5, p. 557, pl. 42, fig. 19.

Gulf of Maine, 60 to 107 fathoms.

Mass.-Off Marthas Vineyard, 100 to 487 fathoms. 
Siphonodentalium (Pulsellum) lofotense M. Sars.

Siphonodentalium lofotense M. Sars, Forh. Vid.-Selsk. Christiania for 1864, 1865, p. 297, pl. 6, figs. 29-33. “Lofoten.” Pilsbry and Sharp, Manual Conch., 1897, ser. 1, vol. 17, p. 138, pl. 24, figs. $40-44$.

Mass.-Off Marthas Vineyard, 115 to 500 fathoms (Verrill).

Cađulus jeffreysii (Monterosato).

Helomyx jeffreysii Monterosato, Atti Reale Accad. Sci. Palermo, 1875, vol. 5, p. 20. [MEDiterraneAN.]

Cadulus propinquus Verrill, Trans. Conn. Acad., 1882, vol. 5, p. 558, pl. 58, figs. 31, 32 (non Sars).

Cadulus jeffreysi Pilsbry and Sharp, Manual Conch., 1897, ser. 1 , vol. 17 , p. 164 , pl. 24 , fig. 39 ; pl. 32, figs. 44-46.

MAss.-Off Marthas Vineyard, 100 to 115 fathoms (Verrill).

Cadulus pandionis Verrill and Smith.

Verrill and Smith in Verrill, Amer. Journ. Sci., 1880, ser. 3, vol. 20, pp. 392, 399. [off Marthas Vineyard.] Verrill, Trans. Conn. Acad., 1882, vol. 5, p. 558, pl. 58, figs. 30, 30a.

Mass.- South of Marthas Vineyard, 85 to 500 fathoms (Verrill).

\section{Cadulus watsoni Dall.}

Dall, Bull. Mus. Comp. Zoöl., 1881, vol. 9, p. 34. “ofF C. San Antonio *** Yucatan Strait.” Dall, Bull. Mus. Comp. Zoöl., 1889, vol. 18, p. 429, pl. 27, fig. 12a. Verrill, Trans. Conn. Acad., 1884, vol. 6, p. 219.

Mass.-South of Marthas Vineyard, 197 fathoms. 


\section{GASTEROPODA.}

Sub-class STREPTONEURA.

ASPIDOBRANCHIA.

$$
\text { ACMAEIDAE. }
$$

\section{*Acmaea testudinalis (Müller).}

Pilsbry, Manual Conch., 1891, ser. 1, vol. 13, p. 10, pl. 9, figs. 25-29.

Patella testudinalis Müller, Zool. Danicae Prodr., 1776, p. 237. "Daniae et Norvegiae."

Tectura testudinalis Gould, Inv. Mass., 1870, p. 267, fig. 529.

ME.- Common along the entire coast.

Mass.- Marblehead; Cohasset; Provincetown; Marthas Vineyard.

R. I.- Watch Hill.

Conn.- New Haven, rare.

\section{*Acmaea alveus (Conrad).}

Patella alveus Conrad, Journ. Acad. Nat. Sci. Phila., 1831, ser. 1, vol. 6, p. 267, pl. 11, fig. 20. "MAssachusetts."

Tectura alveus Gould, Inv. Mass., 1870, p. 269, fig. 530.

Me.-Casco Bay; North Haven; Frenchman's Bay.

Mass.- Massachusetts Bay.

\section{Acmaea fergusoni Wheat.}

Wheat, Sci. Bull. Brooklyn Inst. Arts and Sci., 1913, vol. 2, p. 17. " NORTH SHORE LONG IsLAND, N. Y."

Conn.- New Haven (Perkins), see Wheat, Nautilus, 1913, vol. $27, \bar{p} .72$. 


\section{LEPTIDAE.}

*Lepeta caeca (Müller).

Gould, Inv. Mass., 1870, p. 270, fig. 531.

Patella caeca Müller, Zool. Danicae Prodr., 1776, p. 237. "Daniae et Norvegiae."

Me.- Casco Bay; Frenchman's Bay, 25 to 30 fathoms; Eastport.

Mass.- From stomach of fish caught off Barnstable.

Lepetella tubicola Verrill and Smith.

Verrill and Smith in Verrill, Amer. Journ. Sci., 1880, ser. 3, vol. 20, p. 396. [off Marthas Vineyard.] Verrill, Trans. Conn. Acad., 1882, vol. 5, p. 534, pl. 58, figs. 29, 29a.

Mass.- Off Marthas Vineyard, in old tubes of Hyalinoecia artifex, 130 to 388 fathoms.

\section{FISSURELLIDAE.}

*Puncturella princeps (Mighels and Adams).

Cemoria princeps Mighels and Adams, Boston Journ. Nat. Hist., 1842, vol. 4, p. 42, pl. 4, fig. 9. “FROM THE STOMACH OF A HADDOCK $* * *$ SEVENTY-FIVE TO ONE HUNDRED MILES OFF THE MOUTH of Kennebec RIVER *** Forty to SEVEnty-Five FAthoms."

Puncturclla noachina Pilsbry, Manual Conch., 1890, ser. 1, vol. 12, p. 229, pl. 27, figs. 69, 70; and authors, non Linné, 1771.

Cemoria noachina Gould, Inv. Mass., 1870, p. 276, fig. 537.

Me.-Casco Bay; Frenchman's Bay, 25 to 30 fathoms; Eastport, 30 fathoms.

Mass.-Georges Bank. 


\section{COCCULINIDAE.}

Cocculina beanii Dall.

Dall, Proc. U. S. Nat. Mus., 1882, vol. 4, p. 403. [South of Marthas Vineyard.] Dall, Bull. Mus. Comp. Zoöl., 1889, vol. 18, p. 347 , pl. 25 , figs. $2,4,8$.

Mass.- South of Marthas Vineyard, 100 to 335 fathoms.

\section{SCISSURELLIDAE.}

Scissurella crispata Fleming.

Fleming, Mem. Wern. Nat. Hist. Soc., 1832, vol. 6, p. 385, pl. 6, fig. 3. "Isle of Noss, Zetrand." Forbes and Hanley, Brit. Moll., 1853, vol. 2, p. 544, pl. 63, fig.6. Dall, Bull. U. S. Nat. Mus., 1889, no. 37, p. 168, pl. 48, fig. 15.

N. H.- Hampton Beach (Morse).

\section{TROCHIDAE.}

*Molleria costulata (Möller).

Margarita? costulata Möller, Krфyer's Naturh. Tidskr., 1842, vol. 4, p. 81. [GREenLAND.]

Adeorbis costulata Gould, Inv. Mass., 1870, p. 278, fig. 538.

ME.-Frenchman's Bay, 20 to 30 fathoms; Eastport, 30 fathoms.

Mass.- Off Cape Ann; off Nantucket, 4 fathoms.

* Margarites helicina (Phipps).

Turbo helicinus Phipps, Voyage toward the North Pole, 1774, p. 198. " on the North side of Spitsbergen."

Margarita arctica Gould, Inv. Mass., 1841, p. 255, fig. 163.

Margarita helicina Gould, Inv. Mass., 1870, p. 281, fig. 542.

ME.- Casco Bay; Frenchman's Bay, low tide to 20 fathoms; Eastport.

MAss.-Duxbury; Plymouth. 


\section{* Margarites olivacea (Brown).}

Turbo olivaceus Brown, Illustr. Conch., 1827, pl. 46, figs. 30, 31. "Greenock, [England]."

Margarita argentata Gould, Inv. Mass., 1870, p. 282, fig. 544.

Me.-Casco Bay; Frenchman's Bay, 25 to 30 fathoms; Eastport.

Mass.-Off Cape Ann and Cohasset.

\section{Margarites acuminata (Sowerby).}

Margarita acuminata Sowerby, Mal. and Conch. Mag., 1838, vol. 1, p. 26. [Arctic Ocean.] Pilsbry, Manual Conch., 1889, ser. 1, vol. 11, p. 287, pl. 39, fig. 55; pl. 66, fig. 6 .

Gulf of Maine; Grand Manan, in 40 fathoms on a soft muddy bottom off the "Swallow's Tail " (Stimpson).

\section{*Margarites groenlandica (Gmelin).}

Trochus groenlandicus Gmelin, Syst. Nat., 1790, vol. 1, pt. 6, p. 3574. "Groenlandiae littora."

Margarita undulata Gould, Inv. Mass., 1870, p. 280, fig. 541.

Me.-Casco Bay; Frenchman's Bay, 6 to 35 fathoms; Eastport. Mass.- Phillips Beach.

\section{* Margarites cinerea (Couthouy).}

Turbo cinereus Couthouy, Boston Journ. Nat. Hist., 1838, vol. 2, p. 99, pl. 3, fig. 9. "vicinity of Cape Ann, Mass."

Margarita cinerea Gould, Inv. Mass., 1870, p. 279, fig. 539.

Me.- Casco Bay; Frenchman's Bay, 6 to 30 fathoms; West Isles, near Eastport, 10 fathoms.

Mass.-From fish caught in Massachusetts Bay.

\section{Margarites multilineata (DeKay).}

Margarita multilineata DeKay, Nat. Hist. N. Y., Moll., 1843, p. 109, pl. 6, fig. 108. "stomachs OF CODFishes on THE COAST." Conn.- Stratford (Linsley). 
Margarites minutissima (Mighels).

Margarita minutissima Mighels, Boston Journ. Nat. Hist., 1843, vol. 4, p. 349, pl. 16, fig. 5. "CAsco BaY." Gould, Inv. Mass., 1870 , p. 280 , fig. 540.

Me.-- Casco Bay, from the stomach of a haddock.

*Solariella obscura (Couthouy).

Pilsbry, Manual Conch., 1889, ser. 1, vol. 11, p. 308, pl. 57, figs. 44,45 .

Turbo obscurus Couthouy, Boston Journ. Nat. Hist., 1838, vol. 2, p. 100, pl. 3, fig. 12. "Waters of Mass. Bay."

Margarita obscura Gould, Inv. Mass., 1870, p. 283, fig. 545.

Me.-Casco Bay; Frenchman's Bay, 6 to 35 fathoms; Eastport, 6 fathoms.

N. H.- Off Isles of Shoals, 20 fathoms.

Mass.-Beverly Harbor; off Egg Rock, near Nahant, 17 fathoms; off Marthas Vineyard, 20 to 25 fathoms.

Conn.- Stonington, from haddock (Linsley).

Solariella obscura bella (Verkrüzen).

Pilsbry, Manual Conch., 1889, ser. 1, vol. 11, p. 310, pl. 64, figs. 57,58 .

Margarita bella Verkrüzen, Jahrb. Deutsch. Mal. Ges., 1875, p. 236. "Hammerfest."

ME.- Eastport, 10 to 40 fathoms.

Georges Bank, 43 to 45 fathoms.

Solariella obscura planula (Verrill).

Machaeroplax obscura var. planula Verrill, Trans. Conn. Acad., 1882, vol. 5, p. 531. " "south of CAPE CoD."

MAsss. - South of Cape Cod, 15 to 30 fathoms (Verrill). 
Solariella obscura carinata (Verrill).

Machaeroplax obscura var. carinata Verrill, Trans. Conn. Acad., 1882, vol. 5, p. 532. "off Martha's Vineyard."

Mass.-Off Marthas Vineyard, 146 to 335 fathoms.

Solariella lamellosa (Verrill and Smith).

Pilsbry, Manual Conch., 1889, ser. 1, vol. 11, p. 315, pl. 57, fig. 14. Margarita lamellosa Verrill and Smith in Verrill, Amer. Journ. Sci., 1850, ser. 3, vol. 20, pp. 392, 397. [off Marthas Vineyard.]

Mass.-South of Marthas Vineyard, 100 to 192 fathoms.

Solariella ottoi (Philippi).

Pilsbry, Manual Conch., 1889, ser. 1, vol. 11, p. 320, pl. 57, fig. 17.

Trochus ottoi Philippi, Enum. Moll. Siciliae, 1844, vol. 2, p. 227, pl. 28, fig. 9. " prope Messinam."

Margarita regalis Verrill and Smith in Verrill, Amer. Journ. Sci., 1880, ser. 3, vol. 20, pp. 392, 397.

MAss.- South of Marthas Vineyard, 115 to 500 fathoms.

${ }^{*}$ Calliostoma occidentale (Mighels and Adams).

Trochus occidentalis Mighels and Adams, Boston Journ. Nat. Hist., 1842, vol. 4, p. 47, pl. 4, fig. 16. "CASco BaY."

Calliostoma occidentalis Pilsbry, Manual Conch., 1889, ser. 1, vol. 11, p. 393, pl. 37, figs. 2, 3.

Me.-Casco Bay; Penobscot Bay; Frenchman's Bay; Eastport.

Calliostoma bairdii Verrill and Smith.

Verrill and Smith in Verrill, Amer. Journ. Sci., 1880, ser. 3, vol. 20, pp. 391, 396. [south of Marthas Vineyard.] Verrill, Trans. Conn. Acad., 1882, vol. 5, p. 530, pl. 57, fig. 26.

Mass.- South of Marthas Vineyard, 65 to 192 fathoms. 
CTENOBRANCHIATA.

$$
\text { EULIMIDAE. }
$$

Melanella (Eulima) oleacea (Kurtz and Stimpson).

Eulima oleacea Kurtz and Stimpson, Proc. Boston Soc. Nat. Hist., 1851, vol. 4, p. 115. "Buzzard's Bay." Gould, Inv. Mass., 1870, p. 332, fig. 603.

Mass.- In Vineyard Sound it is not uncommon on Thyone briareus in 4 to 10 fathoms (Verrill); Buzzards Bay, 8 fathoms.

Melanella (Eulima) conoidea (Kurtz and Stimpson).

Eulima conoidea Kurtz and Stimpson, Proc. Boston Soc. Nat. Hist., 1851, vol. 4, p. 115. "North and South Carolina."

Mass.- Vineyard Sound and Buzzards Bay (Sumner); Woods Hole (Winkley).

Melanella (Eulima) stenostoma (Jeffreys).

Eulima stenostoma Jeffreys, Ann. Mag. Nat. Hist., 1858, ser. 3, vol. 2, p. 128, pl. 5, fig. 7. "Zetland" [= Shetland].

Mass.- Woods Hole (Winkley).

* Melanella (Eulima) sarsi (Bush).

Eulima intermedia Verrill, Amer. Journ. Sci., 1880, ser. 3, vol. 20, p. 392 (non Cantraine, 1835). [south of Marthas Vineyard.] Verrill, Trans. Conn. Acad., 1882, vol. 5, p. 535, pl. 58, fig. 20.

Eulima sarsi Bush, Amer. Journ. Sci., 1909, ser. 4, vol. 27, p. 479.

Mass.- South of Marthas Vineyard, 85 to 155 fathoms.

Melanella (Eulima) perversa (Bush).

Eulima distorta Verrill, Amer. Journ. Sci., 1880, ser. 3, vol. 20, p. 392 (non Deshayes, non Sars). [south of Marthas Vineyard.] Eulima perversa Bush, Amer. Journ. Sci., 1909, ser. 4, vol. 27, p. 479 .

Mass.- - South of Marthas Vineyard, 115 fathoms. 
Stylifer stimpsonii Verrill.

Verrill, Amer. Journ. Sci., 1872, ser. 3, vol. 3, pp. 210, 283. "off*** New Jersey, on a Bank in 32 fathoms." Verrill, Trans. Conn. Acad., 1882, vol. 5, p. 535, fig. 2.

Lives among the spines of Strongylocentrotus dröbachiensis.

Mass.- Off Gay Head (Sumner); Georges Bank, 60 fathoms. R. I.-Off Block Island, 13 to 27 fathoms.

\section{PYRAMIDELLIDAE.}

Pyramidella (Syrnola) smithii (Verrill).

Turbonilla smithii Verrill, Proc. U. S. Nat. Mus., 1880, vol. 3, p. 380. [south of Marthas Vineyard.]

Eulimella smithii Verrill, Trans. Conn. Acad., 1882, vol. 5, p. 538, pl. 58, fig. 18.

Pyramidella (Syrnola) smithi Bartsch, Proc. Boston Soc. Nat. Hist., 1909, vol. 34, p. 71, pl. 11, figs. 11, 15.

South of Marthas Vineyard in 85 to 146 fathoms.

Pyramidella (Syrnola?) producta (C. B. Adams).

Bartsch, Proc. Boston Soc. Nat. Hist., 1909, vol. 34, p. 72, pl. 11, fig. 3.

Jaminia producta C. B. Adams, Boston Journ. Nat. Hist., 1840, vol. 3, p. 322, pl. 3, fig. 8. "Fairhaven, [Mass.]."

Odostomia producta Gould, Inv. Mass., 1870, p. 325, fig. 593.

Me.

Mass.- Massachusetts Bay; Woods Hole; Fairhaven; Chatham; Duxbury.

R. I.

Conn.- New Haven; Stonington; Stratford; Branford River.

Pyramidella (Syrnola?) fusca (C. B. Adams).

Bartsch, Proc. Boston Soc. Nat. Hist., 1909, vol. 34, p. 73, pl. 11, fig. 4. 
Pyramis fusca C. B. Adams, Boston Journ. Nat. Hist., 1839, vol. 2, p. 282, pl. 4, fig. 9. "NEw Bedford, [Mass.]."

Odostomia fusca Gould, Inv. Mass., 1870, p. 325, fig. 594.

ME.-- Damariscotta.

Mass-Dassachusetts Bay; Fairhaven; New Bedford; Woods Hole; Naushon Id.

R. I.- Tiverton; Watch Hill.

Conn.--Stonington; New Haven; Stratford; Branford River.

\section{*Pyramidella (Syrnola?) winkleyi Bartsch.}

Bartsch, Proc. Boston Soc. Nat. Hist., 1909, vol. 34, p. 74, pl. 11, fig. 1. "Branford River, Conn."

Mass.-Chatham.

Conn.- Branford River (H. W. Winkley).

Turbonilla (Ptycheulimella?) polita (Terrill).

Bartsch, Proc. Bostoni Soc. Nat. Hist., 1909, vol. 34, p. 75.

Aclis polita Verrill, Amer. Journ. Sci., 1872, ser. 3, vol. 3, pp. 210, 282, pl. 6, fig. 5. "Eastport Harbor, [Me.]."

Eulimella polita Verrill, Trans. Conn. Acad., 1882, vol. 5, p. 538, fig. 4 .

Eulimella ventricosa Verrill, Proc. U. S. Nat. Mus., 1880, vol. 3, p. 380 (in part; non Forbes).

ME.- Eastport, 20 fathoms; Isle au Haute.

Turbonilla stricta Verrill.

Verrill, Rept. Inv. Vineyard Sound, 1873, p. 659. "Long Island Sound, off New Haven, Connecticut." Bartsch, Proc. Boston Soc. Nat. Hist., 1909, vol. 34, p. 76, pl. 11, figs. 6, 7.

Mass.- Woods Hole; Naushon Id.; Buzzards Bay.

Conn.- Off New Haven, 3 to 8 fathoms.

*Turbonilla nivea (Stimpson).

Chemnitzia nivea Stimpson, Proc. Boston Soc. Nat. Hist., 1851, vol. 4, p. 114. "off Grand Manan." 
Turbonilla (Turbonilla) nivea Bartsch, Proc. Boston Soc. Nat. Hist., 1909, vol. 34, p. 77, pl. 11, figs. 9, 10.

Me.-Frenchman's Bay; off Grand Manan, 40 fathoms.

Mass.- Woods Hole; off Marthas Vineyard, 100 fathoms.

Conn.- - New Haven (Perkins).

\section{Turbonilla (Chemnitzia) aequalis (Say).}

Bartsch, Proc. Boston Soc. Nat. Hist., 1909, vol. 34, p. 78.

Turritella aequalis Say, Journ. Acad. Nat. Sci. Phila., 1827, ser. 1, vol. 5, p. 208. [United States.]

Mass.-Vineyard Sound, 6 to 8 fathoms.

Turbonilla (Pyrgiscus) verrilli Bartsch.

Bartsch, Proc. Boston Soc. Nat. Hist., 1909, vol. 34, p. 82, pl. 12, figs. 21, 26. "Martha's Vineyard, Mass."

Mass.- Marthas Vineyard; Woods Hole.

*Turbonilla (Pyrgiscus) vineae Bartsch.

Bartsch, Proc. Boston Soc. Nat. Hist., 1909, vol. 34, p. 83, pl. 12, figs. 22, 36. "Wood's Holl, Mass., *** Branford, Conn."

Mass.-Woods Hole.

Conn.- Branford (H. W. Winkley).

Turbonilla (Pyrgiscus) elegantula Verrill.

Bartsch, Proc. Boston Soc. Nat. Hist., 1909, vol. 34, p. 84, pl. 12, figs. 30,31 .

Turbonilla elegans Verrill, Amer. Journ. Sci., 1872, ser. 3, vol. 3, p. 282, pl. 6, fig. 4 (non Wood, 1842). "Vineyard Sound."

Turbonilla elegantula Verrill, Trans. Conn. Acad., 1882, vol. 5, p. 538 .

Mass.-- Vineyard Sound, 2 to 10 fathoms; Woods Hole ("Eel Pond"). 
R. I.- Narragansett Bay.

Conn.-Off New Haven, 2 to 6 fathoms; off Old Tower, 9 fathoms.

Turbonilla (Pyrgiscus) elegantula branfordensis Bartsch.

Bartsch, Proc. Boston Soc. Nat. Hist., 1909, vol. 34, p. 85, pl. 12, fig. 27. "Branford, Conn."

Mass.-Swampscott; Woods Hole.

Conn.-Branford.

*Turbonilla (Pyrgiscus) areolata Verrill.

Bartsch, Proc. Boston Soc. Nat. Hist., 1909, vol. 34, p. S6, pl. 12, figs. 19, 24.

Turbonilla areolata Verrill, Rept. Inv. Vineyard Sound, 1873, p. 658. "NEAR New Haven [Conn.]."

Mass.- Vineyard Sound, 2 to 8 fathoms; Woods Hole.

R. I.-- Block Island.

Cons.- Long Island Sound, near New Haven; Branford.

*Turbonilla (Pyrgiscus) interrupta (Totten).

Bartsch, Proc. Boston Soc. Nat. Hist., 1909, vol. 34, p. 87, pl. 12, figs. 18, 23.

Turritella interrupta Totten, Amer. Journ. Sci., 1835, ser. 1, vol. 28, p. 352, fig. 7. "Newport harbor, (R. I.)."

Turbonilla interrupta Gould, Inv. Mass., 1870, p. 331 (not fig. $601)$.

Turbonilla pseudointerrupta Bush, Amer. Journ. Sci., 1909, ser. 4 , vol. 27 , p. 481 , fig. 9 .

ME. - ?Portland Harbor, dead shell (Morse).

Mass.- Woods Hole; Vineyard Sound and Buzzards Bay, 3 to 10 fathoms.

R. I.- Narragansett Bay; Newport.

Conn.- Off Thimble Island and New Haven, 3 to 5 fathoms; Stonington. 
Turbonilla (Pyrgiscus) mighelsi Bartsch.

Bartsch, Proc. Boston Soc. Nat. Hist., 1909, vol. 34, p. 88, pl. 14, figs. 52,54 .

Turbonilla costulata Verrill, Rept. Inv. Vineyard Sound, 1873, p. 658 (non Risso, 1826). "Near New Haven, Conn."

Mass.- Vineyard Sound, 1 to 5 fathoms; Woods Hole.

Conn.- Long Island Sound, near New Haven.

Turbonilla (Pyrgiscus) buteonis Bartsch.

Bartsch, Proc. Boston Soc. Nat. Hist., 1909, vol. 34, p. 89, pl. 12, figs. 17, 28, 34. "Wood's Holl, Mass."

Mass.-Massachusetts Bay; Woods Hole; Marthas Vineyard.

*Turbonilla (Pyrgiscus) winkleyi Bartsch.

Bartsch, Proc. Boston Soc. Nat. Hist., 1909, vol. 34, p. 90, pl. 12. figs. 35, 37. "Wood's Holl, Mass."

Mass.-Woods Hole; Duxbury; Salem.

Conn.- Branford; off Penfield Reef, Long Island Sound, 5 to 10 fathoms.

Turbonilla (Pyrgiscus) winkleyi senilis Bartsch.

Bartsch, Proc. Boston Soc. Nat. Hist., 1909, vol. 34, p. 92, pl. 12, fig. 41. "Branford, Conn."

Mass.- Woods Hole.

Conn.- Branford.

Turbonilla (Pyrgiscus) sumneri Bartsch.

Bartsch, Proc. Boston Soc. Nat. Hist., 1909, vol. 34, p. 92, pl. 14, figs. 53, 55. "Wood's Holl, Mass."

Mass.-Woods Hole. 
Turbonilla (Pyrgiscus) rathbuni Verrill and Smith.

Bartsch, Proc. Boston Soc. Nat. Hist., 1909, vol. 34, p. 94, pl. 12, figs. 33,39 .

Turbonilla rathbuni Verrill and Smith in Verrill, Amer. Journ. Sci., 1880, ser. 3, vol. 20, p. 398. [off Marthas Vineyard, 192 FATHOMS.]

Mass.- Woods Hole; south of Marthas Vineyard, 64 to 321 fathoms.

R. I.- Newport.

Turbonilla (Pyrgiscus) cascoensis Bartsch.

Bartsch, Proc. Boston Soc. Nat. Hist., 1909, vol. 34, p. 95, pl. 12, figs. 29, 40. "CASCO BAY, ME."

ME.- Casco Bay.

*Odostomia (Chrysallida) seminuda (C. B. Adams).

Bartsch, Proc. Boston Soc. Nat. Hist., 1909, vol. 34, p. 97, pl. 13, figs. 45,48 .

Jaminia seminuda C. B. Adams, Boston Journ. Nat. Hist., 1839, vol. 2, p. 280, pl. 4, fig. 13 (not fig. 8). [Dartmouth Harbor, MAss.]

Mass.-Massachusetts Bay; Vineyard Sound and Buzzards Bay, 2 to 10 fathoms; Woods Hole.

R. I.- Off Newport, 12 fathoms; Sakonnet River, 3 to 11 fathoms.

Conn.- Near New Haven; Branford; Stratford.

Odostomia (Chrysallida) bushiana Bartsch.

Bartsch, Proc. Boston Soc. Nat. Hist., 1909, vol. 34, p. 99, pl. 13, fig. 44. "Narragansett Bay."

Mass. - Vineyard Sound.

R. I.- Narragansett Bay, station 816, 10 fathoms. 
Odostomia (Evalea) bartschi (Winkley).

Winkley, Nautilus, 1912, vol. 26, p. 54.

Pyramidella (Sulcorinella) bartschi Winkley, Nautilus, 1909, vol. 23, p. 39, fig. "Woods Holl, Mass."

Mass.-Woods Hole; Chatham; Duxbury; North Falmouth.

*Odostomia (Evalina) winkleyi Bartsch.

Bartsch, Proc. Boston Soc. Nat. Hist., 1909, vol. 34, p. 100, pl. 13, fig. 47. "Branford, Conn."

Me.-Quahog Bay (Winkley).

Mass.-Rowley; Danvers; Duxbury.

Conn.- Branford.

*Odostomia (Evalina) katherinae Winkley.

Winkley, Nautilus, 1909, vol. 23, p. 88. “Danvers River, [Mass.]."

Mass.-Danvers.

Odostomia (Iolaea) hendersoni Bartsch.

Bartsch, Proc. Boston Soc. Nat. Hist., 1909, vol. 34, p. 101, pl. 13 , fig. 43. "Wood's Holl, Mass."

Mass.-Woods Hole.

Odostomia (Menestho) impressa (Say).

Bartsch, Proc. Boston Soc. Nat. Hist., 1909, vol. 34, p. 103, pl. 13 , fig. 51.

Turritella impressa Say, Journ. Acad. Nat. Sci. Phila., 1822, ser. 1, vol. 2, p. 244. " Maryland."

Mass.- Massachusetts Bay (Stimpson); New Bedford (Stimpson).

ConN.- - Near New Haven. 
Odostomia (Menestho) sulcosa (Mighels).

Phasianella sulcosa Mighels, Boston Journ. Nat. Hist., 1843, vol. 4, p. 348, pl. 16, fig. 4. "CAsco BAY."

Rissoella sulcosa Gould, Inv. Mass., 1870, p. 297, fig. 565 (fig. 570 ?).

Odostomia (Menestho) sulcata Verrill, Proc. U. S. Nat. Mus., 1880, vol. 3, p. 380 .

Menestho sulcata Verrill, Trans. Conn. Acad., 1882, vol. 5, p. 539, pl. 58, fig. 17 (non A. Adams, 1860).

Odostomia (Mencstho) morseana Bartsch, Proc. Boston Soc. Nat. Hist., 1909, vol. 34, p. 104.

Mass.- Off Marthas Vineyard, 115 and 365 fathoms.

*Odostomia (Menestho) trifida (Totten).

Bartsch, Proc. Boston Soc. Nat. Hist., 1909, vol. 34, p. 104, pl. 13, fig. 46.

Acteon trifidus Totten, Amer. Journ. Sci., 1834, ser. 1, vol. 26, p. 368, figs. 4a, 4b. "Rhode Island."

ME.-Newcastle; Sheepscott River.

Mass.-Lynn; Massachusetts Bay; Woods Hole; New Bedford; Weepecket Id.

R. I.-Newport; Kettle Point, Providence River; Oakland Beach.

Conn.- New Haven; Long Beach; Branford.

*Odostomia (Menestho) bisuturalis (Say).

Bartsch, Proc. Boston Soc. Nat. Hist., 1909, vol. 34, p. 106, pl. 14, fig. 59 .

Turritella bisuturalis Say, Journ. Acad. Nat. Sci. Phila., 1822, ser. 1, vol. 2, p. 244. "Boston Harbour, [MAss.]."

ME.- Sheepscott River; Newcastle; Wiscasset; Biddeford Pool.

Mass.-Chelsea; Cohasset; Provincetown; Woods Hole; Naushon Id; Vineyard Sound. 
R. I.- Oakland Beach.

Conn.- Branford; New Haven (Savin Rock).

Odostomia (Menestho) bisuturalis ovilensis Bartsch.

Bartsch, Proc. Boston Soc. Nat. Hist., 1909, vol. 34, p. 107, pl. 14, fig. 57. "Sheepscott River, Maine."

ME.-Sheepscott River; Wiscasset.

Mass - - Duxbury.

Odostomia modesta (Stimpson).

Chemnitzia modesta Stimpson, Proc. Boston Soc. Nat. Hist., 1851, vol. 4, p. 16. "ST. Georges Bank."

Odostomia (Odostomia) modesta Bartsch, Proc. Boston Soc. Nat. Hist., 1909, vol. 34, p. 108, pl. 13, fig. 50.

ME.-Frenchman's Bay, 6 to 8 fathoms (Blaney).

Mass.- Woods Hole; south of Marthas Vineyard, 115 fathoms; Duxbury.

Odostomia gibbosa Bush.

Bush, Amer. Journ. Sci., 1909, ser. 4, vol. 27, p. 482.

Odostomia (Odostomia) modesta Bartsch, Proc. Boston Soc. Nat. Hist., 1909, vol. 34, p. 108, pl. 13, fig. 50 (non Stimpson). "Wood's Holl, Mass."

Me.-Quahog Bay (Winkley).

Mass.-Woods Hole; Duxbury; Provincetown.

Odostomia dealbata (Stimpson).

Gould, Inv. Mass., 1870, p. 327, fig. 595.

Chemnitzia dealbata Stimpson, Proc. Boston Soc. Nat. Hist., 1851, vol. 4, p. 114. "Boston Harbor [, Mass.]."

Odostomia (Odostomia) dealbata Bartseh, Proc. Boston Soc. Nat. Hist., 1909, vol. 34, p. 108.

Mass.- Boston Harbor, 3 fathoms; Duxbury.

Conn.- Near New Haven (Perkins). 
*Cremula eburnea (Stimpson).

Iredale, Proc. Mal. Soc. London, 1915, vol. 11, p. 336.

Rissoa eburnea Stimpson, Proc. Boston Soc. Nat. Hist., 1851, vol. 4, p. 14. "off CAPE Ann, [MAss.]." Stimpson, Shells of New England, 1851, p. 34, pl. 1, fig. 1.

Rissoella? eburnea Gould, Inv. Mass., 1870, p. 297 (not fig. 564). Odostomia (Liostomia) eburnea Bartsch, Proc. Boston Soc. Nat. Hist., 1909, vol. 34, p. 109.

Me.-Casco Bay; Frenchman's Bay, 6 to 8 fathoms (Blaney); off Grand Manan, 25 fathoms (Stimpson).

Mass. - Off Cape Ann, 30 fathoms (Stimpson); Duxbury.

*Couthouyella striatula (Couthouy).

Bartsch, Proc. Boston Soc. Nat. Hist., 1909, vol. 34, p. 110, pl. 11, figs. 13, 16; pl. 14, figs. 56, 58.

Pyramis striatulus Couthouy, Boston Journ. Nat. Hist., 1838, vol. 2, p. 101, pl. 1, fig. 6. "vicinity of CaPe Ann [, Mass.]."

Menestho albula Gould, Inv. Mass., 1870, p. 333, fig. 604 (non Fabricius, 1780).

MIe.-Casco Bay; Eastport; Frenchman's Bay, 20 to 30 fathoms; Cashe's Ledge, 46 fathoms.

N. H.- Isles of Shoals, 20 fathoms.

Mass.- Annisquam Harbor; Gloucester Harbor, 10 fathoms; off Baker's Id., 20 fathoms; off Cape Cod, 15 to 46 fathoms; Buzzards Bay.

Conn.- Bridgeport, from the stomachs of ducks (Linsley).

\section{JANTHINIDAE.}

Janthina janthina (Linné).

Hedley, Bull. New Zealand Colonial Mus., 1906, no. 1, p. 49.

Helix janthina Linné, Syst. Nat., ed. 12, 1767, vol. 1, p. 1246.

"Europa, Asta, Africa." 
Ianthina fragilis Tryon, Manual Conch., 1887, ser. 1, vol. 9, p. 36, pl. 9, figs. 94,95 ; pl. 10, figs. $6-10$.

MAss.-After a severe gale, in the autumn of 1839, great numbers of them were collected on the shores of Nantucket (Gould).

\section{EPITONIIDAE.}

*Epitonium novangliae (Couthouy).

Scalaria novangli (sic in text, but novangliae on plate) Couthouy, Boston Journ. Nat. Hist., 1838, vol. 2, p. 96, pl. 3, fig. 5. " Mass[AChusetts] BaY." Gould, Inv. Mass., 1870, p. 311.

Mass.- Cape Ann, from the stomach of a fish.

\section{*Epitonium lineatum (Say).}

Scalaria lineata Say, Journ. Acad. Nat. Sci. Phila., 1822, ser. 1, vol. 2, p. 242. " southern coast [of United States]." Gould, Inv. Mass., 1870, p. 312 (not fig. 580).

MAss.-Vineyard Sound; Buzzards Bay; New Bedford.

R. I.- Bristol.

Conn.-- New Haven.

\section{Epitonium multistriatum (Say).}

Scalaria multistriata Say, Journ. Acad. Nat. Sci. Phila., 1826, ser. 1, vol. 5, p. 208. "Southern Coast [of United States]." Gould, Inv. Mass., 1870, p. 313, fig. 581.

Mass.-Vineyard Sound; Buzzards Bay; Dartmouth Harbor. R. I.- Off Watch Hill; Block Island.

Conn.- New Haven.

\section{Epitonium humphreysii (Kiener).}

Scalaria humphreysii Kiener, Iconogr. Coq. Viv. (Gen. Scalaire), 1845, p. 15, pl. 5, fig. 16. "Atlantique au sud de la CaroLine." Gould, Inv. Mass., 1870, fig. 580, as S. lineata, on p. 312. 
Scala sayana Dall, Bull. Mus. Comp. Zoöl., 1889, vol. 18, p. 309; Búll. U. S. Nat. Mus., 1889, no. 37, p. 122, pl. 50, fig. 10.

Mass.

ConN.- Stonington.

\section{Epitonium angulatum (Say).}

Scalaria clathrus var. angulata Say, Amer. Conch., 1831, no. 3, "Scalaria," pl. 27, two upper figs. [North America.] Verrill, Rept. Inv. Vineyard Sound, 1873, p. 660.

Scala angulata Dall, Bull. U. S. Nat. Mus., 1889, no. 37, p. 122.

Conn.--Stonington (Linsley).

Epitonium dallianum (Verrill and Smith).

Scalaria dalliana Verrill and Smith in Verrill, Amer. Journ. Sci., 1850, ser. 3, vol. 20, pp. 391, 395. [south of Marthas Vineyard.] Verrill, Trans. Conn. Acad., 1882, vol. 5, p. 527, pl. 57, fig. 33.

Mass.- South of Marthas Vineyard, 85 to 192 fathoms.

\section{Epitonium pourtalesii (Verrill and Smith).}

Scalaria pourtalesii Verrill and Smith in Verrill, Amer. Journ. Sci., 1S80, ser. 3, vol. 20, pp. 391, 395. [sovtil of Marthas Vineyard.] Verrill, Trans. Conn. Acad., 1882, vol. 5, p. 527, pl. 57, fig. 32.

MAss.- South of Marthas Vineyard, 85 to 146 fathoms.

\section{Epitonium leeanum (Verrill).}

Scalaria (Cirsotrema) leeana Verrill, Trans. Conn. Acad., 1882, vol. 5, p. 526, pl. 57, fig. 34. “off Martha's Vineyard."

Mass.-- South of Marthas Vineyard, 146 fathoms.

\section{*E'pitonium (Arctoscala) greenlandicum (Perry).}

Scalaria greenlandica Perry, Conch. or Nat. Hist. Shells, 1811, pl. 28, fig. 8 and explanation. "Greenland." 
Scalaria subulata Couthouy, Boston Journ. Nat. Hist., 1838, vol. 2, p. 93, pl. 3, fig. 4 .

Borcoscala groenlandica Sumner, Osburn and Cole, Bull. U. S. Bureau Fisheries, 1913, vol.,31, pt. 2, p. 714.

Me.- Casco Bay; Frenchman's Bay, 10 to 35 fathoms; Eastport, 10 fathoms.

N. H.- Isles of Shoals, 20 fathoms.

Mass.-Phillips Beach; off Nahant, 17 fathoms; Nantucket Shoals, 20 fathoms.

R. I.- Block Island Sound, 17 to 24 fathoms.

Epitonium (Opalia) andrewsii (Verrill).

Scalaria (Opalia) andrewsii Verrill, Trans. Conn. Acad., 1882, vol. 5, p. 526, pl. 57, fig. 35 . " off Newport, R. I."

R. I.- Off Newport, 100 fathoms (Verrill).

Epitonium (Acirsa) costulatum (Mighels and Adams).

Turritella costulata Mighels and Adams, Boston Journ. Nat. Hist., 1842, vol. 4, p. 50, pl. 4, fig. 20. "CAsco BAY."

Acirsa borealis Verrill, Amer. Journ. Sci., 1872, ser. 3, vol. 3, p. 281.

Scala (Acirsa) costulata Dall, Bull. Mus. Comp. Zoöl., 1889, vol. 18 , p. 307 .

ME.-Casco Bay, from the stomach of a haddock; Eastport, 10 to 40 fathoms.

Epitonium (Acirsa) gracilis (Verrill).

Acirsa gracilis Verrill, Proc. U. S. Nat. Mus., 1880, vol. 3, p. 377. [south of Marthas Vineyard.] Verrill, Trans. Conn. Acad., 1882, vol. 5, p. 528 , pl. 57 , fig. 31.

MASS.- South of Marthas Vineyard, 100 to 365 fathoms. 
Aclis striata Verrill.

Verrill, Proc. U. S. Nat. Mus., 1880, vol. 3, p. 377. "ofF Newport, R. I.” Verrill, Trans. Conn. Acad., 1882, vol. 5, p. 528, pl. 58, fig. 13 .

ME.- Near Eastport.

Mass.- South of Newport, 100 fathoms.

Aclis tenuis Verrill.

Verrill, Trans. Conn. Acad., 1882, vol. 5, p. 528, pl. 58, fig. 19. "ofF Martha's Vineyard."

Eulimella ventricosa Verrill, Proc. U. S. Nat. Mus., 1880, vol. 3, p. 380 (in part; non Forbes).

Mass. - South of Marthas Vineyard, 100 fathoms.

\section{NATICIDAE.}

*Natica (Cryptonatica) clausa Broderip and Sowerby.

Yatica clausa Broderip and Sowerby, Zool. Journ., 1829, vol. 4, p. 372. No locality. Gould, Inv. Mass., 1870, p. 342, fig. 612.

ME.-Casco Bay; Frenchman's Bay, 25 to 30 fathoms; Eastport.

Mass.- Massachusetts Bay; Duxbury.

*Natica (Cryptonatica) pusilla Say.

Natica pusilla Say, Journ. Acad. Nat. Sci. Phila., 1822, ser. 1, vol. 2, p. 257. " southern COAst [of United States]." Gould, Inv. Mass., 1870, p. 344, fig. 613.

Me.- Casco Bay.

Mass.- Vineyard Sound and Buzzards Bay, 2 to 10 fathoms.

*Polinices (Neverita) duplicata (Say).

Natica duplicata Say, Journ. Acad. Nat. Sci. Phila., 1822, ser. 1, vol. 2, p. 247. "COASt OF the United States."

Neverita duplicata Gould, Inv. Mass., 1870, p. 345, fig. 615. 
Mass.- Revere; Provincetown; Vineyard Sound; Nantucket.

R. I.-Nayatt; Buttonwoods.

Conn.-Stratford.

*Polinices (Euspira) heros (Say).

Natica heros Say, Journ. Acad. Nat. Sci. Phila., 1822, ser. 1, vol. 2, p. 248. "NEW Jersey."

Lunatia heros Gould, Inv. Mass., 1870, p. 338, figs. 608, 609.

Me. to Conn.- Common from low water to 40 fathoms.

*Polinices (Euspira) triseriata (Say).

Natica triseriata Say, Journ. Acad. Nat. Sci. Phila., 1826, ser. 1, vol. 5, p. 209. "Boston harbour."

Lunatia triseriata Gould, Inv. Mass., 1870, p. 340, fig. 610.

Me. to Conn.- Common from low water to 30 fathoms.

*Polinices (Euspira) immaculata (Totten).

Natica immaculata Totten, Amer. Journ. Sci., 1835, ser. 1, vol. 28, p. 351, fig. 6. "NEwPoRT HARBoR, (R. I.)."

Mamma? immaculata Gould, Inv. Mass., 1870, p. 344, fig. 614.

Me.- Casco Bay; Frenchman's Bay, 25 to 50 fathoms; Eastport, 20 fathoms.

Mass.-- Off Marthas Vineyard, 20 fathoms; Duxbury; Georges Bank.

R. I.-East of Block Island, 29 fathoms; off Watch Hill.

Conn.- - Stonington, from cod.

*Polinices (Euspira) groenlandica (Möller).

Natica groenlandica Möller, Kŕryyer's Naturh. Tidskr., 1842, vol.4, p. 80. [GreENLAND.]

Lunatia groenlandica Gould, Inv. Mass., 1870, p. 341, fig. 611.

Me.-Frenchman's Bay, 35 fathoms.

Mass.-Massachusetts Bay, from fishes. 
Polinices (Euspira) nana (Möller).

Natica nana Möller, Krøyer's Naturh. Tidskr., 1842, vol. 4, p. 80. [Greenland.]

Lunatia nana Verrill, Trans. Conn. Acad., 1882, vol. 5, p. 516, pl. 42 , fig. 9 .

Me.- Casco Bay; Eastport.

Mass.-Off Cape Ann, 115 fathoms; Stellwagen Bank, 26 to 32 fathoms.

R. I.- South of Block Island, 22 to 29 fathoms.

Polinices (Euspira) levicula (Verrill).

Lunatia levicula Verrill, Proc. U. S. Nat. Mus., 1880, vol. 3, p. 371. "Eastport, Me."

ME.-Casco Bay; Eastport.

Mass.- Off Marthas Vineyard, 26 fathoms.

R. I.- Off Block Island, 26 to 100 fathoms.

*Amauropsis islandica (Gmelin).

Nerita islandica Gmelin, Syst. Nat., 1790, vol. 1, pt. 6, p. 3675. "Oceano septentrionali."

Amauropsis helicoides Gould, Inv. Mass., 1870, p. 348, fig. 617.

ME.-Off Otter Creek, Mt. Desert, 27 fathoms (Henderson).

Mass.-One fine specimen from a fish caught in Massachusetts Bay (Gould); Georges Bank.

*Acrybia smithii (Brown).

Bulbus smithii Brown, Mem. Wern. Nat. Hist. Soc., 1839, vol. 8, p. 104, pl. 1, fig. 18. [Marine pleistocene, Ardincaple, ScotLAND.]

Natica flava Gould, Amer. Journ. Sci., 1840, ser. 1, vol. 38, p. 196.

Bulbus flavus Gould, Inv. Mass., 1870, p. 347, fig. 616.

Ampullina smithii G. O. Sars, Moll. Reg. Arct. Norv., 1878, p. 155 , pl. 12, fig. 2; pl. 21, fig. 18 . 
ME.- Eastport; "Grand Manan, 50 fathoms, off the Swallow Tail " (Stimpson).

Mass.-Georges Bank.

Conn.- Stonington, from haddock (Linsley).

\section{I.AMELLARIIDAE.}

Lamellaria pellucida Verrill.

Verrill, Amer. Journ. Sci., 1880, ser. 3, vol. 20, pp. 391, 395. [south of Marthas Vineyard.] Verrill, Trans. Conn. Acad., 1882, vol. 5, p. 518, pl. 58, figs. 4, 5, 5a.

Mass.- South of Marthas Vineyard, 86 to 155 fathoms.

*Velutina laevigata (Linné).

Helix laevigata Linné, Syst. Nat., ed. 12, 1767, vol. 1, p. 1250. No locality.

Velutina haliotoidea Gould, Inv. Mass., 1870, p. 334, fig. 605.

Me.-Frenchman's Bay, 10 to 50 fathoms; Eastport, 20 fathoms.

Mass.--Marblehead; Magnolia; Duxbury.

*Velutina undata Brown.

Brown, Mem. Wern. Nat. Hist. Soc., 1839, vol. 8, p. 102, pl. 1, fig. 15. [Pleistocene, Dalmuir, Scotland.]

Velutina zonata Gould, Inv. Mass., 1870, p. 335, fig. 606.

ME.-Frenchman's Bay, 25 to 30 fathoms; Eastport, 20 fathoms; Casco Bay.

Mass.-From fish caught off Cape Ann; Chelsea Beach (Gould).

*Marsenina glabra (Couthouy).

Verrill, Trans. Conn. Acad., 1882, vol. 5, p. 517, pl. 42, figs. 1, $1 \mathrm{a}, 4$, 
Oxinoe? glabra Couthouy, Boston Journ. Nat. Hist., 1832, vol. 2, p. 90, pl. 3, fig. 16. "Mass[AChusetTs] BAY."

Lamellaria perspicua Gould, Inv. Mass., 1870, p. 337, fig. 607.

ME.- Eastport.

Mass.-- Off Cape Cod, 34 fathoms; Georges Bank.

Marsenina prodita (Lovén).

Verrill, Trans. Conn. Acad., 1882, vol. 5, p. 51S, pl. 42, figs. 2, 2a.

Lamellaria prodita Lovén, Öfvers. Kongl. Vet.-Akad. Förh., Stockholm, 1846, vol. 3, p. 147. "FINM[ARK]."

ME.- Eastport.

Marsenina ampla Verrill.

Verrill, Proc. U. S. Nat. Mus., 1880, vol. 3, p. 374. "EAstPort, Me." Verrill, Trans. Conn. Acad., 1882, vol. 5, p. 518, pl. 42 , figs. 3 , 3a.

ME.-- Eastport.

\section{CALYPTRAEIDAE.}

*Crucibulum striatum (Say).

Gould, Inv. Mass., 1870, p. 275, fig. 536.

Calyptraea striata Say, Journ. Acad. Nat. Sci. Phila., 1826, ser. 1, vol. 5, p. 216. "NEW JERSEy, ABOUT THREE MILES NORTH OF Long Branch."

Me.- Casco Bay; Frenchman's Bay; Eastport; Isle au Haute.

Mass.- Vineyard Sound and Buzzards Bay, 3 to 12 fathoms.

ConN.- Off New London.

*Crepidula fornicata (Linné).

Gould, Inv. Mass., 1870, p. 271, fig. 532.

Patella fornicata Linné, Syst. Nat., ed. 12, 1767, vol. 1, p. 1257.

"ad Ilvam insulam, inque M. Mediterraneo." 
Common from Casco Bay, Me., to Connecticut; Frenchman's Bay, Me., rare.

\section{${ }^{*}$ Crepidula glauca Say.}

Say, Journ. Acad. Nat. Sci. Phila., 1822, ser. 1, vol. 2, p. 226. "COASt of the United States." Gould, Inv. Mass., 1870, p. 274 , fig. 535 .

ME.-Quahog Bay.

Mass.-Chelsea Beach; Vineyard Sound and Buzzards Bay. Conn.- New Haven; Stratford.

\section{${ }^{*}$ Crepidula glauca convexa Say.}

Crepidula convexa Say, Journ. Acad. Nat. Sci. Phila., 1822, ser. 1, vol. 2, p. 227. "United States."

ME. to Conn.-A form due to growing on the shells of Alectrion obsoleta and other very convex surfaces.

*Crepidula plana Say.

Say, Journ. Acad. Nat. Sci. Phila., 1822, ser. 1, vol. 2, p. 226. [New Jersey to Florida.] Gould, Inv. Mass., 1870, p. 272, fig. 533 .

Me.-Casco Bay; Old Orchard; Isle au Haute.

Mass.- Revere; Provincetown; Vineyard Sound and Buzzards Bay, 1 to 12 fathoms.

R. I.- Narragansett Bay; Watch Hill.

ConN.-Stonington; New Haven; Stratford.

\section{CAPULIDAE.}

Capulus ungaricus (Linné).

Patella ungarica Linné, Syst. Nat., ed. 12, 1767, vol. 1, p. 1259. "M. Mediterraneo."

Capulus hungaricus Tryon, Manual Conch., 1886, ser. 1, vol. 8, 
p. 131, pl. 39, figs. 72-74. Verrill, Trans. Conn. Acad., 1882, vol. 5 , p. 519.

MAss.- Off Marthas Vineyard, 69 and 458 fathoms.

\section{TRUNCATELLIDAE}

Truncatella truncata (Montagu).

Turbo truncatus Montagu, Test. Brit., 1803, pt. 2, p. 300, pl. 10, fig. 7. "Southampton, at Plymouth, and other parts of the Devonshire COAST."

Truncatella truncatula Verrill, Proc. U. S. Nat. Mus., 1880, vol. 3, p. 376; Trans. Conn. Acad., 1882, vol. 5, p. 525, pl. 58, figs. 8 , $8 \mathrm{a}, 8 \mathrm{~b}$.

R. I.- Among the docks at Newport, July, 1880. "It occurred among decaying sea-weeds thrown up at high-water mark" (Verrill).

\section{ASSIMINEIDAE.}

Assiminea modesta (H. C. Lea).

Cingula modesta H. C. Lea, Proc. Boston Soc. Nat. Hist., 1845, vol. 1, p. 205. "BRooklyn, Long Island."

Assiminea grayana Verrill, Trans. Conn. Acad., 1882, vol. 5, p. 525, pl. 58, fig. 7 (non Leach).

Assiminia modesta Verrill, Trans. Conn. Acad., 1884, vol. 6, p. 253.

R. I.-Found in July, 1880, living among decaying sea-weed at high-water mark, between the docks at Newport (Verrill).

\section{VIVIPARIDAE.}

*Campeloma decisum (Say).

Limnaea decisa Say, Nich. Encycl., 1817, pl. 3, fig. 6.

Paludina decisa Haldeman, Monogr. Limniades, 1840, pt. 1, p. 4 , pl. 1.

Melantho decisa Gould, Inv. Mass., 1870, p. 289, figs. 552-554. 
Me.-Saco River; Aroostook and Fish Rivers. Common in most of the streams and lakes, from northern MaIne to ConnectiCUT.

\section{*Campeloma rufum (Haldeman).}

Baker, Bull. Chicago Acad. Sci., 1902, no. 3, pt. 2, p. 358, pl. 36, figs. 4,6 .

Paludina rufa Haldeman, Monogr. Limniades, 1844, pt. 3, p. 3 of wrapper, pl. 3, fig. 1.

ME. - South Pond, Buckfield (J. A. Allen).

VT.-Lake Champlain.

Mass.-Pontoosuc Lake.

Conn.- Housatonic River.

\section{*Viviparus malleatus (Reeve).}

Paludina malleata Reeve, Conch. Icon., 1863, vol. 14, Paludina, pl. 5, figs. 25a, 25b. "JAPAN."

Mass.- Muddy River, between Boston and Brookline--introduced (see Nautilus, 1915, vol. 29, p. 35).

\section{VALVATIDAE.}

\section{*Valvata tricarinata (Say).}

Gould, Inv. Mass., 1870, p. 286, figs. 549, 550. Walker, Nautilus, 1902, vol. 15, p. 122, fig. 1.

Cyclostoma tricarinata Say, Journ. Acad. Nat. Sci. Phila., 1817, ser. 1, vol. 1, p. 13. " River Delaware."

ME. to ConN.- Common in most of the lakes and ponds.

*Valvata tricarinata confusa Walker.

Walker, Nautilus, 1902, vol. 15, p. 124, fig. 2. No locality.

VT.-Shelburne Bay, Lake Champlain. 
*Valvata tricarinata basalis Vanatta.

Vanatta, Nautilus, 1915, vol. 28, p. 105, figs. 3, 4. " HudsoN River, New York."

VT.- Shelburne Bay, Lake Champlain.

*Valvata tricarinata unicarinata DeKay.

Valvata unicarinata DeKay, Nat. Hist. N. Y., Moll., 1843, p. 118, pl. 6, fig. 129. "LAKE Champlain * * Erie Canal."

VT.- Shelburne Bay, Lake Champlain.

*Valvata tricarinata simplex Gould.

Gould, Inv. Mass., 1841, p. 226. "Vermont."

VT.-- Shelburne Bay, Lake Champlain.

Valvata sincera Say.

Say, Narrative Long's Exped., 1824, vol. 2, p. 264, pl. 15, fig. 11. " NORTH-WEST TERRITORY."

ME.- Dredged in the Fish River Lakes (Nylander).

VT.

*Valvata sincera nylanderi Dall.

Vralvata (sincera var.?) nylanderi Dall, Harriman Alaska Exped., 1905, vol. 13, p. 122. "Aroostook County, Maine."

Me.- Portage Lake, Fish River.

Valvata lewisi Currier.

Currier, Misc. Pub. Kent Sci. Inst., 1868, no. 1, p. 9. Dall, Harriman Alaska Exped., 1905, vol. 13, p. 123, fig. 44.

Valvata striata Lewis, Proc. Acad. Nat. Sci. Phila., 1856, p. 260 (non Philippi, 1836). [Otsego Co., N. Y.]

$\mathrm{V}_{\mathrm{T}}$. 


\section{AMNICOLIDAE.}

*Amnicola limosa (Say).

Gould, Inv. Mass., 1870, p. 293, fig. 559.

Paludina limosa Say, Journ. Acad. Nat. Sci. Phila., 1817, ser. 1, vol. 1, p. 125. " "RIVERS DELAWARE AND SchUYLKILL."

Amnicola porata Gould, Inv. Mass., 1841, p. 229, fig. 157.

ME. to Conn.- Common in most of the ponds and brooks.

Amnicola pallida Haldeman.

Haldeman, Monogr. Limniades, 1841, pt. 4, cover pp. 3, 4; 1845, pt. 8, p. 12, pl. 1, fig. 7. "Middlebury, Vermont." Gould, Inv. Mass., 1870 , p. 292, fig. 558.

ME.-Saco; South Jefferson.

VT.- Lake Champlain; Hartland.

Conn.- Hartford (Linsley); Housatonic River.

*Amnicola winkleyi Pilsbry.

Pilsbry, Nautilus, 1912, vol. 26, p. 1, pl. 1, figs. 9, 10. "SACo, Maine."

ME.- Saco. Recorded by Lermond as A. schrokingeri Ffld. N. H.- Keene.

Mass.- Ipswich (Bryant).

\section{*Lyogyrus pupoidea (Gould).}

Gill, Proc. Acad. Nat. Sci. Phila., 1863, p. 34.

Valvata pupoidea Gould, Amer. Journ. Sci., 1840, ser. 1, vol. 38, p. 196. "CAmbridge, Massachusetts." Gould, Inv. Mass., 1841 , p. 226, fig. 155 .

ME.- Monmouth; Orono.

Mass.- Fresh Pond, Cambridge; Concord; Danvers; Duxbury; Wareham.

Conn.--Housatonic and Mystic Rivers. 


\section{Lyogyrus brownii (H. F. Carpenter).}

Amnicola brownii H. F. Carpenter, Central Falls (R. I.) Weekly Visitor, 1872; Random Notes Nat. Hist., 1885, vol. 2, p. 63. " Cunliff's Pond, at Elmville, [R. I.]."

R. I.- Lakes in Roger Williams Park, and old Warwick Pond near Providence.

\section{*Lyogyrus granum (Say).}

Pilsbry, Nautilus, 1898, vol. 12, p. 42.

Paludina grana Say, Journ. Acad. Nat. Sci. Phila., 1822, ser. 1, vol. 2, p. 378. "Pennsylvania."

Amnicola granum Gould, Inv. Mass., 1870, p. 294, fig. 560.

Mass.-South Sudbury.

Conn.- New Haven (Linsley).

\section{*Somatogyrus subglobosus (Say).}

Baker, Bull. Chicago Acad. Sci., 1902, no. 3, pt. 2, p. 340, fig. 123.

Paludina subglobosa Say, Journ. Acad. Nat. Sci. Phila., 1825, ser. 1, vol. 5, p. 125. " NORTh-Western Territory."

Somatogyrus isogomus Binney, Smithson. Mise. Coll., 1865, vol. 7, art. 3, p. 77, fig. 151 .

VT.--St. Albans Bay, Lake Champlain (Dr. H. F. Perkins).

\section{*Bythinia tentaculata (Linné).}

Baker, Bull. Chicago Acad. Sci., 1902, no. 3, pt. 2, p. 328, fig. 34, figs. 116, 117 .

Helix tentaculata Linné, Syst. Nat., ed. 12, 1767, vol. 1, p. 1249. "in Europae stagnis."

Vt.-Lake Champlain (Dr. H. F. Perkins).

*Paludestrina salsa Pilsbry.

Pilsbry, Nautilus, 1905, vol. 19, p. 90, pl. 3, fig. 10, “Cohasset, Mass." 
MAss.- Cohasset (Bryant); East Wareham; 'Danvers and Rowley (Winkley).

Conn.-Branford (Winkley).

*Paludestrina minuta (Totten).

Turbo minutus Totten, Amer. Journ. Sci., 1834, ser. 1, vol. 26, p. 369, fig. 6a, b. "Mass[achusetts], and R[Hode] I[SLand]."

Rissoa minuta Gould, Inv. Mass., 1870, p. 298, fig. 566.

Littorinella minuta Verrill, Rept. Inv. Vineyard Sound, 1873, p. 653, pl. 24, fig. 140 .

ME. to ConN.- Common, especially upon the salt marshes.

Paludestrina laevis (DeKay).

Cingula laevis DeKay, Nat. Hist. N. Y., Moll., 1843, p. 111, pl. 6, fig. 118. "Stratford, (Conn.)."

Littorinella laevis Verrill, Rept. Inv. Vineyard Sound, 1873, p. 653 .

? Rissoa stimpsoni. Smith, Ann. Lyc. Nat. Hist. N. Y., 1870, vol. 9 , p. 393 .

Conn.- Stratford; Near New Haven.

*Pomatiopsis lapidaria (Say).

Gould, Inv. Mass., 1870, p. 295, figs. 561, 562.

Cyclostoma lapidaria Say, Journ. Acad. Nat. Sci. Phila., 1817, ser. 1, vol. 1, p. 13. No locality.

Mass.- Revere ? (Roper Coll.)

Conn.- Housatonic River; New Haven.

RISSOIDAE.

* Cingula carinata Mighels and Adams.

Mighels and Adams, Boston Journ. Nat. Hist., 1S42, vol. 4, p. 49.

Cingula semicostata Mighels and Adams, Boston Journ. Nat. 
Hist., 1842, vol. 4, p. 49, pl. 4, fig. 23 (non Montagu). "Casco BAY,"

Rissoa pelagica Stimpson, Proc. Boston Soc. Nat. Hist., 1851, vol. 4 , p. 15 .

Rissoa carinata Gould, Inv. Mass., 1870, p. 301, fig. 572.

ME.-Casco Bay; Frenchman's Bay, abundant in 15 fathoms (Blaney); Eastport.

*Cingula arenaria Mighels and Adams.

Mighels and Adams, Boston Journ. Nat. Hist., 1842, vol. 4, p. 49, pl. 4, fig. 24. "CAsco BAY."

Rissoa exarata Stimpson, Proc. Boston Soc. Nat. Hist., 1851, vol. 4 , p. 15 .

Rissoa mighelsi Gould, Inv. Mass., 1870, p. 301 (not fig. 570).

Me.-Casco Bay, 6 to 25 fathoms; Frenchman's Bay, 6 to 8 fathoms; Eastport.

Mass.- Boston Harbor, 3 fathoms.

R. I.-Watch Hill, 4 to 5 fathoms, among rocks and algae (Verrill).

Conn.-Stonington, from cod (Linsley).

*Cingula areolata (Stimpson).

Verrill, Trans. Conn. Acad., 1882, vol. 5, p. 524, pl. 43, fig. 2.

Turritelle arcolata Stimpson, Shells of New England, 1851, p. 35. "Mass[achusetts] Bay."

ME.-Frenchman's Bay, 6 to $S$ fathoms (Blaney); Mt. Desent, 10 to 15 fathoms (Verrill).

Mass.-Massachusetts Bay; off Marthas Vineyard, 130 fathoms.

* Cingula castanea (Möller).

G. O. Sars, Moll. Reg. Arct. Norv., 1878, p. 174, pl. 10, figs. 1a, $1 \mathrm{~b}$. 
Rissoa castanea Möller, Kroyer's Naturh. Tidskr., 1842, vol. 4, p. 82. [Greenland.]

ME.-Frenchman's Bay, 6 to 12 fathoms; Eastport; Penobscot Bay.

Cingula latior Mighels and Adams.

Mighels and Adams, Boston Journ. Nat. Hist., 1842, vol. 4, p. 48, pl. 4, fig. 22. "CAsco BAY."

Rissoa latior Gould, Inv. Mass., 1870, p. 299, fig. 567.

ME.- Casco Bay, from the stomach of a haddock.

\section{Cingula harpa (Verrill).}

Verrill, Trans. Conn. Acad., 1882, vol. 5, p. 523, pl. 58, fig. 6.

Rissoa (Cingula) harpa Verrill, Proc. U. S. Nat. Mus., 1880, vol. 3, p. 374. " off Massachusetrs Bay.”

Mass.- Off Massachusetts Bay, 160 fathoms; off Marthas Vineyard, 312 to 487 fathoms.

\section{*Cingula multilineata (Stimpson).}

Rissoa multilineata Stimpson, Proc. Boston Soc. Nat. Hist., 1851, vol. 4, p. 14. "Gt. Misery Island, and also near Nahant, [Mass.]." Gould, Inv. Mass., 1870, p. 300, fig. 569.

ME.-Frenchman's Bay, 5 fathoms (Blaney).

MAss.-Off Great Misery Id., 5 fathoms; near Nahant; Provincetown.

*Onoba aculeus (Gould).

Cingula aculeus Gould, Inv. Mass., 1841, p. 266, fig. 172. “ EAST Boston."

Rissoa aculeus Gould, Inv. Mass., 1870, p. 299, fig. 568.

ME.-Casco Bay; Frenchman's Bay; Eastport. 
Mass.- Massachusetts Bay; Vineyard Sound; Duxbury; Provincetown.

R. I.- Watch Hill.

Conn.-New Haven; Stratford.

LITIOPIDAE.

\section{${ }^{*}$ Litiopa bombix Kiener.}

Kiener, Ann. des Sci. Nat., 1833, vol. 30, p. 222, pl. 17, B, figs. 1-3. No locality. Verrill, Trans. Conn. Acad., 1882, vol. 5, p. 523 .

Litiopa melanostoma Sumner, Osburn, and Cole, Bull. U. S. Bureau Fisheries, 1913, vol. 31, pt. 2, p. 720.

Mass.-Off Marthas Vineyard. Pelagic, found attached to floating gulfweed (Sargassum sp.).

\section{HOMALOGYRIDAE.}

${ }^{*}$ Homalogyra atomus (Philippi).

Jeffreys, Brit. Conch., 1867, vol. 4, p. 69, pl. 1, fig. 5.

Truncatella atomus Philippi, Arch. f. Naturg., 1841, vol. 7, pt. 1, p. 54, pl. 5, fig. 4. "in Sorrent [Italy]."

N. H.- Hampton Beach (Morse).

\section{SKENEIDAE.}

*Skenea planorbis (Fabricius).

Gould, Inv. Mass., 1870, p. 296, fig. 563.

Turbo planorbis Fabricius, Fauna Groenlandica, 1780, p. 394. [GREENLAND.]

ME.- Common, Casco Bay to Eastport.

Mass.-Cape Ann; Provincetown; Cohasset; Woods Hole.

R. I.- Watch Hill.

Conn.- Near New Haven. 
Adeorbis supranitidus orbignyi Fischer.

Dall, Bull. Mus. Comp. Zoöl., 1889, vol. 18, p. 278.

Adeorbis orbignyi Fischer, Journ. de Conch., 1857, vol. 6, pp. 173, 286. "Cuba."

Omalaxis? lirata Verrill, Trans. Conn. Acad., 1882, vol. 5, p. 529.

R. I.- Off Newport, $8 \frac{1}{4}$ fathoms.

Adeorbis? olivaceus (Verrill).

Dall, Bull. U. S. Nat. Mus., 1889, no. 37, p. 150, pl. 44, fig. 5.

Cithna (?) olivacea Verrill, Trans. Conn. Acad., 1884, vol. 6, p. 185, pl. 29, fig. 5. “ off Martha's Vineyard.”

Mass.-- Off Marthas Vineyard, 193 fathoms, and 1290 fathoms.

SOLARIIDAE.

Architectonica borealis (Verrill and Smith).

Solarium boreale Verrill and Smith in Verrill, Proc. U. S. Nat. Mus., 1880, vol. 3, p. 376. [south of Marthas Vineyard.] Verrill, Trans. Conn. Acad., 1882, vol. 5, p. 529, pl. 57, figs. 29, 30.

Mass.- South of Marthas Vineyard, 115 and 146 fathoms.

$$
\text { FOSSARIDAE. }
$$

Fossarus elegans Verrill and Smith.

Verrill and Smith in Verrill, Trans. Conn. Acad., 1882, vol. 5, p. 522, pl. 57, fig. 28. "off Martha's Vineyard."

Mass.- South of Marthas Vineyard, 100 fathoms.

\section{LITORINIDAE.}

*Litorina littorea (Linné).

Turbo littoreus Linné, Syst. Nat., ed. 10, 1758, vol. 1, p. 761. "O. Europaeo." 
Littorina litorea Gould, Inv. Mass., 1870, p. 308, fig. 577.

ME. to Conn.-This species, now common along the entire coast, first appeared on the coast of Maine in 1871.

\section{*Litorina obtusata palliata (Say).}

Turbo palliatus Say, Journ. Acad. Nat. Sci. Phila., 1822, ser. 1, vol. 2, p. 240. " New England."

Littorina palliata Gould, Inv. Mass., 1870, p. 309, fig. 578.

Littorina obtusata palliata Dautzenberg and Fischer, Journ. de Conch., 1915, vol. 62, p. 107, pl. 2, fig. 4 .

ME. to Conn.- Common on the rocks between tides.

${ }^{*}$ Litorina rudis (Donovan).

Turbo rudis Donovan, Brit. Shells, 1804, vol. 1, pl. 33, fig. 3 and explanation. "Devonshire, [England]."

Littorina rudis Gould, Inv. Mass., 1870, p. 304, fig. 575.

ME. to Conn.-Common on the rocks of the ocean shore.

*Litorina rudis tenebrosa (Montagu).

Turbo tenebrosus Montagu, Test. Brit., 1803, p. 303; Suppl., 1808, pl. 20, fig. 4. "Devonshire *** Kent [England]."

Littorina tenebrosa Gould, Inv. Mass., 1870, p. 306, fig. 576.

Me. to Conn.-- Common about wharves, bridges and upon the salt marshes.

Litorina irrorata (Say).

Turbo irroratus Say, Journ. Acad. Nat. Sci. Phila., 1822, ser. 1, vol. 2, p. 239. [NEW JeRSEY to Florida.]

Littorina irrorata Gould, Inv. Mass., 1870, p. 311, fig. 579.

MASS.- Vineyard Sound.

*Lacuna vincta (Montagu).

Gould, Inv. Mass., 1841, p. 262, fig. 168. 
Turbo vinctus Montagu., Test. Brit., 1803, p. 307; Suppl., 1808, pl. 20, fig. 3. " Salcomb bay [England]."

Me. to Conn.- Common among algae in 2 to 5 fathoms.

\section{*Lacuna vincta fusca Gould.}

Gould, Inv. Mass., 1841, p. 263, fig. 169. [Massachusetts.]

ME. to Conn.- Associated with the typical form.

*Lacuna puteola (Turton).

Turbo puteolus Turton, Conch. Dictionary, 1819, p. 193, figs. 90, 91. "Dublin bay, and on the rocks between Teignmouth and Dawlish, in Devonshire, attached to Fuci."

Lacuna puteolus Forbes and Hanley, Hist. Brit. Moll., 1853, vol. 3, p. 58, pl. 72, figs. 7-9; pl. 74, fig. 9. Tryon, Manual Conch., 1887 , ser. 1 , vol. 9 , p. 265 , pl. 50, figs. $51,52$.

Mass.-Vineyard Sound; Buzzards Bay near the Elizabeth Islands; Nantucket.

*Lacuna pallidula neritoidea Gould.

Lacuna neritoidea Gould, Amer. Journ. Sci., 1840, vol. 38, p. 197. [Chelsea Beach, Mass.] Gould, Inv. Mass., 1870, p. 303, fig. 574.

Me.-Casco Bay; Cape Elizabeth; Eastport.

Mass.-Swampscott; Revere; Duxbury.

Conn.-Oyster River; Long Beach; Stratford (Linsley). These records are questioned by Prof. Verrill.

TURRITELLIDAE.

*Turritella erosa Couthouy.

Couthouy, Boston Journ. Nat. Hist., 1838, vol. 2, p. 103, pl. 3, fig. 1. "Mass[achusetts] Bay." Gould, Inv. Mass., 1870, p. 317 , fig. 585 . 
Me.-Casco Bay; Frenchman's Bay, 7 fathoms; near Eastport, 50 fathoms.

Mass.-Ipswich Bay; Massachusetts Bay.

Conn.- From stomach of a cod off Stonington (Linsley).

\section{*Turritellopsis acicula (Stimpson).}

G. O. Sars, Moll. Reg. Arct. Norv., 1878, p. 186, pl. 10, figs. 14a, 14b.

Turritella acicula Stimpson, Proc. Boston Soc. Nat. Hist., 1851, vol. 4, p. 15. "From fishes CAUGHT OFF *** CAPE ANN, [MAss.]." Gould, Inv. Mass., 1870, p. 319, fig. 588.

Me.-Casco Bay; Frenchman's Bay, 5 to 10 fathoms; near Eastport, 40 fathoms.

Mass.- From fish caught off Cape Ann; Lynn and Marshfield; Duxbury.

\section{VERMETIDAE,}

*Vermicularia spirata (Philippi).

Vermetus spiratus Philippi, Arch. f. Naturg., 1836, vol. 2, pt. 1, p. 224, pl. 7, fig. 1. " ad Havanam, [Cuba]."

Vermetus radicula Stimpson, Shells of New England, 1851, p. 37. Gould, Inv. Mass., 1870, p. 316, fig. 584.

Mass.- Vineyard Sound and Buzzards Bay, 3 to 10 fathoms; New Bedford Harbor.

Conn.-- New Haven (Linsley).

\section{CAECIDAE.}

*Caecum pulchellum Stimpson.

Stimpson, Proc. Boston Soc. Nat. Hist., 1851, vol. 4, p. 112. “Buzzard's Bay [, Mass.]." Gould, Inv. Mass., 1870, p. 315, fig. 583 .

N. H.- Hampton Beach (Morse).

Mass.- Vineyard Sound, 1 to 4 fathoms; New Bedford Harbor. 
${ }^{*}$ Caecum cooperi S. Smith.

S. Smith, Ann. Lyc. Nat. Hist. N. Y., 1860, vol. 7, p. 154 (non Carpenter, 1864). "Gardiner's Bay, [L. I.]."

Caecum costatum Verrill, Amer. Journ. Sci., 1872, ser. 3, vol. 3, p. 283 , pl. 6 , fig. 6 .

Mass.- Vineyard Sound, 8 to 10 fathoms.

*Caecum johnsoni Winkley.

Winkley, Nautilus, 1908, vol. 22, p. 54. "Woods Hole, Mass." Mass.-Woods Hole.

\section{TRICHO'TROPIDAE.}

*Trichotropis borealis Broderip and Sowerby.

Broderip and Sowerby, Zool. Journ., 1828, vol. 4, p. 375. " oceano boreali prope Insulam Melville." Gould, Inv. Mass., 1870, p. 390, fig. 651.

Trichotropis costellatus Couthouy, Boston Journ. Nat. Hist., 1838, vol. 2, p. 108, pl. 3, fig. 2.

Me.-Casco Bay; Frenchman's Bay, 25 to 50 fathoms; Eastport, 20 fathoms.

Mass.- Deep waters of Massachusetts Bay (Couthouy).

Trichotropis conica Möller.

Möller, Krøyer's Naturh. 'Tidskr., 1842, vol. 4, p. 85. [GreenLANd.] G. O. Sars, Moll. Reg. Arct. Norv., 1878, p. 163, pl. 13, fig. 3. Verrill, Proc. U. S. Nat. Mus., 1880, vol. 3, p. 374.

Gulf of Maine, off Cape Sable, 75 fathoms.

Torellia fimbriata Verrill and Smith.

Verrill and Smith in Verrill, Trans. Conn. Acad., 1882, vol. 5, p. 520 , pl. 57 , figs. 27,27 a. [off the coast of Maine and south of Marthas Vineyard.] 
Gulf of Maine, near Cashe's Ledge, 52 to 90 fathoms.

MASs.- South of Marthas Vineyard, 142 to 258 fathoms.

Torellia vestita Jeffreys.

Jeffreys, Brit. Conch., 1867, vol. 4, p. 244, pl. 4, fig. 1. “EAST coast of Shetland (Barlee).” Verrill, Trans. Conn. Acad., 1882, vol. 5, p. 521, pl. 47, fig. 5 .

Gulf of Maine, 150 fathoms.

MASS.- South of Marthas Vineyard, 86 to 146 fathoms.

\section{PLEUROCERIDAE.}

${ }^{*}$ Goniobasis virginica (Gmelin).

Tryon, Smithson. Misc. Coll., 1873, vol. 16, art. 1, p. 290, figs. 562-566.

Buccinum virginicum Gmelin, Syst. Nat., 1790, vol. 1, pt. 6, p. 3505. "IN Virginiae fluvirs."

Melania virginica Say, Amer. Conch., 1832, pt. 5, pl. 47, fig. 2 and text.

MAss.-Connecticut River, at Agawam, below Springfield (Winkley).

Conn.- Connecticut River, at Deep River (Reid).

*Goniobasis virginica multilineata (Say).

Melania multilineata Say, Journ. Acad. Nat. Sci. Phila., 1822, ser. 1, vol. 2, p. 380. " tributaries to the Delaware."

Goniobasis virginiea Tryon, Smithson. Mise. Coll., 1873, vol. 16, art. 1, p. 290, fig. 567 .

Conn.-- Connecticut River, at Deep River (Reid).

Goniobasis depygis (Say).

Tryon, Smithson. Mise. Coll., 1873, vol. 16, art. 1, p. 247, figs. 481, 482. 
Melania depygis Say, New Harmony (Ind.) Disseminator, 1829, p. 291. [FALL OF THE OHIO.]

Vт.-Lake Champlain.

*Goniobasis haldemani Tryon.

Tryon, Amer. Journ. Conch., 1865, vol. 1, p. 38, pl. 1, fig. 8. "Lake Erie, Lake Champlain." Tryon, Smithson. Misc. Coll., 1873, vol. 16, art. 1, p. 282.

VT.-Lake Champlain.

TRIPHORIIDAE.

*Triphoris perversa nigrocincta (C. B. Adams).

Cerithium nigrocinctum C. B. Adams, Boston Journ. Nat. Hist., 1839, vol. 2, p. 286, pl. 4, fig. 11. "Dartiouth harbor, [Mass.]."

Triforis nigrocinctus Gould, Inv. Mass., 1870, p. 323, fig. 592.

Mass.-- Vineyard Sound and Buzzards Bay, low water to 10 fathoms; Wellfleet.

R. I.-- Narragansett Bay; Watch Hill.

ConN.-- Stonington on oysters; near New Haven.

\section{CERITHIOPSIDAE.}

${ }^{*}$ Cerithiopsis greenii (C. B. Adams).

Verrill, Rept. Inv. Vineyard Sound, 1873, p. 647, pl. 24, fig. 153.

Cerithium greenii C. B. Adams, Boston Journ. Nat. Hist., 1839, vol. 2, p. 287, pl. 4, fig. 12. "Dartmouth harbor, [Mass.]."

Bittium greenii Gould, Inv. Mass., 1870, p. 322, fig. 591.

MAss.- Boston Harbor; Vineyard Sound and Buzzards Bay, 3 to 10 fathoms.

R. I.- Narragansett Bay; Watch Hill.

Conn.-- Near New Haven (Perkins).

${ }^{*}$ Cerithiopsis subulata (Montagu).

Murex subulatus Montagu, Test. Brit., Suppl., 1808, p. 115, pl. 30, fig. 6. " at Scalasdale, in the sound of Mull." 
Cerithiopsis emersonii Gould, Inv. Mass., 1870, p. 387, fig. 649. Cerithiopsis (Eumeta?) subulata Dall, Bull. Mus. Comp. Zoöl., 1889, vol. 18, p. 252, pl. 20, fig. 4 .

Mass.- Vineyard Sound and Buzzards Bay, 2 to 12 fathoms; New Bedford Harbor; Nantucket; Wellfleet.

R. I.

\section{Cerithiopsis costulata (Möller).}

G. O. Sars, Moll. Reg. Arct. Norv., 1878, p. 189, pl. 13, fig. 7.

Turritella ? costulata Möller, Krøyer's Naturh. Tidskr., 1842, vol. 4, p. 83. [GreenLAND.]

Me.- Off Eastport, Bay of Fundy (Verrill).

\section{*Seila adamsii (H. C. Lea).}

Dall, Trans. Wagner Free Inst. Sci., 1892, vol. 3, pt. 2, p. 267.

Cerithium terebrale C. B. Adams, Boston Journ. Nat. Hist., 1840, vol. 3, p. 320, pl. 3, fig. 7 (non Lamarck). “ NEW BEDFORD [, Mass.]."

Cerithium adamsii H. C. Lea, Trans. Amer. Phil. Soc., 1846, new ser., vol. 9, p. 268.

Cerithiopsis terebralis Gould, Inv. Mass., 1870, p. 389, fig. 650.

Mass.- Vineyard Sound and Buzzards Bay, 2 to 12 fathoms.

R. I.- Narragansett Bay.

Conn.

\section{CERITHIIDAE.}

${ }^{*}$ Bittium alternatum (Say).

Turritella alternata Say, Journ. Acad. Nat. Sci. Phila., 1822, ser. 1, vol. 2, p. 243. "United States."

Pasithea nigra Totten, Amer. Journ. Sci., 1834, ser. 1, vol. 26, p. 369 , pl. 1, figs. 7a, $7 \mathrm{~b}$ (young).

Bittium nigrum Gould, Inv. Mass., 1870, p. 321, fig. 590. 
Mass.-Boston Harbor; Vineyard Sound and Buzzards Bay, low water to 8 fathoms, among algae.

R. I.- Narragansett Bay.

Conn.-New Haven.

\section{APORRIAIDAE.}

*Aporrhais (Arrhoges) occidentalis (Beck).

Gabb, Amer. Journ. Conch., 1868, vol. 4, p. 145.

Rostellaria occidentalis Beck, Mag. de Zool., 1836, vol. 6, classe 5, pl. 72 and text. [Gulf of St. Lawrence and Newfoundland.] Aporrhais occidentalis Gould, Inv. Mass., 1870, p. 320, fig. 589.

ME.-Casco Bay; Frenchman's Bay, 10 to 35 fathoms; Eastport, 20 fathoms.

Mass.- Nahant Beach; off Duxbury.

\section{DOLIIDAE.}

Eudolium crosseanum (Monterosato).

Dolium crosseanum Monterosato, Journ. de Conch., 1869, vol. 17, p. 228, pl. 12, fig. 1. [off Palermo, Italy.]

Dolium bairdii Verrill and Smith in Verrill, Amer. Journ. Sci., 1881, ser. 3, vol. 22, p. 299. Verrill, Trans. Conn. Acad., 1882, vol. 5 , p. 515 ; 1884 , vol. 6 , p. 253 , pl. 29 , figs. $2,2 \mathrm{a}, 2$ b.

Dolium (Eudolium) crosseanum Dall, Bull. U. S. Nat. Mus., 1889, no. 37 , p. 134 , pl. 15 , fig. 5 ; pl. 44 , figs. $2-2$ b; pl. 62 , figs. 83, 83a.

Mass.-Off Marthas Vineyard, 94 to 202 fathoms.

PTEROTRACHEIDAE.

Firoloida lesueurii (d'Orbigny).

E. A. Smith, Rept. Voy. Challenger, Zool., 1888, vol. 23, art. 5, p. 23. Verrill, Trans. Conn. Acad., 1885, vol. 6, p. 430. 
Firola (Cerophora) lesueurii d'Orbigny, Voy. Amér. Mérid., 1836, vol. 5, p. 151, pl. 10, figs. 11, 12 . [Océan Austral, S. LAT. $30^{\circ}$, W. LONG. $89^{\circ}$.]

Mass.-South of Marthas Vineyard, N. lat. $39^{\circ} 43^{\prime}$, W. long. $70^{\circ} 07^{\prime}$, surface.

\section{CARINARIIDAE.}

Carinaria atlantica Adams and Reeve.

Adams and Reeve, Zool. Voy. Samarang, Moll., 1848, no. 3, p. 63, pl. 13, fig. 12. " North Atlantic Ocean." Verrill, Trans. Conn. Acad., 1882, vol. 5, p. 529; 1884, vol. 6, p. 274.

MAss.-Off Marthas Vineyard, N. lat. $40^{\circ} 05^{\prime}$, W. long. $70^{\circ} 23^{\prime}$, 65 fathoms.

\section{ATLANTIDAE.}

\section{*Atlanta peronii Lesueur.}

Lesueur, Journ. de Physique, 1817, vol. 85, p. 390, pl. 2, figs. 1, 2. Verrill, Trans. Conn. Acad., 1884, vol. 6, p. 274, pl. 28, figs. 4, 4a.

Atalanta peronii Verrill, Trans. Conn. Acad., 1882, vol. 5, p. 529.

Near the Georges Bank, N. lat. $41^{\circ} 25^{\prime}$, W. long. $65^{\circ} 30^{\prime}$, surface.

Atlanta gaudichaudii Eydoux and Souleyet.

Eydoux and Souleyet, Voy. Bonité, Zoöl., 1852, vol. 2, p. 379; atlas, pl. 19, figs. 29-34. "PAcifique." Verrill, Trans. Conn. Acad., 1884, vol. 6, pp. 211, 274.

Off the Georges Bank, N. lat. $40^{\circ} 02^{\prime} 49^{\prime \prime}$, W. long. $68^{\circ} 40^{\prime}$, surface.

Atlanta rosea Eydoux and Souleyet.

Eydoux and Souleyet, Voy. Bonité, Zoöl., 1852, vol. 2, p. 377; atlas, pl. 19, figs. 16-20. “Atrantique." Verrill, Trans. Conn. Acad., 1884, vol. 6, p. 211.

Off the Georges Bank, N. lat. $40^{\circ} 16^{\prime} 15^{\prime \prime}$, W. long. $67^{\circ} 05^{\prime} 15^{\prime \prime}$, surface. 
Atlanta inclinata Eydoux and Souleyet.

Eydoux and Souleyet, Voy. Bonité, Zoöl., 1852, vol. 2, p. 375; atlas, pl. 19, figs. 9-15. "Pacifique et Atlantique." Verrill, Trans. Conn. Acad., 1884, vol. 6, p. 211.

Off Georges Bank, N. lat. $40^{\circ} 16^{\prime} 50^{\prime \prime}$, W. long. $67^{\circ} 05^{\prime} 15^{\prime \prime}$, surface.

Atlanta pulchella Verrill.

Verrill, Trans. Conn. Acad., 1884, vol. 6, p. 211. "off DelAWARE BAY, N. LAT. $39^{\circ} 22^{\prime}$, W. LONG. $68^{\circ} 34^{\prime} 30^{\prime \prime}$."

Mass.-Off Marthas Vineyard, N. lat. $40^{\circ}$, surface.

\section{Oxygyrus keraudrenii (Lesueur).}

H. and A. Adams, Genera Recent Moll., 1858, vol. 2, p. 92; vol. 3, pl. 69, figs. 6-6b. Verrill, Trans. Conn. Acad., 1885, vol. 6, p. 430.

Atlanta keraudrenii Lesueur, Journ. de Physique, 1817, vol. 85, p. 391, pl. 2 .

Mass.-South of Marthas Vineyard, N. lat. $39^{\circ} 44^{\prime}$, W. long. $70^{\circ} 03^{\prime}$, surface.

\section{MURICIDAE.}

*Eupleura caudata (Say).

Stimpson, Amer. Journ. Conch., 1865, vol. 1, p. 58, pl. 8, fig. 5.

Ranella caudata Say, Journ. Acad. Nat. Sci. Phila., 1S22, ser. 1, vol. 2, p. 236. " COASt of the United States." Gould, Inv. Mass., 1870, p. 38̣6, fig. 648.

Mass.-- Vineyard Sound, 1 to 8 fathoms; Buzzards Bay; Nantucket; Marthas Vineyard.

R. I.-Coweset Bay; Nayatt.

Conn.-Off New Haven, 1 to 5 fathoms; Long Beach; Stratford. 


\section{*Trophon clathratus (Linné).}

Murex clathratus Linné, Syst. Nat., ed. 12, 1767, vol. 1, p. 1223. "In Istandiae Mart."

Trophon scalariformis Gould, Inv. Mass., 1870, p. 378, fig. 644.

Me.-Casco Bay; Eastport, 20 fathoms.

Mass.-From a fish caught in Massachusetts Bay.

\section{Trophon clathratus gunneri (Lovén).}

Verrill, Trans. Conn. Acad., 1882, vol. 5, p. 512, pl. 43, fig. 8.

Tritonium gunneri Lovén, Öfvers. Kongl. Vet.-Akad. Förh. Stockholm, 1846, vol. 3, p. 144. "NORdL[ICHE] Finm[ARK.]"

Gulf of Maine, off Grand Manan; off Cape Sable, 59 fathoms.

\section{Trophon truncatus (Ström).}

Buccinum truncatum Ström, Norske Vidensk. Selsk. Skr., 1767, vol. 4, p. 369, pl. 16, fig. 26.

Fusus bamffius Gould, Inv. Mass., 1841, p. 289, fig. 198.

Trophon clathratus Gould, Inv. Mass., 1870, p. 377, fig. 643 (non Linné).

Me.- Casco Bay; Frenchman's Bay, 35 to 50 fathoms; Eastport, 20 fathoms.

\section{Trophon muricatus (Montagu).}

Gould, Inv. Mass., 1870, p. 379.

Murex muricatus Montagu, Test. Brit., 1803, p. 262, pl. 9, fig. 2. "Salcomb bay, [England]."

Mass-- Phillips Beach.

Trophon lintoni Verrill and Smith.

Verrill and Smith in Verrill, Amer. Journ. Sci., 1882, ser. 3, vol. 24, p. 365. [off Marthas Vineyard.] Verrill, Trans. Conn. Acad., 1884, vol. 6, p. 176, pl. 29, fig. 1.

Mass.-Off Marthas Vineyard, 70 fathoms. 


\section{*Urosalpinx cinerea (Say).}

Stimpson, Amer. Journ. Conch., 1865, vol. 1, p. 58, pl. 8, figs. 6, 7. Fusus cinereus Say, Journ. Acad. Nat. Sci. Phila., 1822, ser. 1, vol. 2, p. 236. [Maryland and New Jersey.]

Buccinum cinereum Gould, Inv. Mass., 1870, p. 370, fig. 637.

ME.-Quahog Bay; Casco Bay.

Mass.-Lynn; Cohasset; Vineyard Sound and Buzzards Bay, from above low water to 8 fathoms; Nantucket.

R. I.- Narragansett Bay; Watch Hill.

Conn.- New Haven; Stratford.

*Thais (Nucella) lapillus (Linné).

Dall, Prof. Paper U. S. Geol. Survey, 1909, no. 59, p. 50.

Buccinum lapillus Linné, Syst. Nat., ed. 10, 1758, vol. 1, p. 739. "ad Oceani Europaei littora."

Purpura lapillus Gould, Inv. Mass., 1870, p. 360, fig. 630.

ME. to Conn.- Common on the rocky shores.

\section{COLUMBELLIDAE.}

*Columbella (Anachis) avara Say.

Colombella avara Say, Journ. Acad. Nat. Sci. Phila., 1822, ser. 1, vol. 2, p. 230. " COAST OF THE SOUthern states."

Anachis arara Verrill, Rept. Inv. Vineyard Sound, 1873, p. 643.

Mass. - Woods Hole; Vineyard Sound, low water to 10 fathoms; Duxbury; Wellfleet.

R. I.- Narragansett Bay.

Cons.-New Haven.

*Columbella (Anachis) avara similis Ravenel.

Columbella similis Ravenel, Proc. Acad. Nat. Sci. Phila., 1861, p. 41. "North and South Carolina."

Columbella avara Gould, Inv. Mass., 1870, p. 356, fig. 626. 
Mass.-Massachusetts Bay; Wellfleet; Plymouth; Woods Hole; Nantucket; Marthas Vineyard.

R. I.- Narragansett Bay.

Conn.--New Haven; Stratford.

Columbella (Anachis) haliaeeti Jeffreys.

Columbella haliaceti Jeffreys, Brit. Conch., 1867, vol. 4, p. 356; 1869, vol. 5, p. 219, fig. 3. “IN 85-95 F. ABOUt 25 MILES N.N.W. of Unst."

Anachis costulata Verrill, Trans. Conn. Acad., 1882, vol. 5, p. 513, pl. 43, fig. 7 (non Cantraine).

ME.-Gulf of Maine, at Cashe's Ledge, Jeffrey Bank, etc., 30 to 114 fathoms.

Mass.-Off Cape Cod, 67 to 96 fathoms; south of Marthas Vineyard, common in 146 to 506 fathoms (Verrill).

*Columbella (Astyris) Iunata (Say).

Nassa lunata Say, Journ. Acad. Nat. Sci. Phila., 1826, ser. 1, vol. 5, p. 213. "Southern States."

Buccinum zonalis Linsley, Amer. Journ. Sci., 1845, ser. 1, vol. 48, p. 285. Gould, Amer. Journ. Sci., 1848, ser. 2, vol. 6, p. 236, fig. 8 . Columbella lunata Gould, Inv. Mass., 1870, p. 359, fig. 629.

ME.-Quahog Bay; Orr's Isle; Damariscotta (Winkley).

Mass.-Swampscott; Provincetown; Vineyard Sound and Buzzards Bay, 1 to 10 fathoms.

R. I.- Narragansett Bay.

Conn.- New Haven; Stratford.

*Columbella (Astyris) dissimilis Stimpson.

Columbella dissimilis Stimpson, Proc. Boston Soc. Nat. Hist., 1851, vol. 4, p. 114. "BAY of Fundy."

ME.- Eastport Harbor; Penobscot Bay. 
${ }^{*}$ Columbella (Astyris) rosacea (Gould).

Buccinum rosaceum Gould, Amer. Journ. Sci., 1840, ser. 1, vol. 38, p. 197. [from fish off Cohasset, Mass.]

Columbella rosacea Gould, Inv. Mass., 1870, p. 357, fig. 627.

Me.- Casco Bay, 10 to 20 fathoms; Frenchman's Bay, 6 to 35 fathoms; Eastport.

N. H.- Isles of Shoals, 20 fathoms.

Mass.-Massachusetts Bay, 15 to 25 fathoms; off Chatham, 16 fathoms.

R. I.-East of Block Island, 29 fathoms.

ConN.- Stonington.

Columbella (Astyris) diaphana (Verrill).

Astyris diaphana Verrill, Trans. Conn. Acad., 1882, vol. 5, p. 513, pl. 58, fig. 2. “off Martha's Vineyard."

Mass.- Off Marthas Vineyard, 65 to 487 fathoms.

Columbella (Astyris) pura (Verrill).

Astyris pura Verrill, Trans. Conn. Acad., 1882, vol. 5, p. 515. "off Martha's Vineyard."

Mass.-Off Marthas Vineyard, 100 to 487 fathoms.

\section{ALECTRIONIDAE}

*Alectrion (Hima) vibex (Say).

Nassa vibex Say, Journ. Acad. Nat. Sci. Phila., 1822, ser. 1, vol. 2, p. 231. "SOUTHERN AND MIDDLE COAST [OF THE UNited States].” Gould, Inv. Mass., 1870, p. 365, fig. 633.

Nassa fretensis Perkins, Proc. Boston Soc. Nat. Hist., 1869, vol. 13 , p. 117, fig.

Mass.-Vineyard Sound; New Bedford; Cataumet.

R. I.- Coweset Bay.

Conn.-- New Haven. 
Alectrion (Hima?) nigrolabra (Verrill).

Nassa nigrolabra Verrill, Proc. U. S. Nat. Mus., 1880, vol. 3, p. 371. [sovth of Marthas Vineyard.] Verrill, Trans. Conn. Acad., 1882, vol. 5, p. 512, pl. 58, fig. 12.

Mass.- South of Marthas Vineyard, 155 fathoms.

\section{*Alectrion (Ilyanassa) obsoleta (Say).}

Nassa obsoleta Say, Journ. Acad. Nat. Sci. Phila., 1822, ser. 1, vol. 2, p. 232. "COAst of the United States." Gould, Inv. Mass., 1870, p. 362, fig. 631.

Common from the mouth of the Kennebec River, Me., southward, on mud flats.

*Alectrion (Tritia) trivittata (Say).

Nassa trivittata Say, Journ. Acad. Nat. Sci. Phila., 1822, ser. 1, vol. 2, p. 231. "MiddLe AND southern states." Gould, Inv. Mass., 1870, p. 364, fig. 632.

Common from Eastport, Me., southward, in from 1 to 40 fathoms.

\section{BUCCINIDAE.}

*Buccinum undatum Linné.

WHELK.

Linné, Syst. Nat., ed. 12, 1767, vol. 1, p. 1204. "O. EuropaEo." Gould, Inv. Mass., 1870, p. 366, fig. 634.

ME.-Casco Bay; Frenchman's Bay; Eastport, common.

Mass.--Massachusetts Bay; off Gay Head, 6 to 19 fathoms.

R. I.- Rocks at Newport (Carpenter).

Conn.- Stonington, taken in eel-pots (Linsley).

Buccinum cyaneum Bruguière.

Bruguière, Encycl. Méth., Hist. Nat. des Vers, 1792, vol. 1, p. 266. " Côtes du Groenland." Verrill, Trans. Conn. Acad., 1882, vol. 5, p. 492 , pl. 43 , fig. 5 . 
Gulf of Maine, off Cape Sable, 82 to 91 fathoms.

About 15 miles east of Cape Cod, 70 to 90 fathoms.

Buccinum cyaneum perdix (Beck).

Verrill, 'Trans. Conn. Acad., 1882, vol. 5, p. 492.

Tritonium groenlandicum var. perdix Beck in Mörch, Faun. Moll. Islandiae, 1868, p. 211. [ICELAND.]

About 15 miles east of Cape Cod, 70 to 90 fathoms.

\section{Buccinum tenue Gray.}

Gray, Zool. Beechey’s Voy., 1839, p. 128, pl. 36, fig. 19. “ICY CAPE." Verrill, Trans. Conn. Acad., 1882, vol. 5, p. 495, pl. 43, fig. 4 .

Gulf of Maine, off Cape Sable, 88 to 91 fathoms.

Buccinum sandersoni Verrill.

Verrill, Trans. Conn. Acad., 1882, vol. 5, p. 490, pl. 58, fig. 9. "ofF Martha's Vineyard."

Mass.-Off Marthas Vineyard, 156, 208, and 258 fathoms.

*Neptunea decemcostata (Say).

Fusus 10-costatus Say, Journ. Acad. Nat. Sci. Phila., 1826, ser. 1, vol. 5, p. 214. " near Boston [, Mass.]." Gould, Inv. Mass., 1870, p. 375, fig. 642 .

Me.-Casco Bay; Penobscot Bay; Frenchman's Bay; Eastport.

Mass.-Off Nahant, 17 fathoms; off Cape Cod, 35 fathoms; Duxbury.

*Neptunea despecta tornata (Gould).

Fusus tornatus Gould, Amer. Journ. Sci., 1840, ser. 1, vol. 38, p. 197. [BANK Fisheries.] Gould, Inv. Mass., 1841, p. 286, fig. 201 ; 1870, p. 374, fig. 641 . 
Neptunea despecta vár. tornata Verrill, Trans. Conn. Acad., 1882, vol. 5, p. 499.

Off Georges Bank, 69 to 100 fathoms (Verrill).

*Colus stimpsonii (Mörch).

Fusus stimpsonii Mörch, Vid. Medd. Naturh. Foren. Kjöbenhavn, 1868, p. 83. "FAERö."

Fusus islandicus Gould, Inv. Mass., 1870, p. 371, fig. 638 (non Linné).

Neptunea curta Verrill, Rept. Inv. Vineyard Sound, 1873, p. 638.

Sipho stimpsonii Verrill, Trans. Conn. Acad., 1882, vol. 5, p. 499.

Me.-Casco Bay; Frenchman's Bay; Eastport.

Mass.-Ipswich Beach; Phillips Beach; Provincetown; Duxbury.

Conn.- Stonington, from fish stomachs (Linsley).

Colus stimpsonii liratulus (Verrill).

Sipho stimpsonii var. liratulus Verrill, Trans. Conn. Acad., 1882, vol. 5 , p. 500 .

Neptunea (Sipho) arata Verrill, Proc. U. S. Nat. Mus., 1880, vol. 3, p. 370 (non Gould). [south of Marthas Vineyard.]

ME.- Rockland; Sheepscot River.

MAss.- South of Marthas Vineyard, 64 to 192 fathoms.

*Colus stimpsonii brevis (Verrill).

Sipho stimpsonii var. brevis Verrill, Trans. Conn. Acad., 1882, vol. 5, p. 500. "off Chatham, [Mass.]."

Me.- Isle au Haute.

Mass.-Off Chatham, 17 fathoms; Ipswich.

- Colus pubescens (Verrill).

Sipho pubescens Verrill, Trans. Conn. Acad., 1882, vol. 5, p. 501, pl. 43, fig. 6. "off Cape Sable, N. S. ** off Martha's VineYARD." 
Neptunea propinqua Verrill, Amer. Journ. Sci., 1878, ser. 3, vol. 16, p. 210 (non Alder).

Gulf of Maine, off Cape Sable, 88 to 91 fathoms.

Mass.- Off Marthas Vineyard, 86 to 410 fathoms.

*Colus ventricosus (Gray).

Fusus ventricosus Gray, Zool. Beechey's Voy., 1839, p. 117. No locality. Gould, Inv. Mass., 1870, p. 373, fig. 640.

ME.

Mass.- Nantucket Shoals and Georges Bank (Stimpson).

*Colus pygmaeus (Gould).

Fusus islandicus var. pygmaeus Gould, Inv. Mass., 1841, p. 284, fig. 199. [New EngLand.]

Fusus trumbulli Linsley, Amer. Journ. Sci., 1845, ser. 1, vol. 48, p. 285, figs. 1, 2.

Neptunea (Neptunella) pygmaea Verrill, Rept. Inv. Vineyard Sound, 1873, p. 639, pl. 21, fig. 115.

Me.- Casco Bay, 10 to 20 fathoms; Frenchman's Bay; Eastport.

Mass.- Massachusetts Bay.

R. I.- Off Block Island, 29 fathoms; off Edgartown, 18 to 20 fathoms; off Gay Head, 19 fathoms.

Conn.- Off Stonington and New London.

\section{Colus pygmaeus planulus (Verrill).}

Sipho pygmaeus var. planulus Verrill, Trans. Conn. Acad., 1882, vol. 5, p. 505. " off Martha's Vineyard."

Mass.-Off Marthas Vineyard, 20 to 350 fathoms.

\section{Colus sabinii (Gray).}

Buccinum sabinii Gray, Suppl. to App. Parry's First Voyage, 1819-20, 1824, p. cexl. [Northwest Passage.] 
Sipho sabinii Verrill, Trans. Conn. Acad., 1882, vol. 5, p. 503, pl. 57 , fig. 23.

Dredged on Cashe's Ledge, Gulf of Maine.

*Busycon carica (Gmelin).

Gould, Inv. Mass., 1870, p. 383, fig. 646.

Murex carica Gmelin, Syst. Nat., 1790, vol. 1, pt. 6, p. 3545. No locality.

Fulgur carica Verrill, Rept. Inv. Vineyard Sound, 1873, p. 640, pl. 22, fig. 127 .

Mass.- Vineyard Sound, 1 to 10 fathoms; Nantucket.

R. I.- Narragansett Bay.

Conn.- Long Island Sound near New Haven.

*Busycon canaliculatum (Linné).

Gould, Inv. Mass., 1870, p. 380, fig. 645.

Murex canaliculatus Linné, Syst. Nat., ed. 12, 1767, vol. 1, p. 1222. "Ad Canadam."

Sycotypus canaliculatus Verrill, Rept. Inv. Vineyard Sound, 1873 , p. 640 .

Mass.- Vineyard Sound, 1 to 8 fathoms; Duxbury (introduced with seed oysters).

R. I.- Narragansett Bay.

Conn.- Long Island Sound near New Haven.

\section{PTYCHATRACTIDAE.}

*Ptychatractus ligatus (Mighels and Adams).

Stimpson, Amer. Journ. Conch., 1865, vol. 1, p. 59, pl. 8, fig. 8. Fasciolaria ligata Mighels and Adams, Boston Journ. Nat. Hist., 1842, vol. 4, p. 51, pl. 4, fig. 17. "Mingan in the Gulf of St. Lawrence."

Me.-Casco Bay, 20 to 40 fathoms; Eastport, 20 fathoms.

Conn.-Stonington, from codfish (Linsley). 


\section{MARGINELLIDAE.}

Marginella apicina borealis Verrill.

Dall, Bull. Mus. Comp. Zoöl., 1889, vol. 18, p. 136.

Marginella borealis Verrill, Trans. Conn. Acad., 1884, vol. 6, p. 165, pl. 29, fig. 4. “ OFF NORFolK, VA.”

Mass.- Off Marthas Vineyard, 64.5 to 100 fathoms.

\section{CANCELLARIIDAE.}

\section{*Admete couthouyi (Jay).}

Cancellaria buccinoides Couthouy, Boston Journ. Nat. Hist., 1838, vol. 2, p. 105, pl. 3, fig. 3 (non Sowerby, 1832). “MAss[ACHUSETTS] BAY."

Cancellaria couthouyi Jay, Cat. Shells, 1839, ed. 3, p. 77.

Admete viridula Stimpson, Shells of New England, 1851, p. 44. Gould, Inv. Mass., 1870, p. 391, fig. 652 (non Fabricius).

Me.-Casco Bay; Frenchman's Bay, 25 to 30 fathoms; Eastport, 10 fathoms.

Mass.- Massachusetts Bay; Duxbury, 20 fathoms; off Nauset Light.

\section{TURRITIDAE.}

Drillia dalli (Verrill and Smith).

Dall, Bull. U. S. Nat. Mus., 1889, no. 37, p. 98, pl. 60, figs. 66, 66 a.

Pleurotoma dalli Verrill and Smith in Verrill, Trans. Conn. Acad., 1882, vol. 5, p. 451, pl. 57, figs. 1, 1a. “ off Martha's Vineyard, *** off Delaware Bay."

Mass.-Off Marthas Vineyard, 94 to 146 fathoms.

Drillia carpenteri (Verrill and Smith).

Pleurotoma carpenteri Verrill and Smith in Verrill, Amer. Journ. 
Sci., 1880, ser. 3, vol. 20, pp. 391, 395. [off Marthas Vineyard.] Verrill, Trans. Conn. Acad., 1882, vol. 5, p. 452, pl. 57, fig. 2.

MAss.- Off Marthas Vineyard, 86 to 155 fathoms.

\section{*Bela nobilis (Möller).}

G. O. Sars, Moll. Reg. Aret. Norv., 1878, p. 228, pl. 16, figs. 19, 20.

Defrancia nobilis Möller, Krøyer's Naturh. Tidskr., 1842, vol. 4, p. 85. [GREENLAND.]

ME.-Frenchman's Bay, 10 to 15 fathoms; Eastport.

\section{Bela scalaris (Möller).}

Verrill, Trans. Conn. Acad., 1882, vol. 5, p. 471, pl. 57, figs. 12, $12 \mathrm{a}$.

Defrancia scalaris Möller, Krroyer's Naturh. Tidskr., 1842, vol. 4, p. 85. " Groenland."

Bela turricula Gould, Inv. Mass., 1870, p. 351, fig. 620.

Bela americana Packard, Mem. Boston Soc. Nat. Hist., 1867, vol. 1, p. 285 , pl. 7 , fig. 11.

Me.- Broad Sound, Casco Bay and off Halfway Light, 14 to 29 fathoms; Eastport, 10 fathoms.

N. H.- Isles of Shoals, 25 fathoms; Jeffrey's Ledge, 15 fathoms.

Mass.- Massachusetts Bay, 29 to 40 fathoms; off Cape Cod, 15 to 32 fathoms; Duxbury, 20 to 30 fathoms.

\section{Bela rugulata gouldii Verrill.}

Bela gouldii Verrill, Trans. Conn. Acad., 1882, vol. 5, p. 462, pl. 57, figs. 6, 6a. "Massachusetts Bay *** Gulf of Maine."

ME.-Casco Bay, 17 to 30 fathoms; Frenchman's Bay, 25 to 30 fathoms.

Mass.- Massachusetts and Cape Cod Bays, 15 to 50 fathoms; off Cape Cod, 122 fathoms. 
*Bela cancellata (Mighels and Adams).

Verrill, Trans. Conn. Acad., 1882, vol. 5, p. 475, pl. 43, figs. 10, 11; pl. 57, fig. 13 .

Fusus cancellatus Mighels and Adams, Boston Journ. Nat. Hist., 1842, vol. 4, p. 52, pl. 4, fig. 18. "CAsco BAy."

ME.- Frenchman's Bay, south of Egg Rock Light, 25 fathoms; Eastport, 10 fathoms.

Mass.- Massachusetts Bay and off Cape Cod, 12 to 92 fathoms; off Marthas Vineyard, 126 to 312 fathoms.

*Bela decussata (Couthouy).

Gould, Inv. Mass., 1870, p. 354, fig. 623. Verrill, Trans. Conn. Acad., 1882, vol. 5, p. 479, pl. 43, fig. 13.

Pleurotoma decussata Couthouy, Boston Journ. Nat. Hist., 1839, vol. 2, p. 183, pl. 4, fig. 8. " Massachusetts Bay."

Bela decussata var. tenuicostata Verrill, Trans. Conn. Acad., 1882, vol. 5, p. 481 (non Sars).

ME.-Casco Bay; Frenchman's Bay, 10 to 12 fathoms; Eastport.

Mass.-Off Cape Ann, 38 to 75 fathoms; off Cape Cod, 28 to 30 fathoms.

Bela decussata pusilla Verrill.

Verrill, Trans. Conn. Acad., 1882, vol. 5, p. 481. "Casco Bay." ME.-Casco Bay, 12 to 15 fathoms; Frenchman's Bay; Wiscasset.

\section{*Bela exarata (Möller).}

G. O. Sars, Moll. Reg. Arct. Norv., 1878, p. 232, pl. 16, fig. 18.

Defrancia exarata Möller, Krфyer's Naturh. Tidskr., 1842, vol. 4, p. 85. [GreEnLAND.]

Tritonium mitrula Lovén, Öfvers. Kongl. Vet.-Akad. Förh., Stockholm, 1846, vol. 3, p. 145. 
Bela concinnula Verrill, Trans. Conn. Acad., 1882, vol. 5, p. 468, pl. 43 , fig. 15 .

Me.-Casco Bay; Frenchman's Bay, 10 to 12 fathoms; Gulf of Maine, 25 to 118 fathoms.

Mass.- Massachusetts Bay, 16 to 29 fathoms; off Cape Cod, 25 to 122 fathoms.

\section{*Bela rosea Sars.}

Verrill, Trans. Conn. Acad., 1882, vol. 5, p. 485.

Bela harpularia var. rosea G. O. Sars, Moll. Reg. Aret. Norv., 1878, p. 234, pl. 23, fig. 10. “Trons $\varnothing$ og Lofoten *** VestKrst (Christiansund) [Norway]."

Me.-Penobscot Bay.

Mass.-Duxbury, 20 fathoms; Georges Bank.

*Bela incisula Verrill.

Verrill, Trans. Conn. Acad., 1882, vol. 5, p. 461, pl. 43, fig. 12; pl. 57, fig. 14. " off Newport, R. I., NORTHWARd to Labrado:.."

Bela impressa? Verrill, Proc. U. S. Nat. Mus., 1880, vol. 3, p. 365 (non Mörch).

Gulf of Maine, 10 to 70 fathoms.

Me.-Casco Bay, 10 fathoms; Frenchman's Bay, 10 to 35 fathoms; Eastport.

Mass.- - Salem Harbor, 5 fathoms; Massachusetts and Cape Cod Bays, 13 to 30 fathoms; off Cape Cod, 26 to 67 fathoms; off Marthas Vineyard, 28 fathoms.

R. I.-Off Newport, 27 fathoms.

Bela blaneyi Bush.

Bush, Nautilus, 1909, vol. 23, p. 61, fig. 1. "south of EGG ROCK BUOY iN ABOUT 30 FATHOMS, ${ }^{* * *}$ FrenchMAN'S Bay, Maine."

ML.-Frenchman's Bay. 
*Bela harpularia (Couthouy).

Gould, Inv. Mass., 1870, p. 352, fig. 621. Verrill, Trans. Conn. Acad., 1882, vol. 5, p. 473, pl. 43, fig. 14; pl. 57, fig. 9.

Fusus harpularius Couthouy, Boston Journ. Nat. Hist., 1838, vol. 2, p. 106, pl. 1, fig. 10. "Mass[Achusetts] Bay."

Me.-Casco Bay; Frenchman's Bay, 15 to 50 fathoms; Eastport, 10 to 50 fathoms.

Mass.- Massachusetts Bay, 8 to 29 fathoms; off Cape Cod, 15 to 34 fathoms; off Gay Head, 18 to 29 fathoms.

R. I.-Off Block Island, 20 to 28 fathoms.

*Bela pleurotomaria (Couthouy).

Gould, Inv. Mass., 1870, p. 355, fig. 625. Verrill, Trans. Conn. Acad., 1882, vol. 5, p. 478.

Fusus pleurotomarius Couthouy, Boston Journ. Nat. Hist., 1838, vol. 2, p. 107, pl. 1, fig. 9. "Mass[AChUsetts] Bay."

Me.-Casco Bay; Frenchman's Bay, 10 to 50 fathoms; Eastport, 10 fathoms.

Mass.- Massachusetts Bay, 31 to 48 fathoms; off Cape Cod, 30 to 122 fathoms; off Chatham, 16 fathoms.

*Bela bicarinata (Couthouy).

Pleurotoma bicarinata Couthouy, Boston Journ. Nat. Hist., 1838, vol. 2, p. 104, pl. 1, fig. 11. "Mass[AChUsetts] BaY." Gould, Inv. Mass., 1870, p. 349, fig. 618.

Me.-Casco Bay; Frenchman's Bay, 35 fathoms; Eastport, 10 to 50 fathoms.

Mass.-Off Cape Ann, 38 fathoms; off Cape Cod, 27 to 31 fathoms; Vineyard Sound, 6 to 12 fathoms.

Conv.- Stonington.

*Bela bicarinata violacea (Mighels and Adams).

Pleurotoma violacea Mighels and Adams, Proc. Boston Soc. Nat. 
Hist., 1841(?), vol. 1, p. 50. "CASco BAY." Boston Journ. Nat. Hist., 1842, vol. 4, p. 51, pl. 4, fig. 21.

ME.- Frenchman's Bay, 10 to 35 fathoms; Eastport, 10 to 50 fathoms.

Mass.- Massachusetts Bay; Georges Bank, 45 to 60 fathoms.

Bela pingelii (Möller).

Verrill, Trans. Conn. Acad., 1882, vol. 5, p. 464, pl. 43, fig. 16.

Defrancia pingelii Möller, Krọver's Naturh. Tidskr., 18+2, vol. 4, p. 86. [GReENLAND.]

Me.- Eastport, 20 to 90 fathoms.

Mass.- Off Cape Cod, 34 fathoms; Georges Bank.

Bela tanneri (Verrill and Smith).

Dall, Bull. U. S. Nat. Mus., 1889, no. 37, p. 100, pl. 61, fig. 78.

Typhlomangelia tanneri Verrill and Smith in Verrill, Trans. Conn. Acad., 1884, vol. 6, p. 163, pl. 31, fig. 8. “N. LATITUde $40^{\circ} 16^{\prime} 50^{\prime \prime}$, W. LONGITUde $67^{\circ} 05^{\prime} 15^{\prime \prime}$ IN 1290 FATHOMS."

Gulf of Maine and Georges Bank (Dall).

*Mangilia plicosa (C. B. Adams).

Dall, Bull. U. S. Nat. Mus., 1889, no. 37, p. 100, pl. 50, fig. 14.

Plcurotoma plicata C. B. Adams, Boston Journ. Nat. Hist., 1S40, vol. 3, p. 318, pl. 3, fig. 6 (non Lamarck). "Harbour of NeW BEDFord [MLAss.]."

Plcurotoma plicosa C. B. Adams, Contr. to Conch., 1850, no. 4, p. 54 .

Mass.-Dartmouth; New Bedford Harbor; North Falmouth. Conn.- Near New Haven.

Mangilia cerina (Kurtz and Stimpson).

Verrill, Trans. Conn. Acad., 1882, vol. 5, p. 488, fig. 1.

Pleurotoma cerinum Kurtz and Stimpson, Proc. Boston Soc. 
Nat. Hist., 1851, vol. 4, p. 115. “Buzzard's BAY AND*** South Carolina."

Mass.- Vineyard Sound and Buzzards Bay, 3 to 12 fathoms; New Bedford.

R. I.- Near Newport; near Watch Hill.

Conn.- Near New Haven.

\section{Mangilia comatotropis (Dall).}

Dall, Bull. Mus. Comp. Zoöl., 1889, vol. 18, p. 116, pl. 11, fig. 12. Pleurotoma (Mangilia) comatotropis Dall, Bull. Mus. Comp. Zoöl., 1881, vol. 9, p. 58. "CAPE San Antonio, 640 FMs."

Mass.- Off Marthas Vineyard, 100 to 487 fathoms.

Pleurotomella packardii Verrill.

Verrill, Amer. Journ. Sci., 1873, ser. 3, vol. 5, p. 15. [N. LAT. $42^{\circ} 5^{\prime}$, W. LONG. $67^{\circ} 45^{\prime}, 110$ fathoms.] Verrill, Trans. Conn. Acad., 1882 , vol. 5, p. 453 , pl. 43 , fig. 9 ; pl. 57, fig. 5 .

Gulf of Maine, 85 to 110 fathoms.

Mass.- Off Cape Cod, 96 fathoms.

\section{Pleurotomella agassizii (Verrill and Smith).}

Verrill, Trans. Conn. Acad., 1882, vol. 5, p. 454, pl. 57, figs. 3, 3a. Pleurotoma agassizii Verrill and Smith in Verrill, Amer. Journ. Sci., 1880, ser. 3, vol. 20, p. 394. [off Marthas Vineyard.]

Mass.- South of Marthas Vineyard, 65 to 500 fathoms.

\section{Pleurotomella (Gymnobela) blakeana (Dall).}

Dall, Bull. Mus. Comp. Zoöl., 1889, vol. 18, p. 126, pl. 10, fig. 1.

Pleurotoma (Bela) blakeana var. normalis Dall, Bull. Mus. Comp. Zoöl., 1881, vol. 9, p. 54. “off CAPE San Antonio, 640 FMS."

Gymnobela brevis Verrill, Trans. Conn. Acad., 1885, vol. 6, p. 417.

Mass.-Off Nantucket Shoals; Georges Bank; Gulf of Maine. 
ACTEONIDAE.

Acteon punctostriatus (C. B. Adams).

Tornatella puncto-striata C. B. Adams, Boston Journ. Nat. Hist., 1840, vol. 3, p. 323, pl. 3, fig. 9. "New Bedford [Mass.]." Actaeon punctostriatus Pilsbry, Manual Conch., 1893, ser. 1, vol. 15, p. 157, pl. 18, figs. 98, 99; pl. 19, figs. 22, 23.

MLass.- Vineyard Sound and Buzzards Bay; South Dennis.

R. I.- Narragansett Bay.

Cons.- Stonington, from haddock (Linsley); near New Haven.

TORNATINIDAE.

*Tornatina canaliculata (Say).

Pilsbry, Manual Conch., 1893, ser. 1, vol. 15, p. 184, pl. 22, fig. 23 ; pl. 50, figs. 25, 26.

Volvaria canaliculata Say, Journ. Acad. Nat. Sci. Phila., 1826, ser. 1, vol. 5, p. 211. "South CarolinNa."

Utriculus canaliculatus Gould, Inv. Mass., 1870, p. 219, fig. 510.

Me.- Portland Harbor (Fuller).

Mass.-Massachusetts Bay; Vineyard Sound and Buzzards Bay, 2 to 8 fathoms.

R. I.- Narragansett Bay; near Watch Hill.

Conn.-Stonington; near New Haven.

\section{*Retusa pertenuis (Mighels).}

Pilsbry, Manual Conch., 1893, ser. 1, vol. 15, p. 216, pl. 23, figs. $48,49$.

Bulla pertenuis Mighels, Boston Journ. Nat. Hist., 1843, vol. 4, p. 346, pl. 16, fig. 3. "CAsco BaY."

Utriculus pertenuis Gould, Inv. Mass., 1870, p. 218, fig. 509.

Me.-Casco Bay; Frenchman's Bay, 4 to 10 fathoms.

Mass.- Massachusetts Bay; Duxbury. 
Retusa obtusa turrita (Möller).

Pilsbry, Manual Conch., 1893, ser. 1, vol. 15, p. 215, pl. 23, fig. 52. Bulla turrita Möller, Krórer's Naturh. Tidskr., 1842, vol. 4, p. 79. [GREENLAND.]

Bulla incincta Mighels, Proc. Boston Soc. Nat. Hist., 1844, vol. 1, p. 188.

Me.-Casco Bay (Mighels); Frenchman’s Bay (Blaney).

\section{Retusa gouldii (Couthouy).}

Pilsbry, Manual Conch., 1893, ser. 1, vol. 15, p. 217, pl. 23, figs. 58, 59 .

Bulla gouldii Couthouy, Boston Journ. Nat. Hist., 1839, vol. 2, p. 181, pl. 4, fig. 6. "Massachusetts Bay."

Utriculus gouldii Gould, Inv. Mass., 1870, p. 217, fig. 508.

Me.-Casco Bay; Frenchman's Bay, 4 to 10 fathoms.

Mass.-Stellwagens Bank, 15 to 25 fathoms; from fish taken off Cohasset; Provincetown; Georges Bank.

\section{Retusa mayoi (Dall).}

Utriculus mayoi Dall, Bull. Mus. Comp. Zoöl., 1889, vol. 18, p. 46. "Portland, Maine."

ME.-- Portland, from fish stomach (Mayo).

Retusa obesiuscula (Brugnone).

Pilsbry, Manual Conch., 1893, ser. 1, vol. 15, p. 231, pl. 23, figs. 60,61 .

Cylichna obesiuscula Brugnone, Bull. Soc. Mal. Ital., 1877, vol. 3, p. 39, pl. 1, fig. 7. "Ficarazzi [Pliocene of Palermo]."

Diaphana conulus Verrill, Trans. Conn. Acad., 1882, vol. 5, p. 543, pl. 58, fig. 25 (non Deshayes).

MAss.-Off Marthas Vineyard, 100 to 155 fathoms. 


\section{SCAPHANDRIDAE.}

Scaphander punctostriatus (Mighels and Adams).

Gould, Inv. Mass., 1870, p. 215, fig. 505.

Bulla puncto-striata Mighels and Adams, Proc. Boston Soc. Nat. Hist., 1841 (?), vol. 1, p. 49 . "CAsco BAy." Mighels and Adams, Boston Journ. Nat. Hist., 1842, vol. 4, p. 43, pl. 4, fig. 10.

Me.- Casco Bay; Gulf of Maine.

Mass.- Massachusetts Bay.

\section{Diaphana debilis (Gould).}

Gould, Inv. Mass., 1870, p. 216, fig. 507.

Bulla debilis Gould, Amer. Journ. Sci., 1St0, ser. 1, vol. 38, p. 196.

ME.-Casco Bay, 6 fathoms; Frenchman's Bay, 10 to 16 fathoms; Eastport.

Mass.-- Massachusetts Bay; Duxbury.

Conn.- Stonington, from stomach of cod (Linsley).

Diaphana hiemalis (Couthouy).

Gould, Inv. Mass., 1870, p. 216, fig. 506.

Bulla hiemalis Couthouy, Boston Journ. Nat. Hist., 1839, vol. 2, p. 180, pl. 4, fig. 5. " off Provincetown [, Mass.]."

Me.- Casco Bay; Bristol.

Mass.- Provincetown, from fish.

Diaphana globosa (Lovén).

Pilsbry, Manual Conch., 1S93, ser. 1, vol. 15, p. 2S6, pl. 26, fig. 75.

Amphisphyra globosa Lovén, Öfvers. Kongl. Tet.-Akad. Förh., 1846, vol. 3, p. 143. "BoH[uslan]-Finm[ARk, Norway]." Verrill, Trans. Conn. Acad., 1882, vol. 5, p. 543.

Mass.- Off Marthas Vineyard, 115 to 361 fathoms (Terrill). 
*Cylichna alba (Brown).

Gould, Inv. Mass., 1870, p. 220, fig. 511.

Volvaria alba Brown, Illustr. Conch. Great Brit., 1827, pl. 38, figs. 43, 44. "Greinock [, England]."

Bulla triticea Couthouy, Boston Journ. Nat. Hist., 1838, vol. 2, p. 88 , pl. 2 , fig. 8 .

Me.-Casco Bay; Frenchman's Bay, 10 to 30 fathoms; Eastport.

Mass.-- Massachusetts Bay, off Nahant, 17 fathoms; Duxbury; Georges Bank.

R. I.- Narragansett Bay; near Block Island.

Conn.- Stonington, from haddock stomach (Linsley).

Cylichna occulta (Mighels and Adams).

Pilsbry, Manual Conch., 1893, ser. 1, vol. 15, p. 292, pl. 28, figs. $35-41$.

Bulla occulta Mighels and Adams, Journ. Boston Soc. Nat. Hist., 1842, vol. 4, p. 54, pl. 4, fig. 11. [Westbrook, MaIne (fossil).]

ME.

Cylichna oryza (Totten).

Gould, Inv. Mass., 1870, p. 221, fig. 512.

Bulla oryza Totten, Amer. Journ. Sci., 1835, ser. 1, vol. 28, p. 350, fig. 5. "Newport harbor, (R. I.)."

ME.

Mass.-Vineyard Sound and Buzzards Bay; New Bedford Harbor, 4 fathoms.

R. I.- Newport.

Conn.- Near New Haven; Stratford.

BULLARIIDAE.

Bullaria gemma (Verrill).

Diaphana (Utriculus) gemma Verrill, Amer. Journ. Sci., 1880, ser. 3, vol. 20, pp. 392, 399. [south of Marthas Vineyard.] 
Verrill, Trans. Conn. Acad., 1882, vol. 5, p. 543, pl. 58, fig. 22.

Bulla gemma Pilsbry, Manual Conch., 1893, ser. 1, vol. 15, p. 339.

Mass.-South of Marthas Vineyard, 100 to 115 fathoms.

\section{*Haminea solitaria (Say).}

Pilsbry, Manual Conch., 1893, ser. 1, vol. 15, p. 357, pl. 28, fig. $44 ;$ pl. 41, fig. 32 .

Bulla solitaria Say, Journ. Acad. Nat. Sci. Phila., 1822, ser. 1, vol. 2, p. 245. " southern coast of the United States."

MAss.-Massachusetts Bay; shores of Vineyard Sound and Buzzards Bay; Duxbury.

R. I.- Newport; Mark Rock; White Camp; near Watch Hill. Conn.- New Haven Harbor, near Fort Hale.

\section{RINGICULIDAE.}

Ringicula nitida Verrill.

Verrill, Amer. Journ. Sci., 1873, ser. 3, vol. 5, p. 16. [NORTH oF the Georges Bank, N. LAt. $42^{\circ} 5^{\prime}$, W. Long. $67^{\circ} 49^{\prime}, 110$ And 150 fathons.] Verrill, Trans. Conn. Acad., 1874, vol. 3, p. 48, pl. 1, fig. 2 ; Ibid., 1882, vol. 5, p. 540.

MAss.- Off Marthas Vineyard, 100 to 300 fathoms.

\section{PHILINIDAE.}

\section{Philine sinuata Stimpson.}

Stimpson, Proc. Boston Soc. Nat. Hist., 1850, vol. 3, p. 333. "Boston Harbor, [Mass.]." Gould, Inv. Mass., 1870, p. 213, fig. 502 .

Me.- Casco Bay.

Mass.-- Broad Bay, Boston Harbor, 4 to 7 fathoms (Stimpson); Duxbury. 


\section{Philine quadrata (S. V. Wood).}

Gould, Inv. Mass., 1870, p. 213, fig. 503.

Bulla quadrata S. V. Wood, Charlesworth's Mag. Nat. Hist., 1839, vol. 3, p. 461, pl. 7, fig. 1. "CORALline CraG, Sutton, [ENGLAND]."

Philine formosa Stimpson, Proc. Boston Soc. Nat. Hist., 1850, vol. 3, p. 334 .

ME.

Mass.-Off Cape Ann, 30 fathoms; Massachusetts Bay; Duxbury, 20 fathoms; Georges Bank.

*Philine lima (Brown).

Pilsbry, Manual Conch., 1895, ser. 1, vol. 16, p. 20, pl. 5, figs. 711.

Utriculus lima Brown, Illustr. Recent Conch. Great Brit., 1844, ed. 2, p. 58, pl. 19, figs. 39, 40. "Greenock [England]."

Bulla lineolata Couthouy, Boston Journ. Nat. Hist., 1839, vol. 2, p. 179 , pl. 3, fig. 15 .

Philine lineolata Gould, Inv. Mass., 1870, p. 214, fig. 504.

Me.- Frenchman's Bay, off Egg Rock.

Mass.- Massachusetts Bay, from stomachs of fishes; Georges Bank.

Philine amabilis Verrill.

Verrill, Amer. Journ. Sci., 1880, ser. 3, vol. 20, pp. 392, 398. [south of Marthas Vineyard.] Verrill, Trans. Conn. Acad, 1882, vol. 5, p. 544, pl. 58, figs. 23, 24.

Mass.- South of Marthas Vineyard, 120 and 130 fathoms.

\section{Philine cingulata G. O. Sars.}

G. O. Sars, Moll. Reg. Arct. Norv., 1878, p. 297, pl. 26, figs. 7a7c. [Lofoten, Norway, 120 to 200 fathoms.] Verrill, Trans. Conn. Acad., 1882, vol. 5, p. 544.

Gulf of Maine, off Cape Sable, 90 fathoms. 
Philine fragilis G. O. Sars.

G. O. Sars, Moll. Reg. Arct. Norv., 1878, p. 296, pl. 18, figs. 11a-11c. "Vads $\phi$, [Norway]." Verrill, Trans. Conn. Acad., 1882, vol. 5, p. 544.

Gulf of Maine, Jefirey's Ledge, 88 to 92 fathoms; off Cape Sable, 90 fathoms.

\section{Philine finmarchica M. Sars.}

M. Sars in G. O. Sars, Moll. Reg. Arct. Norv., 1878, p. 296, p. 18, figs. 10a-d. [Finmark.] Verrill, Trans. Conn. Acad., 1882, vol. 5 , p. 544 .

Gulf of Maine, off Cape Sable, 90 fathoms.

Off Cape Cod, 16 fathoms; off Marthas Vineyard, 86 fathoms.

Philine tincta Verrill.

Verrill, Trans. Conn. Acad., 1882, vol. 5, p. 544. [off Marthas VINEYARD.]

Mass. - South of Marthas Vineyard, 65 fathoms.

\section{Philine angulatá Jeffreys.}

Jeffreys, Brit. Conch., 1867, vol. 4, p. 451. "LARne, co. Antrim, Hebrides, and Shetland." Pilsbry, Manual Conch., 1895, ser. 1, vol. 16, p. 17, pl. 3, figs. 41, 42 .

Me.-Casco Bay (Kingsley).

\section{APLYSIIDAE}

Tethys willcoxi (Heilprin).

Pilsbry, Manual Conch., 1895, ser. 1, vol. 16, p. 80, pl. 35, figs. 30-32.

Aplysia willcoxi Heilprin, Proc. Acad. Nat. Sci. Phila., 1886, p. 364; Trans. Wagner Free Inst. Sci., 1887, vol. 1, p. 130, pl. 19. "Little Gasparilla Bay, west coast of Florida." 
Mass.- Katama Bay, Oct. 31, 1900; Buzzards Bay, Oct. 11, 1906; New Bedford; Westport, Lamberts Cove; 'Tarpaulin Cove; Robinsons Hole and Menemsha Bight (Sumner, Osburn, and Cole).

\section{PLEUROBRANCHAEIDAE.}

Pleurobranchaea tarda Verrill.

Verrill, Amer. Journ. Sci., 1880, ser. 3, vol. 20, pp. 392, 398. [south of Marthas Vineyard.] Verrill, Trans. Conn. Acad., 1882, vol. 5, p. 546, pl. 58, fig. 26.

Mass.- South of Marthas Vineyard, 28 to 250 fathoms, abundant in 45 and 69 fathoms.

R. I.- South of Block Island, 38 fathoms.

Koonsia obesa Verrill.

Verrill, Trans. Conn. Acad., 1882, vol. 5, p. 545. “off Martha's Vineyard." Verrill, Rept. U. S. Comm. Fish and Fisheries for 1883, 1885, p. 571, pl. 28, fig. 107. Pilsbry, Manual Conch., 1895, ser. 1, vol. 16, p. 222, pl. 74, fig. 94.

Mass.- Marthas Vineyard, 192 to 258 fathoms.

PTEROPODA.

Superfamily Thecosomata.

LIMACINIDAE.

Limacina retroversa (Fleming).

Pelseneer, Rept. Voy. Challenger, Zool., 1888, vol. 23, art. 1, p. 27.

Fusus retroversus Fleming, Mem. Wern. Nat. Hist. Soc., 1823, vol. 4, p. 498, pl. 15, fig. 2. "Noss Island, Zetland."

Heterofusus retroversus Gould, Inv. Mass., 1870, p. 505, pl. 27, figs. $345-348$.

Heterofusus balea Gould, Inv. Mass., 1870, p. 505, pl. 27, fig. 349. 
Spirialis gouldii Stimpson, Proc. Boston Soc. Nat. Hist., 1851, vol. 4 , p. 8 .

Me.-Casco Bay.

Mass.- Abundant at Nahant during the summer of 1863 (A. Agassiz); Massachusetts Bay, Feb. to Apr. (Stimpson); near Naushon Island. This species was found alive at about 100 different localities in the Gulf of Maine by the "Grampus" in July and August, 1913 and 1914.

Limacina retroversa macandrei (Forbes and Hanley).

Spirialis macandrei Forbes and Hanley, Brit. Moll., 1853, vol. 2, p. 385, pl. 57, figs. 6, 7. "FIFTEEN MiLes to the south of Mizen Head, south of Ireland."

Spirialis retroversus var. macandrei Verrill, Trans. Conn. Acad., 1884, vol. 6, p. 275 .

MASS.- South of Marthas Vineyard.

Limacina helicina (Phipps).

Pelseneer, Rept. Voy. Challenger, Zool., 1888, vol. 23, art. 1, p. 21.

Clio helicina Phipps, A Voyage towards the North Pole, 1774, p. 195. "ARCTICK SEAS."

Gulf of Maine.

Limacina inflata (d'Orbigny).

Atlanta inflata d'Orbigny, Voy. Amér. Mérid., 1836, vol. 5, p. 174, pl. 12, figs. 16-19. "Océan atlantique ET DANS LE GRAND OCÉAN."

Spirialis rostralis Verrill, Trans. Conn. Acad., 1885, vol. 6, p. 431.

"Grampus" Exp. 1914, N. lat. $39^{\circ} 54^{\prime}$, W. long. $70^{\circ} 43^{\prime}$, alive; and N. lat. $42^{\circ} 11^{\prime}$, W. long. $69^{\circ} 59^{\prime}$, alive. 


\section{CAVOLINIDAE.}

\section{Cavolina telemus (Linné).}

Dall, Bull. Mus. Comp. Zoöl., 1908, vol. 43, p. 230.

Monoculus telemus Linné, Syst. Nat., ed. 10, 1758, vol. 1, p. 635.

"Algiriae."

Anomia tridentata Forskil, Descr. Anim. Orient. Observ., 1775, p. 124.

Cawolina tridentata Verrill, Trans. Conn. Acad., 1882, vol. 5, p. 554, figs. 6,7 .

Mass.- Off Marthas Vineyard, 19 and 22 fathoms; "Grampus " Exp. 1914 , N. lat. $40^{\circ} 39^{\prime}$, W. long. $67^{\circ} 28^{\prime}$, alive.

R. I.- Newport, from stomach of a bluefish, off Block Island.

\section{Cavolina uncinata (Rang).}

Verrill, Trans. Conn. Acad., 1882, vol. 5, p. 554.

Hyalaca uncinata Rang (MIS.) in d'Orbigny, Vò̀. Amér. Mérid., 1836, vol. 5, p. 93, pl. 5, figs. 11-15. "Atlantique, $24^{\circ}$ S. LAt."

Mass.- Off Marthas Vineyard, 65 to 487 fathoms; "Grampus" Exp. 1914 , N. lat. $40^{\circ} 6^{\prime}$, W. long. $68^{\circ} 6^{\prime}$, alive.

\section{Cavolina longirostris (Lesueur).}

Verrill, Trans. Conn. Acad., 1882, vol. 5, p. 555.

IIyalaca longirostris Lesueur (MS.) in Blainville, Dict. des Sci. Nat., 1821, vol. 22, p. 81 . "OCÉAN Atlantique, $22^{\circ} 9^{\prime}$ [N. LAT.]."

Mass.- Off Marthas Vineyard, 64 to 500 fathoms; " Grampus " Exp. 1914 , N. lat. $40^{\circ} 6^{\prime}$, W. long. $68^{\circ} 6^{\prime}$, alive.

\section{Cavolina gibbosa (Rang).}

Verrill, Trans. Conn. Acad., 1884, vol. 6, p. 213.

Hyalaea gibbosa Rang (MS.) in d'Orbigny, Voy. Amér. Mérid., 1836, vol. 5, p. 95, pl. 5, figs. 16-20. [South AtLantic.]

\section{MAss.- South of Marthas Vineyard.}


Cavolina trispinosa (Lesueur).

Hyalaea trispinosa Lesueur (MS.) in Blainville, Dict. des Sci. Nat., 1821, vol. 22, p. 82. " LA Mer DES Antilles."

Diacria trispinosa Gould, Inv. Mass., 1870, p. 504.

Mass.-Off Marthas Vineyard; occasionally cast ashore on Nantucket; "Grampus" Exp. 1913, 10 miles N N W. of Eastern Point, Gloucester, alive; “Grampus” Exp. 1914, N. lat. $40^{\circ} 6^{\prime}$, W. long. $68^{\circ} 6^{\prime}$, alive.

Diacria quadridentata (Lesueur).

Tesch, F. E. Schulze's Das Tierreich, 1913, pt. 36, Pteropoda, p. 41 .

Hyalaea quadridentata Lesueur (MS.) in Blainville, Dict. des Sci. Nat., 1821, vol. 22, p. 81. "OcÉan Atlantique, *** LAT. $23^{\circ}$ $36^{\prime}$, LONGIT. $27^{\circ} 22^{\prime}$."

Off Georges Bank, N. lat. $40^{\circ} 16^{\prime} 50^{\prime \prime}$, W. long. $67^{\circ} 05^{\prime} 15^{\prime \prime}$ (Verrill).

Creseis acicula Rang.

Rang, Ann. des Sci. Nat., 1828, vol. 13, p. 318, pl. 17, fig. 6. "L'OcÉAn et LA MER DEs INDES."

Creseis recta Dall, Bull. U. S. Nat. Mus., 1889, no. 37, p. 80, pl. 66, fig. 118.

Near Georges Bank, N. lat. $41^{\circ} 25^{\prime}$, W. long. $65^{\circ} 30^{\prime}$, Sept. 15, 1872, at surface (Verrill); “Grampus" Exp. 1914, N. lat. 40 6', W. long. $68^{\circ} 6^{\prime}$.

Creseis virgula Rang.

Rang, Ann. des Sci. Nat., 1828, vol. 13, p. 316, pl. 17, fig. 2. " l'Océan atlantique et les Antilles."

Styliola virgula Verrill, Trans. Conn. Acad., 1882, vol. 5, p. 557.

Near Georges Bank, N. lat. $41^{\circ} 25^{\prime}$, W. long. $65^{\circ} 30^{\prime}$, Sept. 15, 1872, at surface (Verrill). 
Creseis conica Eschscholtz.

Eschscholtz, Zool. Atlas, 1829, pt. 3, p. 17, pl. 15, fig. 3. "KüsTE. von Brastlien."

Styliola vitrea Verrill, Amer. Journ. Sci., 1872, ser. 3, vol. 3, p. 284, pl. 6, fig. 7 .

Mass.- Taken at the surface among Salpae, off Gay Head, Sept. 9, 1871 (Verrill); “Grampus" Exp. 1914, N. lat. 40 6', W. long. $68^{\circ} 6^{\prime}$.

Styliola subula (Quoy and Gaimard).

Cleodora subula Quoy and Gaimard, Ann. des Sci. Nat., 1827, vol. 10, p. 233, pl. 8d, figs. 1-3, " LA CÔTE De TénérifFe."

Styliola subulata Verrill, Trans. Conn. Acad., 1884, vol. 6, pl. 213.

Mass.- South of Marthas Vineyard, N. lat. $39^{\circ} 49^{\prime}$, W. long. $68^{\circ} 28^{\prime} 30^{\prime \prime}$ (Verrill).

\section{Hyalocyclis striata (Rang).}

Creseis striata Rang, Ann. des Sci. Nat., 1828, vol. 13, p. 315, pl. 17, fig. 3. "Océan atlantique et la mer des Indes."

Clio (Hyalocylix) striata Pelseneer, Rept. Voy. Challenger, Zool., 1888, vol. 23, art. 1, p. 54, pl. 2, fig. 3 .

“Grampus" Exp. 1914, N. lat. 406', W. long. $68^{\circ} 6^{\prime}$, alive.

Clio pyramidata Linné.

Linné, Syst. Nat., ed. 12, 1767, vol. 1, p. 1094. " IN Oceano." Verrill, Trans. Conn. Acad., 1882, vol. 5, p. 555.

Mass.-- South of Marthas Vineyard, N. lat. $40^{\circ} 05^{\prime}$, W. long. $70^{\circ} 23^{\prime}, 65$ to 500 fathoms (Verrill).

\section{Clio recurva (Children).}

Balantium recurvum Children, Journ. Roy. Inst., 1829, vol. 15, p. 220 , pl. 7 , fig. 107 . 
Clio balantium Pelseneer, Rept. Voy. Challenger, Zool., 1888, vol. 23, art. 1, p. 61.

Mass.-Off Marthas Vineyard, N. lat. $40^{\circ} 05^{\prime}$, W. long. $70^{\circ} 23^{\prime}$ (Verrill).

\section{Cuvierina columnella (Rang).}

Pelseneer, Rept. Voy. Challenger, Zool. 1888, vol. 23, art. 1, p. 67.

Cuvieria columnella Rang, Ann. des Sci. Nat., 1827, vol. 12, p. 323, pl. 45, B, figs. 1-8. "Océan atlantique."

Triptera columnella Verrill, Trans. Conn. Acad., 1882, vol. 5, p. $557 ; 1884$, vol. 6 , p. 214.

Mass. - South of Marthas Vineyard, N. lat. $39^{\circ} 55^{\prime} 28^{\prime \prime}$, W. long. $69^{\circ} 47^{\prime}$ (Verrill); "Grampus" Exp. 1914, N. lat. $40^{\circ} 6^{\prime}$, W. long. $68^{\circ} 6^{\prime}$.

\section{PERACLIDIDAE.}

Peracle reticulata (d'Orbigny).

Dall, Bull. U. S. Nat. Mus., 1889, no. 37, p. 80.

Atlanta reticulata d'Orbigny, Voy. Amér. Mérid., 1836, vol. 5, p. 178 , pl. 12 , figs. $32-34$. [S. LAT. $20^{\circ}$, W. LONG. $89^{\circ}$.]

"Grampus" Exp. 1914, N. lat. $40^{\circ} 6^{\prime}$, W. long. $68^{\circ}$ 6', alive.

\section{CYMBULIIDAE.}

Corolla calceola (Verrill).

Dall, Bull. U. S. Nat. Mus., 1889, no. 37, p. 200, explanation of plate 66 , fig. 120 .

Cymbulia calceola Verrill, Amer. Journ. Sci., 1880, ser. 3, vol. 20, pp. 392, 394. [NEAR N. LAT. $40^{\circ} 05^{\prime}$, W. LONG. $70^{\circ} 23^{\prime}$.] Verrill, Trans. Conn. Acad., 1882, vol. 5, p. 553, pl. 58, fig. 33.

Mass.-South of Marthas Vineyard; "Grampus" Exp. 1914, N. lat. $40^{\circ} 6^{\prime}$, W. long. $68^{\circ} 6^{\prime}$; one mile off Gay Head, Aug. 27, 1890 (Edwards).

R. I.- ESE. of Block Island at surface, Oct. 2, 1880. 
Superfamily Grmnosomata.

CLIONIDAE.

Clione limacina (Phipps).

Gould, Inv. Mass., 1870, p. 507, fig. 754.

Clio limacina Phipps, A Voyage towards the North Pole, 1774, p. 195. [ARCTIC SEAS.]

Clione papillonacea Verrill, Rept. Inv. Vineyard Sound, 1873, p. 668.

ME.-Portland, April and May, 1868.

Mass.- - Vineyard Sound; Woods Hole.

Conn.-Off Stonington.

"Grampus" Exp. 1913, 1914, at many stations in the Gulf of Maine.

Paedoclione doliiformis Danforth.

Danforth, Proc. Boston Soc. Nat. Hist., 1907, vol. 34, p. 2, pls. 1-4. "CASco BaY."

Me.-Casco Bay, plankton, nights of Aug. 28 and Sept. 5-8, 1902.

NUDIBRANCHIA.

HERMAEIDAE.

Hermaea cruciata Gould.

Gould (A. Agassiz Ms'.), Inv. Mass., 1870, p. 253, pl. 17, fig. 256. "Naushon Island [, Mass.]."

Mass.- Naushon Island, Sept., 1863 (A. Agassiz).

Stiliger fuscatus (Gould).

Calliopaea (?) fuscata Gould, Inv. Mass., 1870, p. 250, pl. 16, figs. 218-221. "Boston."

Stiliger fuscata Rathbun, Proc. U. S. Nat. Mus., 1880, vol. 3, p. 127.

MAss.-Boston, on logs in a mast yard, July 22, 1842 (Gould). 
Alderia harvardiensis Gould.

Gould, Inv. Mass., 1870, p. 254, pl. 16, figs. 226-228. "CAMBRIDGE, [MASS.]."

Mass.- "Found in great numbers, in brackish waters, at Cambridge, April, 1848" (Agassiz); Cohasset in marsh creeks.

\section{EIXSIIDAE.}

\section{Elysia chlorotica Gould.}

Gould (A. Agassiz MS.), Inv. Mass., 1870, p. 255, pl. 17, figs. 251-255. "Cambridge [, Mass.]."

Mass.- Found in great numbers in brackish water on the Cambridge marshes, in the spring of 1848 (Agassiz); Cohasset on eel-grass; Duxbury.

Elysia (Elysiella) catula (Gould).

?Placobranchus simplex Girard, Proc. Boston Soc. Nat. Hist., 1854, vol. 5, p. 89.

Placobranchus catulus Gould (A. Agassiz MS.), Inv. Mass., 1870, p. 256, pl. 17, figs. 249, 250. "EAst Boston."

Elysiella catulus Verrill, Amer. Journ. Sci., 1872, ser. 3, vol. 3, p. 284, pl. 7, figs. 5, 5a; Rept. Inv. Vineyard Sound, 1873, pp. 480, 668, pl. 25, fig. 171.

Elysia catulus Apgar, Journ. Trenton (N. J.) Nat. Hist. Soc., 1891, vol. 2, p. 129, pl. 3, fig. 26.

MAss.- " Channel near East Boston, January, 1848" (Agassiz); Chelsea Beach (Girard); Woods Hole, adhering to eel-grass; Duxbury.

Conn.-- New Haven.

\section{LIMAPONTIIDAE}

Limapontia zonata (Girard).

Gould, Inv. Mass., 1870, p. 258.

Niobe zonata Girard, Proc. Boston Soc. Nat. Hist., 1852, vol. 4, p. 211. "Boston Harbor, [Mass.]." 
AEOLIDIIDAE.

*Aeolidia papillosa (Linné).

Limax papillosus Linné, Fauna Suecica, 1761, ed. 2, p. 508. "In Mari Norvegico."

Eolis farinacea Stimpson, Smithson. Contr. Knowl., 1854, vol. 6, art. 5, p. 25.

Acolis papillosa Gould, Inv. Mass., 1870, p. 238, fig. 518, pl. 18, figs. $257,261$.

ME.-Casco Bay; Eastport.

MAss.- Boston; Rocky Neck and Brace's Cove, East Gloucester, Apr. 1908 (Balch).

R. I.- Watch Hill, among roots of Laminariae (Verrill).

\section{CRATENIDAE.}

Cuthona aurantia (Alder and Hancock).

Eolis aurantia Alder and Hancock, Ann. Mag. Nat. Hist., 1842, ser. 1, vol. 9, p. 34. "Whitley [, England]."

Eolis aurantiaca Alder and Hancock, Monogr. Brit. Nud. Moll., 1851, pt. 5, fam. 3, pl. 27; 1855, pt. 7, pl. 47, fig. 21.

Montagua gouldii Verrill, Rept. Inv. Vineyard Sound, 1873, p. 667.

Cuthona aurantiaca Sars, Moll. Reg. Arct. Norv., 1878, p. 321, pl. 28, figs. 6a, 6b; pl. 16, fig. 7 .

Cratena gymnota Verrill and Emerton in Verrill, Proc. U. S. Nat. Mus., 1880, vol. 3, p. 390 (in part).

Mass.- Massachusetts Bay.

Conn.-- New Haven; off Thimble Island, Long Island Sound.

Cratena gymnota (Couthouy).

Bergh, Syst. der Nud. Gaster., 1892, p. 31.

Eolis (Tergipes, Cuv.) gymnota Couthouy, Boston Journ. Nat. Hist., 1838, vol. 2, p. 69, pl. 1, fig. 3. " tide water of Charles River, Mass." 
Aeolis gymnota Gould, Inv. Mass., 1870, p. 249, fig. 520 (ova), pl. 16, figs. 238-241.

Mass.- Charles River, near Craigie's Bridge, Boston; Warren Bridge, about the roots of Tubularia, Oct. and Nov., 1847 (Couthouy); Woods Hole (Verrill); Cohasset, Nov. (Owen Bryant).

\section{Cratena pilata (Gould).}

Bergh, Syst. der Nud. Gaster., 1892, p. 31.

Aeolis pilata Gould, Inv. Mass., 1870, p. 243, pl. 19, figs. 270, 277, 279, 281. "Charles River, [Mass.]."

Acolis vermiferus Smith, Ann. Lyc. Nat. Hist. N. Y., 1870, vol. 9, p. 391.

Arolidia pilatu and Hontagna pilata Verrill, Rept. Inv. Vineyard Sound, 1873, pp. 383, 666, pl. 25, fig. 174.

Mass. - Charles River, in Sept., on timbers (Gould); Duxbury. Conn.- New Haven Harbor, on piles of Long Wharf (Verrill); Thimble Island, Long Island Sound (Verrill).

Cratena veronicae Verrill.

Verrill, Proc. U. S. Nat. Mus., 1880, vol. 3, p. 389. " "off CAPE Con."

Mass.- Off Cape Cod, in 23 to 31 fathoms, among hydroids, Sept. 6, 1879; Cohasset, Sept. 1906, on Laminariae (Owen Bryant); Rocky Neck, Gloucester Harbor, Apr. 1908 (Balch).

Cratena? pustulata (Alder and Hancock).

Bergh, Malacol. Unters., 1878, vol. 15, p. 18.

Eolis pustulata Alder and Hancock, Monogr. Brit. Nud. Moll., 1855, pt. 7, fam. 3, pl. 45, figs. 4, 5; pl. 47, fig. 22. "CullerCOATs, [EnGLAND]."

Eolis purpurea Stimpson, Smithson. Contr. Knowl., 1854, vol. 6, art. 5, p. 25.

Aeolis purpurea Gould, Inv. Mass., 1870, p. 246. 
Grand Manan, "Duck Island, under stones at low-water" (Stimpson).

\section{Tergipes despectus (Johnston).}

Verrill, Rept. Inv. Vineyard Sound, 1873, pp. 495, 667; Proc. U. S. Nat. Mus., 1880, vol. 3, p. 391.

Eolidia despecta Johnston, Loudon's Mag. Nat. Hist., 1835, vol. 8, p. 378, fig. 35e. " Berwick Bay [England]."

Aeolis despecta Gould, Inv. Mass., 1870, p. 248, pl. 16, figs. 222225 (in part according to Verrill, see Galvina exigua).

Me.-- Casco Bay; Eastport Harbor.

Mass.- Timber dock, Charles River, Sept., 1842; South Boston, July; on piles of Warren Bridge, low tide, among Campanularia, June and July (Stimpson teste Gould); Gloucester (Gould).

R. I.- Off Watch Hill, 4 to 5 fathoms, on Laminaria, among hydroids (Verrill).

Conn.- Stonington.

Embletonia fuscata Gould.

Gould, Inv. Mass., 1870, p. 251, pl. 16, figs. 229-232. “CHARLes River *** South Boston Bridge, [Mass.]."

Mass.-Charles River, on logs in timber dock; South Boston Bridge, on Laomedea geniculata, July, 1842 and 1865 (Gould); Provincetown among the filamentous green algae (Rathbun); Duxbury.

Embletonia fuscata lanceolata Gould.

Gould, Inv. Mass., 1870, p. 252, fig. 521. No locality.

Mass.

Embletonia remigata Gould.

Gould, Inv. Mass., 1870, p. 252, pl. 16, figs. 214-217. "Charles River and at South Boston Bridge, [Mass.]."

MAss.-Charles River and at South Boston Bridge, with $E$. fuscata, in July, upon Laomedea geniculata. 
Galvina picta (Alder and Hancock).

Sars, Moll. Reg. Arct. Norv., 1878, p. 461, tab. anat. struct. pl. 16, fig. 5 (dent.).

Eolis pallida Alder and Hancock, Ann. Mag. Nat. Hist., 1842, ser. 1, vol. 9, p. 35. "CUllercoats SANDS [England]."

Eolis picta Alder and Hancock, Monogr. Brit. Nud. Moll., 1847, pt. 3, fam. 3, pl. 33 .

Aeolis picta Gould, Inv. Mass., 1870, p. 246, pl. 19, fig. 282.

Mass.-Timber dock, Boston, 1842, (Gould); dredged in 5 fathoms, near Govemor's Id., Boston Harbor, June, 1850 (Stimpson teste Gould); Rocky Neck, Gloucester Harbor, 1908 (Balch).

Galvina exigua (Alder and Hancock).

Sars, Moll. Reg. Arct. Norv., 1878, p. 461, tab. anat. struct. pl. 16, fig. 6 (dent.).

Eolis exigua Alder and Hancock, Ann. Mag. Nat. Hist., 1S4S, ser. 2, vol. 1, p. 192. "Fowey Harbour [England]." Alder and Hancock, Monogr. Brit. Nud. Moll., 1851, pt. 5, fam. 3, pl. 37.

Aeolis despecta Gould, Inv. Mass., 1870, p. 248, pl. 16, figs. 222225 (in part, see Verrill, Trans. Conn. Acad., 1882, vol. 5, p. 553).

Mass. - Salem, 1879, on Obelia (Emerton); Boston, spring and early summer.

\section{CORYPHELLIDAE.}

Coryphella rufibranchialis (Johnston).

Sars, Moll. Reg. Aret. Norv., 1878, p. 319.

Eolis rufibranchialis Johnston, Loudon's Mag. Nat. Hist., 1S32, vol. 5, p. 428, fig. 85. " Berwick BAy, [EnGLAND]." Alder and Hancock, Monogr. Brit. Nud. Moll., 1848, pt. 4, fam. 3, pl. 14; 1855, pt. 7, pl. 47, fig. 9 (dent.).

Aeolis rufibranchialis Gould, Inv. Mass., 1870, p. 242, pl. 19, figs. 269, 272 (in part according to Verrill, see $C$. $r$. mananensis).

Mass. 
Coryphella rufibranchialis mananensis (Stimpson).

Balch, Nautilus, 1909, vol. 23, p. 35.

Eolis mananensis Stimpson, Smithson. Contr. Knowl., 1854, vol. 6, art. 5, p. 26 . "IN 35 F[ATHoms], ** Hake Bay [GRand Manan]."

Aeolis rufibranchialis Gould, Inv. Mass., 1870, p. 242 (in part according to Verrill).

Me.-Casco Bay; Kennebunk; Eastport.

Dredged in many localities from off Fisher's and Block Island to Halifax, N. S., in 20 to 90 fathoms, among hydroids, the most common species at such depths (Verrill).

Mass.-Rocky Neck, Gloucester Harbor, April 26-30, 1908 (Baleh); Cohasset, Nov. (Bryant).

Coryphella rufibranchialis chocolata Balch.

Balch, Nautilus, 1909, vol. 23, p. 35. "Rocky Neck [Mass.]."

Mass.-Rocky Neck, Gloucester Harbor, April 26-30, 1908; Bass Rocks, May 20, 1915 (Balch).

Coryphella stellata (Stimpson).

Bergh, Syst. der Nud. Gaster., 1892, p. 36.

Eolis stellata Stimpson, Smithson. Contr. Knowl., 1854, vol. 6, art. 5, p. 25. [Grand Manan.]

Aeolis stellata Gould, Inv. Mass., 1870, p. 245, pl. 19, figs. 271, 278 .

Grand Manan.

Coryphella(?) salmonacea (Couthouy).

Eolis (Cavolina, Brug.) salmonacea Couthouy, Boston Journ. Nat. Hist., 1838, vol. 2, p. 68, pl. 1, fig. 2. "Charles River, Mass." 
Aeolis salmonacea Gould, Inv. Mass., 1870, p. 240, pl. 18, figs. $264,265$.

Mass._- "Tide-water of Charles River," Boston.

\section{Coryphella diversa (Couthouy).}

Bush, Proc. U. S. Nat. Mus., 1883, vol. 6, p. 242.

Eolis diversa Couthouy, Boston Journ. Nat. Hist., 1839, vol. 2, p. 187, pl. 4, fig. 14 (not fig. 9). “Massachusetts Bay, Chelsea BeAch."

Aeolis diversa Gould, Inv. Mass., 1870, p. 247, pl. 19, figs. 267, 268 (not fig. 280, ?fig. 276).

Mass.- Massachusetts Bay, spring, among roots of Laminaria saccharina.

Coryphella stimpsoni (Verrill).

Verrill, Proc. U. S. Nat. Mus., 1880, vol. 3, p. 388; 'Trans. Conn. Acad., 1882, vol. 5, p. 552, pl. 42, fig. 14.

Cuthona stimpsoni Verrill, Amer. Journ. Sci., 1879, ser. 3, vol. 17, p. 314. "Еastport, Me."

Me.- Eastport, low water; Jeffreys Ledge, 51 fathoms.

Mass.-Salem Harbor; Massachusetts Bay.

Coryphella rutila Verrill.

Verrill, Amer. Journ. Sci., 1879, ser. 3, vol. 17, p. 314. “EAsTPORT, ME." Verrill, Trans. Conn. Acad., 1882, vol. 5, p. 552.

ME.- Eastport at low water.

Coryphella nobilis Verrill.

Verrill, Proc. U. S. Nat. Mus., 1880, vol. 3, p. 388. " off CAPE Con." Verrill, Trans. Conn. Acad., 1882, vol. 5, p. 552, pl. 42, fig. 15 .

Off Cape Cod, in 75 fathoms, Sept. 1, 1879 (Verrill). 


\section{FACELINIDAE.}

Facelina bostoniensis (Couthouy).

Verrill, Proc. U. S. Nat. Mus., 1880, vol. 3, p. 389.

Eolis bostoniensis Couthouy, Boston Journ. Nat. Hist., 1838, vol. 2, p. 67, pl. 1, fig. 1. " "Tide water of Charles River, Mass."

Aeolis bostoniensis Gould, Inv. Mass., 1870, p. 241, pl. 19, figs. 266, 273-275, 283.

Mass.-Sept. with ova at Craigie's Bridge, Charles River; rather common in various parts of Boston Harbor by dredging, and at Lynn (Stimpson teste Gould); Cohasset, Sept., on Laminaria (Owen Bryant); common from low water to 20 fathoms, on Obelia and other hydroids, from Massachusetts Bay to Block Id. and Newport, R. I. (Verrill).

\section{FIONIDAE.}

Fiona marina (Forskål).

Bergh, Rept. Voy. Challenger, Zool., 1884, vol. 10, art. 1, p. 9, pl. 11, fig. 1 .

Limax marinus Forskål, Deser. Anim. Orient., Observ. 1775, p. 99; Icon. Rer. Nat. in Itinere Orient., 1776, pl. 26, fig. G. g. "Mari Mediterraneo."

Fiona nobilis Alder and Hancock, Monogr. Brit. Nud. Moll., 1855, pt. 7, fam. 3, pl. 38a; pl. 47, fig. 30 (dent.). Verrill, 'Trans. Conn. Acad., 1882, vol. 5, p. 551.

South of Marthas Vineyard, in large numbers among Anatifera, on pieces of floating timber (Verrill).

\section{GLAUCIDAE.}

\section{Glaucus atlanticus Forster.}

Forster, Voy. "Resolution," 1777, vol. 1, p. 49. [South AtranTIC.] Bergh, Rept. Voy. Challenger, Zool., 1884, vol. 10, art. 1, p. 16, pl. 14, fig. 16 .

Glaucus margaritaceus Verrill, Trans. Conn. Acad., 1885, vol. 6, 
p. 430; Rept. U. S. Comm. Fish and Fisheries for 1883, 1885, p. 592, pl. 28, figs. $113,113 \mathrm{a}, 113 \mathrm{~b}$.

South of Marthas Vineyard, pelagic; N. lat. $39^{\circ} 05^{\prime} 30^{\prime \prime}, \mathrm{W}$. long. $70^{\circ} 44^{\prime} 30^{\prime \prime}$.

\section{DOTOIDAE.}

Doto coronata (Gmelin).

Gould, Inv. Mass., 1870, p. 236, fig. 517 (ova), pl. 16, figs. 233237 .

Doris coronata Gmelin, Syst. Nat., 1790, p. 3105. "AD LITTUS insula Walcheren SeElandiae."

Me.- Casco Bay; Bay of Fundy.

Mass. - Charles River on piles of Craigie's Bridge, below lowwater mark; Nahant; off Marthas Vineyard; Rocky Neck, Gloucester Harbor.

R. I.- Off Watch Hill, 4 to 5 fathoms on Obelia.

Conn.-- Long Island Sound, near New Haven.

Doto formosa Verrill.

Verrill, Amer. Journ. Sci., 1875, ser. 3, vol. 10, p. 41, pl. 3, fig. 4. " off Point Judith [R. I.] 10-14 Fathoms."

ME.- Eastport, from low-water mark to 50 fathoms, usually on hydroids (Verrill).

Mass.- Off Point Judith, 10 to 14 fathoms, Aug. 19.

\section{DENDRONOTIDAE.}

Dendronotus frondosus (Ascanius).

Amphitrite frondosa P. A. Ascanius, Kongl. Vid. Selsk. Skrift., 1774, vol. 5, p. 158, pl. 5, fig. 2. "IN MARr Norvegico."

Tritonia arborescens Gould, Inv. Mass., 1841, p. 5.

Dendronotus arborescens Gould, Inv. Mass., 1870, p. 234, pl. 22, figs. $311-313$.

Tritonia reynoldsii Couthouy, Boston Journ. Nat. Hist., 1838, vol. 2, p. 74, pl. 2, figs. 1-4. 
Me.-Casco Bay; Kennebunk; also at Grand Manan.

Mass.-Craigie's Bridge, Boston (Gould); Cohasset, Nov.; Rocky Neck and Brace's Cove, Gloucester (Balch); Duxbury. Common among hydroids.

R. I.- Watch Hill, 4 to 5 fathoms, common on Laminaria among Obelia (Verrill).

\section{Dendronotus frondosus elegans Verrill.}

Dendronotus elegans Verrill, Proc. U. S. Nat. Mus., 1880, vol. 3, p. 385. "off Cape CoD." Verrill, Trans. Conn. Acad., 1882, vol. 5, p. 551.

Dendronotus arborescens Bergh, Syst. der Nud. Gaster., 1892, p. 59 .

Off Cape Cod, 26 fathoms, Sept. 6, 1879.

\section{Dendronotus robustus Verrill.}

Verrill, Amer. Journ. Sci., 1870, ser. 2, vol. 50, p. 405, fig. 1, a-c. “Whale Cove, Grand Menan." Verrill, Proc. U. S. Nat. Mus., 1879, vol. 2, p. 197; Trans. Conn. Acad., 1882, vol. 5, p. 550.

Dendronotus velifer Sars, Moll. Reg. Aret. Norv., 1878, p. 315, pl. 28, fig. 2; tab. anat. struct. pl. 15, fig. 4 (dent.).

Me.-Casco Bay; Eastport.

Mass.- Ipswich Bay, 19 fathoms, July; Cohasset on kelp with D. frondosus, November (Owen Bryant); south of Marthas Vineyard, in 192 fathoms (Verrill).

\section{SCYLLAEIDAE.}

* Scyllaea pelagica marginata Bergh.

Bergh, Verh. Zool.-Bot. Ges. Wien, 1871, vol. 21, p. 1292.

Scyllaea edwardsii Verrill, Amer. Journ. Sci., 1878, ser. 3, vol. 16, p. 211; Trans. Conn. Acad., 1882, vol. 5, p. 550, pl. 42, fig. 10.

Mass.-Woods Hole, in Little Harbor, attached to eel-grass and sargassum, autumn of 1877 (Verrill); Vineyard Sound on sargassum (Gray). 
HOLOHEPATICA.

DORIDIDAE.

Geitodoris complanata (Verrill).

Bergh, Bull. Mus. Comp. Zoöl., 1894, vol. 25, p. 163, pl. 4, figs. 13-18; pl. 5, figs. 1-5.

Doris complanata Verrill, Amer. Journ. Sci., 1880, ser. 3, vol. 20, pp. 392, 399. [South of Marthas Vineyard, 86 Fathoms.] Verrill, Trans. Conn. Acad., 1882, vol. 5, p. 549, pl. 58, figs. 34-34b.

Mass.- South of Marthas Vineyard, 85 to 146 fathoms, among sponges (Verrill).

Cadlina obvelata (Müller).

Doris obvelata Müller, Zoöl. Danicae Prodr., 1776, p. 229. [ DENMARK.]

Doris planulata Gould, Inv. Mass., 1870, p. 231, pl. 20, figs, 294, 296.

Doris repanda Verrill, Amer. Journ. Sci., 1879, ser. 3, vol. 17, p. 312 .

Cadlina repanda Bergh, Bull. Mus. Comp. Zoöl., 1894, vol. 25, p. 169, pl. 7, figs. 4-11.

Me.-Casco Bay, 3 to 30 fathoms; Gulf of Maine, 60 to 150 fathoms (Verrill); Passamaquoddy Bay, July (Gould); Frenchman's Bay (Dwight Blaney); Blue Hill Bay, on stones, 10 fathoms, Sept.

MAss.- Cohasset, on stones, 5 fathoms, August.

\section{POLYCERIDAE.}

Issa lacera (Abildgaard).

Bergh, Verh. Zool.-Bot. Ges. Wien, 1881, vol. 30, p. 646, pl. 13, figs. 12-15; pl. 14, figs. 4-12. Verrill, Trans. Conn. Acad., 1882, vol. 5 , p. 547 , pl. 42 , fig. 11 .

Doris lacera Abildgaard in Müller, Zool. Danicae, 1806, vol. 4, p. 23, pl. 138, figs. 3-4. " IN MARI Norvegico." 
Cashe's Ledge.

Off Cape Ann, 25 to 80 fathoms; Massachusetts Bay, 35 to 48 fathoms; off Cape Cod, 70 fathoms; on and near Stellwagens Bank, 25 to 60 fathoms, August (Mayer and Balch).

Issa ramosa Verrill and Emerton.

Verrill and Emerton in Verrill, Amer. Journ. Sci., 1881, ser. 3, vol. 22, p. 301. [south of Marthas Vineyard.] Verrill, Trans. Conn. Acad., 1882, vol. 5, p. 547, pl. 58, figs. 36, 36a.

South of Marthas Vineyard, 100 to 130 fathoms.

Palio lessonii (d'Orbigny).

Bergh, Verh. Zool.-Bot. Ges. Wien, 1881, vol. 29, p. 602.

Polycera lessonii d'Orbigny, Mag. de Zool., 1837, vol. 7, art. 8, cl. 5, p. 5, pl. 105. "Environs DE LA Rochelle [France]." Gould, Inv. Mass., 1870, p. 226, pl. 17, figs. 242-248.

Doris illuminata Gould, Inv. Mass., 1841, p. 4.

ME.-Common in Casco Bay and Bay of Fundy, low water to 20 fathoms (Verrill).

Mass.-Craigie's Bridge and Aspinwall's shipyard (Gould); Cohasset, on kelp, Nov. (Owen Bryant).

R. I.- Watch Hill, 3 to 6 fathoms.

Conn.- Savin Rock, near New Haven, and off south end, 4 to 5 fathoms (Verrill).

\section{Polycerella emertoni Verrill.}

Verrill, Proc. U. S. Nat. Mus., 1880, vol. 3, p. 387. "Wood's Holl [, Mass.]*** New Haven, Conn.*** Newport, R. I." Verrill, Trans. Conn. Acad., 1882, vol. 5, p. 548. Bergh, Verh. Zool.-Bot. Ges. Wien, 1884, vol. 33, p. 162, pl. 8, figs. 9-19; pl. 9, figs. 1-6 (anat.).

Mass. - Woods Hole, among eel-grass, Aug. and Sept.

R. I.- Newport, on filamentous algae, July and August (Verrill).

Conv. - New Haven, on hydroids from piles of Long Wharf, Oct. (Verrill). 
GONIODORIDAE.

Acanthodoris pilosa (Abildgaard).

Doris pilosa Abildgaard, in Müller, Zool. Danica, 1789, vol. 3, p. 7, pl. 85, figs. 5-8. " "IN FUCIS MARIS NorvagicI."

Doris bifida Verrill, Amer. Journ. Sci., 1870, ser. 2, vol. 50, p. 406. Acanthodoris stellata Verrill, Amer. Journ. Sci., 1879, ser. 3, vol. 17, p. 313 .

Acanthodoris citrina Verrill, Amer. Journ. Sci., 1879, ser. 3, vol. 17 , p. 313.

ME.- Eastport, at low-water mark, Aug. 19, 1868.

Mass.- Charlestown Navy Yard, 1849 (Gould); Brace's Cove, East Gloucester, Apr. 1908 (Balch).

Conn.- Savin Rock, near New Haven, low-water mark, egglaying, Oct.

\section{Acanthodoris pilosa ornata (Verrill).}

Acanthodoris ornata Verrill, Amer. Journ. Sci, 1879, ser. 3, vol. 17, p. 313. “Eastport, ME.” Verrill, Trans. Conn. Acad., 1882, vol. 5, p. 549, pl. 42, fig. 12. Bergh, Syst. der Nud. Gaster., 1892, p. 159 .

ME.- Eastport, at low-water mark, August (Verrill).

Adalaria proxima (Alder and Hancock).

Bergh, Dall's Sci. Results Expl. Alaska, 1880, art. 6, p. 225, pl. 9, figs. 12-15.

Doris proxima Alder and Hancock, Monogr. Brit. Nud. Moll., 1854, pt. 6, fam. 1, pl. 9, figs. 10-16; 1855, pt. 7, pl. 46, fig. S (dent.). "Birkenhead, [ENGJand]."

ME.- Eastport, at low-water mark (Verrill).

\section{Lamellidoris muricata (Müller).}

Bergh, Dall's Sci. Results Expl. Alaska, 1850, art. 6, p. 221, pl. 9, fig. 18; pl. 11, figs. 10-12. 
Doris muricata Müller, Zool. Danicae Prodr., 1776, p. 229. [DENMARK.]

Onchidoris muricata Verrill, Amer. Journ. Sci., 1879, ser. 3, vol. 17, p. 314.

Me.- Eastport.

R. I.--Block Island.

*Lamellidoris aspera (Alder and Hancock).

Bergh, Syst. der Nud. Gaster., 1892, p. 161.

Doris aspera Alder and Hancock, Ann. Mag. Nat. Hist., 1842, ser. 1, vol. 9, p. 32. "Trnemouth, Cullercoats and Whitley, [EnGLAND]."

Doris pallida Gould, Inv. Mass., 1870, p. 229, pl. 20, figs. 284, 287, 288 (not fig. 291).

Onchidoris pallida Verrill, Amer. Journ. Sci., 1870, ser. 2, vol. 50, p. 408 .

Me.-Casco Bay; Eastport Harbor, low water to 30 fathoms (Verrill).

Mass.- Beverly, Feb., 1848 (Agassiz); dredged near Governor's Id., Boston Harbor, Oct., 1849 (Stimpson teste Gould); Cohasset, on kelp, Nov. (Owen Bryant); off Cuttyhunk Id. (Verrill); Brace's Cove, East Gloucester, on corallines, Apr. 1908 (Balch).

R. I.- Newport.

Lamellidoris diaphana (Alder and Hancock).

Verrill, Trans. Conn. Acad., 1882, vol. 5, p. 550.

Doris diaphana Alder and Hancock, Ann. Mag. Nat. Hist., 1845, ser. 1, vol. 16, p. 313 . "Meadfoot Sands, Torbay, [England]." Alder and Hancock, Monogr. Brit. Nud. Moll., 1846, pt. 2, fam. 1, pl. 10 ; 1855 , pt. 7 , pl. 46 , fig. 9 (dent.).

ME.-Eastport, at low-water mark (Verrill). 
*Lamellidoris bilamellata (Linné).

Bergh, Semper's Reisen Archipel d. Philippinen, pt. 2, 1878, vol. 2 , no. 14, p. 606 , pl. 64 , fig. 13 ; pl. 65 , figs. $1-5$.

Limax bilamellatus Linné, Fauna Suecica, 1761, ed. 2, p. 508. "Maris Norvegici."

Doris bilamellata Gould, Inv. Mass., 1870, p. 228, pl. 20, figs. 285, 286; pl. 21, figs. 299, 305-309.

Mass.- Under a floating log at East Boston, Maý, 1849 (Gould); Beverly, June (Agassiz); Boston Harbor near Governor's Id., 4 fathoms, May, 1853 (Stimpson); Castle Id., Boston Harbor, May, 1915 (Humphrey).

Lamellidoris (?) tenella (Gould).

Bergh, Syst. der Nud. Gaster., 1892, p. 161.

Doris tenella Gould, Inv. Mass., 1870, p. 229, pl. 20, figs. 289, 290, 293. " BeverLy, [MAss.]."

Onchidoris tenclla Verrill, Amer. Journ. Sci., 1870, ser. 2, vol. 50, p. 407.

ME.- Near Eastport, low-water mark, 1870 (Verrill).

Mass.- Beverly, February, 1848 (Agassiz).

\section{Lamellidoris (?) grisea (Gould).}

Bergh, Syst. der Nud. Gaster., 1892, p. 161.

Doris grisea (Stimpson MS.) Gould, Inv. Mass., 1870, p. 232, pl. 20, figs. 292, 295. "Charles Rrver *** Chelsea BEACH *** East Boston Point, [Mass.]."

Onchidoris grisea Verrill, Amer. Journ. Sci., 1870, ser. 2, vol. 50, p. 407 .

ME.- Near Eastport, in a pool at low-water mark (Verrill).

Mass.-- On floating Zostera in Charles River, November 9, 1842; also on the under side of Fucus-covered stone at East Boston Point, in April (Stimpson teste Gould). 
Lamellidoris (?) diademata Gould.

Bergh, Syst. der Nud. Gaster., 1892, p. 161.

Doris diademata Gould, Inv. Mass., 1870, p. 230, pl. 21, figs. 298, 300-304. "Boston and Beverly Harbors [, Mass.]."

Mass.- " Found in deep water, Boston and Beverly Harbors" (Agassiz).

Idalia (Idaliella) pulchella Alder and Hancock.

Idalia pulchella Alder and Hancock, Ann. Mag. Nat. Hist., 1854, ser. 2, vol. 14, p. 103. " "ST. Ives, [England]." Alder and Hancock, Monogr. Brit. Nud. Moll., 1854, pt. 6, fam. 1, pl. 17, figs. 5, 6; 1855, pt. 7, App., p. v.

Idaliella pulchella Verrill, Trans. Conn. Acad., 1882, vol. 5, p. 548 , pl. 42 , fig. 13 .

Mass.-Salem.

Idalia (Idaliella) modesta Verrill.

Idalia modesta Verrill, Amer. Journ. Sci., 1875, ser. 3, vol. 10, p. 41, pl. 3, fig. 3. [off Fisher's Island; Block Island Sound; N. of Little Gull Island, 40 fathoms.]

Idaliella modesta Verrill, Trans. Conn. Acad., 1882, vol. 5, p. 548.

Mass.- Vineyard Sound and off No Man's Land.

R. I.-Block Island Sound, 17 to 24 fathoms, Aug. 6.

Conn.- Off west end of Fisher's Island, Aug. 25, 1874 (Verrill).

\section{Ancula cristata sulphurea Stimpson.}

Ancula sulphurea Stimpson, Smithson. Contr. Knowl., 1854, vol. 6, art. 5, p. 26. [Grand Manan.] Gould, Inv. Mass., 1870, p. 233, fig. 516 (ova), pl. 22 , figs. 310,314 .

Mass.-Among corallines, on Zostera, and under stones in Boston Harbor; Brace's Cove, East Gloucester, Apr. 1908 (Balch). 


\section{CORAMBIDAE。}

Doridella obscura Verrill.

Verrill, Amer. Journ. Sci., 1870, ser. 2, vol. 50, p. 408, figs. 2, a-b, 3. "Savin Rock near New Haven [, Conn.]." Verrill, Rept. Inv. Vineyard Sound, 1873, pp. 664, 400, pl. 25, fig. 173, a-b; Trans. Conn. Acad., 1882, vol. 5, p. 547, fig. 5 .

Mass.- Vineyard Sound, low water to 10 fathoms.

R. I.- Off Block Island, 13 fathoms.

Conn.- Savin Rock, Oct. 28, 1868; Long Island Sound, near New Haven, low water to 5 fathoms.

PULMONATA.

BASOMMATOPHORA.

\section{AURICULIDAE.}

*Carychium exiguum (Say).

Gould, Inv. Mass., 1870, p. 466, fig. 720. Pilsbry, Proc. Acad. Nat. Sci. Phila., 1891, p. 319.

Pupa exigua Say, Journ. Acad. Nat. Sci. Phila., 1822, ser. 1, vol. 2, p. 375. [near Philadelphia, Pa.]

Me.- Kennebunkport; Orono; Woodland, Aroostook Co.; Bethel; Mt. Desert.

Mass.-Boston; Lexington; Westfield.

R. I.- Providence.

Conn.- New Haven.

${ }^{*}$ Carychium exile H. C. Lea.

H. C. Lea, Amer. Journ. Sci., 1842, ser. 1, vol. 42, p. 109, pl. 1, fig. 5. "Wissahiccon creek, near Philadelphia." Pilsbry, Nautilus, 1894, vol. 8, p. 63, figs. 10-14.

Me.-Caribou; Woodland; Hebron; Buckfield.

\section{Carychium exile canadense Clapp.}

Clapp, Nautilus, 1906, vol. 19, p. 139, pl. 8, figs. 1, 2, 6, 7.

"Kennebunkport, Me." 
Me.-Kennebunkport; Islesboro; Eagle Lake; Caribou, Aroostook Co.

VT.- Hartland.

Carychium minimum Müller.

Müller, Verm. Terr. et Fluv. Hist., 1774, vol. 2, p. 125. [EuROPE.] Forbes and Hanley, Brit. Moll., 1853, vol. 4, p. 198, pl. 125, fig. 6 .

Mass.- Norfolk Downs, in the vicinity of greenhouses. Introduced.

*Alexia myosotis (Draparnaud).

Gould, Inv. Mass., 1870, p. 463, figs. 718, 719.

Auricula myosotis Draparnaud, Tabl. Moll. France, 1801, p. 53, pl. 3, figs. 16, 17. " Côtes de la Méditerranée."

Auricula denticulata Gould, Inv. Mass., 1841, p. 199, fig. 129 (non Montfort).

Me.-Portland; Eastport; Castine.

Mass.-Boston; Woods Hole; Marblehead.

R. I.- Newport; Wickford.

Conn.-Stonington; New Haven; West River, near West Haven; Branford.

${ }^{*}$ Melampus lineatus Say.

Say, Journ. Acad. Nat. Sci. Phila., 1822, ser. 1, vol. 2, p. 246. "Maryland and New Jersey."

Melampus bidentatus Say, Journ. Acad. Nat. Sci. Phila., 1822, ser. 1, vol. 2, p. 245 (non Montagu, 1803). Gould, Inv. Mass., 1870, p. 467, fig. 721.

Me. - Portland; Biddeford Pool; New Meadows River.

Mass.--Ipswich; Revere; Plymouth; Provincetown; Woods Hole.

R. I.- Nayatt; Narragansett Bay.

Conn.- New Haven. 
PHYSIDAE.

*Physa heterostropha (Say).

Gould, Inv. Mass., 1870, p. 483, fig. 735.

Lymnaea heterostropha Say, Nich. Encycl., 1817, pl. 1, fig. 6. "Delaware river."

ME. to Conn.- Common in most of the ponds and streams.

\section{*Physa ancillaria Say.}

Say, Journ. Acad. Nat. Sci. Phila., 1825, ser. 1, vol. 5, p. 124. "Delatware river near Easton, and *** Connecticut river above Hartford." Gould, Inv. Mass., 1870, p. 485, fig. 736.

Me.- Mud and Square Lakes, Aroostook Co.; Waterville; Prospect; North Warren.

N. H.- Merrimack River.

VT.-Lake Champlain; Hartland.

Mass.-Fresh Pond, Cambridge; Lynnfield.

Conn.- Connecticut River, above Hartford; near New Haven.

\section{*Physa sayii Tappan.}

Tappan, Amer. Journ. Sci., 1839, ser. 1, vol. 35, p. 269, pl. 3, fig. 3. "Lake Pipin, *** Portage County, Ohio."

Me.-Caribou (Nylander); Fort Kent.

\section{Physa fragilis Mighels.}

Mighels, Proc. Boston Soc. Nat. Hist., 1843 (?), vol. 1, p. 49. “Monmouth, Me." Binney, Smithson. Misc. Coll., 1865, vol. 7, art. 2, p. 89, fig. 153.

Me.-Monmouth, in a mill pond (Mighels).

Physa gyrina Say.

Say, Journ. Acad. Nat. Sci. Phila., 1821, ser. 1, vol. 2, p. 171. 
"Bowyer creek, near Council Bluff, [Iowa]." Binney, Smithson. Misc. Coll., 1865, vol. 7, art. 2, p. 77, fig. 130.

VT.-Lake Champlain (Adams).

?R. I.- Tiverton (Carpenter).

\section{Physa elliptica Lea.}

Lea, Trans. Amer. Phil. Soc., 1837, new ser., vol. 5, p. 115, pl. 19, fig. 83. No locality.

ME.-Aroostook Co.

*Aplexa hypnorum (Linné).

Dall, Harriman Alaska Exped., 1905, vol. 13, p. 106, fig. 81.

Bulla hypnorum Linné, Syst. Nat., ed. 10, 1758, vol. 1, p. 727. "in Europae Muscis."

Physa elongata Say, Journ. Acad. Nat. Sci. Phila., 1821, ser. 1, vol. 2, p. 171 .

Bulinus elongatus Gould, Inv. Mass., 1870, p. 486, fig. 738.

Me.- Portland; Bethel; Monmouth; Bangor; Woodland; North Warren; Saco.

N. H.- Keene.

VT.-Hartland; Burlington.

Mass.-Cambridge; Southborough; Milton.

R. I.- Tiverton.

Conn.- New Haven.

\section{LYMNAEIDAE.}

\section{*Lymnaea stagnalis appressa (Say).}

Baker, Spec. Publ. Chicago Acad. Sci., 1911, no. 3, p. 137, pl. 19, figs. 4-10; pl. 20, figs. 1-6; pl. 21, figs. 1-3.

Lymneus appressus Say, Journ. Acad. Nat. Sci. Phila., 1821, ser. 1, vol. 2, p. 168. "LAKe SUPERIor."

VT.-Lake Champlain; Burlington (Adams); mouth of the Winooski River (Dr. H. F. Perkins); Lake Hortonia; Chimney Point. 
*Pseudosuccinea columella (Say).

Baker, Spec. Publ. Chicago Acad. Sci., 1911, no. 3, p. 163, pl. 23, figs. 8-20; pl. 24, figs. 1-4.

Lymnaea columella Say, Journ. Acad. Nat. Sci. Phila., 1817, ser. 1, vol. 1, p. 14. No locality. [Cold Water Creek, Mo.]

Limnaea columella Gould, Inv. Mass., 1870, p. 471, fig. 723.

Limnea acuminata Adams, Amer. Journ. Sci., 1840, ser. 1, vol. 39 , p. 374.

ME.- York, Cumberland, Oxford, Knox and Penobscot Counties. $\mathrm{VT}$.

Mass.-New Bedford; Winchester; Danvers; Waltham.

R. I.- Tiverton.

ConN.- Branford.

\section{*Pseudosuccinea columella chalybea (Gould).}

Baker, Spec. Publ. Chicago Acad. Sci., 1911, no. 3, p. 171, pl. 24, figs. 5-13.

Lymnea chalybea Gould, Amer. Journ. Sci., 1840, ser. 1, vol. 38, p. 196. [CAMBridge, Mass.]

Limnaea columella var. chalybea, Gould, Inv. Mass., 1870, p. 472.

Me.-Thomaston; Union.

VT.- Lake Hortonia.

Mass.- Cambridge; Green Lodge Station, Neponset River; Duxbury; Uxbridge.

R. I.- Tiverton; Newport.

*Radix auricularia (Linné).

Baker, Spec. Publ. Chicago Acad. Sci., 1911, no. 3, p. 179, pl. 22, figs. 12-15. Clapp, Nautilus, 1913, vol. 26, p. 116.

Helix auricularia Linné, Syst. Nat., ed. 10, 1758, p. 774. "IN Europae fluVis, STAGNis."

Mass. - Common in the Charles River about three miles above the Dam, on both the Cambridge and the Boston shores. Introduced. 


\section{*Bulimnea megasoma (Say).}

Baker, Spec. Publ. Chicago Acad. Sci., 1911, no. 3, p. 184, pl. 25, figs. 1-6.

Lymneus megasomus Say, Narrative Long's Exped., 1824, vol. 2, p. 263, pl. 15, fig. 10. "Bois Blanc Lake, North-ivest TerriTORY."

Limnaea (Bulimnea) megasoma Binney, Smithson. Misc. Coll., 1865, vol. 7, art. 2, p. 37, figs. $46,47$.

VT.--Lake Champlain; Burlington (Adams); "Intervels," mouth of the Winooski River (H. F. Perkins).

?MASS.

*Acella haldemani (Deshayes).

Baker, Spec. Publ. Chicago Acad. Sci., 1911, no. 3, p. 192, pl. 18, fig. 1; pl. 26, figs. 1-4.

Lymnaea gracilis Jay, Cat. Shells, 1839, ed. 3, p. 112, pl. 1, figs. 10, 11 (non Zieten, 1830). "Essex Co., N. Y."

Limnaea (Acella) gracilis Binney, Smithson. Misc. Coll., 1865, vol. 7 , art. 2 , p. 69 , figs. 112,114 .

Limnaea haldemani Deshayes in Binney, Journ. de Conch., 1867, ser. 3, vol. 7, p. 428 (new name for L. gracilis Jay, preoccupied).

Vt.-Lake Champlain (Adams); mouth of Potash Brook (H. F. Perkins); Chimney Point.

\section{Galba caperata (Say).}

Baker, Spec. Publ. Chicago Acad. Sci., 1911, no. 3, p. 225, pl. 28, figs. 20-33; pl. 29, figs. 1-3.

Lymnaeus caperatus Say, New Harmony (Ind.) Disseminator, 1829, vol. 2, p. 230. [Indiana.]

Limnaea caperata Binney, Smithson. Misc. Coll., 1865, vol. 7, art. 2 , p. 56 , fig. 87 .

Me.- Ironbound Id.

Mass.- Westfield. 
R. I.- Smithfield.

Conn.- Housatonic.

*Galba umbilicata (C. B. Adams).

Baker, Spec. Publ. Chicago Acad. Sci., 1911, no. 3, p. 236, pl. 30, figs. $1-7$.

Limnea umbilicata C. B. Adams, Amer. Journ. Sci., 1840, ser. 1 , vol. 39, p. 374. "New Bedford, [Mass.]." Gould, Inv. Mass., 1870, p. 480 , fig. 731.

Me.- North Haven; Buckfield; Woodland; Fox Id., Penobscot River; Fort Kent.

N. H.- Keene.

VT. - Burlington.

MAss.-Cambridge; Salem; Cohasset; Southborough; Westfield; 'Duxbury.

R. I.- Pawtucket.

Conn.- Farmington River, Unionville.

Galba parva (Lea).

Baker, Spec. Publ. Chicago Acad. Sci., 1911, no. 3, p. 243, pl. 29, figs. 5-14; pl. 30, figs. 9-12.

Lymnea parva Lea, Proc. Amer. Phil. Soc., 1841, vol. 2, p. 33. "Cincinnati, Ohio."

ME.- Rockland.

Conn,

* Galba humilis modicella (Say).

Baker, Spec. Publ. Chicago Acad. Sci., 1911, no. 3, p. 259, pl. 19, figs. 31-37; pl. 31, figs. 1-9.

Lymneus modicellus Say, Journ. Acad. Nat. Sci. Phila., 1825, ser. 1, vol. 5, p. 122. "Owego, on the Susquehanna River near the state of New York."

Limnaea humilis Gould, Inv. Mass., 1870, p. 482, fig. 734 (non Say). 
Limnea linsleyi DeKay, Nat. Hist. N. Y., Moll., 1843, p. 72, pl. 4, fig. 74 .

Me.-Caribou; Woodland; Rockland.

N. H.

Mass.-Uxbridge; Duxbury; Pittsfield; Arlington.

Cons.

Galba humilis rustica (Lea).

Baker, Spec. Publ. Chicago Acad. Sci., 1911, no. 3, p. 268, pl. 31, figs. 10-14.

Lymnea rustica Lea, Proc. Amer. Phil. Soc., 1841, vol. 2, p. 33. "Poland, Ohio."

Me.-Saco.

Mass.-Woburn; Arlington; Cambridge (Clapp).

*Galba obrussa (Say).

Baker, Spec. Publ. Chicago Acad. Sci., 1911, no. 3, p. 270, pl. 26, figs. 8-13; pl. 31, figs. 20-37.

Lymneus obrussus Say, Journ. Acad. Nat. Sci. Phila., 1825, ser. 1, vol. 5, p. 123. "Harrowgate" [Philadelphia, Pa.].

Limnea desidiosa Haldeman, Monogr. Limniades, 1842, p. 31, pl. 10; p. 48, pl. 13, figs. 16-18.

Limnaea desidiosa Gould, Inv. Mass., 1870, p. 478, fig. 729 (non Say).

Me.-Westbrook; Saco; Aroostook Co.; Rockland.

VT.-Manchester.

Mass.-Waltham; Winchester; Williamstown; Neponset River.

R. I.- Olneyville.

Conn.- Near New Haven; Orange; Milford.

Galba obrussa peninsulae (Walker).

Baker, Spec. Publ. Chicago Acad. Sci., 1911, no. 3, p. 283, pl. 32, figs. 1-6. 
Lymnaea desidiosa var. peninsulae Walker, Nautilus, 1908, vol. 22, pp. 9, 16, pl. 2, fig. 7. " heAdWATERS of the UNion River, Ontonagon County, Michigan."

Me.-Unity (Berry); Thomaston (Lermond); Woodland.

Galba obrussa exigua (Lea).

Baker, Spec. Publ. Chicago Acad. Sci., 1911, no. 3, p. 285, pl. 32, figs. 7-14.

Lymnea exigua Lea, Proc. Amer. Phil. Soc., 1841, vol. 2, p. 33. "Tenn[Essee]."

ME.-Aroostook River, Caribou; Caribou Stream, Woodland (Nylander); Fort Kent; Orono.

Conn.- Branford.

\section{Galba obrussa decampi (Streng).}

Baker, Spec. Publ. Chicago Acad. Sci., 1911, no. 3, p. 289, pl. 32, figs. 15-22.

Limnaea desidiosa var. decampi Streng, Nautilus, 1896, vol. 9, p. 123, fig. "Brook's Lake, Newaygo Co., Mich."

ME.- Barren Brook, Caribou (Nylander).

*Galba palustris (Müller).

Baker, Spec. Publ. Chicago Acad. Sci., 1911, no. 3, p. 298, pl. 26, figs. 17-37; pl. 33, figs. 1-25; pl. 34, fig. 20.

Buccinum palustre Müller, Verm. Terr. et Fluv. Hist., 1774, vol. 2, p. 131. [Europe.]

Limnaea elodes Gould, Inv. Mass., 1870, p. 475, fig. 728.

Limnea expansa Haldeman, Monogr. Limniades, 1842, no. 4, p. 29, pl. 9, figs. 6-8.

ME.-Caribou; Scarboro.

VT.-Shelburn Bay and Larrabee Point, Lake Champlain; Hartland. 
Mass.-Cambridge; Chebacco Lake; Woburn.

R. I.- Providence and Worcester Canal near Long Bridge; Lonsdale.

ConN.- Housatonic River.

Galba elodes shurtleffii (Tryon).

Baker, Spec. Publ. Chicago Acad. Sci., 1911, no. 3, p. 330, pl. 34, fig. 13.

Lymnaca (Limnophysa) shurtleffi Tryon, Amer. Journ. Conch., 1866, vol. 2, p. 111, pl. 10, figs. 4, 5. "Weatogue, Hartford Co., Conn."

Conn.- Weatogue, Hartford Co.

*Galba reflexa (Say).

Baker, Spec. Publ. Chicago Acad. Sci., 1911, no. 3, p. 332, pl. 30, figs. 30, 31; pl. 35, figs. 3, 5-22; pl. 36, figs. 1-11; pl. 18, fig. 10.

Lymneus reflexus Say, Journ. Acad. Nat. Sci. Phila., 1821, ser. 1, vol. 2, p. 167. "Lakes Erie and Superior."

VT.-Lake Champlain (Roper).

*Galba pallida (C. B. Adams).

Baker, Spec. Publ. Chicago Acad. Sci., 1911, no. 3, p. 377, pl. 40, figs. $1-5$.

Limnea pallida C. B. Adams, Amer. Journ. Sci., 1840, ser. 1, vol. 39, p. 374. "Shoreham, VT."

Limnaea pallida Gould, Inv. Mass., 1870, p. 481, fig. 733.

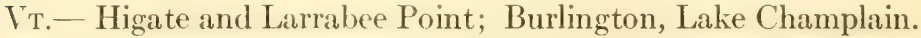
?MAss. - Andover (Adams).

\section{*Galba catascopium (Say).}

Baker, Spec. Publ. Chicago Acad. Sci., 1911, no. 3, p. 380, pl. 30, figs. 25, 26; pl.40, figs. $6-35$; pl. 61, figs. 1-4; pl. 47, fig. 10 . 
Lymnaea catascopium Say, Nich. Encycl., Amer. ed., 1817, vol. 2 (no pagination), pl. 2, fig. 3. "Delaware Rrver."

Lymneus pinguis Say, Journ. Acad. Nat. Sci. Phila., 1825, ser. 1, vol. 5, p. 123.

Lymnea linsleyi DeKay, Nat. Hist. N. Y., Moll., 1843, p. 72, pl. 4 , fig. 74 .

Me.-Waterville.

VT.-Burlington.

Mass.

Conn.-Stratford.

*Galba decollata (Mighels).

Baker, Spec. Publ. Chicago Acad. Sci., 1911, no. 3, p. 400, pl. 42, figs. 23-26; pl. 41, figs. 8-10.

Limnaea decollata Nighels, Proc. Boston Soc. Nat. Hist., 1843 (?), vol. 1, p. 49. "UNity, Me." Gould, Inv. Mass., 1870, p. 473, fig. 725 .

Me.- Winnecook Lake, Unity, Waldo Co.; Portland; Lincoln. ?Conn.- Housatonic River.

\section{*Galba emarginata (Say).}

Baker, Spec. Publ. Chicago Acad. Sci., 1911, no. 3, p. 408, pl. 30, figs. 27, 28; pl. 41, figs. 18-24; pl. 43, figs. 1-12.

Lymneus emarginatus Say, Journ. Acad. Nat. Sci. Phila., 1821, ser. 1, vol. 2, p. 170. "LAKes of Maine."

Limnaea emarginata Binney, Smithson. Misc. Coll., 1865, vol. 7, art. 2 , p. 51 , figs. 75,79 .

Me.- Mud Lake or Second Eagle Lake; west branch of Fish River between Eagle and Portage Lakes; St. John River, at Fort Kent.

\section{*Galba emarginata" mighelsii (Binney).}

Baker, Spec. Publ. Chicago Acad. Sci., 1911, no. 3, p. 416, pl. 43, figs. $13-21$; pl. 41 , fig. 25. 
Limnea ampla Mighels, Boston Journ. Nat. Hist., 1843, vol. 4, p. 347, pl. 16, figs. 1a, 1b, 1c (non Hartmann). "Second Eagle Lake, Maine."

Limnaea mighelsii Binney, Smithson. Misc. Coll., 1865, vol. 7, art. 2, p. 31, footnote.

Limnaea emarginata var. mighelsi Nylander, Distr. of Limnaea emarginata in Maine, 1901, pls. 3, 4.

Me.-Square Lake Inlet, Cross and Portage Lakes, Aroostook Co.; Sebago Lake.

*Galba oronoensis (Baker).

Lymnaea decollata oronoensis Baker, Nautilus, 1904, vol. 18, p. 62. "Orono, Maine."

Galba oronensis Baker, Spec. Publ. Chicago Acad. Sci., 1911, no. 3, p. 438, pl. 47, figs. 1-9.

Me.-Orono; Penobscot River at Lincoln; Caribou River, Aroostook Co.; Lake Auburn.

\section{*Planorbis antrosus Conrad.}

Conrad, Amer. Journ. Sci., 1834, ser. 1, vol. 25, p. 343. " RANdon's creek, near Clatborne, Alabama."

Planorbis bicarinatus Say, Nich. Encycl., 1817, vol. 2 (no pagination), pl. 1, fig. 4. Gould, Inv. Mass., 1870, p. 491, fig. 742. Walker, Nautilus, 1909 (non Lamarck, 1804), vol. 23, p. 1, pl. 1, fig. 3.

ME. to Conn.-Common in most of the ponds and streams.

\section{*Planorbis antrosus aroostookensis Pilsbry.}

Planorbis bicarinatus aroostookensis Pilsbry, Nautilus, 1895, vol. 8, p. 115. "East branch of Salmon brook, Woodland, Авоовтоок Co., Мє.” Walker, Nautilus, 1909, vol. 23, p. 7, pl. 1, figs. $1,2$.

Me.- East branch of Salmon Brook, Woodland; Collin's Pond; Caribou River, Caribou (Nylander). 
Planorbis antrosus striatus Baker.

Planorbis bicarinatus striatus Baker, Nautilus, 1902, vol. 15, p. 120. “Milwaukee, Wisconsin [pleistocene]." Baker, Trans. Acad. Sci. St. Louis, 1906, vol. 16, p. 9, pl. 1, fig. 11.

ME.- Square Lake; Rockville.

N. H.- Profile Lake.

\section{*Planorbis antrosus portagensis Baker.}

Planorbis bicarinatus portagensis Baker, Nautilus, 1908, vol. 22, p. 45. "Portage Lake, on Fish River, Aroostook County, Maine." Walker, Nautilus, 1909, vol. 23, p. 8, pl. 1, fig. 9.

ME.-Square Lake; Cross Lake; Second Lake and Moosehead Lake.

\section{*Planorbis trivolvis Say.}

Say, Nich. Encycl., 1817, vol. 2, pl. 11, fig. 2. "French Creek NEAR Lake Erie." Gould, Inv. Mass., 1870, p. 488, fig. 740.

ME. to ConN.- Common in most of the ponds and streams.

\section{*Planorbis trivolvis fallax Haldeman.}

Haldeman, Monogr. Limniades, 1844, no. 7, p. 15, pl. 3, figs. 1-3. "Massachusetts, Lake Erie, Indiana.?"

Planorbis lentus Gould, Inv. Mass., 1870, p. 490, fig. 741 (non Say).

ME.- Unity.

$\mathrm{V}$ T.

Mass.- Revere; Mystic Lake, Arlington.

\section{*Planorbis binneyi Tryon.}

Tryon, Amer. Journ. Conch., 1867, vol. 3, p. 197.

Planorbis corpulentus Gould, U. S. Expl. Exped. under Wilkes, 1852, vol. 12, p. 113; Atlas, pl. 8, figs. 130, a, b (non Say). "CouUmbia River, Oregon." 
Planorbis trivolvis Gould, Inv. Mass., 1870, p. 488 (in part), fig. 740 .

VT.-Lake Champlain.

Mass.-Dalton (Winkley).

\section{*Planorbis campanulatus Say.}

Say, Journ. Acad. Nat. Sci. Phila., 1821, ser. 1, vol. 2, p. 166. "CAyuga lake, [New York]." Gould, Inv. Mass., 1870, p. 492, fig. 743 .

Me. to Cons.-Common in most of the lakes and streams.

\section{*Planorbis (Menetus) exacuous Say.}

Planorbis exacuous Say, Journ. Acad. Nat. Sci. Phila., 1821, ser. 1, vol. 2, p. 165. "Lake Champlain."

Planorbis exacutus Gould, Inv. Mass., 1870, p. 495, fig. 746.

Me.- Norway.

VT.-Burlington; Chimney Point, Lake Champlain.

Mass._Lynnfield; Cambridge; Concord.

R. I.- Valley Falls Pond; near Pawtucket (Carpenter).

Conn.- Tashua Church Brook (Linsley); West River, near New Haven.

\section{*Planorbis (Gyraulus) hirsutus Gould.}

Planorbis hirsutus Gould, Amer. Journ. Sci., 1840, ser. 1, vol. 38, p. 196. [Mansfield, Mass.] Gould, Inv. Mass., 1870, p. 493, fig. 744 .

Planorbis albus Binney, Smithson. Misc. Coll., 1865, vol. 7, art. 2, p. 132, figs. 219-221 (non Müller).

Me.-Portage Lake; Rockland.

VT.

Mass.-Cambridge; Dedham; Dorchester; Sharon; Duxbury; Wareham.

R. I.-Providence and Worcester Canal; Valley Falls.

Conn.- Stonington; New Haven. 
*Planorbis (Gyraulus) deflectus Say.

Planorbis deflectus Say, Narrative Long's Exped., 1824, vol. 2, p. 261, pl. 15, fig. 8. "North-West Territory." Gould, Inv. Mass., 1870, p. 494, fig. 745 .

Planorbis virens Adams, Amer. Journ. Sci., 1840, ser. 1, vol. 39, p. 374.

Me.-Portage Lake.

VT.

Mass.- Winchester; Lynnfield; New Bedford; Cambridge.

R. I.- Valley Falls Pond.

Conn.- Bantam Lake, Litchfield; near New Haven.

*Planorbis (Gyraulus) dilatatus Gould.

Planorbis dilatatus Gould, Inv. Mass., 1841, p. 210, fig. 140. "Nantucket *** Hingham, [Mass.]." Gould, Inv. Mass., 1870, p. 498 , fig. 748 .

Me.- Harrison.

Mass.-Magnolia; Duxbury; Plymouth.

R. I.- Smithfield.

Conn.- Yantic River, Norwich: Stratford and near New Haven.

*Planorbis (Gyraulus) parvus Say.

Planorbis parvus Say, Nich. Encycl., 1817, vol. 2, pl. 1, fig. 5. "Delaware River." Gould, Inv. Mass., 1870, p. 497, fig. 747.

Planorbis elevatus C. B. Adams, Boston Journ. Nat. Hist., 1840, vol. 3, p. 327 , pl. 3, fig. 16 .

Me.-Barren Brook, Caribou; Portland; Rockland.

N. H.- Keene.

VT. - Burlington.

Mass.-Revere; Norfolk and Plymouth Counties; Pittsfield.

R. I.- Cunliff's Pond, Elmville.

*Planorbis (Armiger) crista (Linné).

Dall, Harriman Alaska Exped., 1905, vol. 13, p. 96. 
"Nautilus crista Linné, Syst. Nat., ed. 10, 1758, vol. 1, p. 709. "in Germaniae paludibus."

Planorbis nautileus Gray, Turton's Manual Land and Freshwater Shells, 1857, p. 236, pl. 8, fig. 94 .

Me.-Barren Brook, Caribou (Nylander).

*Segmentina armigera (Say).

Gould, Inv. Mass., 1870, p. 499, figs. 749, 750.

Planorbis armigerus Say, Journ. Acad. Nat. Sci. Phila., 1821, ser. 1, vol. 2, p. 164. "UPPER Missouri."

Me.- Bangor; Monmouth; North Warren.

Vт.-Burlington; Chimney Point.

Mass.-Auburndale; Cohasset; Revere; Andover.

R. I.- Tiverton.

Segmentina jenksii (H. F. Carpenter).

Davis, The Apteryx, 1905, vol. 1, p. 117, pl. 9, figs. A. B.

Planorbis jenksii Carpenter, Central Falls Visitor, March 2, 1871; Conch. Exch., 1887, vol. 2, p. 2. “NeAR Hammond's Pond, Pawtucket, R. I."

R. I.- Near Hammond's Pond, Pawtucket.

\section{ANCYLIDAE.}

*Ancylus fuscus C. B. Adams.

Adams, Boston Journ. Nat. Hist., 1840, vol..3, p. 329, pl. 3, fig. 17. "Andover *** Mansfield, [MAss.]." Walker, Nautilus, 1903, vol. 17, p. 15, pl. 1, figs. 1-9.

Mass. - Fresh Pond, Cambridge; Winchester.

Conn.- Near New Haven.

*Ancylus rivularis Say.

Say, Journ. Acad. Nat. Sci. Phila., 1817, ser. 1, vol. 1, p. 125. [United States.] Walker, Nautilus, 1904, vol. 18, pp. 16, 25, pl. 1, figs. 1-10, 13-15. 
ME.-Collins' Millpond, Caribou (Nylander).

Mass.

\section{*Ancylus tardus Say.}

Say, New Harmony (Ind.) Disseminator, Jan. 15, 1831. "WABASH River." Walker, Nautilus, 1904, vol. 18, p. 27, pl. 1, figs. 11, 12, 16-23.

ME.- Aroostook River, Caribou; Orono.

VT.- Hartland.

*Ancylus parallelus Haldeman.

Haldeman, Monogr. Limniades, 1841, no. 2, p. [3] of cover. "Vermont." Ibid., 1844, no. 7, Ancylus, p. 11, pl. 1, fig. 6. Walker, Nautilus, 1904, vol. 18, p. 77, pl. 5, figs. 1-9.

Ancylus rivularis Gould, Inv. Mass., 1841, p. 224, fig. 153 (non Say).

Me.-Collins' Millpond, Caribou Stream, Caribou; Cross Lake; Salmon Brook, Woodland (Nylander).

Ancylus ovalis Morse.

Morse, Journ. Portland Soc. Nat. Hist., 1864, vol. 1, p. 44, figs. 101, 102. “ "Bethel, ME.” Walker, Nautilus, 1904, vol. 18, p. 79.

ME.- Androscoggin River, at Bethel.

\section{Ancylus borealis Morse.}

Morse, Journ. Portland Soc. Nat. Hist., 1864, vol. 1, p. 45, figs. 103, 104. “PAtten, [ME.]." Walker, Nautilus, 1904, vol. 18, p. 80 , pl. 6 , figs. $14-16$.

ME.- Patten (Morse); St. John River, at Fort Kent (Nylander).

\section{Ancylus novangliae Walker.}

Ancylus (Ferrissia) novangliae Walker, Nautilus, 1908, vol. 21, p. 138, pl. 9. figs. 5-7. "Cambridge, Mass.

MAss. - A small pond near Cambridge. 


\section{Ancylus pumilus Sterki.}

Sterki, Eighth Ann. Rept. Ohio State Acad. Sci., 1900, p. 36. [Tuscarawas County, Ohio.] Walker, Nautilus, 1904, vol. 18, p. 82 , pl. 6 , figs. $20-22$.

ME.-Orono.

STYLOMMATOPHORA.

HELICIDAE.

*Helix (Tachea) hortensis Müller.

Helix hortensis Müller, Verm. Terr. et Fluv. Hist., 1774, vol. 2, p. 52. [Europe.] Gould, Inv. Mass., 1870, p. 429, fig. 688.

Helix subglobosa Binney, Boston Journ. Nat. Hist., 1867, vol. 1, p. 485 , pl. 17.

Tachea hortensis Morse, Journ. Portland Soc. Nat. Hist., 1864, vol. 1, p. 10, fig. 11 .

Me.-Cape Porpoise; Brown Cow, Inner Green and Cliff Islands, Casco Bay; Pumpkin Knob; Seal Rock; Little Egg Rock; Spruce Head; Bar Harbor; Little Duck Island; Isle au Haute.

Mass-- Rockport; Gloucester; Magnolia; Manchester; Salt, Eagle, House, and Kettle Islands; Cohasset; Provincetown; Chatham; Nantucket; Marthas Vineyard; Tuckernuck and Chappaquiddic Islands.

?CONN.-Weston (Linsley).

Note.-Helix aspersa Müller was recorded by Binney from Portland, Me., in 1869. It was also introduced at Woods Hole, Mass., in 1883 (Bull. U. S. Fish Comm., 1884, vol. 4, p. 87). There is now no evidence of its existence at either locality.

*Hygromia hispida (Linné).

Pilsbry, Manual Conch., 1894, ser. 2, vol. 9, p. 274.

Helix hispida Linné, Syst. Nat., ed. 10, 1758, vol. 1, p. 771. "Svecha."

Fruticicola hispida Binney, Bull. U. S. Nat. Mus., 1885, no. 28, p. 464 , fig. 505 . 
ME.-Rockland (Lermond).

Mass.- Marthas Vineyard, near Gay Head (Thomson).

\section{Hygromia rufescens (Pennant).}

Pilsbry, Manual Conch., 1894, ser. 2, vol. 9, p. 274.

Helix rufescens Pennant, Brit. Zool., 1777, vol. 4, p. 134, pl. 85, fig. 127. [ENGLAND.]

Fruticicola rufescens Binney, Bull. U. S. Nat. Mus., 1855, no. 28, p. 464 , fig. 506 .

Mass.- Naushon Island, near the "French water place" (Thomson).

\section{*Polygyra tridentata (Say).}

Pilsbry, Manual Conch., 1894, ser. 2, vol. 9, p. 76.

Helix tridentata Say, Nich. Encycl., 1816, vol. 2, pl. 2, fig. 1.

“Mrddle States." Gould, Inv. Mass., 1870, p. 422, fig. 680.

Triodopsis tridentata Binney, Bull. U.S. Nat. Mus., 1885, no. 28, p. 291, fig. 312.

N. H.-Concord; Squam Lake.

VT.-Burlington; St. Johnsbury; Newport; Coventry; Mt. Equinox; Sudbury; Mt. Ascutney.

Mass. - North Adams; Williamstown; Bashbish Falls; Chester.

Conn.-Stratford; Greenwich.

*Polygyra sayana Pilsbry.

Pilsbry, Proc. Acad. Nat. Sci. Phila., 1906, p. 127.

Helix diodonta Say, Narrative Long's Exped., 1824, vol. 2, p. 257, pl. 15, fig. 4 (non Muhlfeld). "NEW York."

Helix sayii A. Binney, Boston Journ. Nat. Hist., 1840, vol. 3, p. 379, pl. 16 (non Wood, 1828).

Me.-Caribou; Woodland; Capens, Moosehead Lake; Bethel; Bangor; Bridgeton. 
N. H.

Vt.-St. Johnsbury; Mt. Equinox; Mt. Ascutney.

Mass.-Westport; Williamstown; North Adams.

R. I.- Tiverton (Thomson).

\section{*Polygyra albolabris (Say).}

Pilsbry, Manual Conch., 1894, ser. 2, vol. 9, p. 76.

Helix albolabris Say, Nich. Encycl., 1816, vol. 2, pl. 1, fig. 1. [United States.]

Mesodon albolabris Binney, Bull. U. S. Nat. Mus., 1885, no. 28, p. 298, fig. 319 .

Me. to Conn.- Common in the woods and on many of the islands along the coast.

Polygyra albolabris dentata (Tryon).

Mesodon albolabris dentata Tryon, Amer. Journ. Conch., 1867, vol. 3 , p. 39 , pl. 7 , fig. 6 . "Maine, Massachusetts, Pennsylvania, Ohio, Iowa, Illinois, Michigan, \&C."

Mass.

R. I.- Tiverton.

*Polygyra dentifera (A. Binney).

Pilsbry, Manual Conch., 1894, ser. 2, vol. 9, p. 77.

Helix dentifera A. Binney, Boston Journ. Nat. Hist., 1837, vol. 1, p. 494, pl. 21. "Vermont."

Mesodon dentiferus Binney, Bull. U. S. Nat. Mus., 1885, no. 28, p. 312, fig. 333 .

Me.-Caribou; Woodland; Capens, Moosehead Lake; Bethel. N. H.- Fitzwilliam.

Vt. - East side of the Green Mts. (Adams); Mt. Ascutney. Mass.-Chester; North Adams. 
*Polygyra thyroidus (Say).

Pilsbry, Manual Conch., 1894, ser. 2, vol. 9, p. 77.

Helix thyroidus Say, Journ. Acad. Nat. Sci. Phila., 1817, ser. 1, vol. 1, p. 123. [United StATEs.]

Mesodon thyroides Binney, Bull. U. S. Nat. Mus., 1885, no. 28, p. 313 , fig. 335 .

ME.

IT.

Mass.-Cohasset; New Bedford; Westport; Dartmouth; Swansea; Williamstown; Blue Hill; Naushon Id.; Clarendon Hill.

R. I.- Tiverton; Cumberland.

Conn.-Greenwich.

\section{*Polygyra palliata (Say).}

Pilsbry, Manual Conch., 1894, ser. 2, vol. 9, p. 77.

Helix palliata Say, Journ. Acad. Nat. Sci. Phila., 1821, ser. 1, rol. 2, p. 152. "Illivois."

Triodopsis palliata Binney, Bull. U. S. Nat. Mus., 1885, no. 28, p. 284, fig. 303 .

VT.-Mt. Ascutney, Windsor Co.; Orange Co.

Mass. - Berkshire Co.

*Polygyra hirsuta (Say).

Pilsbry, Manual Conch., 1894, ser. 2, vol. 9, p. 78.

Helix hirsuta Say, Journ. Acad. Nat. Sci. Phila., 1817, ser. 1, vol. 1, p. 17. [United States.]

Stenotrema hirsutum Binney, Bull. U. S. Nat. Mus., 1885, no. 28, p. 278 , fig. 295 .

MAss.- Pittsfield (Roper).

Conn.-Stonington; Weston; New Haven; Greenwich.

*Polygyra fraterna (Say).

Walker, Rept. Geol. Surv. Mich., 1906, p. 470, fig. 23. 
Helix fraterna Say, Narrative Long's Exped., 1824, vol. 2, p. 25ૉ, pl. 15, fig. 3. "Pennsylvania."

Helix monodon Gould, Inv. Mass., 1870, p. 418, fig. 678.

Stenotrema monodon Binney, Bull. U. S. Nat. Mus., 1885, no. 28, p. 280, fig. 298 .

Me.-Caribou; Capens, Moosehead Lake; Bethel; Prospect; Ironbound Id.

N. H.-Livermore; Glen House; Jackson.

VT.-St. Johnsbury; Hartford; Burlington; Mt. Equinox; Mt. Ascutney.

Mass.-Williamstown; North Adams; Westport; Westfield.

R. I.- Tiverton.

Conn.-Orange; Stratford; New Haven; Greenwich.

CIRCINARIIDAE.

\section{* Circinaria concava (Say).}

Walker, Rept. Geol. Surv. Mich., 1906, p. 472, figs. 25-28.

Helix concava Say, Journ. Acad. Nat. Sci. Phila., 1821, ser. 1, vol. 2, p. 159. "Illuinors and Mrssouri."

Macrocyclis concava Gould, Inv. Mass. 1870, p. 406, fig. 668.

Me.-Bethel; Oxford Co.

VT.-St. Johnsbury; Cloak Island, Lake Champlain; Manchester; Newport.

Mass.- North Adams.

\section{ZONITIDAE.}

*Omphalina fuliginosa (Griffith).

Walker, Rept. Geol. Surv. Mich., 1906, p. 474, figs. 29-32.

Helix fuliginosa Griffith in Gould, Boston Journ. Nat. Hist., 1840, vol. 3, p. 417, pl. 24. "Soutiern, Middle, And Western STATEs."

Zonites fuliginosa Gould, Inv, Mass., 1870, p. 454, fig. 714. 
VT.-Mt. Equinox.

Mass.- Bashbish Falls.

Conn.-Stratford; Greenwich.

*Omphalina inornata (Say).

Walker, Rept. Geol. Surv, Mich., 1906, p. 475, fig. 33.

Helix inornata Say, Journ. Acad. Nat. Sci. Phila., 1822, ser. 1, vol. 2, p. 371. "Pennsylvania."

Zonites inornata Gould, Inv. Mass., 1870, p. 453, fig. 712.

VT.-Middlebury (Adams):

Mass.- North Adams; Chester.

*Vitrina limpida Gould.

Gould, Agassiz's Lake Superior, 1850, p. 243. "CAPE GourGanne, [Lake Superior].” Gould, Inv. Mass., 1870, p. 394, fig. 653.

Vitrina pellucida Adams in Thompson's Hist. Vt., 1842, p. 162.

Me.-Caribou; Fort Kent; Presque Isle; Orono; Bethel; Mt. Desert; near Portland and on several islands in Casco Bay.

VT.

Mass.- Westport.

*Vitrea cellaria (Müller).

Walker, Rept. Geol. Surv. Mich., 1906, p. 477, fig. 41.

Helix cellaria Müller, Verm. Terr. et Fluv. Hist., 1774, vol. 2,

p. 28. "in cellis vinaris Havniae."

Hyalina cellaria Gould, Inv. Mass., 1870, p. 395, fig. 654.

A European species introduced by commerce.

Me.-Portland; Saco; Castine.

Mass.-Salem; Marblehead; Lynn; Boston; New Bedford; Newtonville.

R. I.-Providence; Pawtucket.

Cons.- Huntington. 


\section{*Vitrea hammonis (Ström).}

Baker, Bull. Chicago Acad. Sci., 1902, no. 3, pt. 2, p. 180, figs. $36(2), 37$.

Helix hammonis Ström, Trondhjem. Selsk. Skrift., 1765, vol. 3, p. 435, fig. 16. [NoRwaY.]

Helix radiatula Alder, Trans. Nat. Hist. Soc. Northumberland, Durham, Newcastle-upon-Tyne, 1831, vol. 1, p. 38.

Hyalina electrina Gould, Inv. Mass., 1870, p. 397, fig. 656.

ME. to Conn.-A common and widely distributed species.

\section{*Vitrea binneyana (Morse).}

Walker, Rept. Geol. Surv. Mich., 1906, p. 479, fig. 44.

Hyalina binneyana Morse, Journ. Portland Soc. Nat. Hist., 1864, vol. 1, p. 13, text-fig. 25 ; pl. 2, fig. 9 ; pl. 4, fig. 13 ; pl. 6 , fig. 26. [Marne.] Gould, Inv. Mass., 1870, p. 400, fig. 659.

Me.-Caribou; Woodland; Bethel; Orono; Ironbound Id.

N. H.- Three-Mile Island, Lake Winnipesaukee.

VT.-Burlington.

Mass.-Cambridge; Westport; Lexington.

R. I.

\section{*Vitrea wheatleyi (Bland).}

Walker, Rept. Geol. Surv. Mich., 1906, p. 478, fig. 43.

Zonites wheatleyi Bland, Ann. N. Y. Acad. Sci., 1882, vol. 2, p. 368, fig. 1. “Knoxville, Tennessee***Tiverton, Rhode ISLAND."

R. I.-Tiverton, " rare, found with H. ferrea" (Thomson).

*Vitrea indentata (Say).

Walker, Rept. Geol. Surv. Mich., 1906, p. 480, fig. 48.

Helix indentata Say, Journ. Acad. Nat. Sci. Phila., 1822, ser. 1, vol. 2, p. 372. "Harrigate, [Philadelphia]."

Hyalina indentata Gould, Inv. Mass., 1870, p. 398, fig. 657. 
ME.-Cape Elizabeth.

$V_{T}$.

MAss.-Revere; Brookline; Westport; Cohasset; North Adams; Duxbury; Magnolia.

R. I.- Tiverton.

Conn.- Plymouth.

\section{Vitrea rhoadsi Pilsbry.}

Pilsbry, Nautilus, 1899, vol. 12, p. 101. "New Jersey: White Pond, Warren County." Pilsbry, Nautilus, 1906, vol. 19, p. 109, fig. 1.

ME.- Buckfield; Kennebunkport; South Portland.

Conn.- West Granby, Hartford Co.

\section{* Vitrea multidentata (Binney).}

Walker, Rept. Geol. Surv. Mich., 1906, p. 481, fig. 50.

Helix multidentata A. Binney, Boston Journ. Nat. Hist., 1840, vol. 3, p. 425, pl. 22, fig. 5. "VERMONT, ON THE EASTERN SLOPES of the Green Mountains.”

Hyalina multidentata Gould, Inv. Mass., 1870, p. 404, fig. 666.

Me.-Cumberland and Oxford Cos.; Buxton; Norway.

VT.- Middlebury; Hartland.

Mass. - Westport.

R. I.- Tiverton (Thomson).

\section{*Striatura ferrea Morse.}

Morse, Journ. Portland Soc. Nat. Hist., 1864, vol. 1, p. 17, figs. 36-39. [MAINE.]

Hyalina ferrea Gould, Inv. Mass., 1870, p. 401, fig. 663.

ME.-Bethel; Orono; Caribou; Woodland; Ironbound Id.

Mass.-Waverly; Duxbury; Westfield.

R. I.- Tiverton (Thomson). 
*Striatura milium (Morse).

Morse, Journ. Portland Soc. Nat. Hist., 1864, vol. 1, p. 18, fig. 41. Helix milium Morse, Proc. Boston Soc. Nat. Hist., 1859, vol. 7, p. 28. "MaIne."

Hyalina milium Gould, Inv. Mass., 1870, p. 401, fig. 662.

Me.-Bethel; Augusta; Portland; Orono; Woodland; Mt. Desert; Saco.

N. H.- Deering.

Mass.-Cambridge; Lexington; Duxbury.

R. I.- Tiverton.

\section{*Euconulus fulvus (Müller).}

Walker, Rept. Geol. Surv. Mich., 1906, p. 482, fig. 53.

Helix fulva Müller, Verm. 'Terr. et Fluv. Hist., 1774, vol. 2, p. 56. [EUROPE.]

Conulus fulvus Pilsbry, Nautilus, 1899, vol. 12, p. 115.

ME.-Woodland; Buckfield; Moosehead; Mt. Desert; Westbrook; Kennebunkport.

Mass. - Cambridge; New Bedford; Westfield.

\section{*Euconulus chersinus (Say).}

Hetix chersina Say, Journ. Acad. Nat. Sci. Phila., 1821, ser. 1, vol. 2, p. 156. " "SEA Istands of Georgia."

Hyalina chersina Gould, Inv. Mass., 1870, p. 402, fig. 664.

Conulus chersina Morse, Journ. Portland Soc. Nat. Hist., 1864, vol. 1, p. 19, fig. 46 .

Eucomulus chersinus polygyratus Walker, Rept. Geol. Surv. Mich., 1906, p. 482, fig. 54 .

ME.-Fort Kent; Bucksfield; Kennebunkport.

VT.

Mass.

*Zonitoides nitida (Müller).

Walker, Rept. Geol. Surv. Mich., 1906, p. 4S3, fig. 57. 
Helix nitida Müller, Verm. Terr. et Fluv. Hist., 1774, vol. 2, p. 32. " in SCALA LAPIDEA horti Fridrichsbergensis."

Hyalina nitida Binney and Bland, Smithson. Misc. Coll., 1869, vol. 8 , art. 3, p. 31, figs. 35,63 .

Me.-Bar Harbor.

MASS - Dorchester.

R. I.-Tiverton.

*Zonitoides arborea (Say).

Walker, Rept. Geol. Surv. Mich.,1906, p. 484, fig. 58.

Helix arboreus Say, Nich. Encycl., 1817, pl. 4, fig. 4. [United States.]

Hyalina arborea Gould, Inv. Mass., 1870, p. 396, fig. 655.

ME. to Conn.-Common on decaying logs and stumps.

\section{Zonitoides (Pseudohyalina) minuscula (Binney).}

Walker, Rept. Geol. Surv. Mich., 1906, p. 485, fig. 60.

Helix minuscula Binney, Boston Journ. Nat. Hist., 1S40, vol. 3 , p. 435 , pl. 22, fig. 4 . "Онго."

Pseudohyalina minuscula Morse, Journ. Portland Soc. Nat. Hist., 1864, vol. 1, p. 16, fig. 34 .

Hyalina minuscula Gould, Inv. Mass., 1870, p. 399, fig. 658.

ME.-Vicinity of Portland; Kennebunk; Rockland; North Haven.

VT.

Mass.-Westport; Duxbury; Blackstone.

R. I.- Tiverton.

Conn.- Hartford; Whittemore; New Haven.

*Zonitoides (Pseudohyalina) exigua (Stimpson).

Walker, Rept. Geol. Surv. Mich., 1906, p. 485, fig. 61.

IIelix exigua Stimpson, Proc. Boston Soc. Nat. Hist., 1850, vol. 3, p. 175. "Massachusetts, vicinity of Boston." 
Pseudohyalina exigua Morse, Journ. Portland Soc. Nat. Hist., 1S64, vol. 1, p. 16.

Hyalina exigua Gould, Inv. Mass., 1870, p. 400, figs. 660, 661.

Me.-Caribou; Woodland; Orono; Bethel; Mt. Desert.

Mass:-Cambridge; Salem; Westport; Duxbury; Wareham.

R. I.

Conn.- Stony Creek, New Haven.

\section{Gastrodonta suppressa (Say).}

Walker, Rept. Geol. Surv. Mich., 1906, p. 488, fig. 71.

IIelix suppressa Saty, New Harmony (Ind.) Disseminator, 1829, vol. 2, p. 229. "Pennsylvania."

Zonites suppressa Gould, Inv. Mass., 1870, p. 454, fig. 713.

Conn.

\section{LIMACIDAE.}

\section{*Limax maximus Linné.}

Linné, Syst. Nat., ed. 10, 1758, vol. 1, p. 652. No locality. Gould, Inv. Mass., 1870, p. 407, fig. [669.]

Introduced and gradually spreading inland from the seaports.

Me.-Portland; Bar Harbor.

Mass.-Boston; Cambridge; Auburndale; Wellesley; Milton.

R. I.- Newport.

Conn.

\section{Limax flavus Linné.}

Linné, Syst. Nat., ed. 10, 1758, vol. 2, p. 652. No locality. Gould, Inv. Mass., 1870, p. 410, fig. 672.

Limax variegatus Binney, Terr. Moll., 1851, vol. 2, p. 34, pl. 65, fig. 1 .

Introduced and confined chiefly to the seaports.

ME.- Portland. 
Mass.-Boston; Cambridge; New Bedford.

Conn.- New Haven.

*Agriolimax agrestris (Linné).

Walker, Rept. Geol. Surv. Mich., 1906, p. 489, figs. 72-74.

Limax agrestris Linné, Syst. Nat., ed. 10, 1758, vol. 2, p. 652. No locality. Gould, Inv. Mass., 1870, p. 408, fig. 670.

Limax tunicata Gould, Inv. Mass., 1841, p. 3.

Introduced from Europe and widely distributed.

ME.- Portland; Mt. Desert.

MAss.-Boston; Cambridge; New Bedford; Cohasset; Duxbury.

Conn.- Northfield; New Haven.

\section{*Agriolimax laevis campestris (Binney).}

Taylor, Monogr. Land and Freshwater Moll. Brit. Isles, 1907, pt. 10, p. 132 , fig. 145 .

Limax campestris Binney, Proc. Boston Soc. Nat. Hist., 1842 (?), vol. 1, p. 52. "New England States, New York, Ohio, Missourı.” Gould, Inv. Mass., 1870, p. 409, fig. 671.

Me. to ConN.- Common under wood and stones.

\section{ARIONIDAE.}

*Arion hortensis Férussac.

Férussac, Tabl. Syst. des Anim. Moll., 1819, pt. 1, Hist. Nat. Pulmonés sans Opercule, p. 65, pl. 2, figs. 4, 5. " PARIs, [France]." Taylor, Monogr. Land and Freshwater Moll. Brit. Isles, 1907, pt. 11 , p. 210 , pl. 24.

Arion fuscus Gould, Inv. Mass., 1870, p. 451, fig. 711.

Introduced from Europe.

ME.- Portland.

Mass.-Ipswich; Boston; Cohasset; New Bedford; Chester; Arlington.

Conn.- New Haven. 
Arion subfuscus cinereofuscus (Draparnaud).

Taylor, Monogr. Land and Freshwater Moll. Brit. Isles, 1907, pt. 11, p. 193, pl. 24.

Limax subfuscus cinerco-fuscus Draparnaud, Hist. Nat. Moll. France, 1805, p. 125, pl. 9, fig. 8. [France.]

Introduced from Europe.

MAss.- Jamaica Plain, Boston (Cockerell, Nautilus, 1905, vol. 19, p. 84).

\section{PHILOMYCIDAE.}

*Philomycus carolinianus (Bosc).

Limax carolinianus Bose, Hist. Nat. des Vers, suites à Buffon, ed. Déterville, 1802, vol. 1, p. 80, pl. 3, fig. 1. “L’Amérique SEPTENTRIONALE."

Limax togata Gould, Inv. Mass., 1841, p. 3.

Tebennophorus carolinensis Gould, Inv. Mass., 1870, p. 457, fig. 716 .

Philomycus carolinensis Baker, Bull. Chicago Acad. Sci., 1902, no. 3 , pt. 2, p. 201, pl. 30, fig. 1 .

Me.-Capens, Moosehead Lake; Isle au Haute.

N. H.

$\mathrm{VT}$.

Mass.-Cohasset; Westport; Uxbridge; North Adams; Chester.

Conn.-Stratford.

Philomycus pennsylvanicus Pilsbry.

Pilsbry, Proc. Acad. Nat. Sci. Phila., 1894, p. 22. "Yonk Furnace, York Co., Pa.*** South Mountain, near Pa. and Md. State boundary."

N. H.-Mt. Monadnock (Dr. W. H. Dall).

Pallifera dorsalis (Binney).

Morse, Journ. Portland Soc. Nat. Hist., 1864, vol. 1, p. 8, fig. 5, pl. 3, fig. 6 . 
Philomycus dorsalis Binney, Proc. Boston Soc. Nat. Hist., 1842 (?), vol. 1, p. 52. "Massachusetts and Vermont."

Tebennophorus dorsalis Gould, Inv. Mass., 1870, p. 460, fig. 717.

ME.- Isle au Haute.

VT.

Mass.- Westport.

ENDODONTIDAE.

\section{*Pyramidula alternata (Say).}

Baker, Bull. Chicago Acad. Sci., 1902, no. 3, pt. 2, p. 206, pl. 28 , figs. 19, 21-24.

Helix alternata Say, Nich. Encycl., 1817, vol. 2, pl. 1, fig. 2.

"Middle States." Gould, Inv. Mass., 1870, p. 412, fig. 673.

Anguispira alternata Morse, Journ. Portland Soc. Nat. Hist., 1864, vol. 1, p. 11, text-fig. 15; pl. 4, fig. 16.

ME. to ConN.- Common in woods under decaying logs.

*Pyramidula cronkhitei anthonyi Pilsbry.

Pilsbry, Proc. Acad. Nat. Sci. Phila., 1906, p. 153.

Helix striatella Anthony, Boston Journ. Nat. Hist., 1840, vol. 3, p. 278, pl. 3, fig. 2 (non Rang, 1831). "Cincinnati, [Оніо]." Gould, Inv. Mass., 1870, p. 413, fig. 674.

Patula striatella Morse, Journ. Portland Soc. Nat. Hist., 1864, vol. 1, p. 21, text-fig. 48; pl. 2, fig. 6; pl. 8, fig. 49.

ME. to ConN.- Common under the bark of decaying logs.

*Pyramidula cronkhitei catskillensis Pilsbry.

Pyramidula striatella catslillensis Pilsbry, Nautilus, 1898, vol. 12, p. 86. "Tannersville Valley, Catskill Mountains, N. Y.; White Pond, Warren County, N. J."

Míss.-Mt. Greylock.

*Pyramidula (Planogyra) asteriscus (Morse).

Walker, Rept. Geol. Surv. Mich., 1906, p. 494, fig. 84. 
Helix asteriscus Morse, Proc. Boston Soc. Nat. Hist., 1857, vol. 6, p. 128. "Bethel, Me."

Planogyra asteriscus Morse, Journ. Portland Soc. Nat. Hist., 1864, vol. 1, p. 24, text-figs. 50-52; pl. 2, fig. 5; pl. 8, fig. 53.

Me.-Caribou; Woodland; Orono; Gorham.

MAss.-Salem; Westport.

\section{${ }^{*}$ Helicodiscus parallellus (Say).}

Walker, Rept. Geol. Surv. Mich., 1906, p. 495, fig. 88.

Helix lineata Say, Journ. Acad. Nat. Sci. Phila., 1817, ser. 1, vol. 1, p. 18 (non Olivier).

Planorbis parallellus (sic) Say, Journ. Acad. Nat. Sci. Phila., 1821, ser. 1, vol. 2, p. 164 . "Upper MissourI."

Helicodiscus lineata Morse, Journ. Portland Soc. Nat. Hist., 1864, vol. 1, p. 25, text-figs. 61 , 62 ; pl. 2, fig. 3 ; pl. 8, fig. 63.

Hyalina lineata Gould, Inv. Mass., 1870, p. 404, fig. 667.

Me.-Woodland; Bethel; Mt. Desert; Little Duck Id.

VT.-Newport; Burlington.

Mass.-Revere; Duxbury; Westfield; Blackstone; Magnolia; Cohasset; Brookline.

R. I.

Conn.- New Haven.

\section{*Punctum pygmaeum minutissimum (Lea).}

Helix minutissima Lea, Trans. Amer. Phil. Soc., 1844, new ser., vol. 9, p. 17. "Cincinnati [, Ohio]."

Punctum minutissimum Morse, Journ. Portland Soc. Nat. Hist., 1864, vol. 1, p. 27, text-figs. 69 , 70 ; pl. 8, fig. 71.

Hyalina minutissima Gould, Inv. Mass., 1870, p. 403, fig. 665.

Me.-Caribou; Woodland; Orono; Bethel; Ironbound Id.

Mass-Cambridge; Westport; Duxbury; Lexington; Westfield.

R. I.- Tiverton. 
*Sphyradium edentulum (Draparnaud).

Dall, Harriman Alaska Exped., 1905, vol. 13, p. 54, fig. 37.

Pupe edentula Draparnaud, Hist. Nat. MLoll. France, 1805, p. 59, pl. 3, figs. 28, 29. [France.]

Pupa simplex Gould, Boston Journ. Nat. Hist., 1840, vol. 3, p. 403 , pl. 3 , fig. 21.

Vertigo simplex Gould, Inv. Mass., 1870, p. 444, fig. 706.

Me.-Woodland; Capens, Moosehead Lake; Orono; MIt. Desert.

N. H.- Francestown.

Mass.- Cambridge; Westport; Lexington.

\section{SUCCINEIDAE.}

*Succinea retusa Lea.

Lea, Trans. Amer. Phil. Soc., 1837, new ser., rol. 5, p. 117, pl. 19, fig. S6. " "Ohio, near Cincinnati."

Succinea ovalis Gould, Inv. Mass., 1870, p. 445, fig. 707 (non Say, 1817).

ME. to Conn.- Common in moist places.

\section{* Succinea ovalis Say.}

Say, Journ. Acad. Nat. Sci. Phila., 1817, ser. 1, vol. 1, p. 15. [North AmericA.]

Succinea campestris Gould, Inv. Mass., 1841, p. 195, fig. 126 (non Say).

Succinea obliqua Say, Narrative Long's Exped., 1824, vol. 2, p. 260, pl. 15, fig. 7. Gould, Inv. Mass., 1870, p. 447, fig. 709.

ME. to ConN.

*Succinea ovalis totteniana Lea.

Succinea totteniana Lea, Proc. Amer. Phil. Soc., 1841, vol. 2, p. 32. " Newport, R. I.” Gould, Inv. Mass., 1870, p. 448, fig. 710.

ME.- Prospect; Ironbound Id.

N. H - Francestown. 
Mass.-Fresh Pond, Cambridge; Revere; Cohasset.

R. I.- Newport.

*Succinea avara Say.

Say, Narrative Long's Exped., 1824, vol. 2, p. 260, pl. 15, fig. 6. "North-ivest Territory." Gould, Inv. Mass., 1870, p. 446 , fig. 708 .

Me.-Square Lake; Mt. Desert; Ironbound Id; Monmouth.

Mass.- Wayland; Cambridge; Duxbury.

Conn.- New Haven.

PUPILLIDAE.

* Strobilops labyrinthica (Say).

Pilsbry, Proc. Acad. Nat. Sci. Phila., 1892, p. 404.

Helix labyrinthica Say, Journ. Acad. Nat. Sci. Phila., 1817, ser. 1, vol. 1, p. 124. [United States.]

Strobila labyrinthica Morse, Journ. Portland Soc. Nat. Hist., 1864, vol. 1, p. 26, text-figs. 64-67; pl. 2, fig. 12, a-b; pl. 8, fig. 68 .

Me.-Caribou; Woodland; Orono; Sebago Lake; Mt. Desert.

N. H.-Three Mile Island.

VT.

Mass.-Lynnfield; Dartmouth; Westport; Cambridge; Westfield.

Conn.-Stonington; Hartford; New Haven.

\section{*Strobilops virgo (Pilsbry).}

Walker, Rept. Geol. Surv. Mich., 1906, p. 506, fig. 120.

Strobila labyrinthica var. virgo Pilsbry, Nautilus, 1892, vol. 6, p. 94. "Sebec Lake, Piscataquis Co., Maine."

ME.-Sebec Lake (Winkley); Bethel (Roper); Capens.

$V_{\mathrm{T}}$ - - Burlington. 
Strobilops affinis Pilsbry.

Pilsbry, Nautilus, 1893, vol. 7, pp. 56, 57. “New York, Оніо, ETC."

ME.- Bethel.

\section{*Zoơgenites harpa (Say).}

Morse, Journ. Portland Soc. Nat. Hist., 1864, vol. 1, p. 32, pl. 1, figs. 1-3.

Helix harpa Say, Narrative Long's Exped., 1824, vol. 2, p. 256, pl. 15, fig. 1. "North-West Territory."

Pupa costulata Mighels, Proc. Boston Soc. Nat. Hist., 18t4, vol. 1, p. 157.

Acanthinula harpa Binney, Bull. U. S. Nat. Mus., 1S85, no. 28, p. 185, fig. 183.

ME.-Fort Kent; Bethel; Waterville; Mt. Desert; North Haven; Sebago Lake.

N. H.-Berlin Falls; Wolfeboro.

VT.

Mass.-Westport; High Pines, Duxbury Beach.

R. I.- Tiverton.

\section{*Pupoides marginatus (Say).}

Pilsbry and Vanatta, Proc. Acad. Nat. Sci. Phila., 1900, p. 586.

Cyclostoma marginata Say, Journ. Acad. Nat. Sci. Phila., 1821, ser. 1, vol. 2, p. 172. “UPPer Missouri.'

Pupa fallax Gould, Inv. Mass., 1870, p. 436, fig. 696 (non Say).

Mass.- New Bedford.

R. I.- Tiverton.

\section{Bifidaria armifera (Say).}

Walker, Rept. Geol. Surv. Mich., 1906, p. 510, fig. 132.

Pupa armifera Say, Journ. Acad. Nat. Sci. Phila., 1821, ser. 1, vol. 2, p. 162. "Upper Missouri." Gould, Inv. Mass., 1870, p. 437 , fig. 697 . 
VT.

Mass.-Boston.

R. I.- Tiverton.

Conn.- - New Haven.

\section{*Bifidaria contracta (Say).}

Walker, Rept. Geol. Surv. Mich., 1906, p. 510, fig. 133.

Pupa contracta Say, Journ. Acad. Nat. Sci. Phila., 1822, ser. 1, vol. 2, p. 374. " "Virginia." Gould, Inv. Mass., 1870, p. 438, fig. 698.

Me-- Bethel; Bangor.

VT.-Burlington.

Mass.-Westport; Swampscott.

Conn.- Housatonic Valley.

*Bifidaria pentodon (Say).

Vanatta and Pilsbry, Nautilus, 1906, vol. 19, p. 122, pls. 6, 7, figs. 1-41.

Vertigo pentodon Say, Journ. Acad. Nat. Sci. Phila., 1822, ser. 1, vol. 2, p. 376. " "Pennsylvania."

Pupa curvidens Gould, Inv. Mass., 1841, p. 189, fig. 120.

Pupa pentodon Gould, Inv. Mass., 1870, p. 434, figs. 693, 694.

Me.-Buckfield; Woodland; Bethel; Fairfield; Westbrook; Orono.

VT. - Burlington.

Mass.- Cohasset; High Pines, Duxbury; Woburn.

P. I.

Conn.- New Haven.

Bifidaria tappaniana (C. B. Adams).

Vanatta and Pilsbry, Nautilus, 1906, vol. 19, p. 126, pl. 7, figs. 42-53.

Pupa tappaniana Adams, in Thompson's Hist. Vt., 1842, p. 158. [VERMONT.] 
ME.- Buckfield.

$V_{T}$.- Middlebury.

MAss. - Duxbury.

Bifidaria corticaria (Say).

Walker, Rept. Geol. Surv. Mich., 1906, p. 511, fig. 134.

Odostomia corticaria Say, Nich. Encycl., 1817, vol. 4, pl. 4, fig. 5.

Pupa corticaria Gould, Inv. Mass., 1870, p. 439, fig. 700.

ME.- Bethel; Gorham.

Mass.- Naushon Id.; Woburn.

Conn.- Orange.

*Pupilla muscorum (Linné).

Walker, Rept. Geol. Surv. Mich., 1906, p. 513, figs. 137-140.

Turbo muscorum Linné, Syst. Nat., ed. 10, 1758, vol. 1, p. 767. "In Europae muscosis."

Pupa muscorum Gould, Inv. Mass., 1870, p. 433, fig. 691.

ME.-Near Fort Preble; Ironbound Id.; Mt. Desert; Rockland.

Mass.-Provincetown; Dartmouth; Westport; High Pines, Duxbury.

Pupilla muscorum unidentata (C. Pfeiffer).

Walker, Rept. Geol. Surv. Mich., 1906, p. 514, fig. 141.

Pupa unidentata C. Pfeiffer, Naturgesch. Deutsch. Land- u. Süsswasser-Moll., 1821, vol. 1, p. 58, pl. 3, figs. 19, 20. " $\mathrm{BEY}$ Cassel auf deit Kratzenberge, und auf dem Schlosse zu Felsberg, [Germany]."

Pupa badia Adams, Boston Journ. Nat. Hist., 1S40, vol. 3, p. 331, pl. 3, fig. 18.

ME.- Thomaston.

Vertigo modesta (Say).

Dall, Harriman Alaska Exped., 1905, vol. 13, p. 29.

Pupa modesta Say, Narrative Long's Exped., 1824, vol. 2, p. 259, p. 15, fig. 5. "North-West Territory." 
Pupa decora Gould, Proc. Boston Soc. Nat. Hist., 1847, vol. 2, p. 263.

Me. - Cape Elizabeth.

Mass.

Conn.- Stonington.

*Vertigo gouldii (Binney).

Gould, Inv. Mass., 1870, p. 440, fig. 701.

Pupa gouldii Binney, Proc. Boston Soc. Nat. Hist., 1843 (?), vol. 1, p. 105. "North-Eastern and Middle States."

Isthmia gouldii Morse, Journ. Portland Soc. Nat. Hist., 1864, vol. 1, p. 38, text-fig. 95; pl. 10, fig. 96 .

ME.-Westbrook; Bethel; Woodland.

N. H.

Mass.-Cambridge; Roxbury; Westport.

R. I.- Tiverton.

ConN.- Northfield.

Vertigo gouldii paradoxa Sterki.

Sterki, Nautilus, 1900, vol. 13, p. 103. "Woodland, [Maine]." ME.-Woodland, Aroostook Co. (Nylander).

\section{*Vertigo bollesiana (Morse).}

Gould, Inv. Mass., 1870, p. 442, fig. 703.

Isthmia bollesiana Morse, Ann. Lyc. Nat. Hist. N. Y., 1865, vol. 8, p. 209, figs. 4-6. "Maine*** New Hampshire; Massachusetts; New York; And Norfolk, VA."

Me. - Ironbound Id. (Blaney); Bethel.

N. H.- Francestown.

MASS.

*Vertigo ventricosa (Morse).

Gould, Inv. Mass., 1870, p. 443, fig. 705. 
Isthmia ventricosa Morse, Ann. Lyc. Nat. Hist. N. Y., 1865, vol. 8, p. 207, figs. 1-3. "Maine; Concord, N. H.; Mohawk and Greenwich, N. Y."

ME.-Woodland; Westbrook; Bethel; Ironbound Id.

N. H.- Concord (Morse).

Conn.- New Haven.

\section{Vertigo ventricosa elatior Sterki.}

Sterki, Land and Fresh Water Moll. New Philadelphia, Ohio, 1894, p. 5. [Oнıо.]

ME.- Ironbound Id. (Blaney); Woodland; North Warren.

\section{*Vertigo ovata Say.}

Say, Journ. Acåd. Nat. Sci. Phila., 1822, ser. 1, vol. 2, p. 375.

“Pennsylvania." Gould, Inv. Mass., 1870, p. 442, fig. 704.

ME.- Portland.

N. H.- Portsmouth.

VT.- Middlebury; Hartland.

Mass.-Boston; Lynn; Cohasset; Westport; Duxbury.

R. I.- Tiverton.

Conn.-Stonington; Deep River; New Haven.

\section{Vertigo tridentata Wolf.}

Wolf, Amer. Journ. Conch., 1870, vol. 5, p. 198, pl. 17, fig. 1. "Canton, Illinors." Walker, Rept. Geol. Surv. Mich., 1906, p. 518 , fig. 153 .

ME.

Vertigo (Vertilla) milium (Gould).

Dall, Harriman Alaska Exped., 1905, vol. 13, p. 33, fig. 24.

Pupa milium Gould, Boston Journ. Nat. Hist., 1840, vol. 3, p. 402, pl. 3, fig. 23. "Chelsea, [Mass.]." 
Me.

VT.

Mass.-Oak Island; Chelsea; Westport; High Pines, Duxbury .

*Vertigo (Isthmia) pygmaea (Draparnaud).

Dall, Harriman Alaska Exped., 1905, vol. 13, p. 33.

Pupa pygmaea Draparnaud, Tableau, 1801, p. 57; Hist. Nat. Moll. France, 1805, p. 60, pl. 3, figs. 30, 31. [France.]

ME.

Mass.-Brookline; Waltham; Waverly.

\section{Vertigo nylanderi Sterki.}

Sterki, Nautilus, 1909, vol. 22, p. 107. "Woodland, Aroostook County, Maine."

ME.- Woodland.

\section{COCHLICOPIDAE.}

*Cochlicopa lubrica (Müller).

Dall, Harriman Alaska Exped., 1905, vol. 13, p. 33.

Helix lubrica Müller, Verm. Terr. et Fluv. Hist., 1774, vol. 2, p. 104. [Europe.]

Zua lubricoidea Morse, Journ. Portland Soc. Nat. Hist., 1864, vol. 1 , p. 30 , text-figs. $79-81,83,84$; pl. 10 , fig. 82 .

Cionella subcylindrica Gould, Inv. Mass., 1870, p. 431, fig. 690.

Me. to Cons.-Common in woods under leaves and bark.

\section{VALLONIIDAE.}

*Vallonia pulchella (Müller).

Pilsbry, Manual Conch., 1892, ser. 2, vol. 8, p. 248, pl. 32, figs. $1-5$.

IIelix pulchella Müller, Verm. Terr. et Fluv. Hist., 1774, vol. 2, p. 30. [Europe.] 
Helix minuta Say, Journ. Acad. Nat. Sci. Phila., 1817, ser. 1, vol. 1, p. 123.

Me. - Mt. Desert.

Mass.- New Bedford.

*Vallonia excentrica Sterki.

Sterki, Proc. Acad. Nat. Sci. Phila., 1893, p. 252, pl. \&, figs. B, M. [Europe and eastern North America.] Pilsbry, Manual Conch., 1892, ser. 2, vol. 8, p. 249, pl. 32, figs. 6-9.

Me.-Caribou; Saco; Cape Elizabeth; Cliff Island.

Mass.-Cambridge; Duxbury; New Bedford.

*Vallonia costata (Müller).

Pilsbry, Manual Conch., 1892, ser. 2, vol. 8, p. 252, pl. 32, figs. 18-22, 27; pl. 33, fig. 54 .

Helix costata Müller, Verm. Terr. et Fluv. Hist., 1774, vol. 2, p. 31. "IN LOCo elevato Fridrichsdalensis."

Me.-Great Cranberry Island; Rockland; Aroostook Co. Mass. - Uxbridge; Cambridge. 


\section{CEPHALOPODA.}

DIBRANCHIA.

DECAPODA.

\section{TEUTHIDAE.}

\section{Lestoteuthis fabricii (Lichtenstein).}

Verrill, Trans. Conn. Acad., 1881, vol. 5, p. 390, pl. 45, figs. 1-2d; pl. 49, figs. 1-1f; pl. 55, figs. 1-1d.

Sepia loligo Fabricius, Fauna Groenlandica, 1780, p. 358 (non Linné).

Onychoteuthis fabricii Lichtenstein, Isis, 1818, vol. 19.

Chiloteuthis rapax Verrill, Trans. Conn. Acad., 1881, vol. 5, p. 293, pl. 49, figs. 1-1f.

Onychoteuthis banksii of authors, not of Leach.

Gulf of Maine. - "From the stomach of a cod off Seal Island" (Verrill).

Abralia megalops Verrill.

Verrill, Amer. Journ. Sci., 1882, ser. 3, vol. 24, p. 364. [off Marthas Vineyard.] Verrill, Trans. Conn. Acad., 1884, vol. 6, p. 143 , pl. 28 , fig. 2 .

MAss.-Off Marthas Vineyard, 173 fathoms.

\section{OMMASTREPHIDAE.}

Ommastrephes illecebrosa (Lesueur).

Verrill, Trans. Conn. Acad., 1880, vol. 5, p. 268, pl. 28; pl. 29, figs. 5, 5a; pl. 37, fig. 8 ; pl. 39 .

Loligo illecebrosa Lesueur, Journ. Acad. Nat. Sci. Phila., 1821, ser. 1, vol. 2, p. 95, pl. [10]. "SANDY BAY, [MAss.]."

Ommastrephes sagittatus Gould, Inv. Mass., 1870, p. 510, pl. 26, figs. 341-344 (not L. pavo); not pl. 25, fig. 339, which really represents a Loligo (Verrill). 
Me.-Casco Bay; Mt. Desert; Eastport.

Mass.-Gloucester; Salem; Provincetown; Vineyard Sound. R. I.- Newport.

\section{Sthenoteuthis megaptera (Verrill).}

Verrill, Trans. Conn. Acad., 1880, vol. 5, p. 223, pl. 21, figs. 1-9. Architeuthis megaptera Verrill, Amer. Journ. Sci., 1878, ser. 3, vol. 16, p. 207. "Cape Sable, N. S."

Gulf of Maine._ "Cast ashore, during a severe gale, near Cape Sable" (Verrill); Georges Bank, from a codfish.

\section{CHIROTEUTHIDAE.}

Chiroteuthis lacertosa Verrill.

Verrill, Trans. Conn. Acad., 1881, vol. 5, p. 408, pl. 56, figs. 1-1f. "Brown's Bank, off Nova Scotia."

Chiroteuthis bonplandi (?) Verrill, Trans. Conn. Acad., 1881, vol. 5, p. 299 (non Verany).

Gulf of Maine--Brown's Bank, from the stomach of a cod (Verrill).

Brachioteuthis beanii Verrill.

Verrill, Trans. Conn. Acad., 1881, vol. 5, p. 406, pl. 55, figs. 33b; pl. 56, figs. 2-2a. " off Martha's Vineyard."

Mass.-Off Marthas Vineyard, in 183 to 368 fathoms; all from fish stomachs (Verrill).

\section{HISTIOTEUTHIDAE.}

Histioteuthis collinsii Verrill.

Verrill, Amer. Journ. Sci., 1879, ser. 3, vol. 17, p. 241. " ofF Nova Scotia." Verrill, Trans. Conn. Acad., 1880-81, vol. 5, pp. 234, 300, 404, pls. 22, 26; pl. 27, figs. 3-5; pl. 37, fig. 5 .

Georges Bank, northeastern part, in 180 fathoms. 


\section{DESMO'TEUTHIDAE.}

Taonius pavo (Lesueur).

Verrill, Trans. Conn. Acad., 1881, vol. 5, p. 306.

Loligo pavo Lesueur, Journ. Acad. Nat. Sci. Phila., 1821, ser. 1, vol. 2, p. 96, pl. [11]. "SANDy-BAy, [Mass.]."

Loligopsis pavo Binney in Gould, Inv. Mass., 1870, p. 509, not pl. 26, figs. 341-344 (Verrill). Tryon, Manual Conch., 1879, ser. 1, vol. 1, p. 163, pl. 68, fig. 252; pl. 69, fig. 253.

Mass.- Sandy Bay, Cape Ann.

\section{SPIRULIDAE.}

* Spirula spirula (Linné).

Hedley, Bull. New Zealand Colonial Mus., 1906, no. 1, p. 48.

Nautilus spirula Linné, Syst. Nat., ed. 10, 1758, vol. 1, p. 710. "America."

Spirula fragilis Gould, Inv. Mass., 1870, p. 516, fig. 755.

Spirula peronii Tryon, Manual Conch., 1879, ser. 1, vol. 1, p. 205, pl. 96, figs. 467-469; pl. 105, fig. 585 .

MAss - Nantucket; Marthas Vineyard; Muskeget and No Man's Land, 1904 (Sumner). Shells are occasionally cast upon the outer beaches.

\section{LOLIGINIDAE.}

\section{*Loligo pealeii Lesueur.}

Lesueur, Journ. Acad. Nat. Sci. Phila., 1821, ser. 1, vol. 2, p. 92, pl. 8. "South Carolina?"

Loligo pealii Binney in Gould, Inv. Mass., 1870, p. 514, pl. 25, figs. 339,340 .

Loligo punctata DeKay, Nat. Hist. N. Y., Moll., 1843, p. 3, pl. 1, fig. 1 . 
Me.-Casco Bay.

MAss.- Vineyard Sound, 5 to 20 fathoms; Hyannis; Provincetown.

R. I.- Narragansett Bay; off Newport and Point Judith, 16 to 26 fathoms.

Conn.- New Haven; Noank, 3 to 8 fathoms.

\section{Loligo pealeii borealis Verrill.}

Verrill, Amer. Journ. Sci., 1880, ser. 3, vol. 19, p. 292. " ANinisQuam, Mass." Verrill, Trans. Conn. Acad., 1881, vol. 5, pp. 308, 316 , pl. 37 , fig. 2 ; pl. 41, fig. 1 .

Mass.-Gloucester; Salem; Provincetown; Massachusetts Bay, 47 fathoms.

Loligo pealeii pallida Verrill.

Verrill, Trans. Conn. Acad., 1881, vol. 5, pp. 308, 317, pl. 34, figs. 1-4; pl. 37, figs. 9-11; pl. 40, fig. 1.

Loligo pallida Verrill, Rept. Inv. Vineyard Sound, 1873, p. 635, pl. 20, figs. 101, 101a. "Long Island Sound."

MAss. - Vineyard Sound, 5 to 16 fathoms; Nantucket Sound, 8 to 12 fathoms.

R. I.-Narragansett Bay, 6 to 12 fathoms; off Newport, $4 \frac{1}{2}$ to 16 fathoms.

ConN.- Fisher's Island Sound; off New Haven.

\section{SEPIOLIDAE.}

Stoloteuthis leucoptera (Verrill).

Verrill, Trans. Conn. Acad., 1881, vol. 5, p. 418.

Sepiola leucoptera Verrill, Amer. Journ. Sci., 1878, ser. 3, vol. 16, p. 378. "Gulf of Maine 30 miles E. from Cape Ann." Verrill, Trans. Conn. Acad., 1881, vol. 5, p. 347, pl. 31, figs. 4, 5; pl. 54, fig. 4.

Gulf of Maine, 30 miles east of Cape Ann, 110 fathoms. 
Mass.- Off Cape Cod, 94 to 122 fathoms; off Marthas Vineyard, 182 to 388 fathoms.

Rossia hyatti Verrill.

Verrill, Amer. Journ. Sci., 1878, ser. 3, vol. 16, p. 208. "MAssAChusetts Bay; *** off Cape Sable; *** off Halifax, [N. S.]." Verrill, 'Trans. Conn. Acad., 1881, vol. 5, p. 351, pl. 27, figs. 8, 9; pl. 30, fig. 1; pl. 31, figs. 1, 2; pl. 46, fig. 5.

Mass.-Off Cape Ann, 6 to 14 miles, in 45 to 90 fathoms; off Gloucester $4 \frac{1}{2}$ to 8 miles, in 42 to 45 fathoms; off Cape Cod, $6 \frac{1}{2}$ to 15 miles, in 45 to 80 fathoms.

Rossia sublaevis Verrill.

Verrill, Amer. Journ. Sci., 1878, ser. 3, vol. 16, p. 209. "MAssACHusetTS BAY."

Rossia sublevis Verrill, Trans. Conn. Acad., 1881, vol. 5, p. 354, pl. 30, fig. 2 ; pl. 31 , fig. 3 ; pl. 46, fig. 4 ; pl. 47, figs. $2-4$.

Mass.-Off Cape Ann, 6 to 33 miles, in 54 to 110 fathoms; off Cape Cód, 11 to 15 miles, in 45 to 80 fathoms.

R. I.- Off Newport, 155 to 372 fathoms.

Heteroteuthis tenera Verrill.

Verrill, Amer. Journ. Sci., 1880, ser. 3, vol. 20, p. 392. [oFf Marthas Vineyard.] Verrill, Trans. Conn. Acad., 1881, vol. 5, pp. $357,419$.

Mass.-Off Marthas Vineyard, 64 to 192 fathoms. Most abundant in 125 to 150 fathoms.

R. I.- Off Newport, 85 to 252 fathoms.

OCTOPODA.

\section{PHILONEXIDAE.}

Parasira catenulata (Férussac).

Verrill, Trans. Conn. Acad., 1881, vol. 5, p. 362, pl. 33, figs. 2, 2 a.

Octopus catenulatus Férussac, Poulpes, 1828, pls. 6, 6 bis, 6 ter. 
Octopus granulatus Verrill, Amer. Journ. Sci., 1878, ser. 3, vol. 16, p. 210 (non Lamarck).

Mass.- Vineyard Sound.

\section{ARGONAUTIDAE.}

\section{Argonauta argo americana Dall.}

Dall, Bull. U. S. Nat. Mus., 1889, no. 37, pp. 174, 200, pl. 43, figs. 1a-b; pl. 64, fig. 142b; pl. 67, figs. 1-3. [SOUTHEASTERN COAST United STATES.]

Argonauta argo Verrill, Trans. Conn. Acad., 1881, vol. 5, pp. 364, 420; Ibid., 1884, vol. 6, p. 247, pl. 28, figs. 1-1b.

Mass.-Off Marthas Vineyard, 64 to 365 fathoms.

\section{POLYPODIDAE.}

\section{*Polypus arcticus (Prosch).}

Hoyle, Journ. Conch., 1902, vol. 10, pp. 197, 204.

Octopus arcticus Prosch, Kong. Dansk. Vid. Selsk. Skrift., 1819, ser. 5, nat. math. afd., vol. 1, p. 59. "GRöNLAND."

Octopus bairdii Verrill, Amer. Journ. Sci., 1873, ser. 3, vol. 5, p. 5; Trans. Conn. Acad., 1881, vol. 5, p. 368, pl. 33, figs. 1, 1a; pl. 34, figs. 5, 6; pl. 36, fig. 10; pl. 38, fig. 8; pl. 49, figs. 4, 4a; pl. 51, figs. 1, 1a.

ME.-Off Eastport, 75 to 80 fathoms; off Casco Bay, 50 to 95 fathoms (Verrill).

Mass.-Off Cape Ann, 5 to 13 miles, in 42 to 75 fathoms; off Gloucester, $4 \frac{1}{2}$ miles, in 43 to 45 fathoms; off Cape Cod, 14 to 15 miles, in 70 to 94 fathoms; off Marthas Vineyard, 120 to 410 fathoms.

R. I.- Off Newport, 85 to 192 fathoms (Verrill). 



\section{INDEX TO GENERA AND SUBGENERA.}

Numbers in heary-face type indicate the page on which the genus or subgenus first occurs as a valid name in the list. Page numbers in plain type refer to synonyms. If a name occurs on two or more consecutive pages, the number of the first page only is given.

Abra, 75 .

Abralia, 218.

Acanthinula, 211.

Acanthodoris, 173

Acella, 182.

Acirsa, 104

Aclis, 93, 105

Acmaea, 85.

Acrybia, 107.

Actaeon, 147.

Acteon, 99, 147.

Actinobolus, 61.

Adalaria, 173.

Adeorbis, 87, 120.

Admete, 140.

Aeolidia, 162.

Aeolis, 162.

Aequipecten, 28.

Agriolimax, 205.

Agriopoma, 69.

Alasmidonta, 26.

Alasmodonta, 23, 26.

Alderia, 161.

Alectrion, 110, 134.

Alexia, 178.

Aligena, 67.

Amauropsis, 107.

Amicula, 10.

Amnicola, 114.

Amphidesma, 75.

Amphisphyra, 149.

Amphitrite, 169.

Ampullina, 107.

Anachis, 132.
Anatina, 36.

Ancula, 176.

Ancylus, 192.

Anguispira, 207.

Angulus, 72.

Anodon, 23.

Anodonta, 24.

Anodontoides, 25.

Anomia, 31, 156.

Aphrodite, 69.

Aplexa, 180.

Aplysia, 153.

Aporema, 35.

Aporrhais, 128.

Arca, 12, 13, 18.

Architectonica, 120.

Architeuthis, 219.

Arctoscala, 103.

Argina, 18.

Argonauta, 223.

Arion, 205.

Armiger, 191.

Arrhoges, 128.

Assiminea, 111

Assiminia, 111.

Astarte, 42.

Astyris, 133.

Atalanta, 129.

Atlanta, 129, 155, 150.

Auricula, 178 .

Avicula, 20.

Axinopsis, 65.

Axinulus, 64.

Axinus, 65 . 
Balantium, 158.

Barnea, 79.

Bathyarca, 19, 20.

Bela, 141, 146.

Bifidaria, 211.

Bittium, 126, 127.

Boreoscala, 104.

Brachioteuthis, 219.

Brachydontes, 33.

Buccinum, 125, 131, 135, 138, 185.

Bulbus, 107.

Bulimnea, 182.

Bulinus, 180.

Bulla, 147, 180 .

Bullaria, 150.

Busycon, 139.

Bythinia, 115.

Cadlina, 171.

Cadulus, 84.

Caecum, 123.

Calliopaea, 160.

Calliostoma, 90.

Callocardia, 69.

Calyculina, 50.

Calyptraea, 109.

Campeloma, 111.

Camptonectes, 30.

Cancellaria, 140.

Capulus, 110.

Cardiomya, 41, 42.

Cardita, 60.

Cardium, 68.

Carinaria, 129.

Carychium, 177.

Cavolina, 156, 166.

Cemoria, 86.

Cerastoderma, 68.

Cerithiopsis, 126.

Cerithium, 126.

Ceronia, 77.

Cerophora, 129.

Chaetoderma, 10.

Chaetopleura, 8.
Chemnitzia, 93, 94, 100.

Chiloteuthis, 218.

Chiroteuthis, 219.

Chiton, 7.

Chlamys, 28.

Chrysallida, 97.

Cingula, 111, 116.

Cionella, 216.

Circinaria, 198.

Cirsotrema, 103.

Cithna, 120.

Clausina, 64.

Cleodora, 158 .

Clidophora, 37, 38 .

Clio, 155, 158.

Clione, 160.

Cocculina, 87.

Cochlicopa, 216.

Cochlodesma, 36.

Colombella, 132.

Columbella, 132.

Colus, 137.

Conulus, 202.

Corbula, 39, 78.

Corneocyclas, 58 .

Corolla, 159.

Coryphella, 165.

Couthouyella, 101.

Crassina, 44.

Crassinella, 45.

Cratena, 162.

Cremula, 101.

Crenella, 34.

Crepidula, 109.

Creseis, 157.

Crucibulum, 109.

Cryptodon, 62.

Cryptonatica, 105.

Cumingia, 74.

Cuspidaria, 40.

Cuthona, 162, 167.

Cuvieria, 159.

Cuvierina, 169.

Cyclas, 45. 
Cyclocardia, 60, 61 .

Cyclopecten, 30.

Cyclostoma, 112, 116, 211.

Cylichna, 148, 150.

Cymbulia, 159.

Cyprina, 42.

Cyrena, 71.

Cyrtodaria, 78.

Cytherea, 69.

Dacrydium, 40.

Defrancia, 141, 145.

Dendronotus, 169.

Dentalium, 82.

Diacria, 157.

Diaphana, 148, 149.

Diplodonta, 62.

Divaricella, 62.

Dolium, 128.

Doridella, 177.

Doris, 169, 171.

Doto, 169.

Drillia, 140.

Elysia, 161.

Elysiella, 161.

Embletonia, 164.

Ensatella, 75.

Ensis, 75.

Entalis, 82.

Eolidia, 164.

Eolis, 162.

Epitonium, 102.

Euconulus, 202.

Eudolium, 128.

Eulima, 91.

Eulimella, 92, 105.

Eumeta, 127.

Eupleura, 130.

Eurynea, 22.

Euspira, 106.

Evalea, 98.

Evalina, 98.
Facelina, 168.

Fasciolaria, 139.

Ferrissia, 193.

Fiona, 168.

Firola, 129.

Firoloida, 128.

Fossarus, 120.

Fruticicola, 194.

Fulgur, 139.

Fulvia, 69.

Fusus, 131, 136, 142, 144, 154.

Galba, 182.

Galvina, 165.

Gastranella, 72.

Gastrodonta, 204.

Geitodoris, 171.

Gemma, 70.

Glaucus, 168.

Glycimeris, 79 .

Glycymeris, 78 .

Goniobasis, 125.

Gouldia, 45.

Gymnobela, 146.

Gyraulus, 190.

Halicardia, 39.

Haminea, 151.

Hanleya, 7.

Helicodiscus, 208.

Helix, 4, 101, 108, 115, 181, 194, 207, $210,216$.

Helonyx, 84.

Hemimactra, 76.

Hermaea, 160.

Heterofusus, 154.

Heteroteuthis, 222.

Hima, 134.

Histioteuthis, 219.

Homalogyra, 119.

Hormomya, 32.

Hyalaea, 156.

Hyalina, 199, 208.

Hyalocyclis, 158. 
Hyalocylix, 158.

Hygromia, 194.

Ianthina, 102.

Idalia, 176.

Idaliella, 176.

Ilyanassa, 135.

Iolaea, 98.

Ischnochiton, 9.

Issa, 171.

Isthmia, 214, 216.

Jaminia, 92, 97.

Janthina, 101.

Kellia, 65, 66.

Kelliopsis, 68.

Kennerleyia, 37.

Kennerlia, 37.

Koonsia, 154.

Lacuna, 121.

Laevicardium, 68.

Lamellaria, 108.

Lamellidoris, 173.

Lampsilis, 21.

Leda, 13.

Lepeta, 86.

Lepetella, 86.

Lepidopleurus, $\mathbf{7}$.

Leptaxinus, 66.

Leptochiton, 7 .

Lepton, 66.

Lestoteuthis, 218.

Lima, 31 .

Limacina, 154.

Limaea, 31.

Limapontia, 161.

Limax, 162, 168, 175, 204.

Limnaea, 111, 181, 184.

Limnea, 181, 183, 188.

Limnophysa, 186.

Limopsis, 17.

Liocardium, 68.
Liocyma, 70.

Liostomia, 101.

Litiopa, 119.

Litorina, 120.

Littorina, 121.

Littorinella, 116.

Loligo, 218, 220.

Loligopsis, 220.

Lophyrus, 9.

Loripes, 61.

Lucina, 61.

Lucinoma, 61.

Lunatia, 106.

Lymnaea, 179, 180, 185.

Lymnaeus, 182.

Lymnea, 183, 187.

Lymneus, 180, 182, 186.

Lyonsia, 38.

Lyonsiella, 39.

Lyogyrus, 114.

Lyrodes, 82.

Lyrodus, 82.

Machaera, 75.

Machaeroplax, 89.

Macoma, 73.

Macrocyclis, 198.

Mactra, 74, 76.

Mamma, 106.

Mangilia, 145.

Margarita, 87.

Margaritana, 26, 28.

Margarites, 87.

Marginella, 140.

Marsenina, 108.

Martesia, 80.

Megayoldia, 15.

Melampus, 178.

Melanella, 91.

Melania, 125.

Melantho, 111.

Menestho, 98, 101.

Menetus, 190.

Mesodesma, 77. 
Mesodon, 196.

Metaptera, 23.

Microyoldia, 15.

Modiola, 33, 40.

Modiolaria, 33.

Modiolus, 33.

Molleria, 87.

Monoculus, 156.

Monodonta, 26.

Montacuta, 66, 67.

Montagua, 162.

Mulinia, 77.

Murex, 126, 131, 139.

Musculium, 48.

Musculus, 33.

Mya, 22, 27, 38, 41, 77.

Myrtaea, 61.

Mysella, 67.

Mytilimeria, 39 .

Mytillus, 33.

Mytilus, 32

Nassa, 133.

Natica, 105.

Nautilus, 192, 220.

Neaera, 40.

Neptunea, 136, 137.

Neptunella, 138.

Nerita, 107.

Neverita, 105.

Nicania, 45.

Niobe, 161

Noëtia, 18.

Nucella, 132.

Nucula, 11.

Octopus, 222.

Odostomia, 92, 97, 213.

Omalaxis, 120.

Ommastrephes, 218.

Omphalina, 4, 198.

Onchidoris, 174.

Onoba, 118.
Onychoteuthis, 218.

Opalia, 104.

Osteodesma, 38.

Ostrea, 20, 29.

Oxinoe, 109.

Oxygyrus, 130.

Paedoclione, 160.

Palio, 172.

Pallifera, 206.

Palliolum, 30.

Paludestrina, 115.

Paludina, 111, 114.

Pandora, 37.

Pandorina, 38.

Panomya, 79.

Panopaea, 79.

Parasira, 222.

Pasithea, 127.

Patella, 85, 109.

Patula, 207.

Pecchiolia, 39.

Pecten, 28.

Pectunculus, 17.

Peracle, 159.

Periploma, 36.

Petricola, 71.

Phacoides, 61.

Phasianella, 99.

Philine, 151.

Philomycus, 206.

Pholadomya, 35.

Pholas, 79.

Physa, 179.

Pisidium, 51.

Placobranchus, 161.

Placopecten, 29.

Placophora, 10.

Plagioctenium, 29.

Planogyra, 207.

Planorbis, 188, 208.

Plaxiphora, 10.

Pleurobranchaea, 154.

Pleurotoma, 140, 142, 144. 
Pleurotomella, 146.

Polinices, 105.

Polycera, 172.

Polycerella, 172.

Polygyra, 4, 195.

Polypus, 223.

Pomatiopsis, 116.

Poromya, 38.

Portlandia, 15.

Propeamusium, 31.

Proptera, 23.

Protocardia, 69.

Pseudamusium, 30.

Pseudohyalina, 203.

Pseudosuccinea, 181.

Pteria, 20.

Ptychatractus, 139.

Ptycheulimella, 93.

Pulsellum, 84.

Punctum, 208.

Puncturella, 86.

Pupa, 177, 209, 211.

Pupilla, 213.

Pupoides, 211.

Purpura, 132.

Pyramidella, 92, 98.

Pyramidula, 207.

Pyramis, 93, 101.

Pyrgiscus, 94.

Radix, 181.

Ranella, 130.

Retusa, 147.

Ringicula, 151.

Rissoa, 101, 116.

Rissoella, 99, 101.

Rochefortia, 66.

Rossia, 222.

Rostellaria, 128.

Saxicava, 78.

Scala, 103.

Scalaria, 102.

Scaphander, 149.
Scapharea, 18.

Scissurella, 87.

Scyllaea, 170.

Segmentina, 192.

Seila, 127.

Sepia, 218.

Sepiola, 221.

Serripes, 69.

Siliqua, 75.

Sipho, 137.

Siphodentalium, 83.

Skenea, 119.

Solariella, 89.

Solarium, 120.

Solecurtus, 74 .

Solemya, 11.

Solen, 74, 75.

Solenomya, 11.

Somatogyrus, 115.

Sphaerella, 62.

Sphaerium, 45, 49.

Sphyradium, 209.

Spirialis, 155.

Spirula, 220.

Spisula, 76.

Stenotrema, 197.

Sthenoteuthis, 219.

Stiliger, 160.

Stoloteuthis, 221.

Striatura, 201.

Strobila, 210.

Strobilops, 210.

Strophitus, 23.

Stylifer, 92.

Styliola, 157, 158.

Succinea, 209.

Sulcorinella, 98.

Sycotypus, 139.

Symphynota, 25.

Syrnola, 92.

Tachea, 194.

Tagelus, 74 .

Taonius, 220. 
Tapes, 70.

Tebennophorus, 206.

Tectura, 85.

Tellimya, 67.

Tellina, 52, 58, 72.

Teredo, 80.

Tergipes, 162, 164.

Tethys, 153.

Thais, 132.

Thracia, 36.

Thyasira, 62.

Tonicella, 8.

Torellia, 124.

Tornatella, 147.

Tornatina, 147.

Totteniana, 71.

Trachydermon, 9.

Trichotropis, 124.

Tridonta, 44.

Triforis, 126.

Trigonulina, 39.

Triodopsis, 195, 197.

Triphoris, 126.

Triptera, 159.

Tritia, 135

Tritonia, 169.

Tritonium, 131, 136, 142.

Trochus, 88.

Trophon, 131.

Tropidocyclas, 58.

Truncatella, 111, 119.

Turbo, 87, 89, 111, 116, 119, 213.

Turbonilla, 92, 93.

Turritella, 94, 98, 104, 117, 122, 123,127 .
Turritellopsis, 123.

Turtonia, 67.

Typhlomangelia, 145.

Unio, 21, 25, 27.

Urosalpinx, 132.

Utriculus, 147, 150, 152.

Vallonia, 216.

Valvata, 112.

Velutina, 108.

Venericardia, 60.

Venus, 42, 67, 70.

Bermetus, 123.

Vermicularia, 123.

Verticordia, 39.

Vertigo, 209, 212, 213.

Vertilla, 215.

Vitrea, 199.

Vitrina, 199.

Viviparus, 112.

Volvaria, 147, 150.

Xylophaga, 80.

Xylotrya, 82.

Yoldia, 14.

Yoldiella, 15.

Zirfaea, 80.

Zonites, 198, 204.

Zonitoides, 202.

Zoögenites, 211.

Zua, 216. 




a.

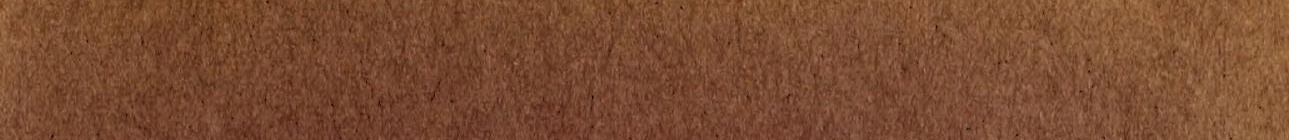
a (3)

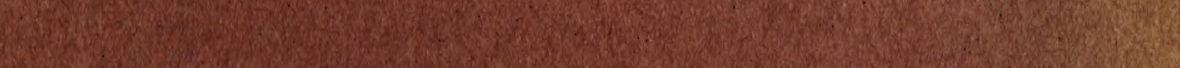
The

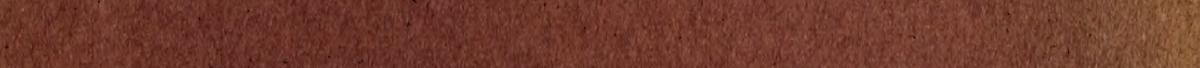
(3) (3)

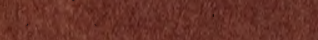

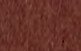
Wain
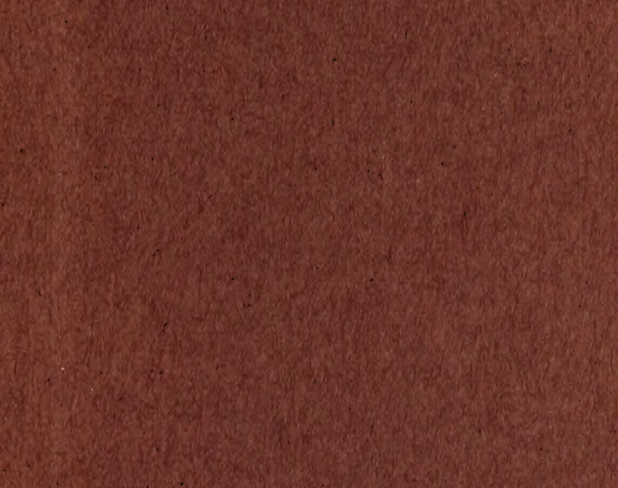

(20)

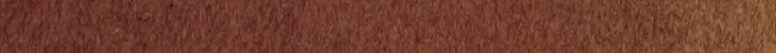
a.3.

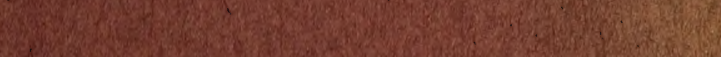
350. 1.

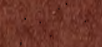

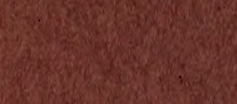
(1) 
